\title{
The Search for Low Energy Single Photons in MicroBooNE
}

\author{
A thesis submitted to The University of Manchester for the \\ degree of Doctor of Philosophy in the Faculty of Science and \\ Engineering
}

$\cdot 2018 \cdot$

Robert G. Murrells

School of Physics and Astronomy 


\section{Contents}

\begin{tabular}{lll}
\hline 1 & Introduction & 11
\end{tabular}

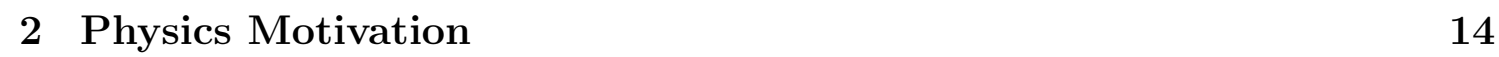

2.1 Overview . . . . . . . . . . . . . . . . . . . . . . . 14

2.1 .1 Discovery of Neutrino Flavour Transitions . . . . . . . . . . . 14

2.1 .2 Global Three Neutrino Oscillation Picture . . . . . . . . . . . 15

2.2 The MiniBooNE Low Energy Excess . . . . . . . . . . . . . . . . . . 17

2.3 Interpretations of the Low Energy Excess . . . . . . . . . . . . . . 20

$2.3 .1 \quad$ Neutral Current $\Delta$ Radiative Decay . . . . . . . . . . . . . . . 20

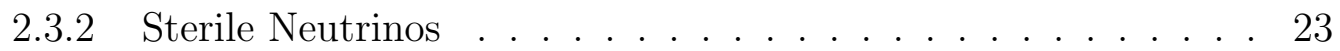

\begin{tabular}{|ll|}
\hline 3 & The MicroBooNE Experiment
\end{tabular}

3.1 Neutrino Flux at MicroBooNE . . . . . . . . . . . . . . . . . . . . . 25

3.2 Detector . . . . . . . . . . . . . . . . . . . . 30

3.2 .1 Liquid Argon Time Projection Chamber . . . . . . . . . . . . 30

$3.2 .2 \quad$ Operating Principles . . . . . . . . . . . . . . . . . 32

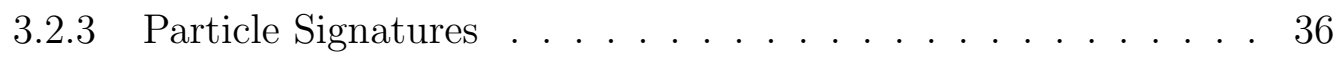

$3.2 .4 \quad$ Systematic Uncertainties . . . . . . . . . . . . . . . . . . . . 39

4 Event Simulation and Reconstruction 41

4.1 Monte Carlo Event Simulation . . . . . . . . . . . . . . . . . . . . . . 41

$4.1 .1 \quad$ NC $\Delta$ Radiative Modeling . . . . . . . . . . . . . . . . . 43

4.1 .2 Systematic Uncertainties . . . . . . . . . . . . . . . 47

4.2 Pandora Reconstruction . . . . . . . . . . . . . . . . . . . . . . . . . 48

4.3 Shower Kinematic Variables . . . . . . . . . . . . . . . . . . . . . . . 52

$4.3 .1 \quad$ Shower Energy $\ldots \ldots \ldots$. . . . . . . . . . . . . . . 52 
4.3 .2 Shower $\mathrm{dE} / \mathrm{dx}$. . . . . . . . . . . . . . . . . 53

4.4 Optical Flash Reconstruction . . . . . . . . . . . . . . . . . . . 54

5 Neutrino Induced NC $\Delta$ Radiative Event Selection 55

5.1 Vertex Reconstruction . . . . . . . . . . . . . . . . . . 55

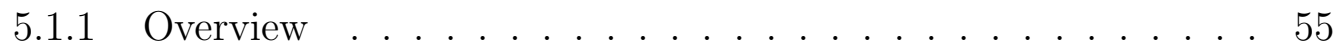

5.1 .2 Track Association . . . . . . . . . . . . . . . . . 5 56

5.1 .3 Shower Association . . . . . . . . . . . . . . . . . 58

5.1 .4 Reconstruction - Truth Matching . . . . . . . . . . . 66

$5.1 .5 \quad$ Optimization . . . . . . . . . . . . . . . . 67

5.2 Neutral Current $\Delta$ Radiative Topologies $\ldots \ldots$. . . . . . . . . . . . . 82

5.3 Monte Carlo and Data Samples . . . . . . . . . . . . . . . . . . . . . 82

5.4 Pre-selection . . . . . . . . . . . . . . . . . . . . . . . . . 85

5.5 Boosted Decision Tree Background Rejection . . . . . . . . . . . . . . 87

5.5.1 Training . . . . . . . . . . . . . . . . . . . . . . . . . 89

5.5.2 Cosmic Rejection Boosted Decision Tree . . . . . . . . . . . . 92

$5.5 .3 \quad$ BNB Rejection Boosted Decision Tree . . . . . . . . . . . . . 102

5.6 Training Variable Correlations . . . . . . . . . . . . . . . . . . . . 111

5.6 .1 Removal of Correlated Variables . . . . . . . . . . . . . . . . . 114

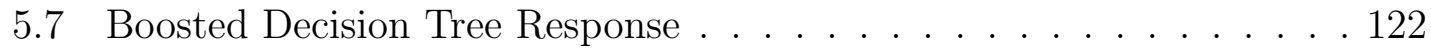

$\begin{array}{llr}6 & \text { Results } & 124\end{array}$

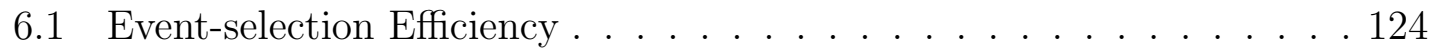

6.2 Background Composition . . . . . . . . . . . . . . . . . 128

6.3 Invariant $\Delta$ Mass $\ldots \ldots \ldots \ldots$. . . . . . . . . . . . . . 133

6.4 Data-Monte Carlo Comparison . . . . . . . . . . . . . . . . . 136

6.5 Systematic Uncertainties . . . . . . . . . . . . . . . . . . . . . . . . 148

6.6 Sensitivity to a NC $\Delta$ radiative Low Energy Excess $\ldots . . . . .149$

\begin{tabular}{lll}
\hline 7 & Conclusions & 152
\end{tabular} 
\begin{tabular}{ll}
\hline Appendices & 154
\end{tabular}

\begin{tabular}{|l|}
\hline A Cosmic-only Data-Monte Carlo Comparison \\
154
\end{tabular}

\begin{tabular}{lr}
\hline B MiniBooNE Flux Cross-checks & 161
\end{tabular}

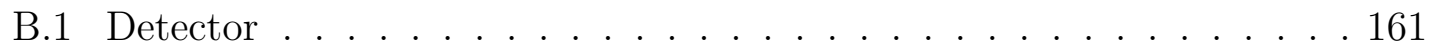

B.2 Neutrino Events . . . . . . . . . . . . . . . . . . . . . . . 162

B.3 Event Samples . . . . . . . . . . . . . . . . . . . . . . 163

B.4 $\nu_{\mu}$ CCQE Event Selection $\ldots \ldots \ldots$. . . . . . . . . . . . . . . . .

B.5 Data Cross-check . . . . . . . . . . . . . . . . . . . 166

B.6 Summary . . . . . . . . . . . . . . . . . . . . . . 170

\begin{tabular}{lr}
\hline References & 171
\end{tabular}

Word count: 33314 


\section{Abstract}

The MiniBooNE experiment is a Cherenkov based experiment located on the Booster Neutrino Beam. It observed an unexplained excess of $\nu_{e}$ charged current quasi-elastic (CCQE) event candidates in the reconstructed $E_{\nu}^{Q E} \approx 200-475 \mathrm{MeV}$ region known as the "Low Energy Excess" (LEE). These $\nu_{e}$ CCQE event candidates were in the form of lone showers, a signature left by both electrons and photons indistinguishably in the MiniBooNE detector. For this reason the identity of the MiniBooNE LEE as a photon or electron excess is currently unknown. Liquid argon time projection chambers (LArTPCs) are a relatively new type of detector able to distinguish between photons and electrons. The MicroBooNE detector is one such LArTPC and is situated on the same beamline and at a similar baseline as the MiniBooNE detector. This makes MicroBooNE an ideal experiment to test the photon and electron interpretations of the MiniBooNE LEE. This thesis presents the search for a photon LEE using an enhanced rate of neutrino-induced neutral current (NC) $\Delta$ production and subsequent radiative decay ( $\mathrm{NC} \Delta$ radiative) as the candidate source. A multi-stage NC $\Delta$ radiative event selection has been developed which takes full advantage of MicroBooNE's photon-electron discrimination capability. The ability of the search to select $\mathrm{NC} \Delta$ radiative events in MicroBooNE and validation of the search with $4.8 \times 10^{19}$ protons-on-target worth of data are presented. Also shown are preliminary studies of MicroBooNE's sensitivity to a $\mathrm{NC} \Delta$ radiative interpretation of the LEE. 


\section{Declaration}

No portion of the work referred to in this thesis has been submitted in support of an application for another degree or qualification of this or any other university or institute of learning.

This thesis contains the work of the author as part of the MicroBooNE collaboration as well as work from other members of the MicroBooNE collaboration and work from members of the MiniBooNE collaboration. Simulation and reconstruction in MicroBooNE were performed using LArSoft [1] to which many collaborators from multiple LArTPC experiments have contributed.

The development, testing and optimization of the vertex reconstruction algorithm described in Section 5.1 was performed solely by the author.

The analysis event selection strategy in Chapter 5 including pre-selection cuts and the boosted decision tree (BDT) selections were originally developed by the author. The boosted decision trees implemented in the selection are from the Toolkit for Multivariate Data Analysis [2]. Modifications and optimizations to the selection have since been made by both the author and postdoc Mark Ross-Lonergan (Columbia U.). These optimizations include the modification/addition/removal of various pre-selection cuts and BDT training variables. The reconstructed shower energy and $\mathrm{dE} / \mathrm{dx}$ calculation methods used in the analysis were originally developed by graduate student Roberto Stefano Soleti (Havard U.). Figures 6.20 and 6.21 were produced by Mark Ross-Lonergan.

Hand scanning to determine the relative contributions to $\pi^{0}$ background listed 
in Table 6.5 was performed by NSF REU student Aileen Zhai (Columbia U.) and undergraduate student Keng Lin (Columbia U.) [3].

The MiniBooNE cross-checks described in Chapter B were performed by the author using the $\nu_{\mu}$ CCQE analysis selection developed by (previously) graduate student Joe Grange (Argonne National Laboratory) [4]. The MiniBooNE data files used for this cross-check were processed by both Joe Grange and Zarko Pavlovic (Fermilab, Neutrino division). 


\section{Copyright Statement}

The author of this thesis (including any appendices and/or schedules to this thesis) owns certain copyright or related rights in it (the "Copyright") and s/he has given The University of Manchester certain rights to use such Copyright, including for administrative purposes.

Copies of this thesis, either in full or in extracts and whether in hard or electronic copy, may be made only in accordance with the Copyright, Designs and Patents Act 1988 (as amended) and regulations issued under it or, where appropriate, in accordance with licensing agreements which the University has from time to time. This page must form part of any such copies made.

The ownership of certain Copyright, patents, designs, trademarks and other intellectual property (the "Intellectual Property") and any reproductions of copyright works in the thesis, for example graphs and tables ("Reproductions"), which may be described in this thesis, may not be owned by the author and may be owned by third parties. Such Intellectual Property and Reproductions cannot and must not be made available for use without the prior written permission of the owner(s) of the relevant Intellectual Property and/or Reproductions.

Further information on the conditions under which disclosure, publication and commercialisation of this thesis, the Copyright and any Intellectual Property and/or Reproductions described in it may take place is available in the University IP Policy (see http://documents.manchester .ac.uk/DocuInfo. aspx?DocID=24420), in any relevant Thesis restriction declarations deposited in the University Library, The University Library's regulations (see http://www.library.manchester.ac. 
uk/about/regulations/) and in The University's policy on Presentation of Theses. 


\section{Acknowledgments}

I would like to thank my Ph.D. advisor, Dr. Georgia Karagiorgi, for her support and patience in guiding me through my research. Thank you for working so hard to ensure I had everything I needed to succeed and grow. I couldn't have done it without you.

I would also like to thank my advisor Prof. Stefan Söldner-Rembold for his advice and direction during my time at the University of Manchester.

My thanks to all the people I met at both the University of Manchester and Fermilab. The conversations we've had made my time as a Ph.D. student instructive as well entertaining. In particular I would like to thank the people willing to take time out of their day to ensure I understood a physics concept or a tricky piece of code. Your help was much appreciated.

Finally, I would like to thank my parents who have offered me endless support, kindness and love. I'm very lucky to have you. 


\section{Introduction}

The Mini Booster Neutrino Experiment (MiniBooNE) is an accelerator based neutrino experiment located on the Booster Neutrino Beam (BNB) at the Fermi National Accelerator Laboratory (Fermilab) and uses a Cherenkov detector to study BNB $\stackrel{(-)}{\nu}$ and $\stackrel{(-)}{\nu_{e}}$ interactions. The primary physics goal of MiniBooNE was to investigate an anomaly observed by the Liquid Scintillator Neutrino Detector (LSND) experiment: an excess of $\bar{\nu}_{e}$ event candidates in a $\bar{\nu}_{\mu} \rightarrow \bar{\nu}_{e}$ search. MiniBooNE observed a similar anomalous excess of $\nu_{e}$ CCQE event candidates in the reconstructed $E_{\nu}^{Q E} \approx 200-475 \mathrm{MeV}$ region known as the MiniBooNE "Low Energy Excess" (LEE). These event candidates were identified by searching for the electron produced in the $\nu_{e}$ CCQE interaction.

Electrons and photons both produce electromagnetic showers that are indistinguishable in MiniBooNE but are not ultimately identical. An electron shower will begin with the electron itself, whereas a photon shower will begin by pair-producing an electron-positron pair. MiniBooNE detects particles primarily via Cherenkov photons, whose emission is azimuthally symmetric about the particle's direction. This produces a ring-like signature in the detector that can be used to determine the particle's identity, energy, direction and other qualities. As MiniBooNE detects showers (and other particles) via Cherenkov rings, the initial difference between photon and electron showers cannot be observed. This gives photon and electron showers identical signatures in MiniBooNE. The identity of the LEE as a true $\nu_{e}$ CCQE event excess or an anomalous photon process is therefore unknown.

There are many potential interpretations of the MiniBooNE LEE as either photon or electron. The most popular interpretation is an electron LEE observed due to the existence of non-weakly interacting (sterile) neutrinos that alter standard threeneutrino oscillations, enhancing the probability of $\nu_{\mu} \rightarrow \nu_{e}$ transition and thus the rate of observed $\nu_{e} \mathrm{CCQE}$ interactions in MiniBooNE [5-8]. An equally valid theory is a photon LEE observed due to the mis-estimation of single-photon backgrounds in MiniBooNE [9] or an entirely new neutrino-induced single-photon process [10]. MiniBooNE's inability to distinguish between photon and electron showers means it has been unable to investigate the identity of the LEE.

The development of neutrino detectors using liquid argon time projection cham- 
bers (LArTPCs) presents a new opportunity to study the MiniBooNE LEE. A LArTPC can reconstruct neutrino interactions via the ionisation of target material induced by charged particles produced by the neutrino interaction. These ionisation electrons are drifted by a uniform electric field to an anode where they are collected. Collection of ionisation electrons allows for the reconstruction of the path charged particles have traversed through the detector as well as the energy deposited along that path. This includes the initial particles of photon and electron showers. As the start of a photon shower is comprised of two particles (an electron-positron pair), the rate of energy deposition at the start of the shower will be roughly double that of an electron shower, which has only one particle at the start. Reconstruction of the rate of energy deposition across the start of the shower will enable a LArTPC to distinguish between photon and electron showers and therefore makes it a detector well suited to determining the identity of the LEE.

The Micro Booster Neutrino Experiment (MicroBooNE) is a LArTPC experiment situated on the same beamline and at a similar baseline to the MiniBooNE experiment. MicroBooNE's primary physics goals are to determine the identity of the LEE as well as to perform measurements of low energy neutrino cross-sections on argon. This thesis describes the development of a search for a photon LEE in MicroBooNE under the hypothesis that a mis-estimation of the LEE photon background was the cause of the LEE observation. This search considers an enhanced rate of neutrino-induced $\mathrm{NC}$ resonant $\Delta$ production and subsequent radiative decay (henceforth $\mathrm{NC} \Delta$ radiative decay), one of the most significant photon backgrounds to the LEE, as the candidate LEE source.

Chapter 2 gives a basic overview of standard three-neutrino oscillation theory and current best fit parameters, a description of the MiniBooNE LEE, its predicted photon backgrounds, constraints and the main LEE interpretations. A description of the neutrino-induced NC $\Delta$ radiative process is also included. Chapter 3 describes the MicroBooNE experiment. This includes the Booster Neutrino Beam (BNB) which is the neutrino beam both MiniBooNE and MicroBooNE are located on [11], the operating principles of LArTPCs, the MicroBooNE detector and the particle signatures expected in the detector. Chapter 4 describes the simulation of neutrino interactions in the MicroBooNE detector, the modeling of neutrino-induced NC $\Delta$ radiative decay, the automated reconstruction of MicroBooNE data and methods used to determine some reconstructed kinematic shower variables. Chapter 5 is a description of the multi-stage neutrino-induced NC $\Delta$ radiative decay selection process developed for MicroBooNE. Chapter 6 contains the performance of the event selection on simulated neutrino events, a data-Monte Carlo (MC) comparison 
between selected simulated events and the $4.8 \times 10^{19}$ protons on target (POT) of unblinded data currently available from MicroBooNE and the expected stats-only sensitivity of MicroBooNE to a NC $\Delta$ radiative decay produced LEE.

From 2003-2008 MiniBooNE took the data that would be used to observe the LEE. In November 2015 the MiniBooNE experiment started taking new data. In order to ensure consistency between on the old LEE data and the new data being taken, various cross-checks were performed between these old and new datasets. Consistency in the BNB is extremely important for the MicroBooNE experiment as any changes since the MiniBooNE LEE was observed could affect the measurement of a LEE in MicroBooNE. Appendix B contains work performed by the author to this end. 


\section{Physics Motivation}

\section{$2.1 \quad$ Overview}

\subsubsection{Discovery of Neutrino Flavour Transitions}

Excluding certain anomalies, the model that best describes current neutrino data is the standard three-neutrino model. According to this model there are three neutrino flavours: $\nu_{e}, \nu_{\mu}$ and $\nu_{\tau}$ and these flavours mix with each other, allowing a neutrino of one flavour to transition into another. Neutrino flavour transitions were first encountered in the form of the "solar neutrino problem" [12], where experiments sensitive to the solar $\nu_{e}$ flux detected roughly a third of the $\nu_{e}$ s predicted by the solar models at that time. The Homestake experiment, which used a chlorine based detector to detect the solar $\nu_{e}$ flux through the charged current (CC) $\mathrm{Cl}+\nu_{e} \rightarrow \mathrm{Ar}+e^{-}$interaction, was the first to observe this $\nu_{e}$ deficit 13. The flux predictions of the solar models at that time were in fact correct, however due to neutrino flavour transitions induced by matter effects in the sun, two thirds of the solar $\nu_{e} \mathrm{~s}$ arrived as $\nu_{\mu} \mathrm{s}$ or $\nu_{\tau} \mathrm{s}$ in the Homestake detector. Solar $\nu_{e} \mathrm{~s}$ are typically produced with energies of $\mathcal{O}(1-10) \mathrm{MeV}$, giving them more energy than the electron mass but less than the muon or tau masses. The detector's reliance on CC interactions to observe neutrinos meant that, due to the low energy of the solar neutrinos, only electrons could be produced by CC solar neutrino interactions. No visible leptons could be produced by $\mathrm{CC} \nu_{\mu}$ or $\nu_{\tau}$ interactions and thus only $\nu_{e} \mathrm{~s}$ were observed. The Sudbury Neutrino Observatory (SNO) 14 later observed the total solar neutrino flux by using heavy water as its detector medium, allowing neutrinos to interact via $\mathrm{NC}$ interactions. In $\mathrm{NC}$ neutrino interactions no charged lepton is produced and so $\mathrm{NC} \nu_{\mu}$ and $\nu_{\tau}$ interactions are just as viable as $\mathrm{NC} \nu_{e}$ interactions. This allowed all neutrino types to interact in the SNO detector and consequently allowed for the observation of the total solar neutrino flux. SNO's observation of the total solar neutrino flux was the first clear evidence of neutrino flavour transitions.

Additional experiments that produced results consistent with the standard threeneutrino model include the Large Electron-Positron Collider, which measured the number of weakly-active light neutrinos, $N_{\nu}$, through measurement of the $Z^{0}$ decay width, finding $N_{\nu}=2.9840 \pm 0.0082$ [15]. Evidence for oscillation of atmospheric neutrinos was observed by Super Kamiokande [16] which found a deficit in upward 
moving atmospheric $\nu_{\mu} \mathrm{s}$ compared to the downward moving $\nu_{\mu} \mathrm{s}$. The upwards moving $\nu_{\mu} s$ were produced in the atmosphere on the opposite side of the Earth and travelled through the Earth to reach the detector, while the downward facing $\nu_{\mu} s$ were produced in the atmosphere above the detector. As will be described in the following section, the distance travelled by the upward facing $\nu_{\mu} \mathrm{s}$ was large enough for a portion of them to transition into $\nu_{\tau} \mathrm{s}$ though the phenomenon of neutrino oscillation, producing the observed deficit.

\subsubsection{Global Three Neutrino Oscillation Picture}

According to the standard three-neutrino model each neutrino flavour does not have a single mass eigenstate associated with it but instead exists as a superposition of three mass eigenstates $\nu_{1}, \nu_{2}$ and $\nu_{3}$ with masses $m_{1}, m_{2}$ and $m_{3}$ respectively. The relation between neutrino flavour states and mass states can be described using

$$
\left|\nu_{\alpha}\right\rangle=\sum_{j} U_{\alpha j}^{*}\left|\nu_{j}\right\rangle
$$

where $\left|\nu_{\alpha}\right\rangle$ is the time-independent flavour state $(\alpha=e, \mu, \tau),\left|\nu_{j}\right\rangle$ is the timeindependent mass state $(\mathrm{j}=1,2,3)$ and $U_{\alpha j}$ is the Pontecorvo-Maki-NakagawaSakata (PMNS) matrix [17]. The PMNS matrix is a $3 \times 3$ unitary matrix that describes the relation of neutrino flavour states and mass eigenstates. It is parameterized by the three mixing angles $\theta_{12}, \theta_{13}$ and $\theta_{23}$ as well as a charge-parity violating phase factor $\delta$. Each mixing angle is a measure of how strongly two mass eigenstates mix with each other. The PMNS matrix is conventionally written as

$$
\begin{aligned}
U & =\left(\begin{array}{ccc}
1 & 0 & 0 \\
0 & c_{23} & s_{23} \\
0 & -s_{23} & c_{23}
\end{array}\right)\left(\begin{array}{ccc}
c_{13} & 0 & s_{13} e^{-i \delta} \\
0 & 1 & 0 \\
-s_{13} e^{i \delta} & 0 & c_{13}
\end{array}\right)\left(\begin{array}{ccc}
c_{12} & s_{12} & 0 \\
-s_{12} & c_{12} & 0 \\
0 & 0 & 1
\end{array}\right) \\
& =\left(\begin{array}{ccc}
c_{12} c_{13} & s_{12} c_{13} & s_{13} e^{-i \delta} \\
-s_{12} c_{23}-c_{12} s_{23} s_{13} e^{i \delta} & c_{12} c_{23}-s_{12} s_{23} s_{13} e^{i \delta} & s_{23} c_{13} \\
s_{12} s_{23}-c_{12} c_{23} s_{13} e^{i \delta} & -c_{12} s_{23}-s_{12} c_{23} s_{13} e^{i \delta} & c_{23} s_{13}
\end{array}\right)
\end{aligned}
$$

where $c_{j k}=\cos \theta_{j k}$ and $s_{j k}=\sin \theta_{j k} . \theta_{j k}$ is the mixing angle between the mass eigenstates $j$ and $k$. When a neutrino of a specific flavour is produced and travels, its mass eigenstates will progress at different rates due to their differing masses. Due to this change in mass eigenstate superposition, the flavour of the neutrino observed some distance away from its source will not necessarily be the same as the flavour at production. The probability that such a neutrino flavour transition occurs is not constant and will oscillate based on a variety of factors. 
The oscillation of neutrino transition probability for the standard three-neutrino model can in many cases be reduced to a two-neutrino oscillation model. In this model there are only two neutrino flavour states, $\nu_{\alpha}$ and $\nu_{\beta}$, and two corresponding mass eigenstates $\nu_{1}$ and $\nu_{2}$. The probability a neutrino produced in the flavour state $\nu_{\alpha}$ being observed at some later point as a different flavour state $\nu_{\beta}$ is given by

$$
\begin{aligned}
P\left(\nu_{\alpha} \rightarrow \nu_{\beta \neq \alpha}\right) & =\left|\left\langle\nu_{\beta}(x) \mid \nu_{\alpha}\right\rangle\right|^{2} \\
& =\left|c_{12} s_{12}\left(e^{i p_{2} \cdot x}-e^{i p_{1} \cdot x}\right)\right|^{2} \\
& =\sin ^{2} 2 \theta_{12} \sin ^{2}\left(\frac{\left(p_{2}-p_{1}\right) \cdot x}{2}\right),
\end{aligned}
$$

where $x$ is the position of the neutrino in space-time and $p_{j=1,2}$ is the four-momentum of a given mass eigenstate. Assuming a relativistic neutrino whose mass eigenstates' energies $E_{1}$ and $E_{2}$ are approximately equal gives

$$
P\left(\nu_{\alpha} \rightarrow \nu_{\beta \neq \alpha}\right)=\sin ^{2} 2 \theta_{12} \sin ^{2}\left(\frac{\Delta m_{21}^{2} L}{4 E}\right)
$$

where $\Delta m_{21}^{2}=m_{2}^{2}-m_{1}^{2}$ is defined as the mass splitting between the two neutrino mass eigenstates, $L$ is the distance travelled by the neutrino and $E_{1} \approx E_{2} \approx E$. This equation shows that the probability for a neutrino to undergo flavour transition oscillates as the neutrino travels. The amplitude of this probability oscillation is dictated by the mixing angle and the frequency of oscillation is dependent on the mass splitting, neutrino energy and distance travelled by the neutrino. The oscillatory nature of neutrino flavour transition probability is very well illustrated by the results of the KamLAND reactor neutrino experiment as can be seen in Figure 2.1. The majority of neutrino (and anti-neutrino) oscillation data is consistent with the three neutrino oscillation picture with three independent mixing angles and two independent mass splittings. A summary of the combined best-fit three neutrino mixing angles and mass splittings using data from multiple neutrino experiments is listed in table 2.1.

\begin{tabular}{|l|c|}
\hline Parameter & Value \\
\hline $\sin ^{2} \theta_{12}$ & $0.307_{-0.012}^{+0.013}$ \\
\hline $\sin ^{2} \theta_{23}$ (quadrant I) & $0.417_{-0.028}^{+0.025}$ \\
\hline $\sin ^{2} \theta_{23}$ (quadrant II) & $0.597_{-0.030}^{+0.024}$ \\
\hline $\sin ^{2} \theta_{13}$ & $(2.12 \pm 0.08) \times 10^{-2}$ \\
\hline$\Delta m_{21}^{2}$ & $(7.53 \pm 0.18) \times 10^{-5} \mathrm{eV}^{2}$ \\
\hline$\left|\Delta m_{23}^{2}\right|$ & $(2.51 \pm 0.05) \times 10^{-3} \mathrm{eV}^{2}$ \\
\hline
\end{tabular}

Table 2.1: Measured three neutrino oscillation best-fit three neutrino mixing angles and mass splittings from the 2018 Particle Data Group review [19]. 


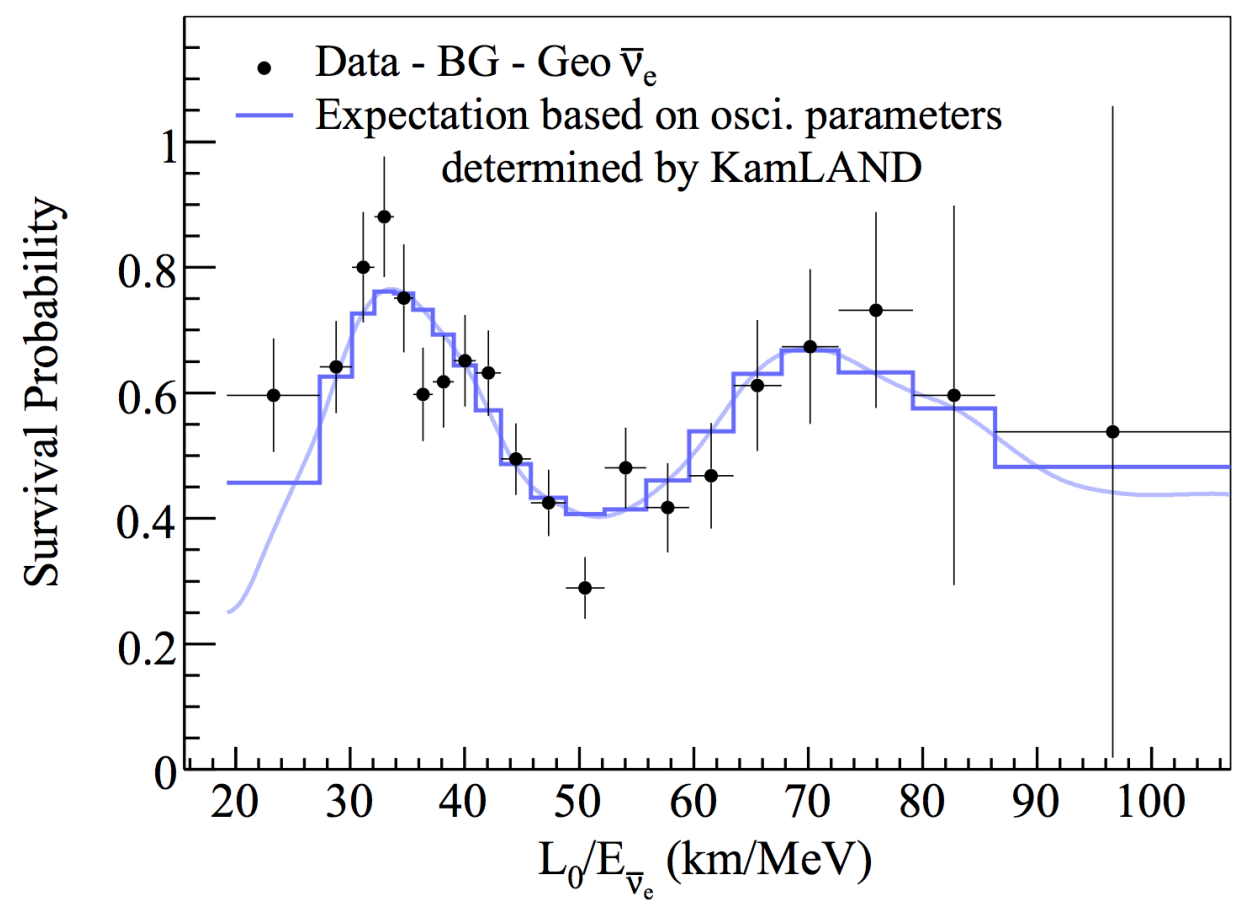

Figure 2.1: Results from the KamLAND reactor neutrino experiment showing the oscillatory changes in survival probability of $\bar{\nu}_{e}$ from multiple reactors in terms of average reactor-to-detector distance divided by anti-neutrino energy, $L_{0} / E_{\bar{\nu}}$ [18]. The continuous curve shows the un-binned survival probability the expected survival probability histogram is derived from.

\subsection{The MiniBooNE Low Energy Excess}

MiniBooNE [20] is an accelerator-based neutrino experiment located on the BNB at Fermilab and is a direct precursor to MicroBooNE. MiniBooNE uses a Cherenkov based detector with a mineral oil neutrino target and was motivated by the Liquid Scintillator Neutrino Detector (LSND) experiment [21]. LSND was an accelerator based neutrino experiment in the $\Delta m^{2} \approx 1 \mathrm{eV}^{2}$ mass splitting region that observed an excess of $\bar{\nu}_{e}$ event candidates in a $\bar{\nu}_{\mu} \rightarrow \bar{\nu}_{e}$ search. In order to probe the same mass splitting region as LSND, the MiniBooNE detector was placed $541 \mathrm{~m}$ from the BNB source. For accelerator experiments studying standard three-neutrino flavour transitions, the low mass splitting values (see Table 2.1) dictate the detector be a distance of $\mathcal{O}(100-1000) \mathrm{km}$ from the beam source to maximise the neutrino transition probability for the flavour transition of interest. These kinds of experiments are known as long baseline experiments. Unlike long-baseline experiments, the mass splitting region of interest for LSND and MiniBooNE is comparatively much larger at $\Delta m^{2} \approx 1 \mathrm{eV}^{2}$. For this reason the LSND and MiniBooNE detectors were placed much closer to their respective beam sources than the typical accelerator based neu- 
trino experiments. Experiments such as LSND and MiniBooNE are known as short baseline (SBL) experiments.

At this baseline MiniBooNE was designed to search for $\nu_{\mu} \rightarrow \nu_{e}$ and $\bar{\nu}_{\mu} \rightarrow \bar{\nu}_{e}$ transitions and detected an excess of $\nu_{e}$ CC quasi-elastic (CCQE) event candidates. This excess consisted of $78.4 \pm 28.5 \nu_{e}$ CCQE event candidates in the $200<E_{\nu}^{Q E}<$ $1250 \mathrm{MeV}$ energy range with a significance of $2.8 \sigma[22]$. MiniBooNE has taken further data since its first LEE observation and has recently released new results: an excess of $381.2 \pm 85.2 \nu_{e} \mathrm{CCQE}$ events in the $200<E_{\nu}^{Q E}<1250 \mathrm{MeV}$ energy range with a significance of $4.5 \sigma[23]$. For the purposes of this thesis only neutrino mode data from the original LEE observation are considered. The events that contribute to the LEE consist of a single shower and no other visible final states in the detector volume. MiniBooNE is unable to distinguish between showers produced by electrons and showers produced by photons, so the identity of the LEE as photon or electron (or some combination) is unknown.

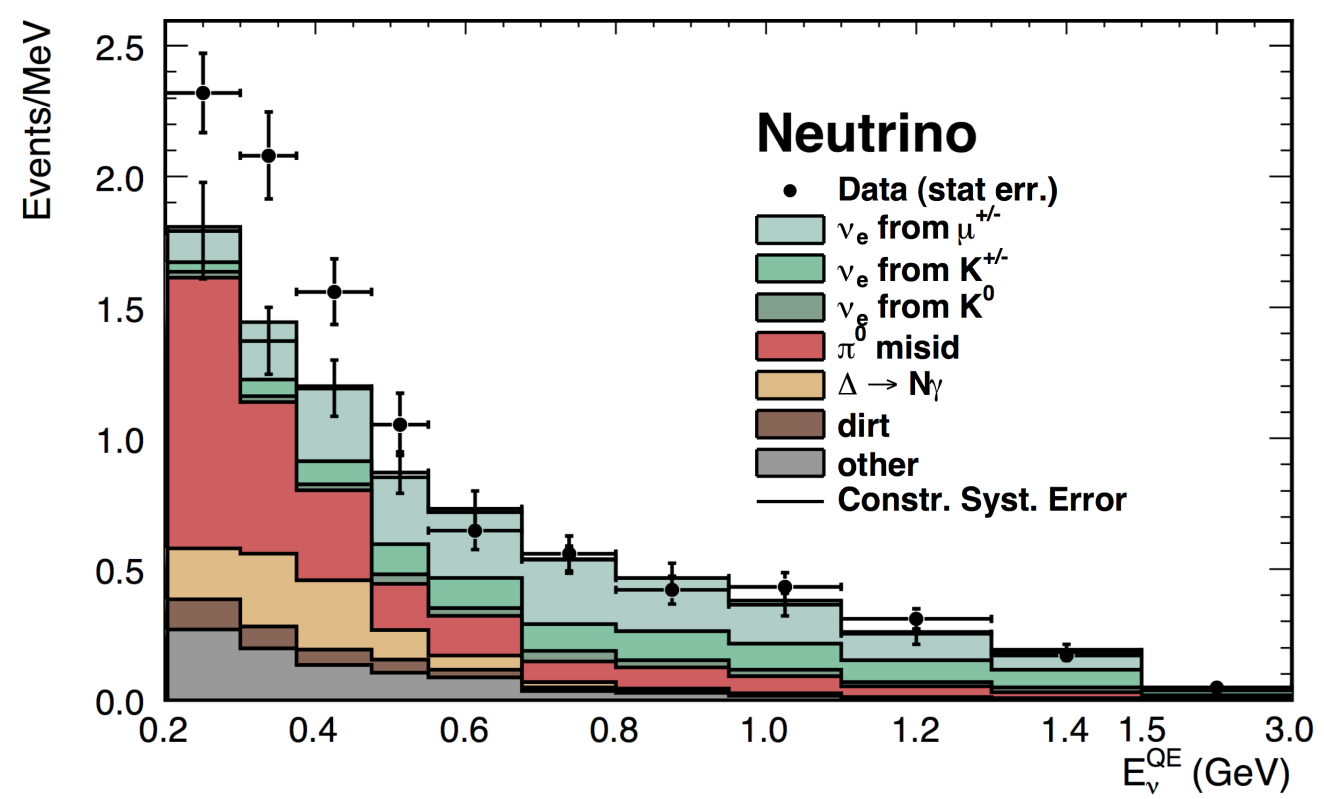

Figure 2.2: The MiniBooNE low energy excess of $\nu_{e}$ CCQE event candidates and predicted backgrounds from neutrino running mode in terms of reconstructed neutrino energy assuming a $\nu_{e}$ CCQE interaction [22].

Figure 2.2 shows the MiniBooNE LEE observed data and predicted background in terms of the reconstructed neutrino energy assuming a $\nu_{e}$ CCQE interaction, $E_{\nu}^{Q E}$, given by

$$
E_{\nu}^{Q E}=\frac{m_{p}^{2}-m_{n}^{2}-m_{e}^{2}+2 m_{n} E_{v i s}}{2\left(m_{n}-E_{v i s}+\cos \theta \sqrt{E_{v i s}^{2}-m_{e}^{2}}\right)},
$$


where $m_{p}$ is the mass of a proton, $m_{n}$ is the mass of a neutron, $m_{e}$ is the mass of an electron, $E_{v i s}$ is the visible energy of the interaction (i.e. the energy the lone shower deposited in the detector) and $\theta$ is the angle between the direction of the shower and the BNB axis. A significant portion of the background to the MiniBooNE LEE is from NC photon related processes. The NC nature of these processes means that they also inhabit the same low energy region as the LEE.

The largest photon background contribution for the LEE search is from NC $\pi^{0} \rightarrow 2 \gamma$ decay (the red contribution in Figure 2.2). Should only one of the decay photons be observed (the other photon escapes the detector volume prior to converting into a shower, is not properly reconstructed, etc) and no other visible final states are present in the detector, the signature will be a single lone shower mimicking the signature of the LEE. The predicted NC $\pi^{0}$ background is constrained in MiniBooNE by a direct measurement of the $\mathrm{NC} \pi^{0}$ rate [24]. For this measurement $\pi^{0} \mathrm{~s}$ were selected by searching for two shower events with an invariant mass compatible with the $\pi^{0}$ mass $\left(135 \mathrm{MeV} / \mathrm{c}^{2}\right)$ and purity was ensured by discarding events with a reconstructed $\pi^{0}$ mass outside a $80-200 \mathrm{MeV} / \mathrm{c}^{2}$ range. A comparison between MiniBooNE NC $\pi^{0} \mathrm{MC}$ and this data measurement was used to create a $\mathrm{NC} \pi^{0}$ correction function used to bring the MC NC $\pi^{0}$ background in the LEE search into agreement with the rate predicted by the data measurement. Applying the correction function on the LEE NC $\pi^{0}$ background results in a $13 \%$ increase in the $E^{Q E}<400 \mathrm{MeV}$ (signal) energy region and a $20 \%$ decrease above this energy. The uncertainty on the NC $\pi^{0}$ background after correction is $7 \%$. A mis-estimated $\mathrm{NC} \pi^{0}$ background has been previously considered as a potential source of the LEE, however studies found an enhancement factor of 2.0 would be required for the background to match the magnitude of the LEE. This would be a $5 \sigma$ deviation from the data-constrained expected NC $\pi^{0}$ background [25].

"Dirt" events are neutrino interactions outside the detector that produce activity inside the detector. A photon produced from such an event that converts inside the detector (e.g. a photon from $\pi^{0} \rightarrow 2 \gamma$ decay) will appear as an LEE signature shower and thus also contributes to the background (the brown contribution in Figure 2.2. The LEE dirt background is constrained by a direct measurement of the dirt rate in MiniBooNE [26]. Events with activity close to the detector boundaries and inward-facing tracks (i.e. events that appeared to have activity from outside the detector) were selected to make a measurement on the rate. A comparison between the MiniBooNE dirt MC and the dirt measurement was used to create a flat normalization factor with which the MC LEE dirt background is constrained. For neutrino mode this normalization factor was calculated to be $0.7 \pm 0.1$ with a 
$0.15 \times E_{\nu}^{Q E}$ uncertainty 25$]$.

The $\Delta \rightarrow N+\gamma$ background (NC $\Delta$ radiative decay) is discussed in detail in the following section.

\subsection{Interpretations of the Low Energy Excess}

\subsubsection{Neutral Current $\Delta$ Radiative Decay}

MiniBooNE's inability to distinguish between electrons and photons means any neutrino interaction that produces a single photon and no other visible final states is a candidate source for the LEE. The analysis presented in this thesis considers the photon interpretation of the MiniBoone LEE using an enhanced rate of neutrinoinduced NC resonant $\Delta$ production and subsequent radiative decay as the candidate source. This process was chosen as it produces the same signature as the LEE, is a single photon process that resides in the same energy region as the LEE, was an irreducible background for the LEE (the tan contribution in Figure 2.2) and no constraints currently exist on its cross-section that would prevent it from being the sole contributor to the LEE. The full interaction occurs via the following successive processes:

$$
\begin{aligned}
\nu+N & \rightarrow \nu+\Delta(\text { resonant } \Delta \text { production }) \\
\Delta & \rightarrow N+\gamma(\Delta \text { radiative decay }),
\end{aligned}
$$

where $\nu$ is a neutrino of any type, $N$ is a nucleon, $\Delta$ is a Delta resonance and $\gamma$ is a photon. Figure 2.3 illustrates this process. An incoming neutrino undergoes a $\mathrm{NC}$ interaction with a nucleon in the detector target material, producing a either a $\Delta^{+}$(uud) or $\Delta^{0}$ (udd) baryon (or corresponding anti-particles). $\Delta$ baryons have a lifetime of $\mathcal{O}\left(10^{-24}\right)$ seconds and will decay rapidly after production. The largest branching ratio of $\Delta$ decay, at $99.4 \%$, corresponds to $\Delta \rightarrow N+\pi$. The remaining $0.6 \pm 0.05 \%$ corresponds to radiative decay, where the $\Delta$ resonance will decay into a photon and corresponding nucleon $\left(\Delta^{+} \rightarrow\right.$ proton, $\Delta^{0} \rightarrow$ neutron). A NC neutrino interaction may also produce a $\Delta^{++}$(uuu) or $\Delta^{-}$(ddd), however these baryon states cannot radiatively decay as they have no corresponding stable nucleon to decay to. To date there has been no observation of NC neutrino induced $\Delta \rightarrow N+\gamma$ decay and the branching ratio listed above are estimates from the Particle Data Group (PDG) 19].

While there has not yet been an observation of neutrino induced NC $\Delta$ radia- 


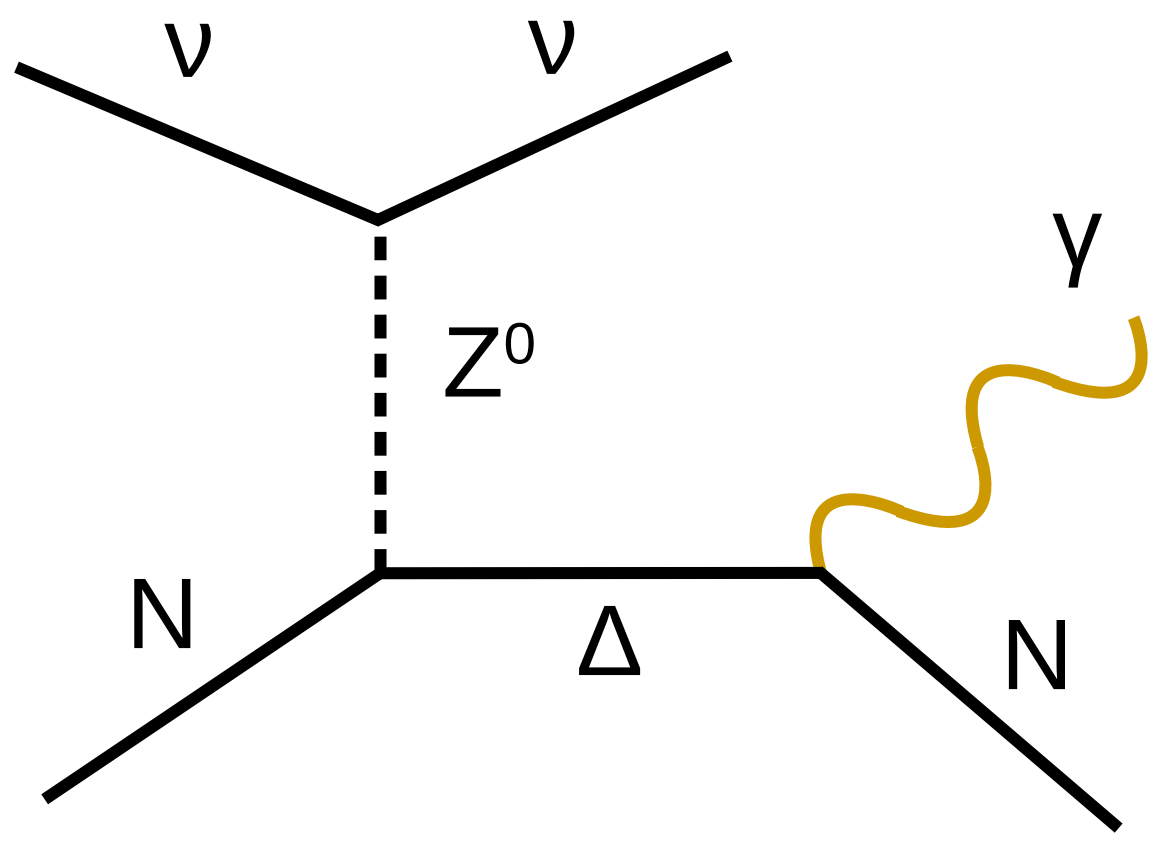

Figure 2.3: A NC resonant interaction between a neutrino, $\nu$ and a nucleon, $N$, produces a $\Delta$ baryon. The $\Delta$ baryon radiatively decays into a photon, $\gamma$, and a nucleon of the same type that was originally excited by the neutrino.

tive decay, two searches for neutrino-induced single photon production have been performed. The first was an inclusive single photon search performed in NOMAD (neutrino oscillation magnetic detector) at neutrino energies $E_{\nu} \approx 25 \mathrm{GeV}$ [27] (far above the energies of MiniBooNE and the LEE). The second was a NC single photon search performed using the T2K (Tokai to Kamioka) near detector (ND280) at neutrino energies $E_{\nu} \approx 0.6 \mathrm{GeV}$. This search placed a neutrino-induced single photon cross-section limit of $0.0903 \times 10^{-38} \mathrm{~cm}^{2} /$ nucleon at $90 \% \mathrm{CL}$ [28. This limit is well above the cross-section required for a $\mathrm{NC} \Delta$ radiative LEE as will be discussed in Section 4.1.1.

In MiniBooNE a NC $\Delta^{0} \rightarrow n+\gamma$ radiative decay produces a single visible photon shower with no other activity. A NC $\Delta^{+} \rightarrow p+\gamma$ radiative decay will also produce a single visible shower and no other activity as most protons produced by this decay will be below MiniBooNE's $350 \mathrm{MeV}$ Cherenkov threshold [29]. Given that the majority of resonant $\pi^{0}$ production in MiniBooNE is from $\Delta \rightarrow N+\pi$ and that this decay mode and $\Delta \rightarrow N+\gamma$ are both decays of the $\Delta$, the $\Delta \rightarrow N+\gamma$ background can be (and is) constrained by the same correction function used to constrain the NC $\pi^{0}$ background. The uncertainty on the LEE $\Delta \rightarrow N+\gamma$ background after the 
correction function has been applied is $12 \%$. This uncertainty arises from the $7 \%$ uncertainty on the $\Delta \rightarrow N+\gamma$ branching fraction and a $10 \%$ uncertainty of the $\pi$ escape probability on ${ }^{12} \mathrm{C}$. A mis-estimated $\mathrm{NC} \Delta \rightarrow N+\gamma$ background itself has been previously considered as a potential source of the LEE, however studies found an enhancement factor of 2.7 would be required for the background to match the magnitude of the LEE. This would be a $5 \sigma$ deviation from expected background [25].

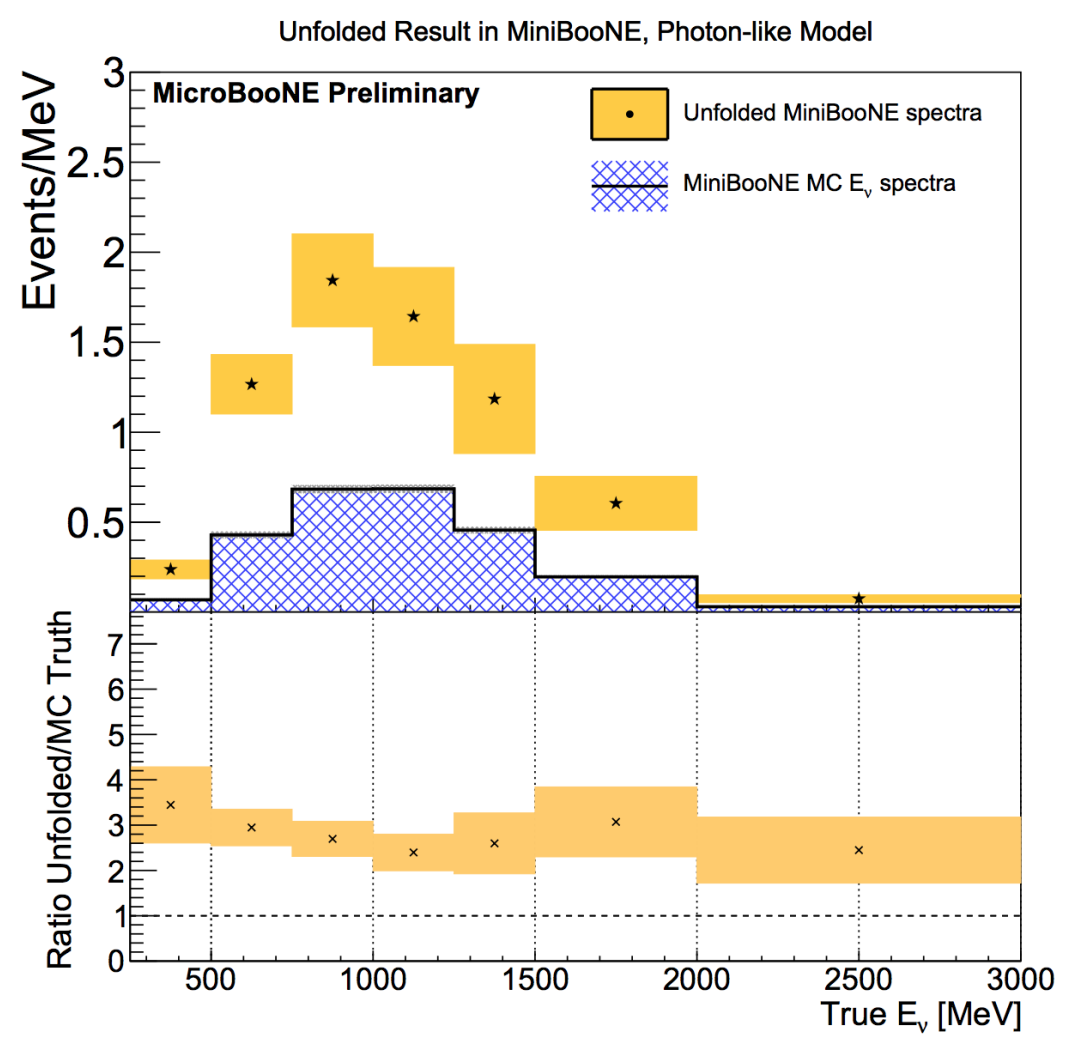

Figure 2.4: An unfolding of the MiniBooNE LEE under the photon-like NC resonant $\Delta$ production, with subsequent radiative decay hypothesis [30]. The top plot shows the unfolded photon-like LEE "true" neutrino energy superimposed with the MC NC $\Delta$ radiative true neutrino energy. The bottom plot shows the ratio of unfolded LEE to MC.

In order to test a LEE NC $\Delta$ radiative hypothesis, the effects of the MiniBooNE detector such as reconstruction and event selection must be "unfolded" in order to estimate the "true" MiniBooNE LEE NC $\Delta$ radiative signal. This is necessary to produce a corresponding MC signal estimation in the MicroBooNE detector. Such an unfolding was performed under a LEE NC $\Delta$ radiative hypothesis using MiniBooNE neutrino mode $\mathrm{MC}$ and $6.46 \times 10^{20}$ POT of data used in the LEE neutrino mode analysis [22]. Figure 2.4 shows the unfolded LEE data and MiniBooNE MC $\mathrm{NC} \Delta$ radiative decay both in terms of true neutrino energy. Also included is the 
ratio between these two distributions which is relatively uniform in terms of true neutrino energy. This ratio shows a roughly uniform three-fold increase in the normalization of the standard-model-predicted NC $\Delta$ radiative decay cross section is required to match the size of the LEE in MiniBooNE [30]. This roughly matches the 2.7 enhancement factor found in the study mentioned in Section 2.2. For the analysis in this thesis the LEE enhancement is applied as a flat $\times 3$ normalization factor. Only statistical uncertainties were considered in the unfolding.

\subsubsection{Sterile Neutrinos}

One potential source of the LEE is the source MiniBooNE was designed to search for: an enhanced rate of $\nu_{e}$ CCQE interactions due to the existence of one or more additional neutrino mass eigenstates corresponding to one or more additional non-weakly interacting (sterile) neutrino flavour states [5] 8 . $\nu_{e}$ CCQE interactions produce an electron and proton in their final state and while an electron is likely to convert into a visible shower in the MiniBooNE detector, a proton will typically be under Cherenkov threshold and therefore go undetected. In MiniBooNE such an interaction produces a detector lone shower signature identical to that of the observed LEE. This is the signature MiniBooNE was specifically designed to observe in its search for anomalies in the same $\Delta m^{2} \approx 1 \mathrm{eV}^{2}$ region as LSND. The existence of new, heavier, mass eigenstates $\left(m_{4}, m_{5}, \ldots\right)$ corresponding to sterile flavour states (henceforth sterile mass eigenstates) would alter the standard three-neutrino oscillation model assuming they were part of the $\nu_{e}, \nu_{\mu}$ or $\nu_{\tau}$ flavour state composition, enhancing the probability of $\nu_{\mu} \rightarrow \nu_{e}$ and $\bar{\nu}_{\mu} \rightarrow \bar{\nu}_{e}$ flavour transitions in the higherthan-standard mass-splitting regions. The latest combined neutrino-anti-neutrino MiniBooNE data places the best fit point for the mass splitting between the standard neutrino mass eigenstates and a sterile mass eigenstate in the $3+1$ ( 3 standard and 1 sterile) neutrino model at $0.041 \mathrm{eV}^{2}$ [23], a mass splitting considerably larger than the standard three-neutrino mass splitting values (see Table 2.1).

The sterile neutrino interpretation of the LEE has been studied in the past by considering the results of various SBL experiments but tension between these results under this interpretation is high 3134 . Such studies have considered the MiniBooNE LEE with results from other neutrino experiments sensitive to high $\Delta m^{2}$ oscillations. These include $\nu_{\mu}$ disappearance results from experiments such as CDHS 35] and MINOS [36, 37], which have seen no evidence for such oscillations, $\bar{\nu}_{e}$ disappearance results from very SBL reactor experiments such as Bugey, Rovno, Gosgen, ILL, Krasnoyarsk and SRP 38, 39] and the $\stackrel{(-)}{\nu}) \stackrel{(-)}{\nu}$ appearance results in 
LSND [21] and KARMEN [40]. The reactor-based $\bar{\nu}_{e}$ disappearance results do show some results consistent with $\Delta m^{2} \approx 1 \mathrm{eV}^{2}$, however the mixing angles required by these results when considered simultaneously with the $\stackrel{(-)}{\nu_{\mu}} \rightarrow \stackrel{(-)}{\nu}$ appearance results are far too large to be consistent with the $\nu_{\mu}$ disappearance null results. The $\nu_{\mu}$ disappearance results are in direct conflict with the MiniBooNE and other SBL signals under the sterile neutrino interpretation. The primary cause of this conflict is the MiniBooNE LEE, which requires large mixing angles to have been produced by sterile-driven $\stackrel{(-)}{\nu_{\mu}} \rightarrow \stackrel{(-)}{\nu}$ oscillations. This leaves the sterile neutrino interpretation of the LEE heavily disfavoured. 


\section{The MicroBooNE Experiment}

The MicroBooNE experiment is a SBL experiment whose detector is a LArTPC situated $470 \mathrm{~m}$ along the Booster Neutrino Beam (BNB) at Fermilab, the same neutrino beam that was used by MiniBooNE. The primary physics goals of MicroBooNE are to perform measurements of low energy neutrino cross-sections on argon and to determine the source of the MiniBooNE LEE. MicroBooNE's use of the same neutrino beam and similar baseline as MiniBooNE make it well positioned to test different interpretations of the LEE. This chapter includes a description of the BNB as well as the MicroBooNE detector's design, the signatures different types of particles in the detector and how those signatures are observed.

\subsection{Neutrino Flux at MicroBooNE}

The BNB is the source of accelerator produced neutrinos used by both MiniBooNE and MicroBooNE. The BNB can run in neutrino or anti-neutrino mode, producing a beam of primarily $\nu_{\mu}$ or $\bar{\nu}_{\mu}$ respectively (with contamination from $\nu_{e}$ or $\bar{\nu}_{e}$ respectively) [11]. The analysis in this thesis only uses neutrino mode data. The main neutrino beam production stages are as follows:

- $\mathrm{H}^{-}$ions from the Fermilab Linear Accelerator (LINAC) are injected into the Fermilab Booster synchrotron.

- The Fermilab Booster strips the electrons of the ions and accelerates the remaining protons to $8 \mathrm{GeV}$ incident on a beryllium target.

- $\pi^{ \pm}, K^{ \pm}$and $K_{L}^{0}$ mesons are produced by the proton-beryllium interactions.

- The charged mesons are redirected by a magnetic focusing horn into a beam towards neutrino detectors on the beamline.

- The mesons decay to form a neutrino beam.

$\mathrm{H}^{-}$ions from the Fermilab LINAC are injected at $400 \mathrm{MeV}$ into the Fermilab Booster synchrotron. Upon injection the ions are stripped of their electrons, leaving the remaining proton to be accelerated up to $8 \mathrm{GeV}$ (kinetic energy). The accelerated protons are grouped into "beam spills" consisting of approximately $4 \times 10^{12}$ protons over a $1.6 \mu$ s time period. These beam spills are directed from the Fermilab Booster to a beryllium target at an average rate of $5 \mathrm{~Hz}$. Beam characteristics 
are measured by various monitoring systems upstream of the target. Two toroids measure the intensity of the beam spills incident on the beryllium target (protons on target, POT). This is used as a measure of the number of neutrino interactions expected for experiments on the BNB line, including MiniBooNE and MicroBooNE. All plots, event numbers and sensitivities presented in this analysis (excluding the data-MC comparison) assume $6.6 \times 10^{20}$ POT of neutrino mode data. Beam position monitors and a multi-wire chamber track the position and width of the beam. Finally a resistive wall monitor measures the intensity and timing of the beam spills. Figure 3.1 shows the POT received by MicroBooNE as a function of time with the orange region highlighting the unblinded MicroBooNE data used for the data-MC comparison described in this thesis.

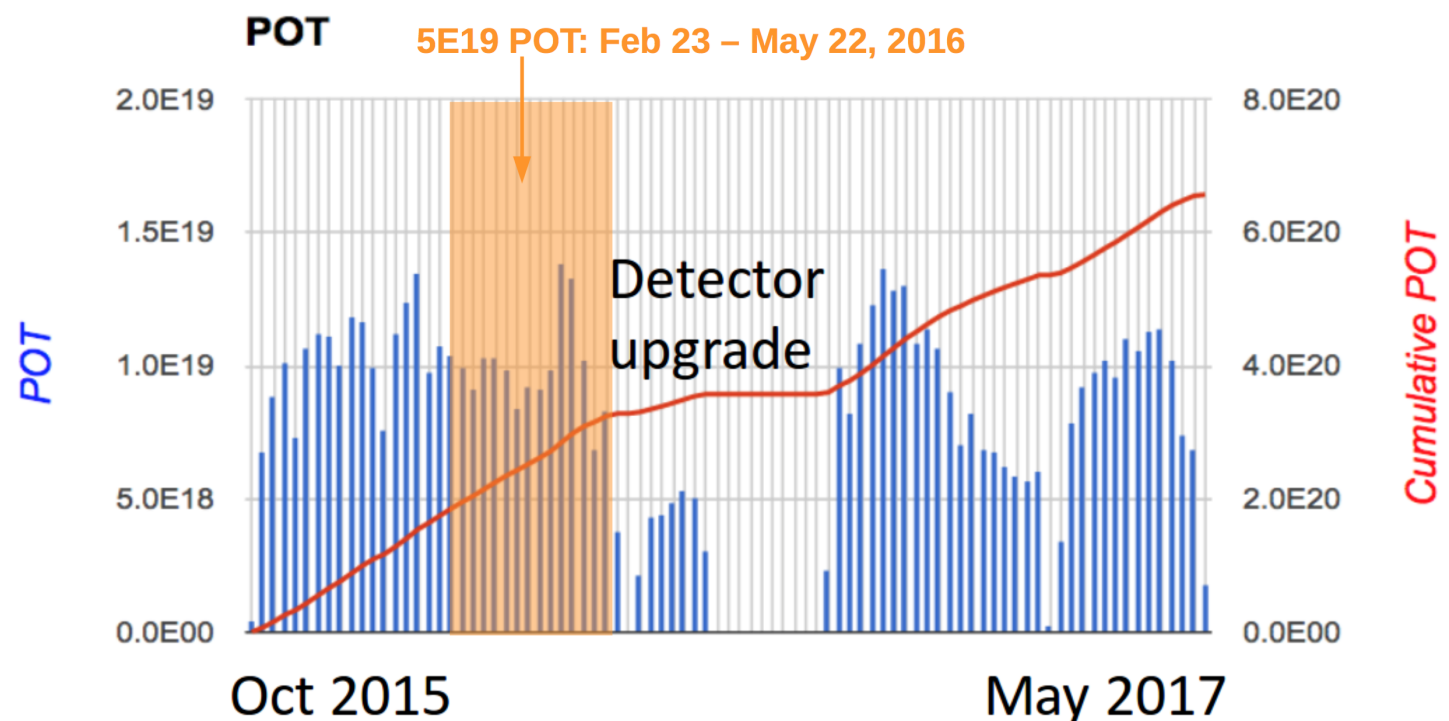

Figure 3.1: Beam stability plot for MicroBooNE showing the POT delivered to MicroBooNE since the beginning of run 1 [41]. The orange region highlights MicroBooNE's currently unblinded data.

The beryllium target consists of seven beryllium slugs arranged into a cylinder $0.51 \mathrm{~cm}$ in radius and $71.1 \mathrm{~cm}$ long. Beryllium is the chosen target material due to its low atomic number which reduces potential radiative energy loss of the protons incident on the target before they collide. The proton-beryllium interactions produce primarily $\pi^{ \pm}$with significant contributions of $K^{ \pm}$and $K_{L}^{0}$ mesons. The beryllium target is situated inside a toroidal magnetic focusing horn comprised of an aluminium alloy. Current with a peak of $170 \mathrm{kA}$ is pulsed through the horn for a duration of $143 \mu$ s coinciding with the arrival of proton beam spills from the Fermilab Booster at the beryllium target. The current pulsed through the horn induces a magnetic field with a peak of $1.5 \mathrm{~T}$ that focuses the produced $\pi^{ \pm}$and $K^{ \pm}$ 
mesons. The direction of the current in the horn can be reversed, in turn reversing the magnetic field. Depending on the direction of the horn current chosen, either the positive or negative mesons the proton-beryllium interactions can be focused (while rejecting mesons of opposite charge), producing a beam of either neutrinos ("neutrino running mode") or anti-neutrinos ("anti-neutrino running mode").

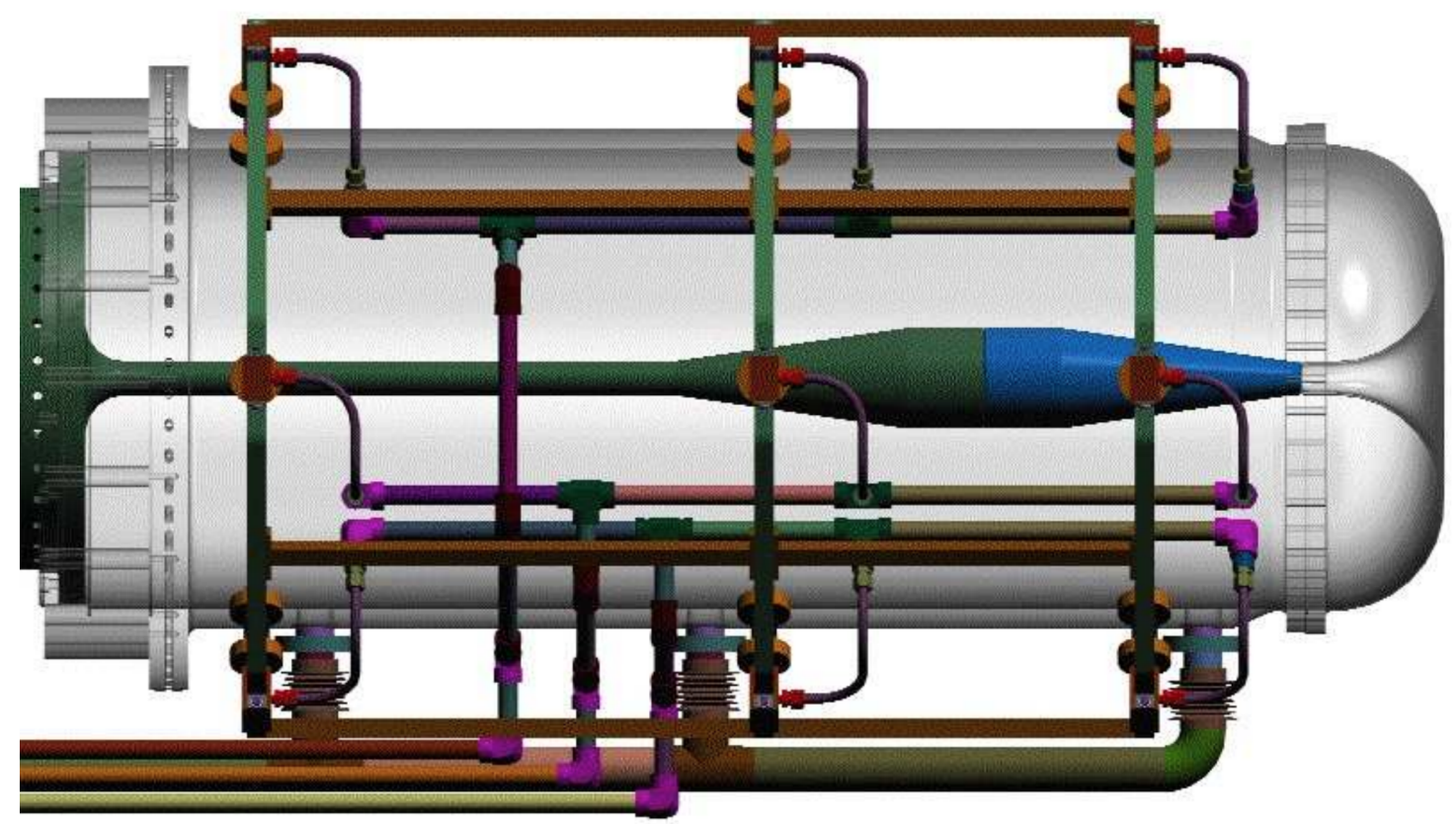

Figure 3.2: The magnetic focusing horn. The beryllium target is located in the central axis of the horn $[11$.

Following the production and horn focusing, particles travel through a $214 \mathrm{~cm}$ long concrete collimator. This collimator absorbs the particles that will not contribute to the neutrino beam, reducing radiation in the beamline. Post collimation, the remaining particles travel down a $50 \mathrm{~m}$ long, $3 \mathrm{ft}$ radius air-filled decay pipe. As they travel down this pipe $\pi^{ \pm}, K^{ \pm}$and $K_{L}^{0}$ will decay, forming a beam-spill of neutrinos. At the end of this decay pipe is a concrete beam stop which absorbs any non-neutrinos remaining post-decay. Figure 3.3 shows the BNB layout including the horn, decay pipe and beam stop.

The neutrinos produced in the decay pipe and that comprise the beam are predominantly $\nu_{\mu}$ with small $\nu_{e}$ contributions (or anti-neutrino counterparts). The vast majority of $\nu_{\mu}$ s in the BNB are produced by $\pi^{+} \rightarrow \mu^{+}+\nu_{\mu}$ decay. The equivalent decay for $\nu_{e}, \pi^{+} \rightarrow e^{+}+\nu_{e}$ is helicity-suppressed by a factor of approximately $10^{4}\left[19\right.$. Helicity-suppression in the $\nu_{e}$ decay mode arises as a consequence of angular momentum conservation and helicity. Consider the decay: $\pi^{+} \rightarrow l^{+}+\nu_{l}$, where $l=e, \mu, \tau$. As charged weak interactions only couple with particles of left-chirality 


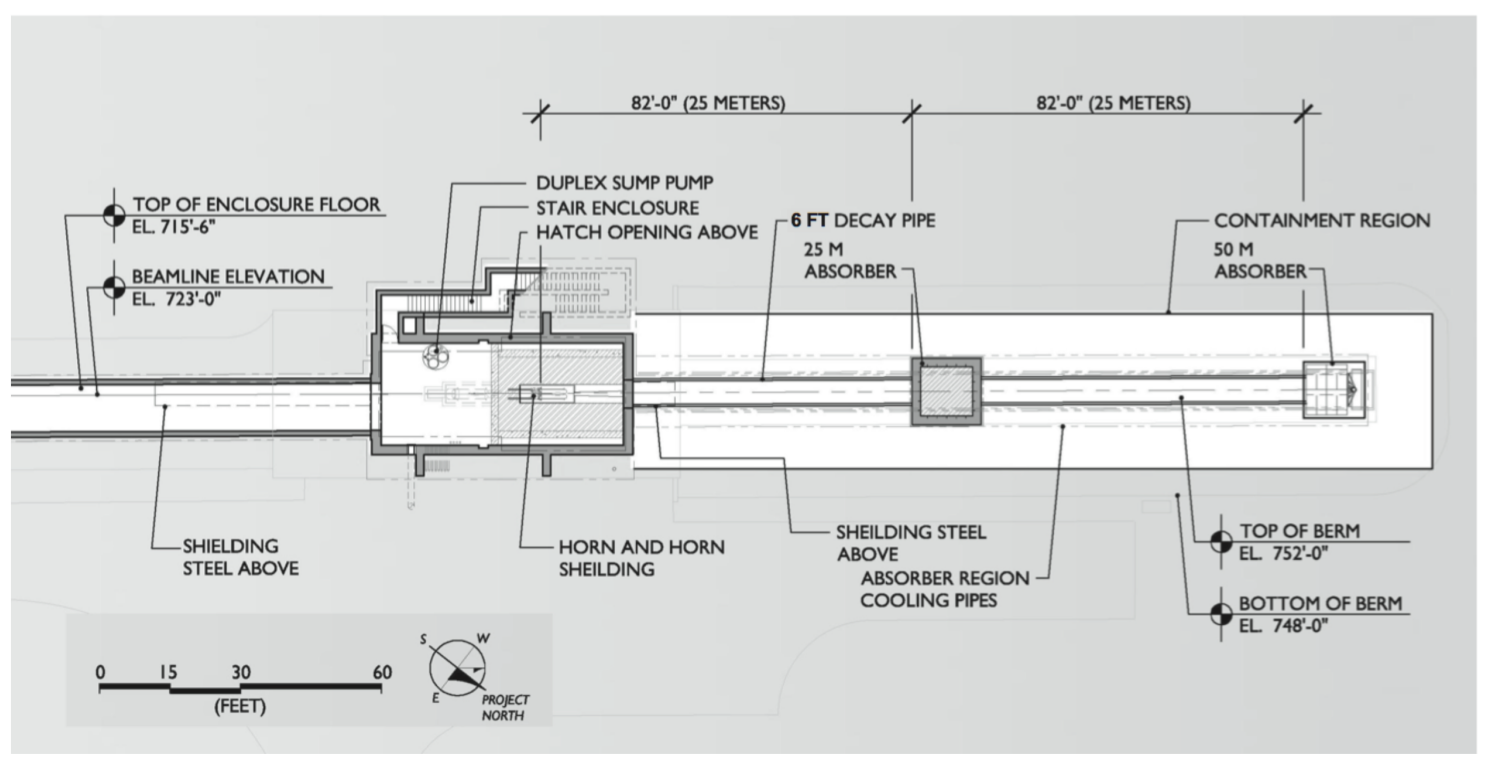

Figure 3.3: Proton beam spills from the Fermilab Booster travel from the left and collide with the beryllium target (located inside the magnetic horn). $\pi^{ \pm}, K^{ \pm}$and $K^{0}$ s are produced at the beryllium target, focused by the horn, and proceed to decay and re-decay into a neutrinos along a $50 \mathrm{~m}$ decay pipe [11].

and anti-particles of right-chirality, the leptons produced by the $\pi^{+}$decay must be left-handed and the anti-leptons right-handed. The helicity of a particle is the projection of that particle's spin on its linear momentum and dictates if the spin of a particle is collinear with its linear momentum (right-helicity) or anti-collinear (lefthelicity). Helicity and chirality are the same in the limit were a lepton is massless. In the $\pi^{+}$decay centre of mass frame the $l^{+}$and $\nu_{l}$ must have equal and opposite linear and angular momentum (as pions are spin 0 particles), requiring both of the produced leptons to have the same helicity. In the massless-lepton limit this decay would not be possible as it would require both lepton and anti-lepton to have the same chirality. For leptons with mass, however, helicity is a linear combination of left and right-handed chiralities. The contribution of the chirality opposite to the helicity of the lepton (e.g. the right-chirality contribution to a left-helicity lepton) is proportional to the mass of that lepton. Neutrinos have such a low mass that they are considered to operate in the massless limit, thus $\nu_{l}$ always has left-chirality and left-helicity. Charged leptons have much higher mass, allowing for the production of a left-helicity and right-chirality $l^{+}$. The dependence of the $l^{+}$production on mass in this way is the reason the $\pi^{+} \rightarrow e^{+}+\nu_{e}$ decay mode is helicity-suppressed relative to the $\pi^{+} \rightarrow \mu^{+}+\nu_{\mu}$ decay mode.

Figure 3.4 shows the simulated flux prediction in MicroBooNE for each neutrino type in terms of energy in neutrino running mode. The majority of neutrino flux arriving at MicroBooNE is $\nu_{\mu}$ produced by $\pi^{+} \rightarrow \mu^{+}+\nu_{\mu}$ however there are sig- 
nificant contributions from $K^{+} \rightarrow \mu^{+}+\nu_{\mu}$ at higher energies. The $\pi^{+}$component of the $\nu_{\mu}$ distribution peaks at approximately $1 \mathrm{GeV}$ with the flatter $K^{+}$distribution becoming dominant at approximately $2.5 \mathrm{GeV}$. The next most significant flux is $\bar{\nu}_{\mu}$ produced primarily by $\pi^{-} \rightarrow \mu^{-}+\bar{\nu}_{\mu}$ which is dominant at all energies. The $\bar{\nu}_{\mu}$ flux is much smaller than the $\nu_{\mu}$ flux due to the magnetic de-focusing of $\pi^{-} \mathrm{s}$ in neutrino running mode. Unlike the $\nu_{\mu}$ flux, the $\bar{\nu}_{\mu}$ flux has no significant contribution from charged $K_{\mathrm{S}}\left(K^{-}\right)$due to a very low production probability in proton-beryllium interactions and magnetic de-focusing. The $\nu_{e}$ flux is produced primarily via $\pi^{+} \rightarrow \mu^{+}+\nu_{\mu}$ followed by $\mu^{+} \rightarrow e^{+}+\nu_{e}$ and $K^{+} \rightarrow \pi^{0}+e^{+}+\nu_{e}$. $\pi^{+}$decay is dominant at $<1 \mathrm{GeV}$ energies and $K^{+}$decay dominates at higher energies. The $\bar{\nu}_{e}$ flux is produced primarily by $K_{L}^{0} \rightarrow \pi^{+}+e^{-}+\bar{\nu}_{e}$ decay. There is no significant contribution from $\pi^{-}$and $\mu^{-}$decay and three-body $K^{-}$decay as these particles are filtered out by magnetic defocusing.

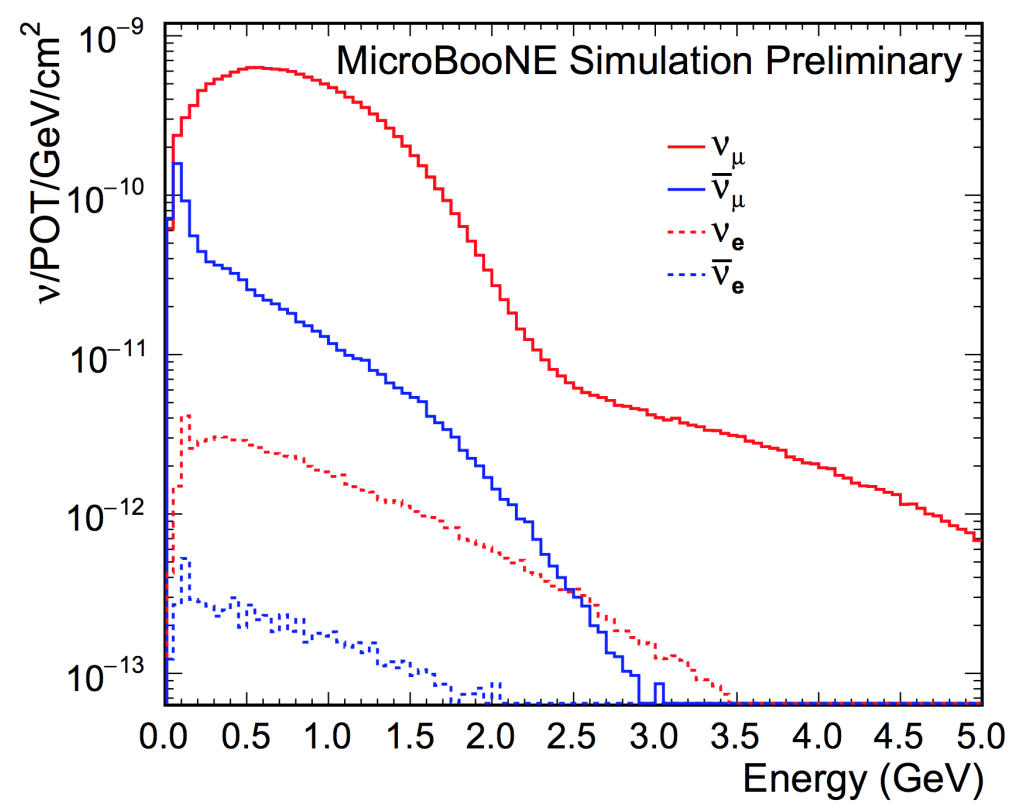

Figure 3.4: Simulated BNB neutrino flux prediction in MicroBooNE for $\nu_{\mu}, \bar{\nu}_{\mu}, \nu_{e}$ and $\bar{\nu}_{e}$ (neutrino running mode) [42].

Systematic uncertainties for the expected BNB neutrino flux in MicroBooNE arise from various sources such as: the measurement of the number of protons delivered to the beryllium target, the production of particles by proton-beryllium interactions, hadronic interactions in the beryllium target and the magnetic focusing horn, the magnetic field produced by the horn and misalignments of beam components relative to their expected positions. Table 3.1 shows the systematic uncertainties for the integrated BNB neutrino flux in MicroBooNE [42]. Uncertainty on protonberyllium particle production is the dominant source of uncertainty for the flux. In all cases the particle with the largest uncertainty contribution for each neutrino type 
listed in Table 3.1 is the particle whose decay is the primary source of that neutrino type in the BNB.

\begin{tabular}{|l|c|c|c|c|}
\hline Systematic & $\nu_{\mu} \%$ & $\bar{\nu}_{\mu} \%$ & $\nu_{e} \%$ & $\bar{\nu}_{e} \%$ \\
\hline Proton delivery & 2.0 & 2.0 & 2.0 & 2.0 \\
$\pi^{+}$ & 11.7 & 1.0 & 10.7 & 0.03 \\
$\pi^{-}$ & 0.0 & 11.6 & 0.0 & 3.0 \\
$K^{+}$ & 0.2 & 0.1 & 2.0 & 0.1 \\
$K^{-}$ & 0.0 & 0.4 & 0.0 & 3.0 \\
$K_{L}^{0}$ & 0.0 & 0.3 & 2.3 & 21.4 \\
Other & 3.9 & 6.6 & 3.2 & 5.3 \\
Total & 12.5 & 13.5 & 11.7 & 22.6 \\
\hline
\end{tabular}

Table 3.1: Systematic uncertainties for the integrated BNB flux for each expected neutrino type in MicroBooNE [42]. The uncertainties on proton delivery and particle production of the most relevant particles are listed. The "other" category contains the combined uncertainties of hadronic interactions, the magnetic field and the misalignment of beam components.

\subsection{Detector}

\subsubsection{Liquid Argon Time Projection Chamber}

A time projection chamber (TPC) consists of a target interaction material, a cathode, and an anode with a read-out system. Energetic particles that are produced in and traverse the target material will ionise the target material atoms they come into close proximity with, ionising them and producing free electrons. The cathode is held at high potential, producing uniform electric field that drifts the ionisation electrons toward the anode where they are detected. The target material must be inert and have high purity for the ionisation electrons to be able to drift through the target material unimpeded.

The MicroBooNE detector is a LArTPC which is contained in a cylindrical cryostat with approximately 86 metric tons of visible (to the detector) liquid argon. Liquid argon is the chosen target material as it is inert and has a relatively high density and neutrino cross-section, increasing the likelihood of neutrino interactions inside the TPC. The detector is located approximately $470 \mathrm{~m}$ downstream of the $\mathrm{BNB}$ and is situated relatively close to ground level. Close proximity to ground level means a large numbers of cosmic particles entering the detector. In MicroBooNE there is an estimated 11,000 cosmic muons traveling through the TPC per second [43]. The maximum drift time for ionisation electrons in MicroBooNE (i.e. 


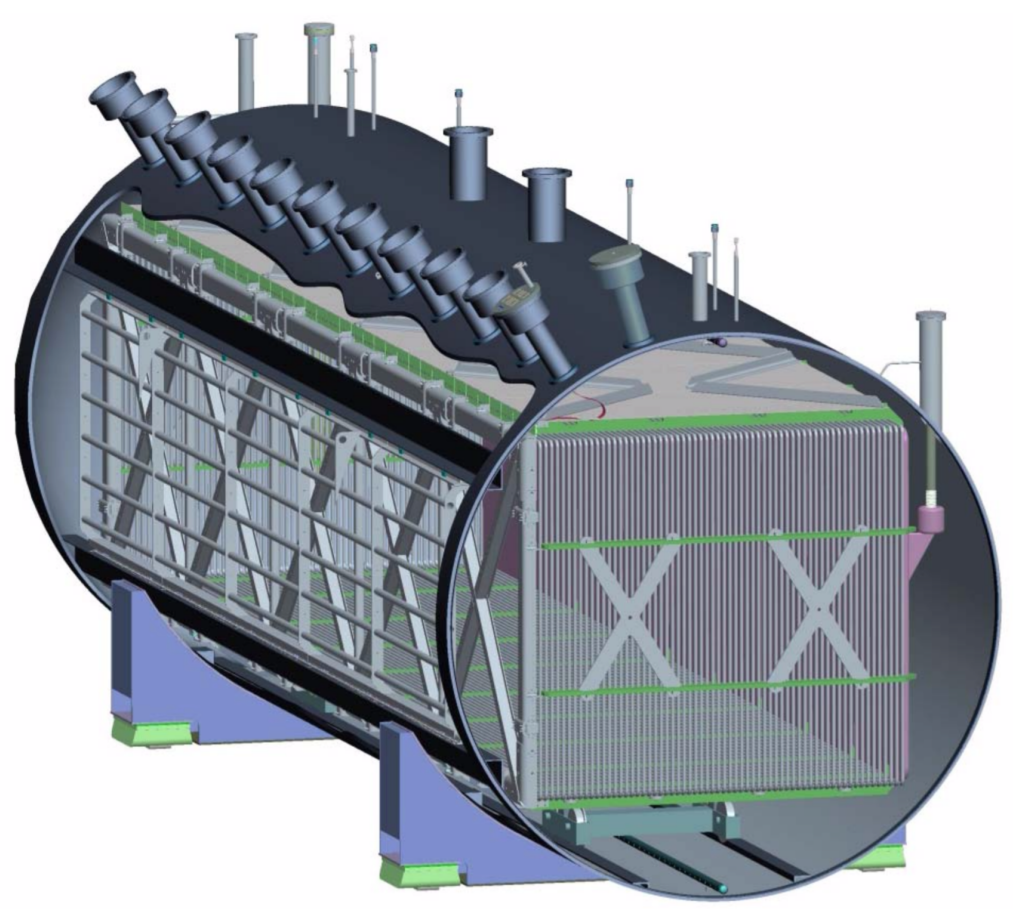

Figure 3.5: A 3-d model of the MicroBooNE detector [43]. The surrounding cylinder is the cryostat which contains the liquid argon and maintains its temperature. Feedthroughs at the top of the cryostat carry wire and PMT information out of the detector. Contained in the cryostat is the cuboid TPC. The three wire planes and the PMTs are located on the left side of the TPC. The field-producing cathode is located on the right side. The remaining four sides comprise the field cage.

the maximum time required for an electron to reach the anode) is approximately 2.2 ms [44] and over this time window roughly $11,000 \times 2.2 \times 10^{-3} \approx 24$ cosmic muons will travel through the TPC. This creates a sizeable background to any BNB based analysis. A 3-d model of the MicroBooNE detector can be seen in Figure 3.5 .

The MicroBooNE TPC is a $2.5 \mathrm{~m} \times 2.3 \mathrm{~m} \times 10.4 \mathrm{~m}$ rectangular cuboid with 9 stainless steel sheets on one side acting as a cathode. This cathode produces a 273 $\mathrm{V} / \mathrm{cm}$ electric field which is made uniform by 64 stainless steel bars surrounding the TPC acting as a field cage. Opposite the cathode is the anode in the form of three planes of ionisation charge sensor wires. The first two wire planes, $\mathrm{U}$ and $\mathrm{V}$, are induction planes consisting of 2400 wires each, orientated at \pm 60 degrees to vertical. The third plane is a vertical collection plane consisting of 3456 wires. The inter-wire spacing in each plane and inter-plane spacing is $3 \mathrm{~mm}$. Behind the wire planes is an array of 32 photomultiplier tubes (PMTs) that are used to detect scintillation light produced by neutrino interactions and charged particles as they traverse the liquid argon 43]. 


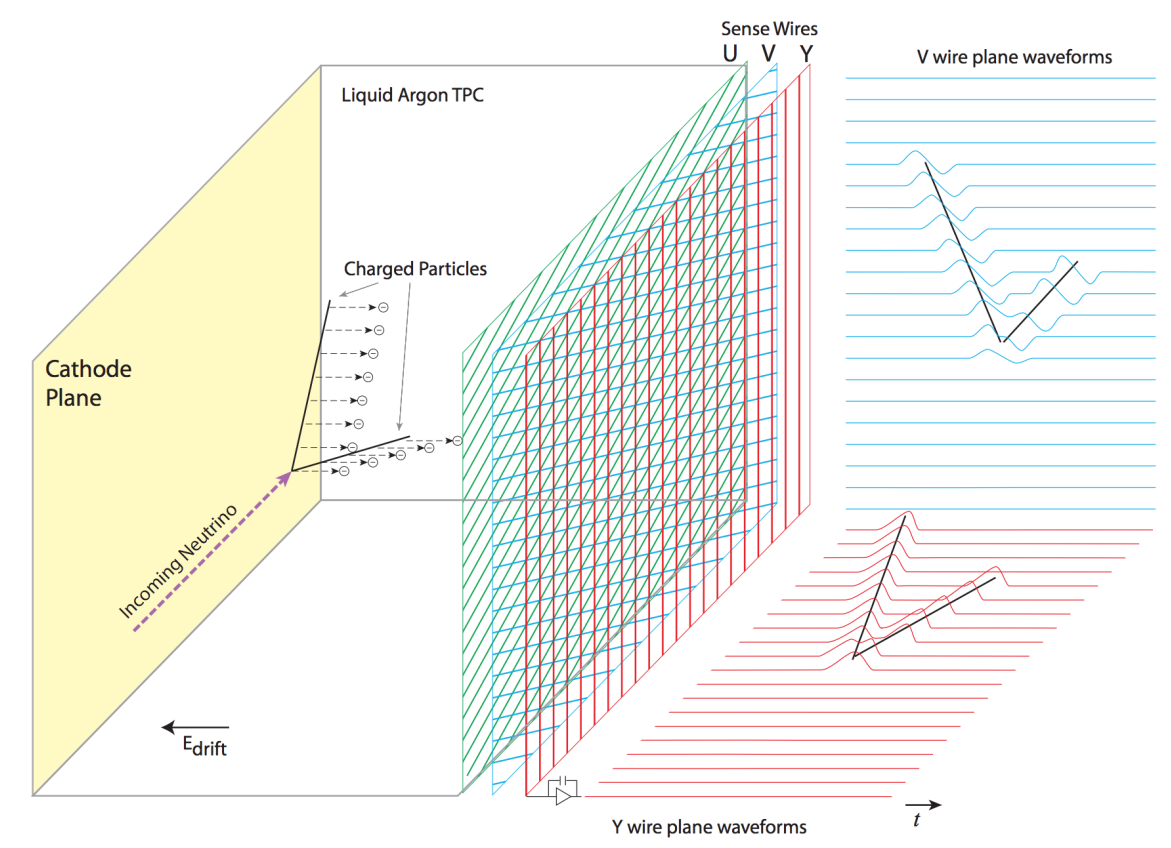

Figure 3.6: A diagram showing the detection of charged particles in a LArTPC [43]. A neutrino enters the LArTPC and produces charged particles which travel through and ionise the liquid argon. The ionisation electrons from these charged particles are then drifted towards the three wire planes: $\mathrm{U}, \mathrm{V}$ and the colection plane ( $\mathrm{Y}$ in the diagram) by a uniform electric field. The ionisation electrons produce a signal in the wire planes upon reaching them.

\subsubsection{Operating Principles}

A particle traversing the liquid argon will produce ionisation electrons which are drifted from the cathode to the wire planes by the uniform electric field as shown in Figure 3.6. Electrons drifting past the first two wire planes (induction planes $U$ and $\mathrm{V})$ will induce a bipolar signal in these planes before being collected and inducing a unipolar signal in the collection plane (Y). A $-124 \mathrm{~V}$ bias is applied to the first induction plane $(\mathrm{U})$ and a $186 \mathrm{~V}$ bias is applied to the collection plane to ensure ionisation electrons reach it (the middle induction plane, $\mathrm{V}$, is held at ground) 45]. Each wire plane shields the proceeding wire plane meaning ionisation electrons will only induce a signal in a wire plane once they have passed the preceding wire plane. The $\mathrm{U}$ induction plane has no preceding wire plane and so is much more sensitive to ionisation electrons in the TPC. The induced wire signals are used to reconstruct the 2 -d position of the ionisation electrons on each of the three wire planes.

Position in the $3^{\text {rd }}$ dimension can be determined by using the understood ionisation electron drift velocity in liquid argon $(114 \mathrm{~cm} / \mathrm{ms}$ at an electric field of 273 $\mathrm{V} / \mathrm{cm}$ [4]) with the ionisation electron travel time. Travel time can be determined by assuming the ionising particle was produced by a neutrino interaction from the 
BNB (not the case for cosmic particles) and measuring the time difference between the arrival of BNB neutrinos (known from a signal sent by the BNB as beam spills are sent) and the arrival of ionisation electrons at the wire planes. A more precise travel time can be determined using scintillation light produced produced by charged particles which will reach the PMTs in $\mathcal{O}(10)$ ns 43$]$.

The raw input for the TPC is the signal induced on each of the wire planes by drifting ionisation electrons. Signals induced in the wires are processed via analogue to digital conversion (ADC) with each wire presenting its signal in terms of ADC counts over a range of time "ticks". A tick is a discrete measure of time whose precision is based on the operating frequency of the data acquisition system (DAQ) outputting data. MicroBooNE's DAQ operates at a frequency of $2 \mathrm{MHz}$ [45] making each tick $0.5 \mu \mathrm{s}$ in duration.

The most commonly occurring visible particles in MicroBooNE are minimally ionizing particles (MIPs). These are particles such as muons or charged pions and deposit only a small amount of energy into liquid argon as they travel through it. A typical MIP is expected to have a rate of energy deposition $(\mathrm{dE} / \mathrm{dx})$ of approximately $2.06 \mathrm{MeV} / \mathrm{cm}$ in liquid argon [45]. Drift electrons produced via the ionisation of argon atoms have a chance to combine with the positive argon ions produced in the same ionisation, resulting in stable argon atoms. The loss of ionisation electrons in this manner is called recombination. The number of ionisation electrons deposited on a wire on a given plane, $n_{e}$, by a typical MIP traveling through the TPC is described by

$$
n_{e}=\frac{d E}{d x} \frac{R x}{w_{\mathrm{Ar}}}
$$

where $R$ is the recombination factor (0.62 in MicroBooNE 46]), $x$ is the distance travelled between wires and $w_{\mathrm{Ar}}$ is the work required to ionise an atom of argon (23.6 $\mathrm{eV}[47,48])$. Assuming the MIP is traveling perpendicularly to the wires ( $x=3 \mathrm{~mm})$, $n_{e}=2.06 \times 10^{6} \times \frac{0.62 \times 0.3}{23.6} \approx 1.62 \times 10^{4}$ on each wire the MIP passes. Studies of BNB-produced stopping muons in MicroBooNE [49] have determined a calibration constant for each wire plane that directly relates $n_{e}$ to the number of expected ADC counts produced by the electrons on that wire according to

$$
n_{\mathrm{ADC}} \times C_{p}=n_{e}
$$

where $n_{\mathrm{ADC}}$ is the number of expected $\mathrm{ADC}$ counts on a wire and $C_{p}$ is the calibration constant for a given wire plane. The constant for each plane is listed in Table 3.2 for both data and MC. For a typical data MIP observed by and traveling perpendicularly to the collection plane, $n_{\mathrm{ADC}}=1.62 \times 10^{4} \times 4.12 \times 10^{-3} \approx 67$ on 
each wire the MIP passes.

\begin{tabular}{|l|c|c|}
\hline Wire Plane & MC & Data \\
\hline $\mathrm{U}$ & $5.18 \pm 0.02 \times 10^{-3}$ & $4.23 \pm 0.02 \times 10^{-3}$ \\
$\mathrm{~V}$ & $5.08 \pm 0.02 \times 10^{-3}$ & $4.37 \pm 0.02 \times 10^{-3}$ \\
Collection & $5.08 \pm 0.02 \times 10^{-3}$ & $4.12 \pm 0.02 \times 10^{-3}$ \\
\hline
\end{tabular}

Table 3.2: Calibration constants used to directly relate the number of ionisation electrons on a wire to the number of expected ADC counts. Listed are the constants for each plane, for both data and MC [49].

Effects of the detector itself can alter the TPC analogue wire signal and its subsequent digitised form making the "true" ionisation electron signal more difficult to reconstruct. These effects are mitigated in two stages of software-based signal processing. The first is noise filtering which aims to reduce electronic noise and other irregularities. The second stage is signal deconvolution which aims to reverse signal-altering effects from the detector's electronics and the impact of the drift field on the distribution of ionisation electrons at the wire planes. Following signal processing, "hit" finding is applied to the modified wire signals. Groups of ionisation electrons will usually induce wire signals which are gaussian-distributed. Hit-finding attempts to fit one or more Gaussian distributions to the wire-signal as shown in Figure 3.7. The complexity of the wire signal will dictate the number of gaussians required to accurately represent it. A wire signal that has been successfully fitted with one or more gaussians is referred to as a hit.

Detector information is stored in an "event" when some form of trigger is received by the detector. An event consists of $4.8 \mathrm{~ms}$ of TPC information divided into three $1.6 \mathrm{~ms}$ long "frames". The frames are aligned such that the first frame covers the $1.6 \mathrm{~ms}$ before the arrival of the trigger and the two subsequent frames cover the $3.2 \mathrm{~ms}$ after. The $1.6 \mathrm{~ms}$ frame duration was chosen based on the maximum predicted electron drift time from cathode to anode for an electric field strength of $500 \mathrm{~V} / \mathrm{cm}$ 43] (though MicroBooNE is currently operating at a field strength of $273 \mathrm{~V} / \mathrm{cm}$ resulting in a slightly higher maximum drift time of $2.2 \mathrm{~ms}$ [44]). The purpose of extending the duration of an event to include TPC activity not feasibly from BNB interactions is to allow for the reconstruction of cosmic ray particles that arrived prior to, or after the arrival of the BNB neutrino beam spill in the detector. Also stored in the event is $23.4 \mu$ s of PMT information starting at the arrival time of the trigger, with the expected $1.6 \mu \mathrm{s}$ beam spill (see Section 3.1) exposure time being over the 3.2-4.8 $\mu$ s range (henceforth the beamgate). 


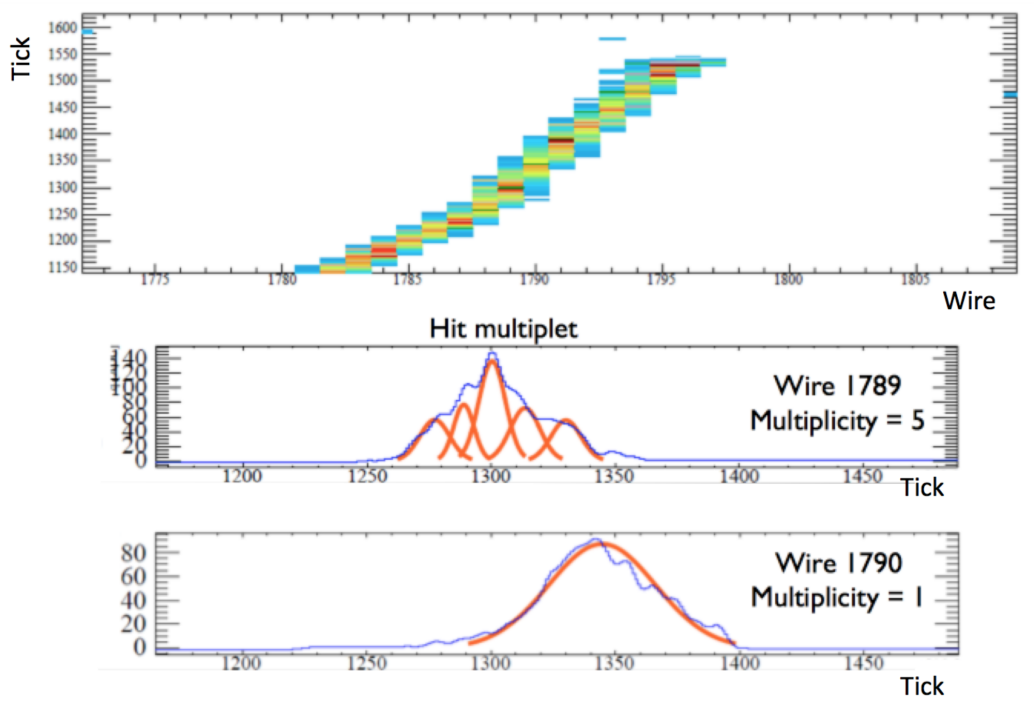

Figure 3.7: A demonstration of the hit-finding process [45]. The top plot shows a simulated low-momentum particle traveling through the TPC and producing ionisation electrons which go on to induce signals on the collection plane. These signals are characterised by attempting to fit one or more gaussians to them. The x-axis is the wire channel, the $\mathrm{y}$-axis is time in discrete ticks $(0.5 \mu \mathrm{s}$ in duration) and the z-axis is the number of ADC counts on the wire at a given time. The middle plot shows a complex signal requiring five Gaussian distributions to accurately describe the signal. The bottom plot shows a simple wire signal where only one gaussian is required. The $\mathrm{x}$ and $\mathrm{y}$ axes of the last two plots are discrete ticks and ADC counts respectively.

Various triggers are used by the MicroBooNE detector to select which information is saved and which is discarded. The triggers relevant to this analysis are the ones used to save BNB neutrino-interactions: the BNB hardware trigger and the beam-gate PMT trigger. The BNB hardware trigger is sent to detectors along the BNB alerting them when a neutrino beam spill is sent. Due to the low interaction cross-sections of neutrinos, the probability that a BNB beam-spill producing a neutrino interaction in the MicroBooNE detector is approximately 1/600 [43]. This means the vast majority of BNB hardware trigger events will contain no neutrino interactions, wasting detector resources to record and potentially adding to the cosmic background of MicroBooNE analyses. The scintillation light produced by neutrino interactions can be used as a powerful tool to determine whether a neutrino interaction took place, as the light will arrive at the PMTs in $\mathcal{O}(10)$ ns, far faster than any ionisation electrons will reach the TPC wires. The beam-gate PMT trigger takes advantage of this by requiring that in a BNB hardware triggered event there is a 100 ns time interval in the $1.6 \mu$ s beam-gate that has a summed PMT pulse-height $\geq 2$ and a PMT multiplicity $\geq 1$ in order to trigger. This indicates an interaction occurred during the beam-spill, increasing the likelihood that event contains a BNB interaction. Events possessing both triggers are recorded for analysis. 


\subsubsection{Particle Signatures}

The average rate of energy loss by ionisation as a charged particle travels through matter is described by the Bethe-Bloch formula [19],

$$
\left\langle-\frac{d E}{d x}\right\rangle=\frac{4 \pi}{m_{e} c^{2}} \frac{n_{e} z^{2}}{\beta^{2}}\left(\frac{e^{2}}{4 \pi \epsilon_{0}}\right)^{2}\left[\ln \left(\frac{2 m_{e} c^{2} \beta^{2}}{I\left(1-\beta^{2}\right)}\right)-\beta^{2}-\frac{\delta}{2}\right],
$$

where $m_{e}$ is the mass of an electron, $c$ is the speed of light, $n_{e}$ is the electron density of the matter the particle is traversing, $z$ is the charge of the traversing particle, $\beta=\frac{v}{c}$ where $v$ is the speed of the particle, $\epsilon_{0}$ is the permittivity of free space, $I$ is the mean excitation energy of the matter and $\delta$ is a matter density correction term describing polarisation of the matter by the traversing particle.

There are two different topological signatures that can be imprinted by a particle in the TPC depending on the particle's type. The first is a "track" which is a signature predominantly left by MIPs. At the energies expected in MicroBooNE, particles such as these typically reside in an energy region which is high enough for energy loss via ionisation effects (as described by equation 3.3) to be minimal but low enough that energy loss via radiative effects insignificant. MIPs typically travel in a straight line through through the TPC. As they travel they will ionise the argon in their path producing ionisation electrons. This resulting line of ionisation electrons will drift to the wire planes where they will be reconstructed by the detector into a line of hits on each wire plane.

The second signature imprinted by a particle in the TPC is an "electromagnetic shower" (shower for short) and is a special case that is only produced by electrons, positrons and photons in MicroBooNE. In terms of electromagnetic shower production electrons and positrons behave in a very similar way, henceforth mention of an electron can be taken to mean an electron or positron unless a positron is explicitly mentioned. Due to their low mass relative to the other types of charged particle typically produced in the TPC (i.e. track producing MIPs), electrons produced at typical MicroBooNE energies reside in the energy region where radiative energy loss effects are prevalent. Figure 3.8 shows the stopping power of liquid argon for electrons as a function of energy. The dominant form of radiative energy loss in electrons is that of bremsstrahlung photon production. This is a process by which an electron comes into close proximity with and is subsequently decelerated by an argon atom. This deceleration causes the electron to lose energy which results in 
the production of a photon (of energy equal to the energy lost by the electron).

Liquid Argon Stopping Power for Electrons

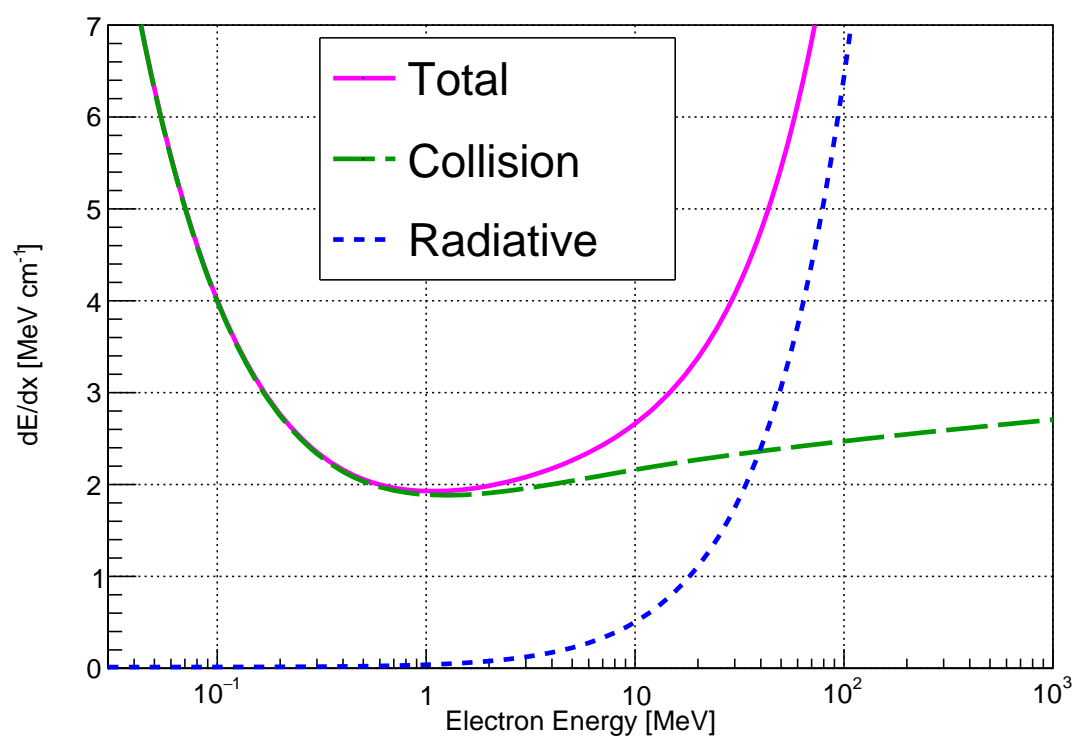

Figure 3.8: Stopping power of liquid argon for electrons in terms of electron energy made using data from NIST ESTAR [50]. Shown is the total stopping power and the collision and radiative components.

An electron can repeatedly produce bremsstrahlung photons as long as it has sufficient energy. Photons of sufficient energy produced in the TPC (such as bremsstrahlung photons) will interact with argon nuclei to produce an electronpositron pair (via pair production). The electron and positron in this pair (assuming they have sufficient energy) will both go on to produce more bremsstrahlung photons. The combination of bremsstrahlung and pair production results in a chain reaction of repeated particle production which continues until the bremsstrahlung photons no longer possess the energy to pair produce and the pair-produced electrons and positrons no longer posses the energy to produce more bremsstrahlung photons. Photons, electrons and positrons are part of every shower but only the electrons and positrons are able to ionise argon atoms (as they are charged particles) and therefore the only parts of the shower that is visible to the TPC.

The primary particle of a shower can also be a photon. The main difference between a photon shower and an electron shower is that a photon shower will start with a photon pair producing whereas an electron shower will start immediately start ionising and subsequently produce photons through bremsstrahlung radiation. As can be seen in Figure 3.8, when an electron is not producing bremsstrahlung photons (i.e. when the radiative component of the stopping power is ignored and 
only the collision component is considered), its $\mathrm{dE} / \mathrm{dx}$ is a low and relatively constant $2-2.7 \mathrm{MeV} / \mathrm{cm}$, that of a MIP. This MIP behaviour is extremely valuable as it allows for the differentiation between photon and electron showers by studying the MIP particle traces at the beginning of each shower. The difference in initial shower production results in differing $\mathrm{dE} / \mathrm{dx}$ values for the start of photon and electron showers, with photon showers having two MIPs (an electron and a positron) at the beginning of the shower and thus approximately double the $\mathrm{dE} / \mathrm{dx}$ of an electron shower which has only one MIP at the start (the electron itself).

Figure 3.9 shows $\mathrm{dE} / \mathrm{dx}$ values for the beginning of simulated photon and electron showers. For electron showers this is the $\mathrm{dE} / \mathrm{dx}$ of the initial electron from the point of production to the point at which it first produces bremsstrahlung photons. For photon showers this is the combined $\mathrm{dE} / \mathrm{dx}$ of the initial electron-positron pair, measured from the pair-production of both particles to the point at which they first produce bremsstrahlung photons. As expected the $\mathrm{dE} / \mathrm{dx}$ distribution for the simulated electron showers peaks at approximately $2 \mathrm{MeV} / \mathrm{cm}$, while the distribution for the simulated photon showers peaks at approximately $4 \mathrm{MeV} / \mathrm{cm}$, double that of the electron showers. Henceforth any reference to the " $\mathrm{dE} / \mathrm{dx}$ " of a shower will refer to the " $\mathrm{dE} / \mathrm{dx}$ " at the start of the shower that can be used to identify the shower as having come from a photon or electron.

Electrons will begin to ionise the liquid argon in MicroBooNE almost immediately after they are produced, thus the start of an electron shower will be in the same position as the vertex of the interaction that produced it. Photons do not ionise liquid argon and thus the start of a photon shower is the point of pair production. The distance travelled before a photon pair produces can be characterised in terms of a photon's "radiation length". The radiation length is $7 / 9$ of the mean free path for high energy photon pair production [51] and can be determined for a given material using

$$
\frac{1}{X_{0}}=4 \alpha r_{e}^{2} \frac{N_{A}}{A}\left\{Z^{2}\left[L_{r a d}-f(Z)\right]+Z L_{r a d}^{\prime}\right\},
$$

where $X_{0}$ is the material-density-independent radiation length $\left(\mathrm{g} \mathrm{cm}^{-2}\right), \alpha$ is the fine-structure constant, $r_{e}$ is the classical electron radius, $N_{A}$ is Avogadro's number, $A$ is the molar mass of the material, $Z$ is the atomic number of the material, $L_{r a d}, L_{\text {rad }}^{\prime}$ and $f(Z)$ are material-specific variables [52]. For argon: $A=39.948 \mathrm{~g} \mathrm{~mol}^{-1}, Z=18, L_{r a d}=4.252, L_{\text {rad }}^{\prime}=5.158$ and $f(Z)=0.0204$ giving a value of $X_{0}=19.549 \mathrm{~g} \mathrm{~cm}^{-2}$. For a liquid argon density of $1.3954 \mathrm{~g} \mathrm{~cm}^{-3}$ the radiation length of a photon is $X_{0} / 1.3954 \approx 14.01 \mathrm{~cm}$. The values for the molar mass of argon 


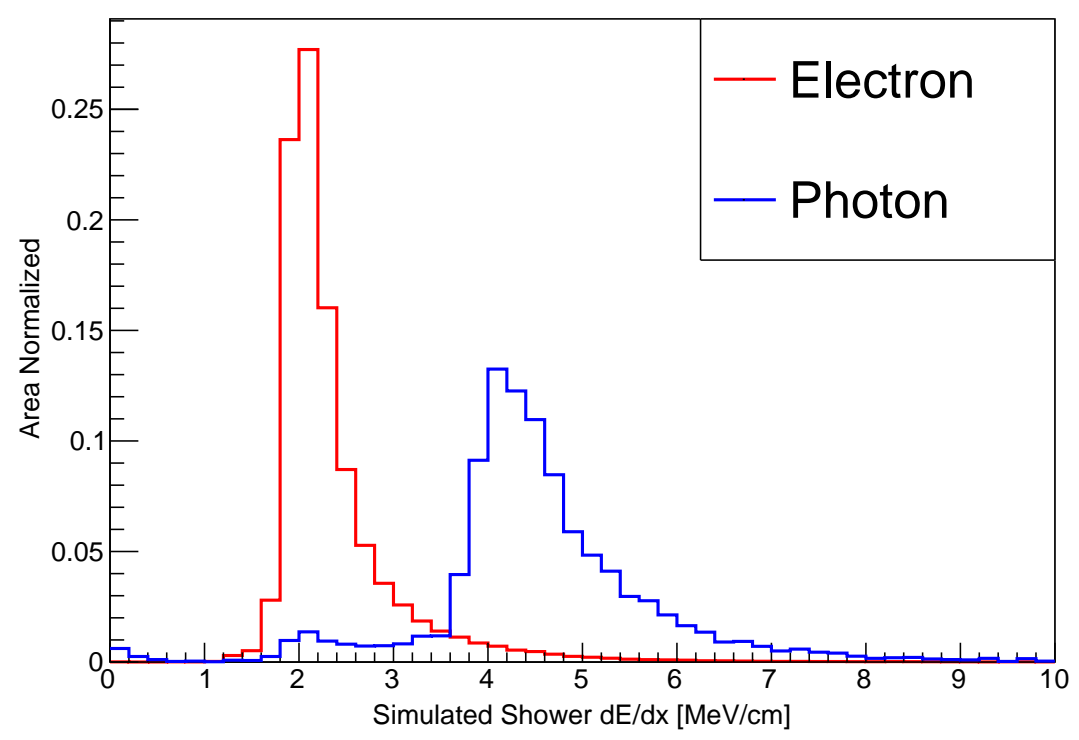

Figure 3.9: True $\mathrm{dE} / \mathrm{dx}$ for the beginning of simulated photon and electron showers in MicroBooNE. For electron showers this is the $\mathrm{dE} / \mathrm{dx}$ of the initial electron from the point of production to the point at which it first produces bremsstrahlung photons. For photon showers this is the combined $\mathrm{dE} / \mathrm{dx}$ of the initial electron-positron pair, measured from the pair-production of both particles to the point at which they first produce bremsstrahlung photons.

and the density of liquid argon were taken from Ref [19]. The $\mathrm{dE} / \mathrm{dx}$ differences between photon and electron showers and the $14 \mathrm{~cm}$ photon shower start-interaction vertex gap in MicroBooNE make it an ideal experiment to determine the identity of the showers it observes and thus to identify the MiniBooNE LEE as photon or electron in nature.

\subsubsection{Systematic Uncertainties}

Potential contributions to systematic uncertainties from detector modeling include uncertainties on:

- The amount of scintillation light produced by particles in the TPC.

- Liquid argon purity. Affects the ionisation electron drift time and the amount of light observed by the PMTs.

- PMT noise.

- Ionisation electron lifetime. Affects the likelihood of ionisation electrons reaching the wire planes. 
- Recombination effects. Electrons produced by the ionisation of argon atoms can potentially recombine with the resulting argon ions, leading to a reduction in the charge observed by the wire planes.

- Drift field uniformity. Irregularities in the electron drift field can affect the distribution of charge that reaches the wire planes in different parts of the detector.

- Space charge effects. Production of ionisation electrons also results in the production of slow moving positive ions. Build-up of such positive charge can result in irregularities in the local drift field.

- Transverse and longitudinal diffusion of drifting electron charge.

- Induced wire charge. Affects the distance at which the wires can "see" ionisation electrons in the TPC.

- Wire noise.

Initial estimates of detector systematics in the SBN proposal [53] indicated that the contribution of detector systematics to overall BNB analysis uncertainty would be small relative to other contributions such as flux and cross-section. These estimates were made under the assumption of a full working knowledge of LArTPCs. The initial estimates of detector systematics from MicroBooNE analyses are much larger, placing the total detector systematic uncertainty at approximately $20 \%$ [54,55]. The most significant contributions to this uncertainty being from the induced wire charge at $15 \%$ and wire noise at $6.4 \%$ [54]. These are conservative estimates and should decrease as the understanding of LArTPCs matures. i 


\section{Event Simulation and Reconstruction}

\subsection{Monte Carlo Event Simulation}

Event simulation in MicroBooNE begins with a Monte Carlo (MC) event generator. BNB neutrino events are generated using the GENIE (Generates Events for Neutrino Interaction Experiments) generator (v2.12.2) [56]. GENIE generates BNB neutrino-argon interactions (interactions with argon nuclei and orbiting electrons) over a specified volume in the MicroBooNE detector. This volume is typically either the volume of the entire cryostat or the volume of the TPC (the volume in which particles are visible to the detector). In order to generate neutrino events, GENIE requires information regarding the expected flux of neutrinos at the detector. This flux is determined by simulating the BNB in Geant4 (GEometry ANd Tracking) 57 (v4.10.1). The simulation of the BNB includes the initial proton-beryllium target interaction, resulting production of $\pi^{ \pm}, K^{ \pm}$and $K_{L}^{0}$ mesons, focusing by the BNB horn and finally particle decay and re-decay into neutrinos. Information from the resulting neutrinos is used to create a set of neutrino flux files that can be used by GENIE to predict the expected BNB neutrino flux at MicroBooNE (see Figure 3.4).

The physics models used by GENIE to generate neutrino interactions include the dominant scattering mechanisms over the $\mathcal{O}(1 \mathrm{MeV}-100 \mathrm{GeV})$ energy range and are usable for any neutrino and neutrino-target type. These models include the nuclear physics model, cross-section model and hadronization model. GENIE uses the Bodek and Ritchie relativistic Fermi gas model [58], which is a nuclear physics model applicable over a large range of energies and targets, for its nuclear physics model. The cross-section model uses the total cross-sections of incoming neutrinos in conjunction with neutrino flux to determine the energy of the neutrinos. Crosssections for specific interactions are then used to determine what kind of neutrino interaction occurs and the interaction model is subsequently used to determine event kinematics. These neutrino interactions include:

- Quasi-Elastic Scattering: Scattering in which energy transfers are small relative to the incident particles. Calculated using the Llewellyn-Smith model [59].

- NC Elastic Scattering: Calculated using the model described in Ref [60]. 
- Baryon Resonance Production: Production of higher-energy baryon resonances such as the $\Delta$ resonance. Calculated using the Rein-Sehgal model [61] which in turn uses the Feynman-Kislinger-Ravndal model of baryon resonances 62].

- Coherent Pion Production: Production of predominantly forward-going pions, calculated using the Rein-Sehgal model.

- Inelastic and Deep Inelastic Scattering: Higher energy scattering that does not conserve the energy of the incident particle. Calculated in an effective leading order model using modifications from Bodek and Yang [63].

- Inverse muon decay: calculated using the Bardin and Dokuchaeva model [64].

- Neutrino-electron elastic scattering: All neutrino-electron scattering, excluding inverse muon decay, is calculated using Ref [65].

The hadronization model determines final state particles, particle four-momenta and the kinematics of the event based on the type of neutrino interaction that took place. GENIE uses the AKGY hadronization model which combines two separate models. The choice of model is dependent on the invariant mass of the interaction. Interactions in the lower invariant mass region use a phenomenological description described by Koba-Nielsen-Olesen (KNO) scaling [66]. As the invariant mass of interactions increases, the model is gradually switched to the PYTHIA/JETSET model [67]. The transition from KNO to PYTHIA/JETSET is performed gradually over an intermediate invariant mass range. As invariant mass in this range increases, the fraction of interactions modeled using PYTHIA/JETSET is linearly increased from $0 \%$ to $100 \%$.

The output of GENIE neutrino event generation is a set of final state particles produced by the neutrino interaction after final state interactions in the nucleus have taken place (including the outgoing neutrino and remaining argon/nuclear fragment). Cosmic particles in MicroBooNE are generated using the CORSIKA generator [68] which generates a set of downward-facing cosmic particles, $18 \mathrm{~m}$ above the center of the TPC. The particles produced by one or both of the GENIE and CORSIKA generators are handed to Geant4 which propagates the particles through the liquid argon, simulating particle decays, interaction, re-interactions and energy deposition in the liquid argon based on the initial states of the particles received. 


\subsubsection{NC $\Delta$ Radiative Modeling}

While there has been no measurement of neutrino-induced NC single photon production, there are several models that provide cross-section predictions for the process. Figure 4.1 shows a selection of these cross-section predictions including neutrino event generators such as GENIE [56], NEUT [69] (used by T2K) and NUANCE [70] (used by MiniBooNE). Mostly good agreement is seen between the models at lower energies (the energies relevant to the LEE), however the models do diverge as energy increases. These cross-section predictions are for inclusive neutrino-induced $\mathrm{NC}$ single photon production, not just NC $\Delta$ radiative decay (though it does account for the vast majority at lower energies [71]). As this thesis considers only NC $\Delta$ radiative decay as signal, additional NC single photon producing processes such as anomaly mediated photon production and generalized Compton scattering will contribute to background 72 . Such processes are relatively rare compared to other photon producing processes in MicroBooNE ( $\operatorname{such}$ as $\pi^{0} \rightarrow 2 \gamma$ ) and so are not likely to have a significant impact on the analysis.

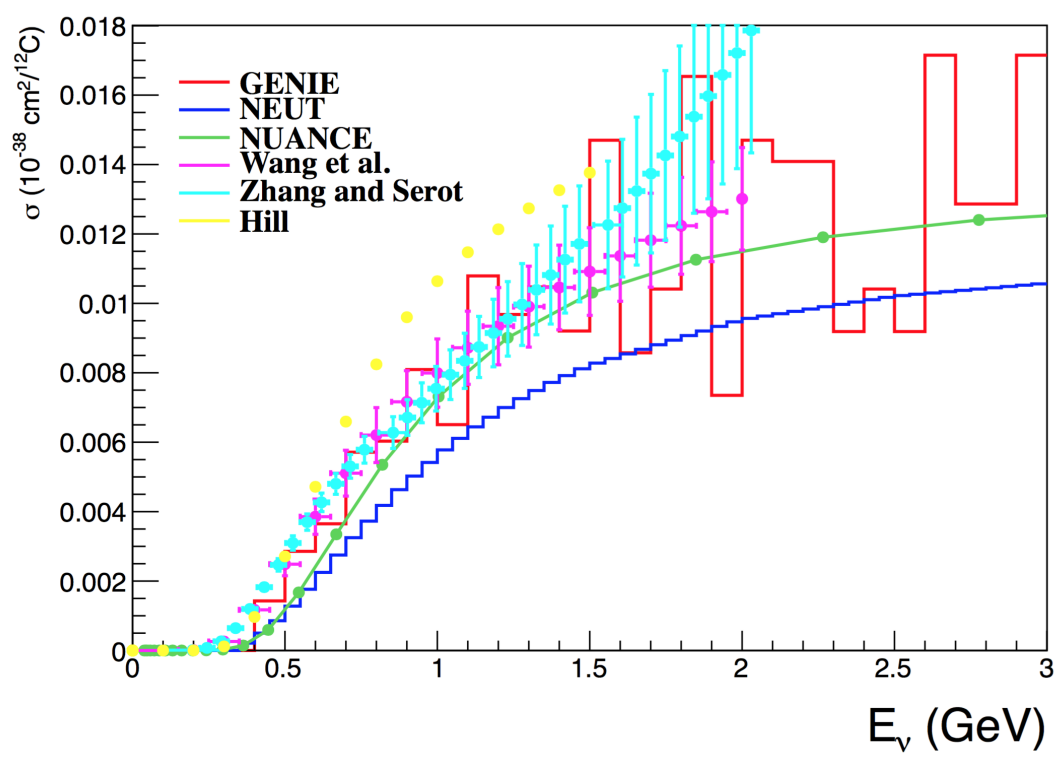

Figure 4.1: A comparison of neutrino-induced NC single photon cross-section models by T. Katori [73]. The cross-sections determined by each model are for carbon and are plotted in terms of true neutrino energy. Included are the GENIE, NEUT and NUANCE neutrino event generators as well as theoretical predictions from Hill [72], Wang et al. [74] and Zhang and Serot [75].

Of particular interest is the comparison between GENIE and NUANCE as these are the neutrino event generators used by MicroBooNE and MiniBooNE respectively. The NUANCE implementation of NC $\Delta$ radiative decay was based on the in-situ 
NC $\pi^{0}$ measurement made by MiniBooNE rather than on a theoretical model. This implementation used the NC $\Delta$ radiative branching ratio (approximately $0.6 \%$, see Section 2.3.1 to determine the ratio of $\Delta$ produced $\gamma$ to $\pi^{0}$ on carbon. This ratio was then scaled by the NC $\pi^{0}$ measurement to determine the expected NC $\Delta$ radiative rate. Given the subject of this thesis is to search for a NC $\Delta$ radiative LEE in MicroBooNE, agreement between the NUANCE and GENIE implementations of NC $\Delta$ radiative decay is crucial. Figure 4.1 shows GENIE is in good agreement with NUANCE and thus is a viable option for the analysis.

The modeling of neutrino-induced NC resonant $\Delta$ production and subsequent radiative decay in MicroBooNE are both handled by GENIE. Resonance production occurs when a neutrino interacts with a nucleon and exchanges enough energy to the nucleon to change the isospin of one or more of its constituent quarks, elevating the nucleon to a higher energy "resonant state". Multiple resonant states of increasing energy exist for each nucleon, with the $\Delta^{+}$and $\Delta^{0}$ resonances being the lowest energy resonances $(1232 \mathrm{MeV}[19])$ for the proton and neutron respectively. In GENIE the production of resonances (such as the $\Delta$ ) for both CC and NC interactions is modeled using the Rein-Sehgal model. This model uses the Feynman-KislingerRavndal relativistic quark model, which treats resonances as excited 3-quark states in a relativistic harmonic oscillator potential with spin-flavour symmetry, to describe neutrino-induced single pion production. The Rein-Sehgal model calculates the helicity amplitudes from the Feynman-Kislinger-Ravndal model and uses them to determine the cross-sections for neutrino-induced resonance production. All known resonances in the $W<2 \mathrm{GeV}$ region are considered. The calculations performed by the GENIE implementation of the Rein-Sehgal model are free-nucleon calculations and ignore interference between neighbouring resonances.

Directly following NC resonant $\Delta$ production is the decay of the $\Delta$. In older versions of GENIE a hard cutoff was placed on the $\Delta$ decay requiring the invariant mass of the $\Delta, W_{\Delta}$, to be greater than the mass of its $\Delta \rightarrow N+\pi$ decay channel products, $W_{\Delta}>m_{N}+m_{\pi}$. This is acceptable for the $\Delta \rightarrow N+\pi$ decay channel but the $\Delta \rightarrow N+\gamma$ channel does not produce a pion and should therefore have a cutoff of $W_{\Delta}>m_{N}$. As such, any potential radiative decays in the $m_{N}$ to $m_{N}+m_{\pi}$ region were suppressed in GENIE, resulting in a minor deficit to the overall $\Delta \rightarrow N+\gamma$ decay rate at lower neutrino energies. As of GENIE v2.12.0 $\Delta$ decays are properly handled by first calculating $W_{\Delta}$ and then suppressing only the decay channels in which the sum of the decay product masses are greater than $W_{\Delta}$. Should the radiative decay channel be selected by GENIE, the $\Delta$ will be decayed isotropically into a nucleon and photon. The modeling of $\Delta$ radiative decay as isotropic by GENIE is 
an approximation, as the Rein-Sehgal model itself dictates that $\Delta$ radiative decay is anisotropic [76]. For the analysis in this thesis GENIE uses the PDG 0.6\% branching fraction for $\Delta$ radiative decay in its modified $\Delta$ decay calculations.

Following the the initial neutrino-nucleus interaction are final state interactions (FSIs). FSIs are interactions between the particles produced by the initial neutrino interaction and the composite particles of the nucleus. The analysis uses the GENIE default INTRANUKE/ $h A$ model [56], a data-driven model for simulation of FSIs in the nucleus. This model handles the final state interactions of radiatively produced nucleon and photon $\Delta$ decay products differently. The radiatively-produced nucleon will undergo GENIE simulated final state interactions such as rescattering but cannot be re-absorbed into the nucleus. The radiatively-produced photon undergoes no final state interactions in GENIE and will simply exit the nucleus in the same state it was produced. This ignores the potential for the photons momentum to be changed and also the potential for photon and nucleon reabsorption into the nucleus.

Photons emission with energies of order $\mathcal{O}(10) \mathrm{MeV}$ can also occur via the deexcitation of a nucleus or nuclear fragment. In GENIE v2.12.2 this process has only been implemented for oxygen due to the prevalence of water Cherenkov detectors in which such photons can significantly impact energy reconstruction. The expected NC $\Delta$ radiative photon energies in MicroBooNE are of $\mathcal{O}(50-100) \mathrm{MeV}$ so the missing implementation of this process is not likely to impact the LEE photon search.

Figure 4.2 shows the cross-sections used by GENIE to generate $\Delta^{+}$and $\Delta^{0}$ resonances on ${ }^{40} \mathrm{Ar}$ and ${ }^{12} \mathrm{C}$ targets via NC interactions. These two neutrino targets are the detector targets of MicroBooNE and MiniBooNE respectively allowing for comparison of the differing neutrino interaction cross-sections between the two experiments. There are two main differences in cross-section between argon and carbon. The first of which is the difference in nucleon number: argon has more than double the number of nucleons that carbon has, giving argon a larger total crosssection. The second difference is that carbon consists of an equal number of protons and neutrons making the cross-sections for neutrino-induced resonant $\Delta^{+}$and $\Delta^{0}$ production on carbon equal. Argon has a greater number of neutrons than protons, affording an incoming neutrino more opportunity to interact with a neutron and thus giving the $\Delta^{0}$ cross-section a greater value than that of the $\Delta^{+}$.

Figure 4.3 shows the relative of the estimated $\nu_{\mu}$ induced NC resonant $\Delta$ production and subsequent radiative decay cross-section to the total $\nu_{\mu}$ interaction cross-sections in terms of true neutrino energy (in GENIE). The radiative decay 


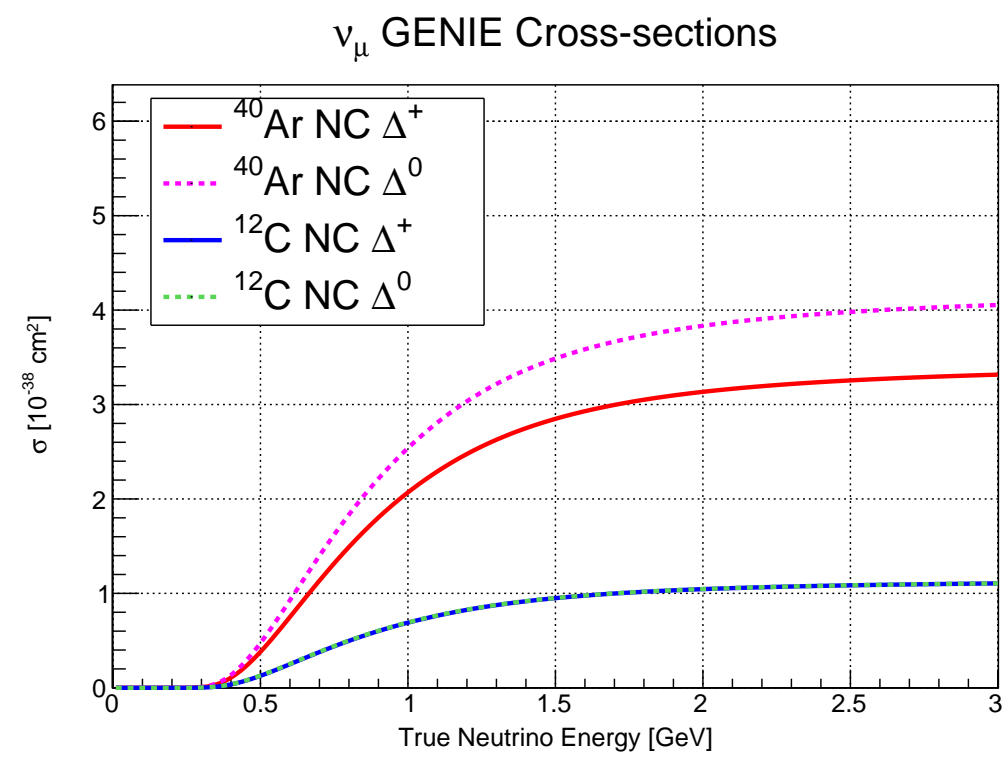

Figure 4.2: GENIE interaction cross-sections for $\nu_{\mu}$ induced NC resonant $\Delta^{+}$and $\Delta^{0}$ production on argon and carbon in terms of true neutrino energy. Considers all nucleons in the argon and carbon nuclei (not per-nucleon).

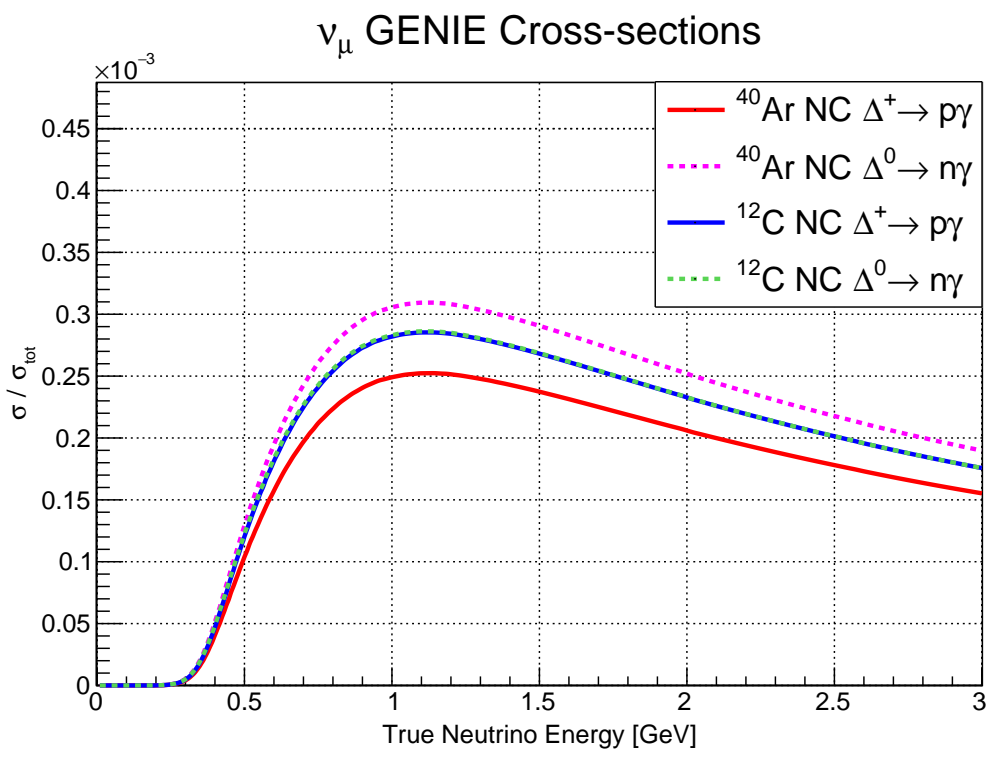

Figure 4.3: Ratio of estimated $\nu_{\mu}$ induced NC resonant $\Delta$ production and subsequent radiative decay cross-sections to the total $\nu_{\mu}$ interaction cross-sections in terms of true neutrino energy.

cross-section is estimated by scaling the $\nu_{\mu}$ induced NC resonant $\Delta^{+}$and $\Delta^{0}$ crosssections shown in Figure 4.2 by the $0.6 \%$ branching ratio prediction from the PDG (see Subsection 2.3.1). This is a simple estimation that does not take into account the $W_{\Delta}$ dependence mentioned above and assumes all $\Delta$ s are produced at the resonance pole. The figure shows that the estimated cross-section for $\Delta^{+}$and $\Delta^{0}$ ra- 
diative decay is roughly three orders magnitude smaller than the total cross-section on both carbon and argon, making neutrino induced NC $\Delta$ radiative decay a very rare process in both MiniBooNE and MicroBooNE.

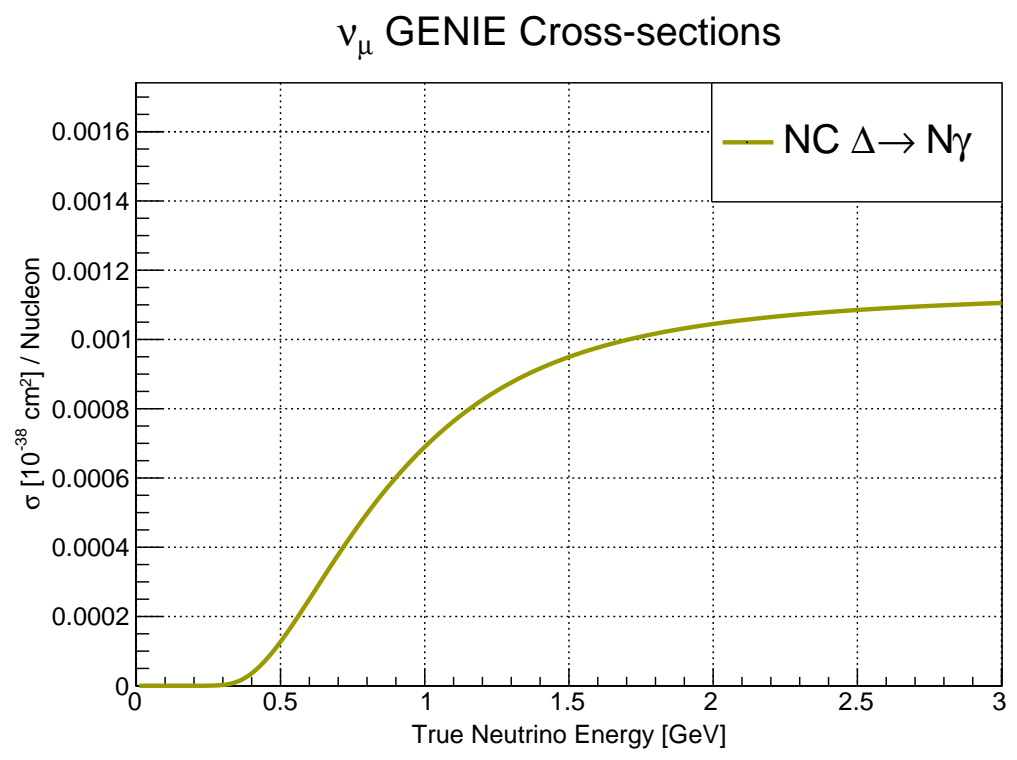

Figure 4.4: Estimated $\nu_{\mu}$ induced NC resonant $\Delta$ production and subsequent radiative decay cross-section per nucleon in terms of true neutrino energy.

Figure 4.4 shows the estimated $\nu_{\mu}$ induced NC resonant $\Delta$ production and subsequent radiative decay cross-section per nucleon. This cross-section is estimated using the same method as for Figure 4.3. An estimate for the NC $\Delta$ radiative LEE interpretation cross-section in GENIE can be determined by multiplying this cross-section by the $\times 3$ LEE enhancement factor (see Section 2.3.1). Given the true neutrino energy of the unfolded NC $\Delta$ radiative LEE in MiniBooNE peaks at approximately $1 \mathrm{GeV}$ (see Figure 2.4), a rough estimate for this cross-section in GENIE is $0.0021 \times 10^{-38} \mathrm{~cm}^{2} /$ nucleon. This is well below the neutrino-induced single photon cross-section limit of $0.0903 \times 10^{-38} \mathrm{~cm}^{2} /$ nucleon set in T2K [28].

\subsubsection{Systematic Uncertainties}

While GENIE uses the Rein-Sehgal model to simulate NC neutrino-induced $\Delta$ resonance production followed by the isotropic radiative decay of the $\Delta$ into a photon and nucleon to simulate NC $\Delta$ radiative decay, various other models for NC $\Delta$ radiative decay exist. Such models are included in the inclusive NC neutrino induced single photon production models shown in Figure 4.1. These include theoretical predictions from Hill [72], Wang et al. [74] and Zhang and Serot [75] as well as the NUANCE and NEUT MC event generators. Changes to the modeling of NC $\Delta$ res- 
onance production and subsequent radiative decay will affect predicted cross-section and final state photon and nucleon kinematics and so will factor in to the systematic uncertainty of the signal prediction.

Knowledge of FSIs in the nucleus post neutrino interaction is currently limited and thus serves as a potentially large source of uncertainty for the analysis. These FSIs will affect neutrino interaction final state particles and their kinematics. In terms of NC $\Delta$ radiative decay, GENIE only simulates FSIs on the produced nucleon but does not consider the possibility of nucleon reabsorption into the nucleus. In reality both $\Delta$ radiative photon and nucleon would be subject to FSIs and reabsorption into the nucleus, so this is an additional factor to consider for the FSI systematic uncertainty on the signal prediction. Initial estimates from the MicroBooNE analyses place the GENIE FSI systematic uncertainty at approximately 10 $\%$ [55].

Studies were performed in the Short Baseline Neutrino (SBN) proposal [53] to estimate the expected cross-section uncertainties for neutrino-argon interactions in GENIE. These studies involved the $1 \sigma$ gaussian variation of numerous GENIE model parameters over 250 "cross-section universes". The parameters varied include the axial masses for CCQE, CC resonant and NC resonant interactions and the NC normalization factor (GENIE FSI uncertainties were not considered). The SBN studies estimated the GENIE cross-section uncertainty to be approximately $20 \%$. Certain parameters in GENIE (and their uncertainties) are particularly relevant to the analysis and were not included in the SBN studies. The first of these is the branching ratio for radiative resonance decays, $x_{B R}^{R \rightarrow X+1 \gamma}$. This is the parameter that dictates the ratio of $\Delta \rightarrow N+\gamma$ to $\Delta \rightarrow N+\pi$ decays and GENIE places the relative uncertainty of $x_{B R}^{R \rightarrow X+1 \gamma}$ at $50 \%$. The second parameter is the pion charge exchange probability, $x_{c e x}^{\pi}$. A charged pion produced in the nucleus, for example from $\Delta \rightarrow n+\pi$ decay, can exchange charge with a nucleon in the nucleus resulting in the production of a $\pi^{0}$ and thus contribution to background. GENIE places the relative uncertainty of $x_{c e x}^{\pi}$ at $50 \%$.

\subsection{Pandora Reconstruction}

The Pandora Software Development Kit for Pattern Recognition [77] is used to reconstructed tracks and showers used for the analysis. Pandora performs reconstruction in main two stages. PandoraCosmic is the first stage which prioritizes the reconstruction of hits into long tracks in an attempt to remove cosmic muons. Long reconstructed tracks are then passed to a specialised cosmic identification algorithm 
which determines which tracks are likely cosmic in origin. Any hits associated with cosmic tagged tracks are removed from further consideration and the remaining hits are handed to the following stage. PandoraNu is the second stage and is focused on identifying tracks and showers from neutrino interaction vertices. PandoraNu will search for tracks and showers that appear to have a originated from a common vertex and reconstruct accordingly. The analysis in this thesis does not use the Pandora found vertex but instead uses a vertex reconstruction algorithm designed and optimized to find single photon neutrino vertex candidates (see Section 5.1).

Reconstruction in PandoraCosmic begins with the 2-d reconstruction of hits into "clusters" on each wire plane. Ionisation electrons from tracks and showers will be drifted towards MicroBooNE's wire planes producing (ideally) three sets of hits read out by the detector (one set of hits for each wire plane). Hits that are arranged in continuous lines on a given plane will be grouped together into clusters. Any significant deviations from a continuous line, such as a neutrino vertex producing two tracks in different directions, splits caused by showering particles or secondary vertices will end reconstruction of the first cluster and start reconstruction of a second. This results in a large number of high purity clusters, with the potential for multiple clusters on the same plane being produced by the same particle. Following the initial clustering stage, Pandora attempts to merge clusters on the same plane together with the aim of creating a single cluster containing all hits produced by a single particle. Cluster merging is performed by searching for pairs of clusters which are close together or point toward each other. Conversely clusters produced by the initial clustering stage can also be split in two if they are found to significantly change direction, multiple clusters intersect or a cluster points toward the middle of another cluster. Figure 4.5 shows a MC NC $\Delta$ radiative event on each of the three wire planes.

Following cluster merging, 3-d track reconstruction is performed with the goal of associating clusters on different planes that were produced by the same particle into a single 3 -d track-like object. This is done by comparing all possible combinations of the clusters for each plane and ranking the likelihood each cluster combination has of being produced by the same particle. If there are significant discrepancies between cluster combinations, multiple algorithms can be applied to make modifications to the 2-d clustering reconstruction stage that result in cluster combinations consistent with a 3 -d track object. The resulting track-object will typically be associated with clusters from all three planes, although some may only be associated with clusters on one or two of the planes. The 3-d position of each hit associated with the track is then extrapolated using a method based on the qualities of the 

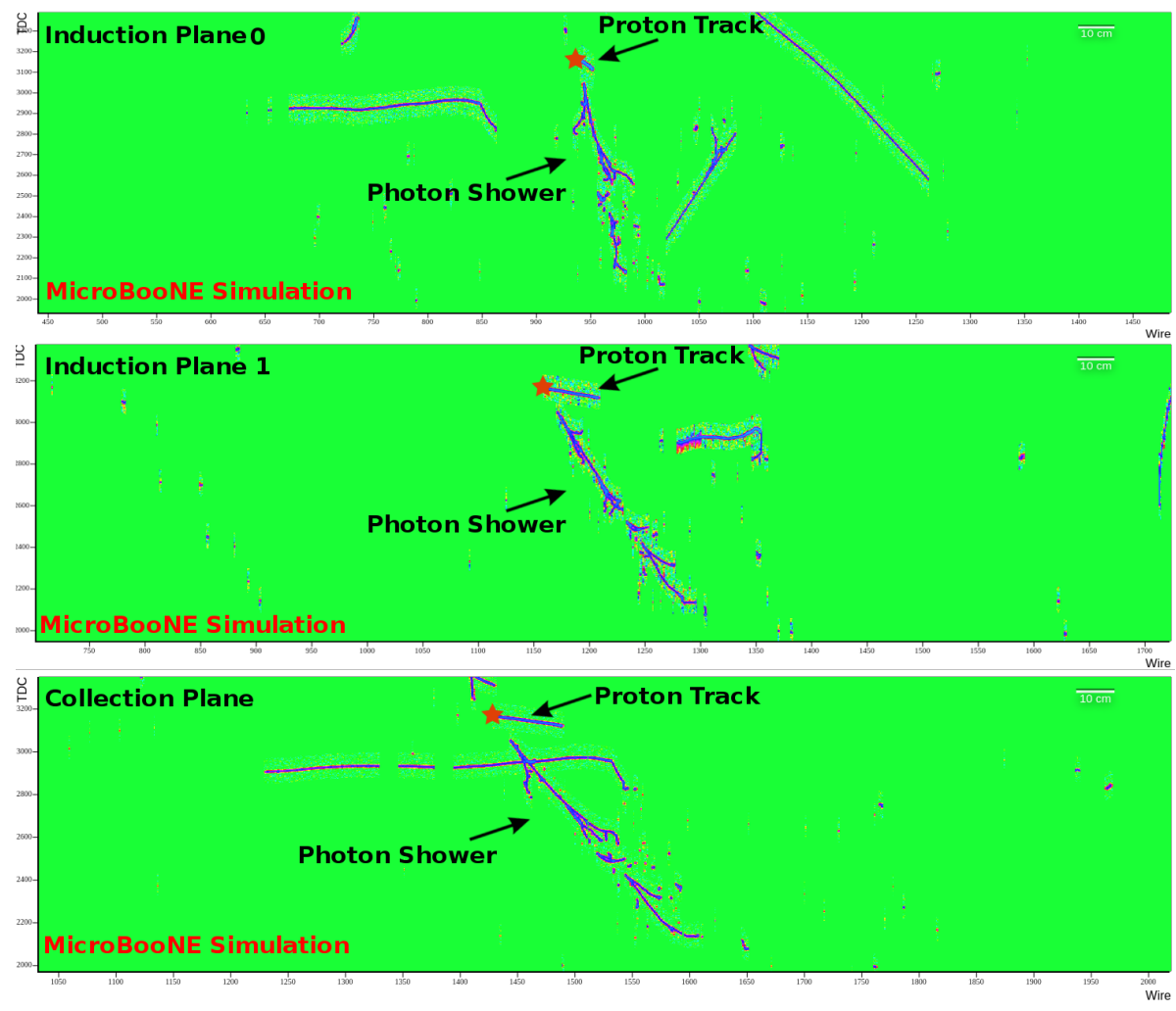

Figure 4.5: An example of a MC NC $\Delta$ radiative event with $\mathrm{MC}$ cosmics on each wire plane [78. The $x$-axis is the wire id number for each wire on the plane, the $y$-axis is the time measured in discrete time ticks (see Subsection 3.2.2).

track. These qualities include the number of planes the associated clusters of a track reside on, whether the track travels towards or parallel to the wire planes and others. Following 3-d track reconstruction, PandoraCosmic will attempt to assign vertices to the tracks which are close to the TPC ceiling (under the assumption the tracks are cosmic muons). The reconstructed tracks are handed to the subsequent cosmic identification algorithm which then removes hits associated with tracks that appear to be cosmic-like. The remaining hits are handed to the PandoraNu stage.

PandoraNu begins with the same 2-d cluster reconstruction and merging/splitting used by PandoraCosmic. The resulting clusters are then used to find candidate vertices by comparing pairs of clusters on different planes and searching for overlap on the common detector axis. If the cluster pair is found to have an overlap, the end points of the clusters are compared to determine the likely 3-d position of the candidate vertex. If this 3 -d position is not near at least one hit or in a region of unresponsive wires on all three planes then the candidate vertex is discarded. Multiple candidate vertices are produced per event and one must be selected as the probable candidate neutrino vertex. The selection of this vertex is based on the transverse energy of the associated clusters, an asymmetry score that rejects candidate vertices incorrectly located in the middle of a cluster and a beam de-weighting score that 
uses knowledge of the beam direction to preferentially select candidate vertices with lower z-coordinates. If a candidate vertex passes all these cuts and is found to be located on one or more clusters those clusters will be split into two at that location.

Once the candidate neutrino vertex has been selected, track and shower reconstruction is performed. PandoraNu uses the same reconstruction method as PandoraCosmic. Shower reconstruction is performed by first tagging clusters as either track-like or shower-like based on the length of the cluster, how transverselydistributed associated hits are compared to the direction of the cluster and the closest approach the cluster makes with the candidate neutrino vertex. If a cluster belonging to a reconstructed track are found to be shower-like, the track is discarded and the clusters are re-considered for shower reconstruction. Following the identification of shower-like clusters is the 2-d clustering of showers on each plane. This begins with the selection of long shower-like clusters that point back to the candidate neutrino as shower "spines". Shorter clusters are then added to the main shower spine cluster as shower "branches". These branches can also have shower-like clusters associated with them to create additional branches of the shower. The process of adding shower-branches to the shower spine and subsequent shower branches is performed recursively until no more clusters can be added. After shower clustering has been performed, resulting shower clusters on each plane are associated between planes into 3-d shower objects. 3-d hit reconstruction for a shower-like particles is performed for a hit on a given plane by considering all possible combinations of hits on the other two planes in close proximity, calculating a $\chi^{2}$ value for each 3 -d position and hit combination and choosing the hit combination with the best $\chi^{2}$ value.

The final step of PandoraNu reconstruction is to produce a particle hierarchy that describes the parentage of the reconstructed particles. First a "neutrino particle" is created at the selected candidate neutrino vertex. The 3 -d hits associated with all PandoraNu reconstructed tracks and showers are considered and any tracks or showers found to be associated with the candidate neutrino vertex are set as primary children of the neutrino (i.e. they are final state particles produced by the neutrino interaction). This involves performing 3-d sliding linear fits and determining both transverse and longitudinal impact parameters between the 3-d hits associated with each track and shower and the candidate neutrino vertex. Secondary parentage of any unassociated particles is then considered. For example, a CC $\nu_{\mu}$ will produce a long muon track that subsequently decays, producing a Michel electron. The muon track will be associated with the candidate neutrino vertex and therefore set as a primary child of the neutrino. The Michel electron will be set as a child of the muon 
track.

In terms of particle association, photon showers are a special case due to the expected $14 \mathrm{~cm}$ conversion length between shower start and the vertex that produced the photon. The resulting gap between shower start and vertex greatly increases the difficulty of correctly associating the photon shower to the vertex that produced it. Reconstructed showers in PandoraNu are "pointed backwards" to determine if a shower is associated with a candidate neutrino vertex or another reconstructed particle. If such an association is found then the PandoraNu particle hierarchy will be set accordingly. If no association is found then PandoraNu will by default associate the shower with the candidate neutrino vertex and set the shower as a primary child of the neutrino particle. This ensures photon showers that are truly associated with the neutrino vertex (for example from neutrino-induced $\pi^{0} \rightarrow 2 \gamma$ decay) are never missed due to the difficulty in correctly associating them. This approach can, however, also result in the incorrect association of reconstructed showers that are not truly associated with the neutrino vertex (such as cosmic showers that pass the PandoraCosmic rejection stage).

\subsection{Shower Kinematic Variables}

The reconstructed shower objects used in the analysis are produced by PandoraNu and are characterised by a 3-d starting position, direction (unit vector), length and opening angle. Length and opening angle are variables output by PandoraNu that describe the shower in terms of a 3 -d cone with direction equal to the direction of the shower. The clusters and corresponding sets of hits on each plane the shower consists of are also associated with the reconstructed shower object.

\subsubsection{Shower Energy}

The energy of a reconstructed shower is determined based on the method outlined in Ref [46], using the calorimetric information of the shower. This calculation uses the calibration constants described in Table 3.2 of Subsection 3.2.2. A separate energy value is calculated for each wire plane the shower reside on. For each plane, the integrated ADC counts of each hit associated with the shower is summed and multiplied by various calibration factors to reconstruct the energy of the shower according to

$$
E_{\mathrm{Reco}}=\frac{w_{\mathrm{Ar}}}{R} \frac{n_{\mathrm{ADC}}}{C_{p}}
$$

where: 
- $E_{\text {Reco }}$ : Reconstructed shower energy on a given plane $(\mathrm{eV})$.

- $w_{\text {Ar }}: 23.6 \mathrm{eV}$ of work required to ionise a single argon atom.

- $R$ : Recombination factor, $R=0.62$ (see Subsection 3.2.2).

- $n_{\mathrm{ADC}}$ : Integrated ADC counts of all hits associated with the shower on a given plane.

- $C_{p}$ : Calibration factor converting from ADC counts to number of ionisation electrons (see Table 3.2 of Subsection 3.2.2). Varies depending on plane and use of data/MC.

This is a rearrangement and combination of Equations 3.1 and 3.2 in Subsection 3.2 .2 .

\subsubsection{Shower $\mathrm{dE} / \mathrm{dx}$}

The shower $\mathrm{dE} / \mathrm{dx}$ calculation method used for this analysis is based on the method used by ArgoNeuT [79]. As with shower energy, the $\mathrm{dE} / \mathrm{dx}$ of a reconstructed shower is calculated for each wire plane the shower resides on. In order to calculate the $\mathrm{dE} / \mathrm{dx}$ of the start of the shower, a $4 \times 2 \mathrm{~cm}^{2}$ bounding box is created starting at the beginning of the associated cluster and pointing in the direction of the cluster. The purpose of this bounding box is to enclose all hits associated to the cluster that are part of the trunk of the shower. This is where the shower will differ in production via either pair production in the case of a photon or immediate ionisation in the case of an electron (as described in Subsection 3.2.3). The energy of each hit, $E_{\text {hit }}$, in the bounding box is calculated using the same method as in Section 4.3.1. The distance between hits in the trunk of the shower is the "effective" spacing of the wires (wire pitch) on the plane relative to the direction of the cluster. The wire pitch is calculated using

$$
d_{e}=\frac{d_{a}}{\left|\hat{c} \cdot \hat{w}_{p}\right|},
$$

where $d_{e}$ is the wire pitch, $d_{a}$ is the actual wire spacing $(3 \mathrm{~mm}), \hat{c}$ is the direction of the cluster, $\hat{w}_{p}$ is the direction perpendicular to the wires on the plane the cluster is located. The $\mathrm{dE} / \mathrm{dx}$ value for the hit is then calculated using

$$
\frac{d E}{d x}=\frac{E_{h i t}}{d_{e}} .
$$

The median $\mathrm{dE} / \mathrm{dx}$ of the hits contained in the bounding box is selected as the $\mathrm{dE} / \mathrm{dx}$ of the shower. 


\subsection{Optical Flash Reconstruction}

PMT information can be used to reconstruct "flashes" of light in the TPC that correspond to neutrino interactions and charged particles traversing the detector. PMT hits that are time-coincident within a $100 \mathrm{~ns}$ are grouped together into a "reconstructed flash". The $y$ and $z$ positions of the flash in the TPC are calculated for each axis by mapping each PMT to its location on the axis and calculating the average of PMT axis position weighted by the PE observed by each PMT. Each flash is characterised by the total PE of all associated PMT hits, the time of the flash in $\mu$ s relative to the BNB hardware trigger arrival and the $y$ and $z$ positions of the flash. 


\section{$5 \quad$ Neutrino Induced NC $\Delta$ Radiative Event Selection}

This chapter describes the multi-stage event selection process used to select NC $\Delta$ radiative events while minimizing selected background events from BNB and cosmic sources. There are three stages to the event selection process. The first is vertex reconstruction using the reconstructed tracks and showers produced by PandoraNu as input. The second is a series of pre-selection cuts primarily to reduce the significant cosmic background and to ensure good quality reconstructed tracks and showers. The final stage uses boosted decision tree (BDT) background rejection trained on both cosmic-only and BNB background events to maximize selected signal versus background. The selection described in this chapter was developed as part of a MicroBooNE public note 78].

\subsection{Vertex Reconstruction}

\subsubsection{Overview}

The vertex reconstruction algorithm uses pre-reconstructed tracks and showers (reco-objects) produced by PandoraNu. Vertex reconstruction is divided into two main sections: track association and shower association. Track association is performed first and involves comparing the start and end point of every track with every other track start/end point. Tracks with start/end points that are within a certain distance threshold are associated together.

Once all tracks have been associated in this way, shower association is performed. The expected $14 \mathrm{~cm}$ photon conversion length (see Subsection 3.2.3) means photon showers from BNB neutrino interactions (i.e. from NC $\Delta$ radiative or $\pi^{0}$ decay) will not be attached to the true neutrino vertex. In order to associate these photon showers with their true vertex showers are backwards-projected. This backwardsprojection is a line starting at the shower vertex and pointing in the opposite to the direction of the shower. The impact parameter this line makes with other recoobjects determines if the shower is associated with those objects. Due to the heavy dependence of this backwards-projection on the uncertainty of reconstructed shower direction, track association is performed first as tracks have a well defined start and end point in the TPC and are guaranteed to be attached to the vertex that produced 
them. Once vertex reconstruction is complete, any reconstructed vertices produced with at least one associated shower are passed on to the next stage of the analysis.

\subsubsection{Track Association}

Track association begins by calculating the distances between every track start/end point and every other track start/end point (excluding start/end points from the same track) in the event. If the shortest of these distances, $d t$, is greater than a pre-defined threshold, $t_{\max }$, then no tracks are associated. Otherwise the two tracks with the closest start/end points are associated together and a candidate vertex is created at the midway point between the two track start/end points (see Figure 5.1).

If there are any other tracks with a start/end point within $t_{\max }$ of this newly created candidate vertex they are also associated with the vertex. All track start/end points associated with the candidate vertex are removed from further consideration by this stage of the algorithm. Note that this does not mean the whole track is removed, only the start/end of the track that has been associated with a candidate vertex. If the track is extremely short and its other start/end point is also within $t_{\max }$ of the candidate vertex then the this point is also removed and thus the whole track is removed from further consideration. This process is then repeated starting with the calculation of distances between every remaining track start/end point, creating another candidate vertex between the two closest remaining track start/end points and adding any other track start/end points within $t_{\max }$ of the created candidate vertex. Track candidate vertices will continue to be produced until there are no two track start/end points remaining that are within $t_{\max }$ of each other. See Figure 5.2 for a flow chart of this process.

Each start/end point of a track can be included in a reconstructed vertex and so each track can potentially be included in two different reconstructed vertices. The "output" of this section of the algorithm is a group of reconstructed vertices containing only track-track associations. Each reconstructed vertex includes the following information:

- Position of the vertex.

- Unique identifier (ID) number for each associated reco-object (only tracks at this stage).

- Reco-object start/end point associated with the reconstructed vertex.

- ID of every other reconstructed vertex it is connected to and the track by which it is connected. 


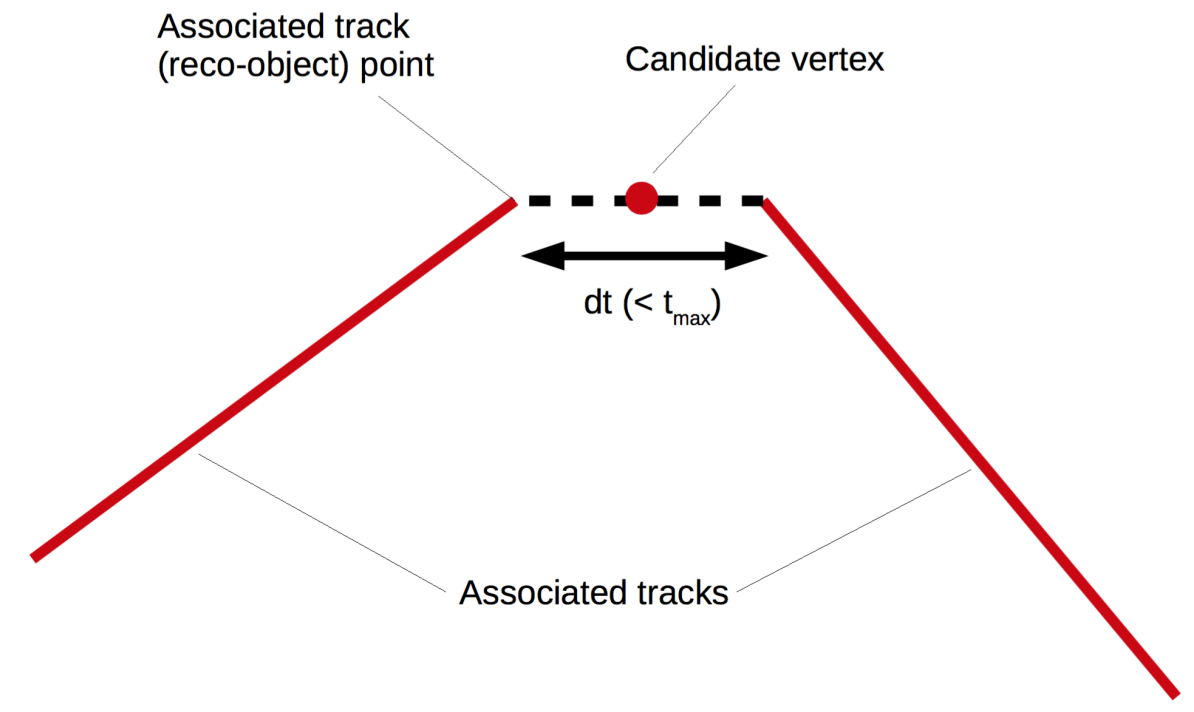

Figure 5.1: Association of two tracks and creation of reconstructed vertex at the midway point.

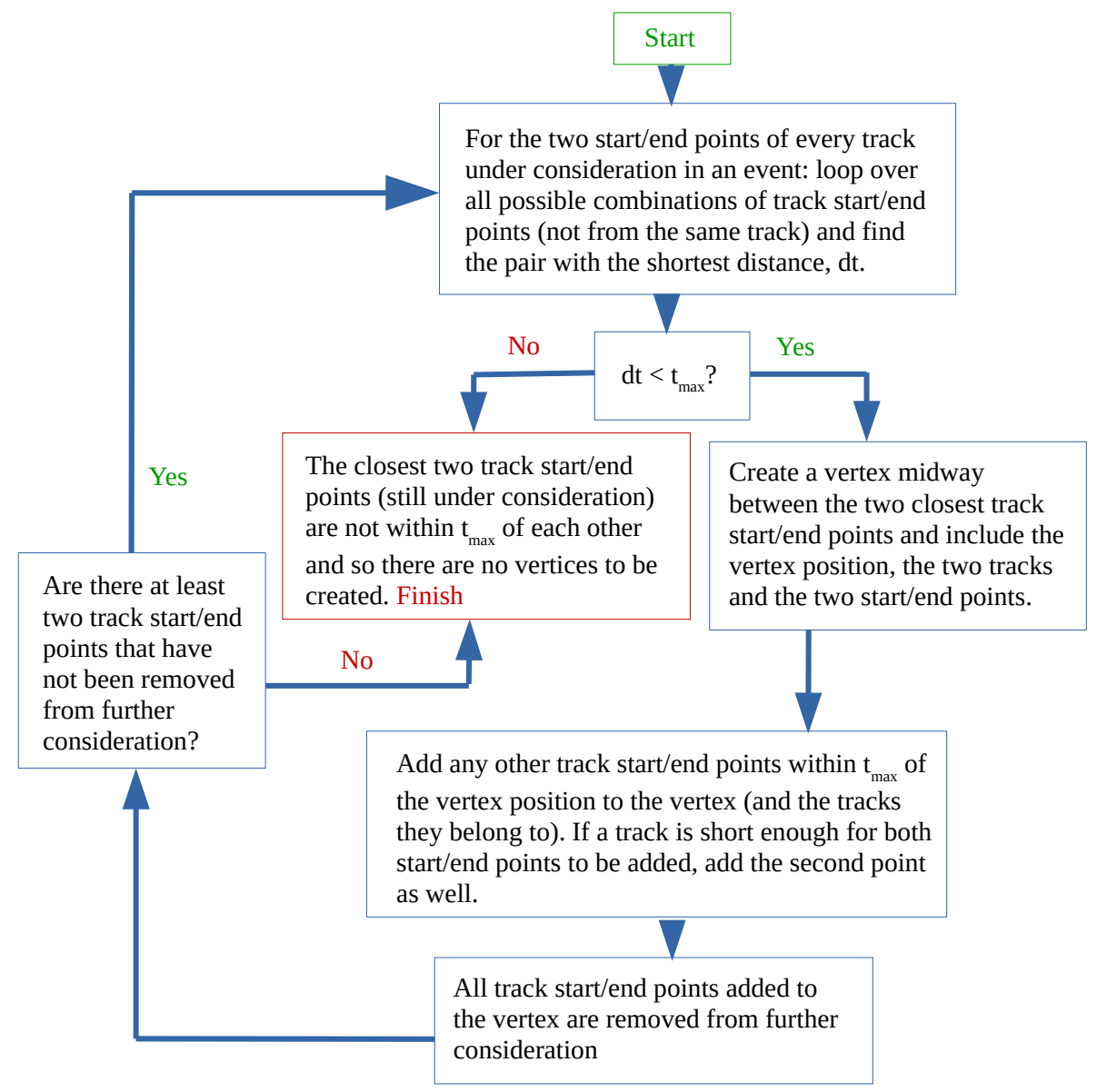

Figure 5.2: A flow chart of the track association process. 


\subsubsection{Shower Association}

Shower association begins by backwards-projecting each shower in the event. A shower can be associated with one of three objects:

- A track.

- Another backwards-projected shower.

- A pre-existing candidate vertex.

For each backwards-projection the impact parameter, $d s$, made with each other object (of the three types listed above) is calculated (see Figure 5.3 for an example involving a track). In order for the shower to be associated with another object, the distance between the shower start and the point of closest approach on the shower, $d b p$, must be less than a maximum backwards projection threshold, $b p_{\max }$. Furthermore $d s$ itself must be shorter than a maximum impact parameter threshold, $s_{\max }$. If the shower-object pair with the smallest $d s$ fulfills both these criteria then the pair are associated together and the shower is removed from further consideration. This process is repeated until there are no more shower-object pairs where $d b p<b p_{\max }$ and $d s<s_{\max }$. The manner in which a shower is associated with an object depends on what shower - object match is found. See Figure 5.4 for a flow chart of this process. 


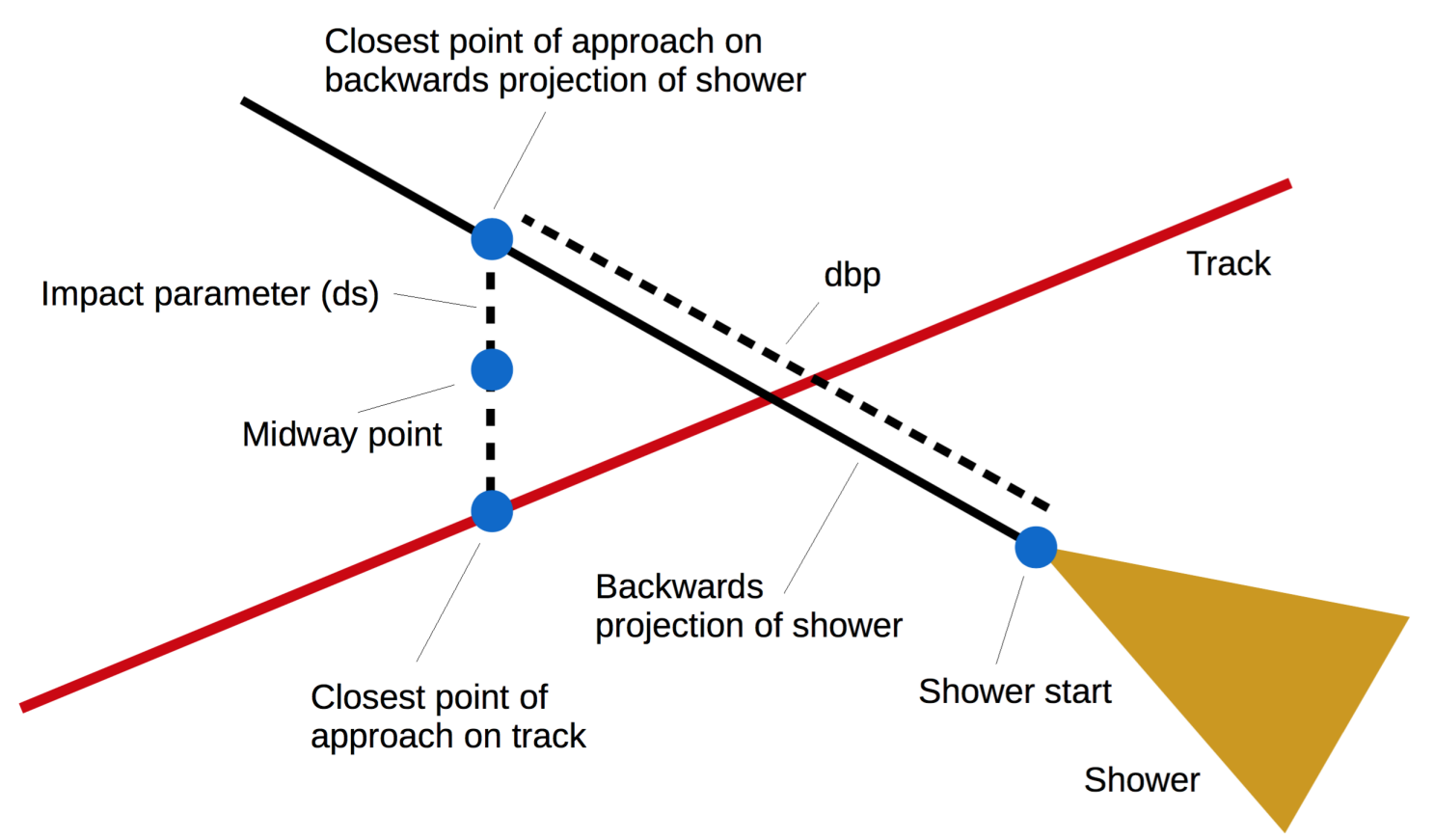

Figure 5.3: Backwards projection of a shower. A line starting at the shower start point is pointed in the opposite direction to the shower. This is the backwardsprojection of the shower. The vertical dashed line shows the impact parameter (shortest possible distance) between this line and a track. The closest points of approach on the track and backwards projection of the shower are the points at which the impact parameter intersects the track and backwards-projection of the shower respectively. The dashed line along the backward-projection is the distance between the start-point of the shower and the closest point of approach on the backward-projection, $d b p$. 


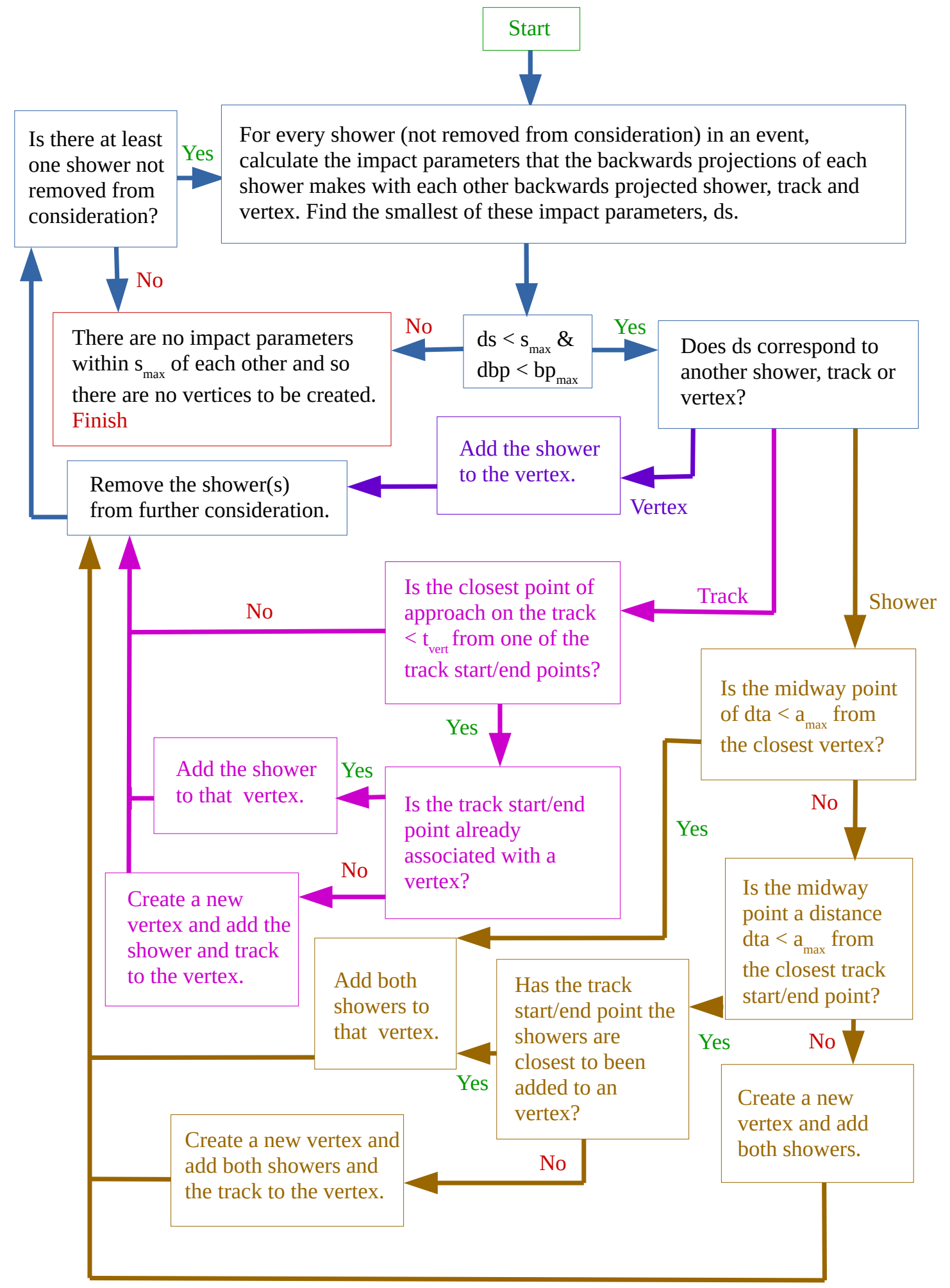

Figure 5.4: A flow chart of the shower association process. 


\section{Shower-track Matches}

In the case of a shower-track match there are four possible scenarios to consider. These are described in the pink pathway of Figure 5.4. Figure 5.5 shows the first scenario, where a shower is associated with the start/end of a lone track. Such a scenario will arise from the following processes:

- NC $\boldsymbol{\Delta}^{+} \rightarrow \boldsymbol{p}+\boldsymbol{\gamma}$ : Produces a photon shower and a proton track. This is one of the two signal topologies of interest to the analysis. The other is NC $\Delta^{0} \rightarrow n+\gamma$, however this topology produces a lone photon shower and therefore will not be associated with other reco-objects.

- $\boldsymbol{\nu}_{\boldsymbol{e}}+\boldsymbol{n} \rightarrow \boldsymbol{e}^{-}+\boldsymbol{p}$ : Produces an electron shower and a proton track.

- $\boldsymbol{\mu}^{-} \rightarrow \boldsymbol{\nu}_{\boldsymbol{\mu}}+\boldsymbol{\nu}_{\boldsymbol{e}}+\boldsymbol{e}^{-}$: Many muons produced by $\nu_{\mu}+n \rightarrow \mu^{-}+p$ and cosmic muons will decay into Michel electrons before they exit the TPC, leaving an electron shower at the end of a muon track.

- $\boldsymbol{\nu}_{\boldsymbol{\mu}}+\mathrm{Ar} \rightarrow \boldsymbol{l}+\boldsymbol{\pi}^{\mathbf{0}}+\boldsymbol{X}$ : Both $\mathrm{CC}$ and $\mathrm{NC}$ neutral pion production can produce a single track and two photon showers. For the CC interaction $l=\mu^{-}$, producing a muon track. For the NC interaction $l=\nu_{\mu}$ but FSIs can result in the production of one (or more) additional tracks with which the shower can be associated. This scenario describes the association of the first of the two photon showers (the shower with the smallest shower-track $d s$ ) to be associated with the track. The second photon shower (the shower with the larger showertrack $d s$ ) will be associated in a subsequent iteration of the shower association stage. Physics and reconstruction based effects mean it is possible for only one of the photon showers to be reconstructed in the TPC, in which case the track and only a single photon shower will be observed.

For this scenario, as shown in Figure 5.5, the closest point of approach the shower makes on the track is within a distance threshold $t_{\text {vert }}$ from one of the start/end points of the track. This indicates the track and shower share a common vertex and so a new vertex is created at the start/end point of the track and both the track and shower are added to it.

Figure 5.6 shows the second scenario, where a shower is associated with the free start/end of a track that belongs to pre-existing vertex. Such a scenario will arise from the following processes:

- $\boldsymbol{\nu}_{\boldsymbol{\mu}}+\boldsymbol{n} \rightarrow \boldsymbol{\mu}^{-}+\boldsymbol{p}$ : Produces a muon and proton track. The muon track subsequently decays, producing a Michel electron shower. 


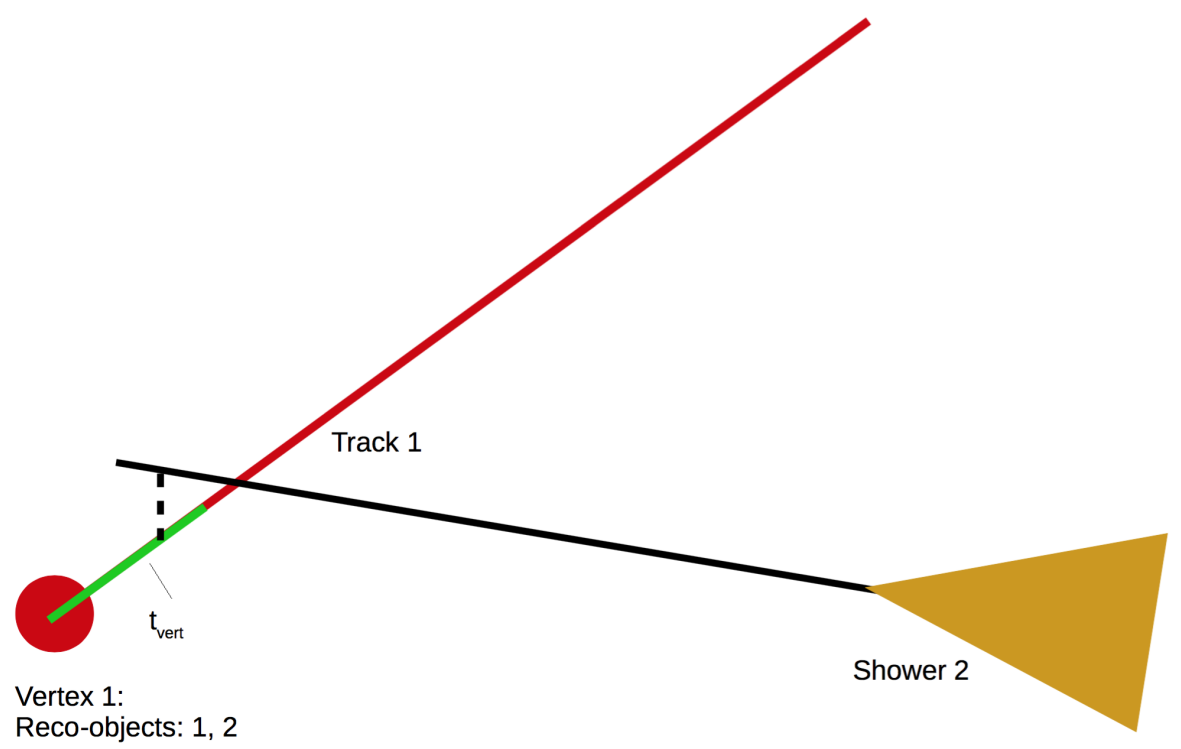

Figure 5.5: Association of a shower and a lone track. The backwards-projection of shower 2 in this instance is found to have the smallest impact parameter with track 1. As point of closest approach on the track is within $t_{v e r t}$ of one of the start/end points of track 1 , the shower is assumed to share a common vertex with the track. Vertex 1 is created at the start/end point of the track and both track 1 and shower 2 are added to it.

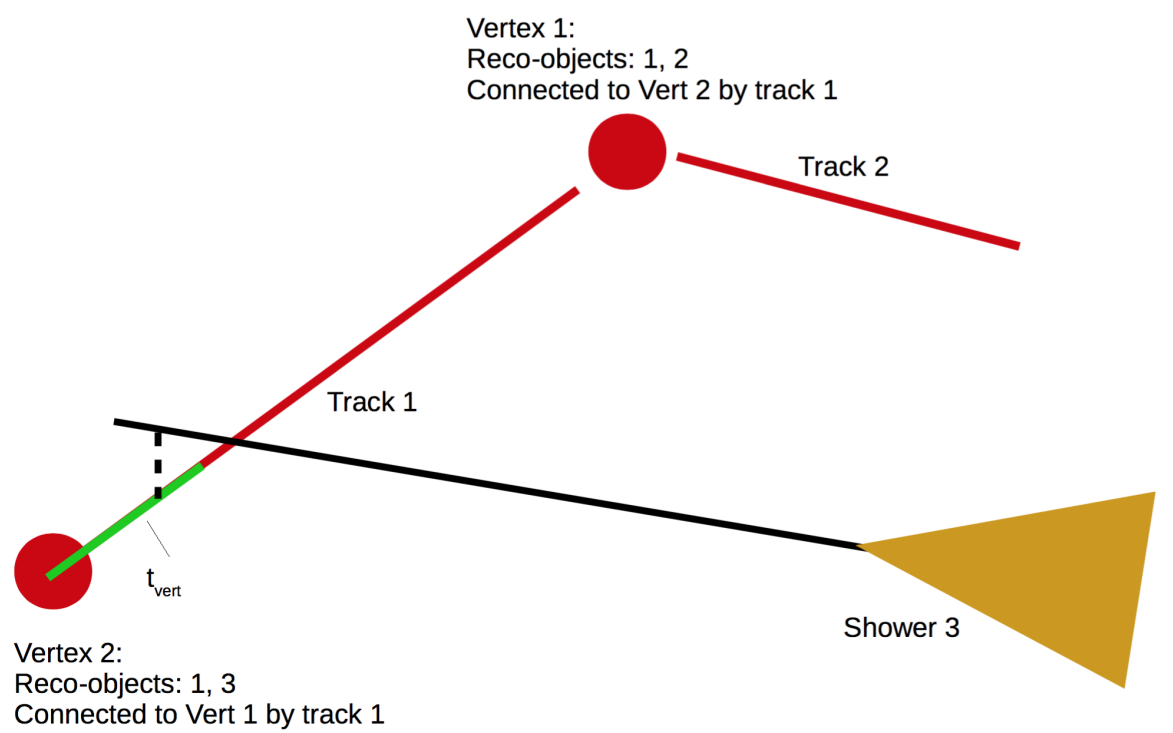

Figure 5.6: Association of a shower and a track already associated with a pre-existing vertex. The backwards-projection of shower 2 in this instance is found to have the smallest impact parameter with track 1 . As the point of closest approach on the track is within $t_{\text {vert }}$ of one of the start/end points of track 1, the shower is assumed to share a common vertex with the track. Vertex 1 is created at the start/end point of the track and both track 1 and shower 2 are added to it. In addition the intervertex connection between vertices 1 and 2 and the track by which the two vertices are connected is recorded by both vertices. 
- $\boldsymbol{\nu}_{\boldsymbol{\mu}}+\mathrm{Ar} \rightarrow \boldsymbol{\mu}^{-}+\boldsymbol{\pi}^{+}+\boldsymbol{X}$ : Produces a muon and charged pion track. The muon track subsequently decays, producing a Michel electron shower.

This scenario is handled similarly to the first, where the associated track and shower (track 1 and shower 3 in Figure 5.6) are assumed to share a common vertex and so a new vertex (vertex 2 in Figure 5.6) is created and both reco-objects are added. In addition to this, the inter-vertex connection between the two vertices (vertices 1 and 2 in Figure 5.6) and the track by which the two vertices are connected (track 1 in Figure 5.6 is recorded by both vertices.

Figure 5.7 shows the third scenario, where a shower is associated with the start/end of a track that belongs to pre-existing vertex. Any of the one track one shower processes described in the first scenario can potentially also cause this scenario if an additional track is produced by FSIs. In this scenario a shower (shower 3 in Figure 5.7) is associated with a track (track 1 in Figure 5.7) that already belongs to another reconstructed vertex (vertex 1 in Figure 5.7). The closest point of approach on the track is within a distance threshold $t_{v e r t}$ from the start/end of the track that is associated with the pre-existing vertex. For this reason it is assumed that the track and shower have a common vertex and so the shower is added to the pre-existing vertex.

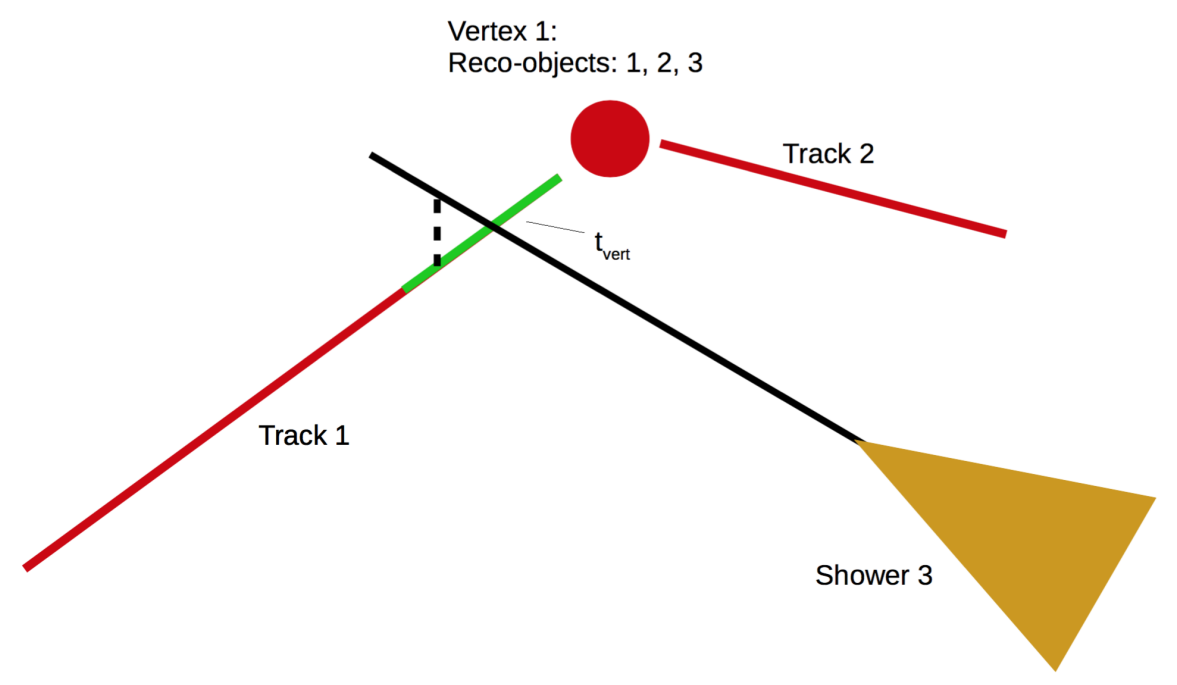

Figure 5.7: Association of a shower and a pre-existing vertex. The backwardsprojection of shower 3 is found to have the smallest impact parameter with track 1 . The point of closest approach on the track is within $t_{v e r t}$ of the end of track 1 that belongs to vertex 1 . This indicates that shower 3 also belongs to vertex 1 and so is added to it.

Figure 5.8 shows the fourth scenario, where the shower is associated with a track but not with either end of it. This only occurs when a delta ray electron shower 
is produced by a muon track. In this scenario the closest point of approach on the track is $<t_{v e r t}$ from either start/end of the track, indicating that the shower does not share a common vertex with the track but was still produced by it. As such the shower is removed from further consideration by the vertex algorithm and the analysis as a whole.

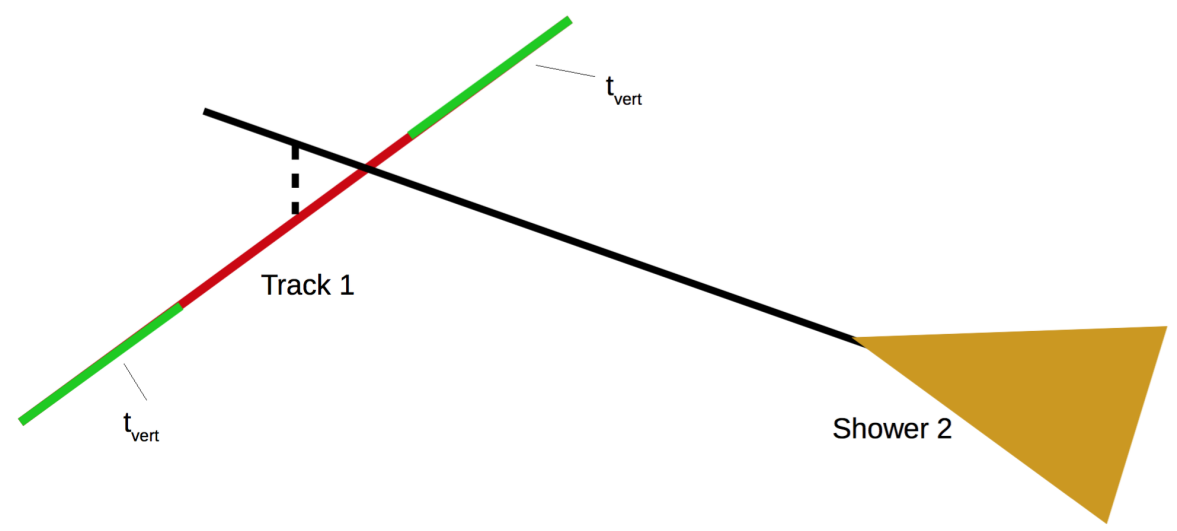

Figure 5.8: Shower appears to be a delta ray electron produced by a track. The smallest impact parameter the backwards projection of shower 3 makes is with track 1. The closest point of approach on the track is not within $t_{\text {vert }}$ of either end of track 1 , hence shower 3 is assumed to be a delta ray electron and is removed from the analysis.

\section{Shower-shower Matches}

In the case of a shower-shower match there are three possible scenarios to be considered. These are described in the gold pathway of Figure 5.4. Figure 5.9 shows the first scenario, where two showers have been associated with each other. This scenario is arises from neutrino induced NC $\pi^{0}$ production and subsequent $\pi^{0} \rightarrow 2 \gamma$ decay which produces two photon showers with a common vertex. In this scenario two showers have been backwards-projected and found to match with each other. No track is present to give an anchor to the position of the vertex, thus the position of the vertex is chosen to be the midway point of the impact parameter between the two shower backwards-projections.

Figure 5.10 shows the second scenario, where two showers have been associated with each other and their common vertex is close to the start/end of a lone track. This scenario shares similarities with the first shower-track match scenario, where a single shower is associated with a lone track. However, as this scenario is specifically for two showers, it can only be caused by $\mathrm{CC}$ and $\mathrm{NC}$ neutral pion production. For the CC interaction two photon showers and a muon track are produced, for the NC interaction two photon showers are produced and FSIs produce the track. In 


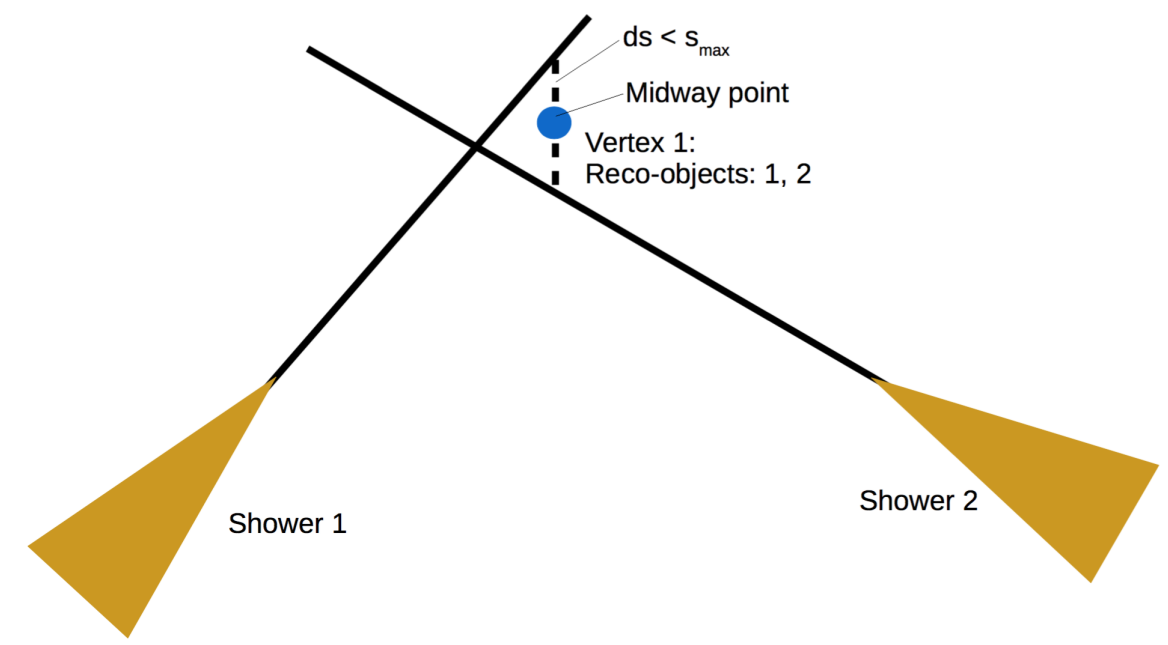

Figure 5.9: Association of two showers. Two showers have been backwards-projected and found to match with each other. A new vertex is created and both showers are added to it. The position of the vertex is chosen to be the midway point of the impact parameter (dashed line) between the two shower backwards-projections.

this scenario two showers have been backwards-projected and found to match with each other similarly to the first shower-shower match scenario. The position of the vertex is chosen to be the midway point of the impact parameter between the two shower backwards-projections, however the free start/end of a lone track is within a distance threshold $a_{\max }$ from this midway point. This indicates that the track and the two showers share a common vertex thus a new vertex is created at the free start/end of the lone track and the three reco-objects are added to it.

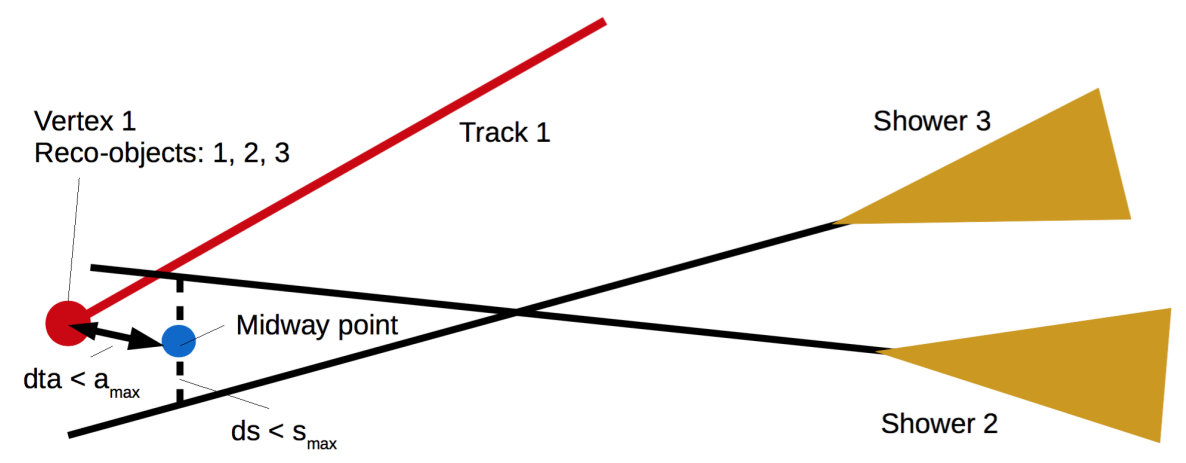

Figure 5.10: Association of two showers with a track. Showers 3 and 4 have been backwards-projected and found to match with each other. The distance between the common vertex of showers 3 and 4 and one of the start/end points of track 1 , $d t a$, is less than $a_{\max }$. A new vertex is created at the location of the start/end of track 1 and the three reco-objects are added to the new vertex.

Figure 5.11 shows the third scenario, where two showers have been associated with each other and their common vertex is close to a pre-existing vertex. As for the 
second shower-shower match scenario, this scenario can only be caused by CC and NC neutral pion. For the CC interaction FSIs must produce at least one track (in addition to the muon track) for a pre-existing vertex to be produced by the track association stage. For the NC interaction FSIs must produce at least two tracks. In this scenario two showers have been backwards-projected and found to match with each other. The midway point of this match is found to be within $a_{\max }$ of a pre-existing candidate vertex. The two showers are assumed to have originated from this vertex and are therefore added to it.

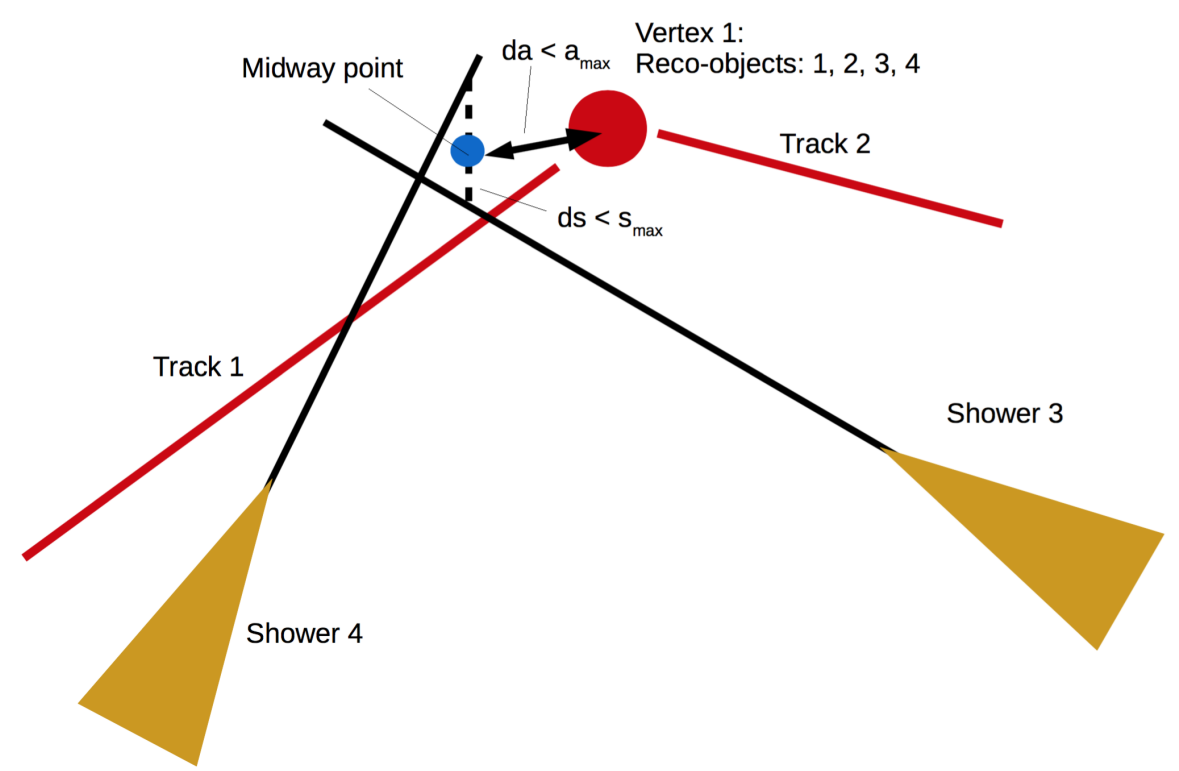

Figure 5.11: Association of two showers with a pre-existing vertex. Showers 3 and 4 have been backwards-projected and found to match with each other. The distance between the common vertex of showers 3 and 4 and and vertex 1, $d a$, is less than $a_{\max }$ and so showers 3 and 4 are added to vertex 1 .

In the case where a shower is matched most closely to a pre-existing vertex the shower is simply added to that vertex. Once track and shower association stages have been performed, any vertex with at least one associated shower is passed on to the next stage of the analysis. There can be multiple vertices per event that are output at this stage.

\subsubsection{Reconstruction - Truth Matching}

In order to determine the performance of the vertex reconstruction algorithm, the true identity of the reconstructed tracks and showers in an event is required. For this analysis the matching between true MC particles and reconstructed tracks and showers is performed by considering the true charge deposited by each true particle 
into each reco-object. It is possible for a reco-object to have charge contributions from multiple true particles. In this cases such as these, the true particle that contributes the largest amount of charge to the reco-object is the true particle that is matched to the reco-object.

\subsubsection{Optimization}

The vertex reconstruction algorithm was optimized on two different MC samples:

- BNB + cosmics: GENIE simulated BNB inclusive background MC with CORSIKA cosmic overlay MC.

- NC $\Delta$ radiative + cosmics: GENIE simulated BNB signal MC with CORSIKA cosmic overlay MC.

Each sample consisted of 10,000 events. The goal of the optimization was to find the "best performing" values for the algorithm input parameters. As a brief reminder:

- $\boldsymbol{t}_{\boldsymbol{m a x}}$ : the maximum distance at which two track start/end points can be that to be associated with one another.

- $\boldsymbol{s}_{\max }$ : the maximum impact parameter a shower backwards-projection can make with another shower, track or vertex to be associated with that object.

- $\boldsymbol{b} \boldsymbol{p}_{\boldsymbol{m a x}}$ : the maximum allowed distance between the shower start and the closest point of approach on the shower (i.e. how far back a shower can be backwards-projected).

- $\boldsymbol{a}_{\max }$ : the maximum distance the midway point of the impact parameter for two backwards-projected showers can be to be associated with a track/pre-existing vertex.

- $\boldsymbol{t}_{\boldsymbol{v} \text { ert }}$ : the maximum distance the closest point of approach a backwardsprojected shower makes on a track can be from the track start/end point to associate the shower with the track start/end point.

In order to optimize the vertex algorithm, its performance was quantified using the following three quantities:

- NReco: the number of reco-objects associated with the reconstructed vertex.

- NTrue: the number of reco-objects associated with the true neutrino vertex (reco-objects matched with true MC particles from the true neutrino vertex).

- NCorrect: the number of reco-objects associated with both the reconstructed vertex and the true neutrino vertex. 
These quantities were used to define the following performance quantities:

- Completeness: NCorrect/NTrue

- Cleanliness: NCorrect/NReco

Completeness is a measure of how many reco-objects associated with the true neutrino vertex were successfully associated with the reconstructed vertex (see Figure 5.12). In terms of the low energy single photon analysis, maximizing completeness maximizes the number of $\mathrm{NC} \Delta^{+} \rightarrow p+\gamma$ photon showers that are correctly associated to the corresponding proton track. This can be a powerful asset for signal selection as it allows the analysis to take advantage of shower-vertex gap information to identify a $\mathrm{NC} \Delta$ radiative shower as a photon. Maximizing completeness also maximizes the amount of $\pi^{0} \rightarrow 2 \gamma$ background that can be rejected by associating two photon showers to the same vertex. To a lesser extent $\nu_{e}$ CCQE and Michel electron shower background will also be reduced by maximizing completeness using shower-vertex gap information, however as such showers will almost always be attached to the vertex, the impact on these backgrounds will not be as significant.

Cleanliness is a measure of how many reco-objects associated with the reconstructed vertex truly originated from the true neutrino interaction (see Figure 5.13). In terms of the analysis, maximizing cleanliness will minimize the number of NC $\Delta$ radiative photon showers incorrectly associated with a cosmic shower and consequently rejected as two shower $\pi^{0}$ background. Similarly it will also minimize the number of NC $\Delta$ radiative photon showers incorrectly associated with a long cosmic muon track and rejected as background later in the analysis (as $\Delta$ radiative produced are proton tracks which are expected to be short).

In order to minimize signal loss and maximize background rejection at this stage of the analysis, the simultaneous maximization of completeness and cleanliness was prioritized. As such the goal of the vertex algorithm optimization was to maximize the number of reco-vertices whose reco-objects were all associated with the true neutrino event vertex (completeness=1) and were the only reco-objects associated with the true neutrino event vertex (cleanliness=1). To this end, completeness and cleanliness were combined into a single "combined performance quantity", $c p q=$ completeness $\times$ cleanliness, and the number of reco-vertices with $c p q=1$ (cleanliness $=1$ and completeness $=1)$ in the $\mathrm{NC} \Delta$ radiative + cosmics and $\mathrm{BNB}+$ cosmics samples were maximized. In order to determine what permutation of parameter values would maximize the number of reco-vertices with $c p q=1$, a multiparameter scan was performed for the four shower-related parameters simultaneously over the parameter phase-space listed below: 


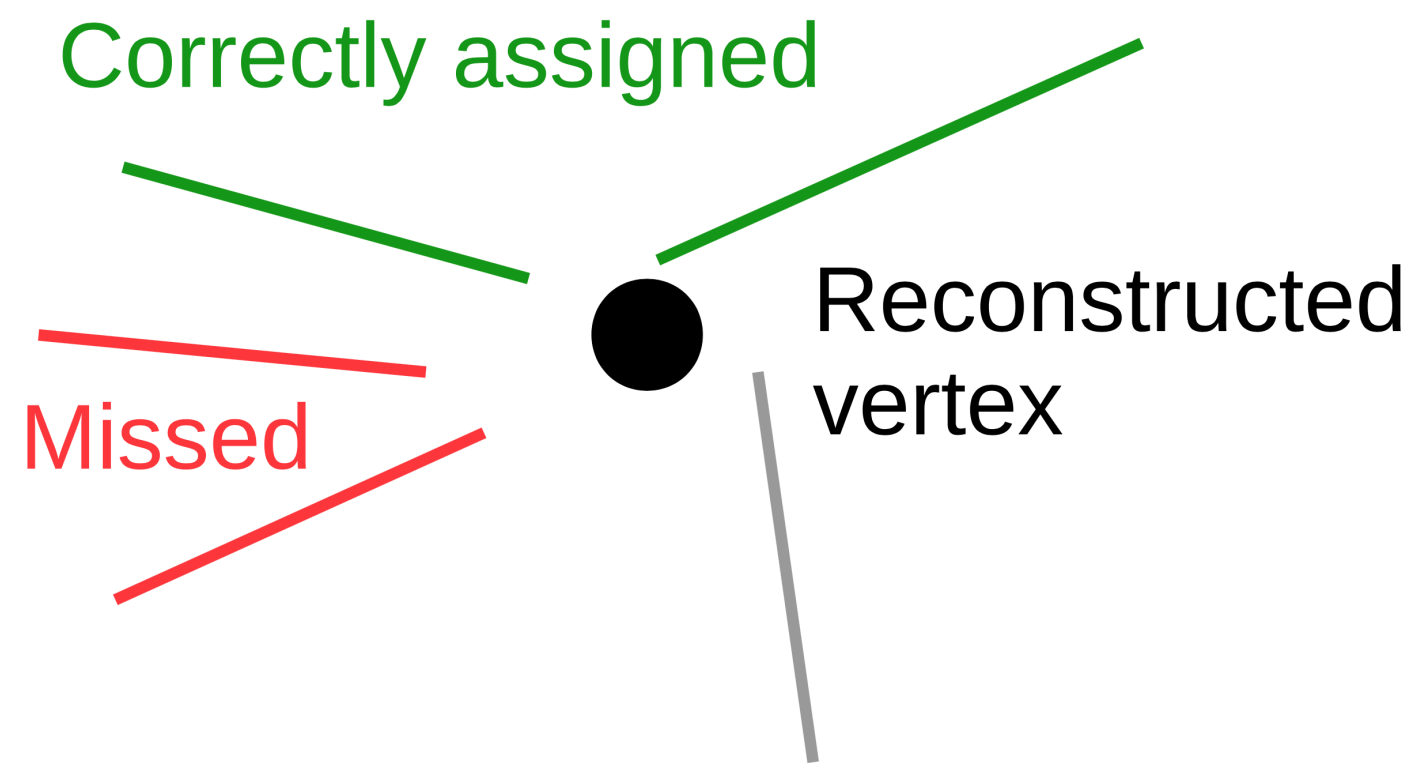

Figure 5.12: An example of reconstructed vertex completeness. In this example the true neutrino vertex has four tracks associated with it, however only two of them were correctly assigned to the reconstructed vertex. In this case the reconstructed vertex has a completeness of $2 / 4$.

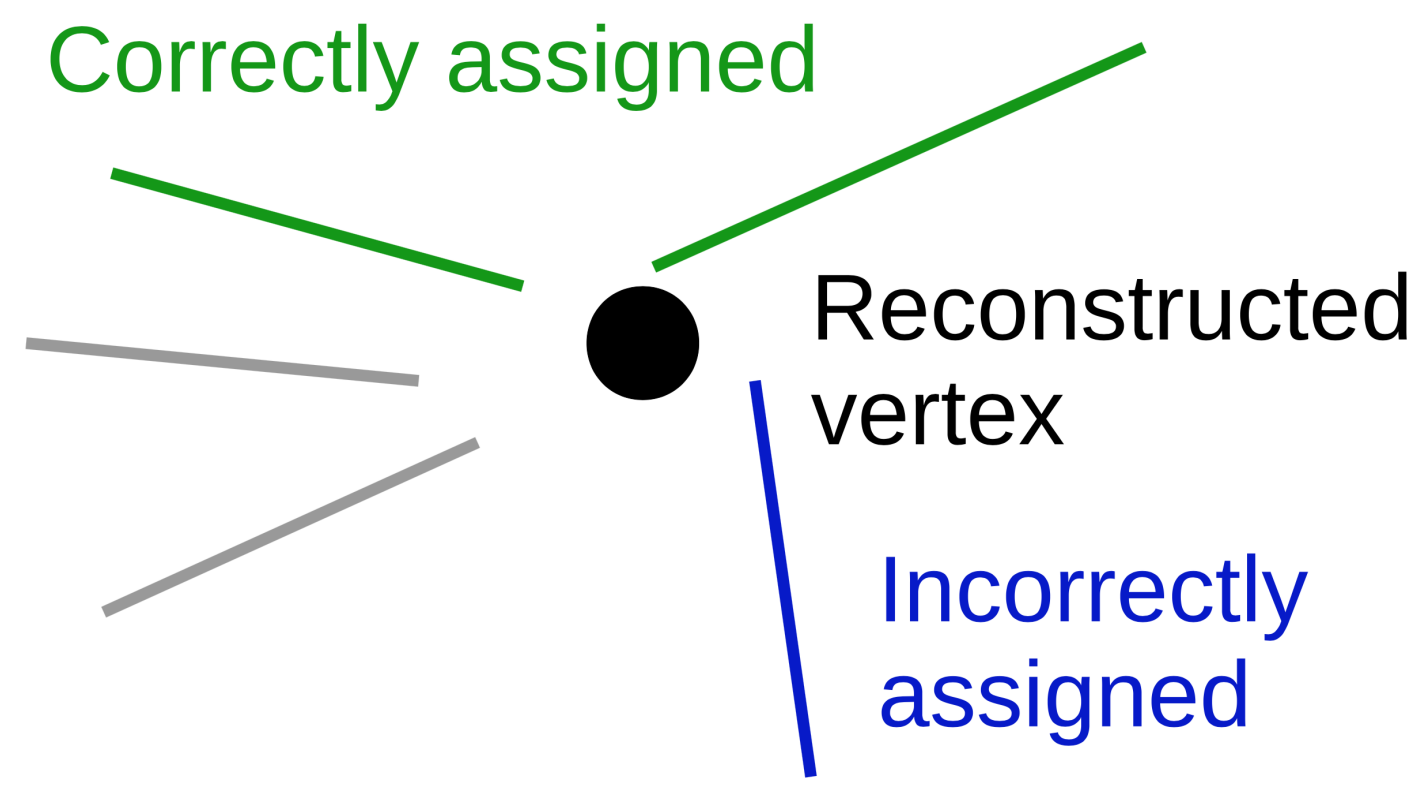

Figure 5.13: An example of reconstructed vertex cleanliness. In this example the reconstructed neutrino vertex has three tracks associated with it, however only two of them were also associated with the true neutrino vertex. In this case the reconstructed vertex has a cleanliness of $2 / 3$.

- $\boldsymbol{s}_{\text {max }}: 10-100 \mathrm{~cm}$ in $10 \mathrm{~cm}$ steps

- $\boldsymbol{b p}_{\text {max }}$ : $10-100 \mathrm{~cm}$ in $10 \mathrm{~cm}$ steps 
- $\boldsymbol{a}_{\boldsymbol{m a x}}: 5-50 \mathrm{~cm}$ in $5 \mathrm{~cm}$ steps

- $\boldsymbol{t}_{\text {vert }}: 5-50 \mathrm{~cm}$ in $5 \mathrm{~cm}$ steps

Varying these parameters simultaneously allowed the optimization process to take into account inter-variable dependencies and arrive at the most optimal permutation of parameter values in the phase-space.

The range for $s_{\max }$ was chosen to be relatively large due to the high expected uncertainty on reconstructed shower direction. As the backwards-projection of the shower is anti-collinear to the reconstructed shower direction, it also has a high uncertainty. A $100 \mathrm{~cm}$ range was chosen to allow for this uncertainty in reconstructed shower direction. The probability of a photon surviving without pair-producing decreases as it travels further from the location it was produced. As such, a photon could in theory travel the length of the TPC before producing a shower inside the TPC, however this is extremely unlikely to occur. Allowing an un-capped shower backwards-projection range when attempting to reconstruct neutrino vertices would result in contamination from cosmic showers. For this reason the range for backwards-projection, $b p_{\max }$ was capped at $100 \mathrm{~cm}$. $a_{\max }$ is also dependent on reconstructed shower direction, however only pertains to two showers that have already been associated to a common vertex. Having the additional constraint of a common vertex, a range of $50 \mathrm{~cm}$ was chosen for $a_{\text {max }}$. Signal tracks in the analysis are expected to be proton tracks from $\Delta^{+} \rightarrow p+\gamma$ decay. Protons are not MIPs and therefore the tracks they produce are relatively short, the majority being sub 100 $\mathrm{cm}$ in length as will be shown in following section. For this reason the $50 \mathrm{~cm}$ range for $t_{v e r t}$ was chosen to give coverage over the full length of the majority of proton tracks (as $t_{\text {vert }}$ is measured from both ends of the track).

Each parameter range was divided into 10 steps. The primary limit on the parameter phase-space resolution was computing time and with 10 steps for each of the parameters, the vertex algorithm had to be run $10^{4}$ times over the NC $\Delta$ radiative + cosmics and BNB + cosmics samples. Subsequent scans were performed over a shorter parameter ranges around the parameter regions of interest produced by the initial parameter phase-space scan. Subsequent scans wer also performed over larger parameter ranges to ensure the appropriate region of parameter phase-space had been covered by the initial scan. While varying the four shower-related parameters, $t_{\max }$ was held at $4 \mathrm{~cm}$. Typically the distance between tracks sharing a common vertex is sub $1 \mathrm{~cm}$ and the tracks appear connected at the vertex. There are regions of unresponsive wires in the MicroBooNE TPC, however, which do not allow for the observation of ionisation electrons in those regions. On average the larger wire 
gaps tends to be 13-15 consecutive wires wide on a plane [80]. Given the inter-wire spacing in MicroBooNE is $3 \mathrm{~mm}$, the maximum track association start/end point proximity, $t_{\max }$ was chosen to be $14 \times 0.3 \approx 4 \mathrm{~cm}$.

The vertex algorithm was run on the full NC $\Delta$ radiative + cosmics and $\mathrm{BNB}+$ cosmics samples for each parameter permutation and $c p q$ was calculated for all of the reco-vertices produced by that permutation. The parameter permutation that produced the largest fraction of reco-vertices with $c p q=1$ for a sample was taken to be the "best" permutation for that sample. Two criteria were placed on the vertices for optimization. The first required vertices to be from events where the true neutrino vertex was contained inside the TPC. The second required at least one reco-shower associated with the reco-vertex to be truly associated with the true neutrino vertex, ensuring no cosmic-only reco-vertices contaminated the results. The parameters optimized for each sample and the corresponding percentage of vertices with $c p q=1$ can be seen in Table 5.1 .

\begin{tabular}{|c|c|c|c|c|c|c|}
\hline Sample & $c p q=1 \%$ & $t_{\max }[\mathrm{cm}]$ & $s_{\max }[\mathrm{cm}]$ & $b p_{\max }[\mathrm{cm}]$ & $a_{\max }[\mathrm{cm}]$ & $t_{\text {vert }}[\mathrm{cm}]$ \\
\hline NCDR (NCDR optimized) & 80.2 & 4 & 30 & 100 & 25 & 5 \\
\hline NCDR (BNB optimized) & 76.3 & 4 & 70 & 70 & 30 & 30 \\
\hline BNB (NCDR optimized) & 62.3 & 4 & 30 & 100 & 25 & 5 \\
\hline BNB (BNB optimized) & 63.5 & 4 & 70 & 70 & 30 & 30 \\
\hline
\end{tabular}

Table 5.1: Performance of the vertex algorithm on the BNB + cosmic (BNB) and NC $\Delta$ radiative (NCDR) samples using optimized parameters on both samples. Listed are the sample and the choice of optimized parameters, the percentage of reco-vertices for a given parameter permutation with $c p q=1$ and the parameter values themselves.

The NCDR sample using corresponding NCDR optimized parameters results in approximately $80 \%$ of its vertices $c p q=1$, whereas the BNB sample using BNB optimized parameters results in approximately $63 \%$ of vertices with $c p q=1$. This implies that NC $\Delta$ radiative decay is an easier shower-producing interaction to reconstruct compared to a typical BNB shower-related interaction. This is expected given the comparatively simple single shower topologies produced by NC $\Delta$ radiative decay: $\Delta^{0} \rightarrow n+\gamma$ which produces a lone shower and $\Delta^{+} \rightarrow p+\gamma$ which produces a single shower and single track. A typical shower producing BNB interaction is $\pi^{0} \rightarrow 2 \gamma$, a much harder topology to correctly reconstruct due to the presence of two showers that must be properly reconstructed and associated through backwardsprojection. The sample-optimized values for $s_{\max }$ of $30 \mathrm{~cm}$ and $70 \mathrm{~cm}$ for the NC $\Delta$ radiative + cosmic and $\mathrm{BNB}+$ cosmic samples respectively demonstrate the high uncertainty on shower direction (this is also indicative of the greater difficulty in correctly associating two showers together in the case of the BNB optimized param- 
eters). Given the higher overall performance of the NCDR optimized parameters $(80.2 \%$ and $62.3 \%)$ versus the BNB optimized parameters $(76.3 \%$ and $63.5 \%)$ and the importance of NC $\Delta$ radiative decay as the signal, the NCDR optimized parameters were chosen for the analysis.

Figure 5.14 shows the performance of the vertex reconstruction algorithm for the NC $\Delta$ radiative + cosmics sample using NCDR optimized parameters, in terms of the performance quantities: completeness, cleanliness and $c p q$. Each of the four plots shows one of the parameters that were varied during optimization. Also included are the performance quantities for standard Pandora vertex reconstruction. Using the vertex reconstruction algorithm on this sample with corresponding optimized parameters shows much better performance than Pandora in terms of cleanliness and $c p q$ but marginally worse performance in terms of completeness. As was mentioned in Section 4.2, if no association can be found for a shower, Pandora will by default associated that shower with the candidate neutrino vertex. This can result in the association of cosmic showers with the candidate neutrino vertex, giving Pandora vertices relatively high levels of completeness but lower levels of cleanliness. The vertex algorithm used in this analysis does not perform such a default shower association and therefore has a higher cleanliness than Pandora. As NC $\Delta$ radiative produces a relatively simple single shower topology, the loss in completeness for the vertex reconstruction algorithm that results from not performing the same default shower association as Pandora is minimal. Performance for the NC $\Delta$ radiative + cosmics sample is much more dependent on $s_{\max }$ and $b p_{\max }$ than $a_{\max }$ and $t_{\text {vert }}$. Increasing the value of $s_{\max }$ and $b p_{\max }$ causes the ratio of good vertices for completeness to increase and the ratio of good vertices for cleanliness to decrease. Increasing these parameters will allow more reco-tracks and reco-showers that are further away and have a larger impact parameter to be associated with reco-vertices, thus increasing the number of correctly and incorrectly associated reco-tracks and reco-showers. This consequently increases completeness and reduces cleanliness.

Figure 5.15 shows the performance of the vertex reconstruction algorithm for the BNB + cosmics sample using BNB optimized parameters, displayed in the same manner as the NC $\Delta$ radiative + cosmics sample in Figure 5.14. Using the vertex reconstruction algorithm on this sample with corresponding optimized parameters shows better performance than Pandora in terms of cleanliness and $c p q$ but moderately worse performance in terms of completeness. This behaviour can again be explained by the default shower association performed by Pandora if no other association is found. The difference in completeness between the vertex reconstruction algorithm and Pandora is much more significant for the BNB + cosmics sample. Un- 

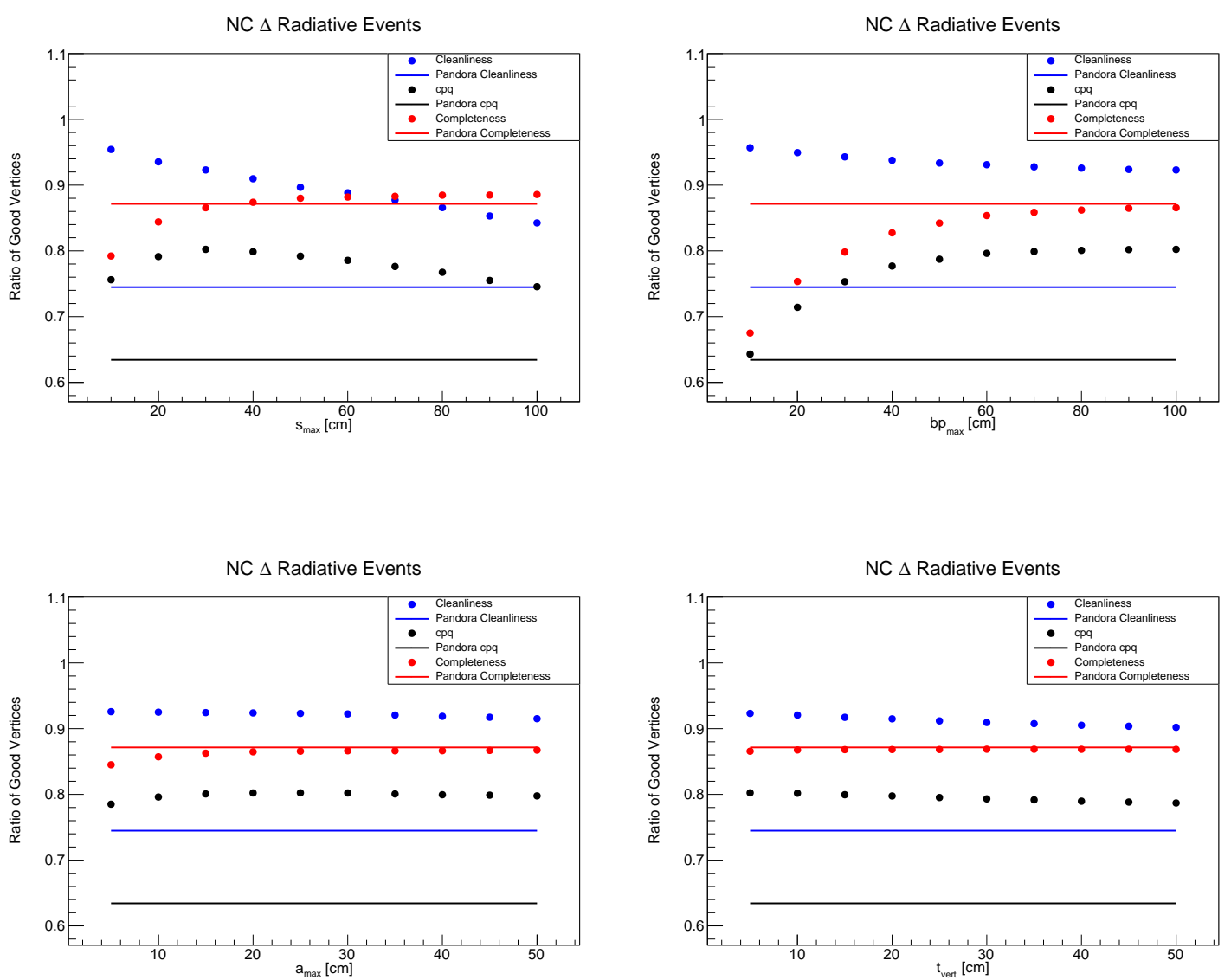

Figure 5.14: Plots for the NC $\Delta$ radiative + cosmics sample showing the change in vertex completeness, cleanliness and $c p q$ as each of the input vertex reconstruction algorithm parameters is varied. For each plot the parameter on the $x$-axis is varied while all other parameters are held at the NCDR optimized values shown in Table 5.1. The $y$-axis shows the ratio of good vertices: the ratio of vertices that have a performance quantity (completeness, cleanliness or $c p q$ ) equal to one. Also included are lines dictating the ratio of good vertices for completeness, cleanliness and $c p q$ using standard Pandora vertex reconstruction.

like the relatively simple NC $\Delta$ radiative single shower topology, multiple processes in the BNB + cosmic sample will produce $\pi^{0}$ s that produce two photon showers. Such multiple shower topologies are more difficult to reconstruct (particularly if there is no visible activity attached to the vertex) than single shower topologies and so the vertex reconstruction algorithm will miss more of these showers compared to the NC $\Delta$ radiative + cosmics sample. Pandora's default shower association removes the loss of unassociated $\pi^{0}$ showers and so the completeness comparison between Pandora and the vertex reconstruction algorithm is much more favourable for the $\mathrm{BNB}+$ cosmics sample than the $\mathrm{NC} \Delta$ radiative + cosmics sample.

Additional multi-parameter scans were performed over much shorter ranges centered around the best parameter from the initial results shown in Table 5.1, to give a higher resolution search in these areas. As the optimized values for the NC $\Delta$ 

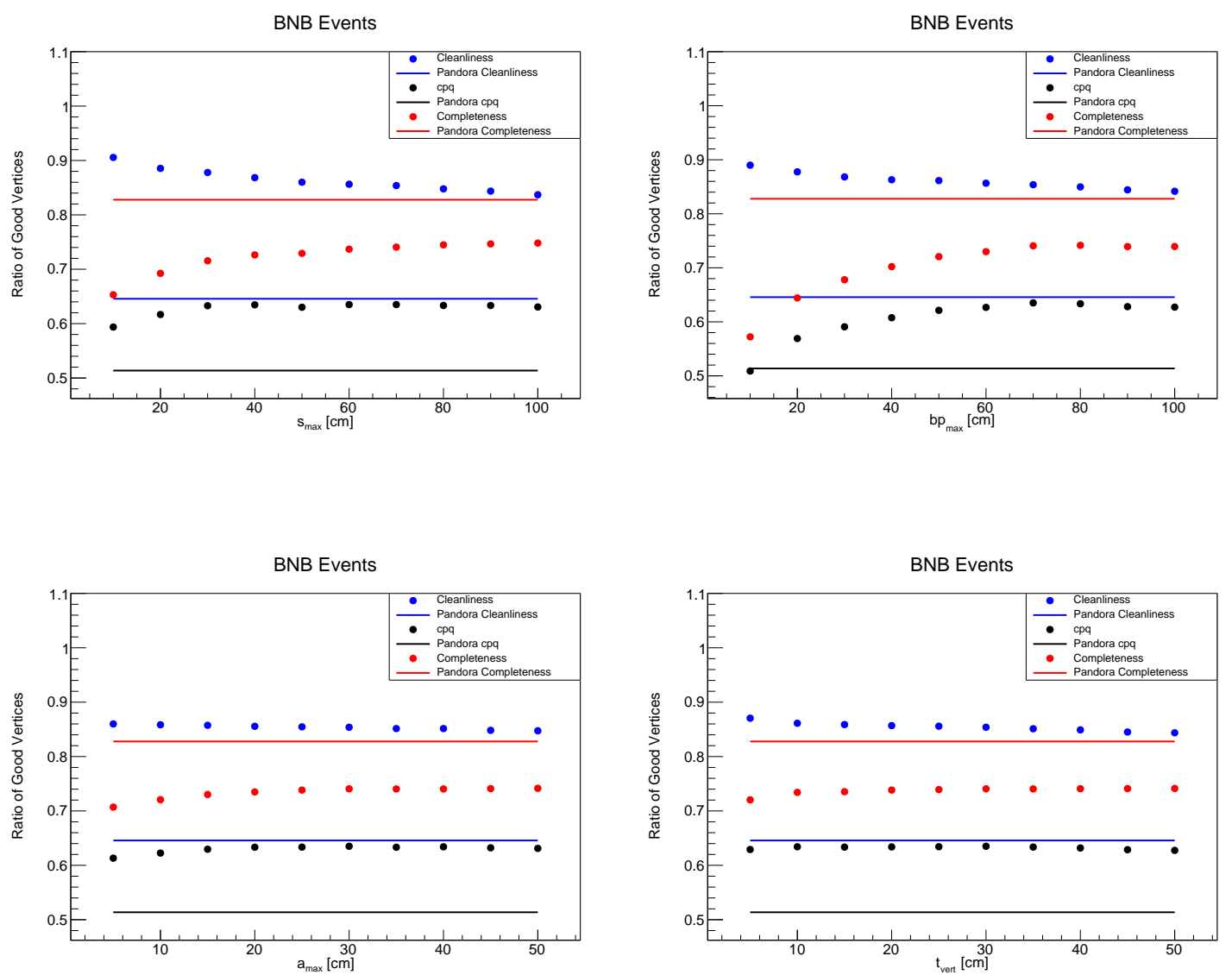

Figure 5.15: Plots for the BNB + cosmics sample, displayed in the same manner is Figure 5.14 but for the BNB optimized parameters shown in Table 5.1 .

radiative + cosmics and $\mathrm{BNB}+$ cosmics sample are different, the ranges chosen for each sample scan were different. The higher resolution parameter ranges for the NC $\Delta$ radiative + cosmics sample were:

- $\boldsymbol{s}_{\max }: 20-40 \mathrm{~cm}$ in $2 \mathrm{~cm}$ steps

- $\boldsymbol{b p}_{\boldsymbol{m a x}}: 90-110 \mathrm{~cm}$ in $2 \mathrm{~cm}$ steps

- $\boldsymbol{a}_{\boldsymbol{m a x}}: 20-30 \mathrm{~cm}$ in $1 \mathrm{~cm}$ steps

- $\boldsymbol{t}_{\text {vert }}: 1-11 \mathrm{~cm}$ in $1 \mathrm{~cm}$ steps

These ranges were centered around the NCDR optimized parameters shown in Table $5.1\left(s_{\max }: 30 \mathrm{~cm}, b p_{\max }: 100 \mathrm{~cm}, a_{\max }: 25 \mathrm{~cm}, t_{v e r t}: 5 \mathrm{~cm}\right)$ with the exception of $t_{\text {vert }}$ range, which was shifted upwards by $1 \mathrm{~cm}$ to ignore the $0 \mathrm{~cm} t_{\text {vert }}$ value which would have otherwise been included in the range. Figure 5.16 shows the performance of the vertex reconstruction algorithm for the NC $\Delta$ radiative + cosmics sample in the same manner as Figure 5.14, but over the higher resolution NC $\Delta$ radiative + cosmics parameter ranges listed above. The change in ratio of vertices with $c p q=1$ as each of the parameters is varied over these higher resolution ranges is extremely 
small, indicating that further study into more precise optimized parameters for the $\mathrm{NC} \Delta$ radiative + cosmics sample would be redundant.
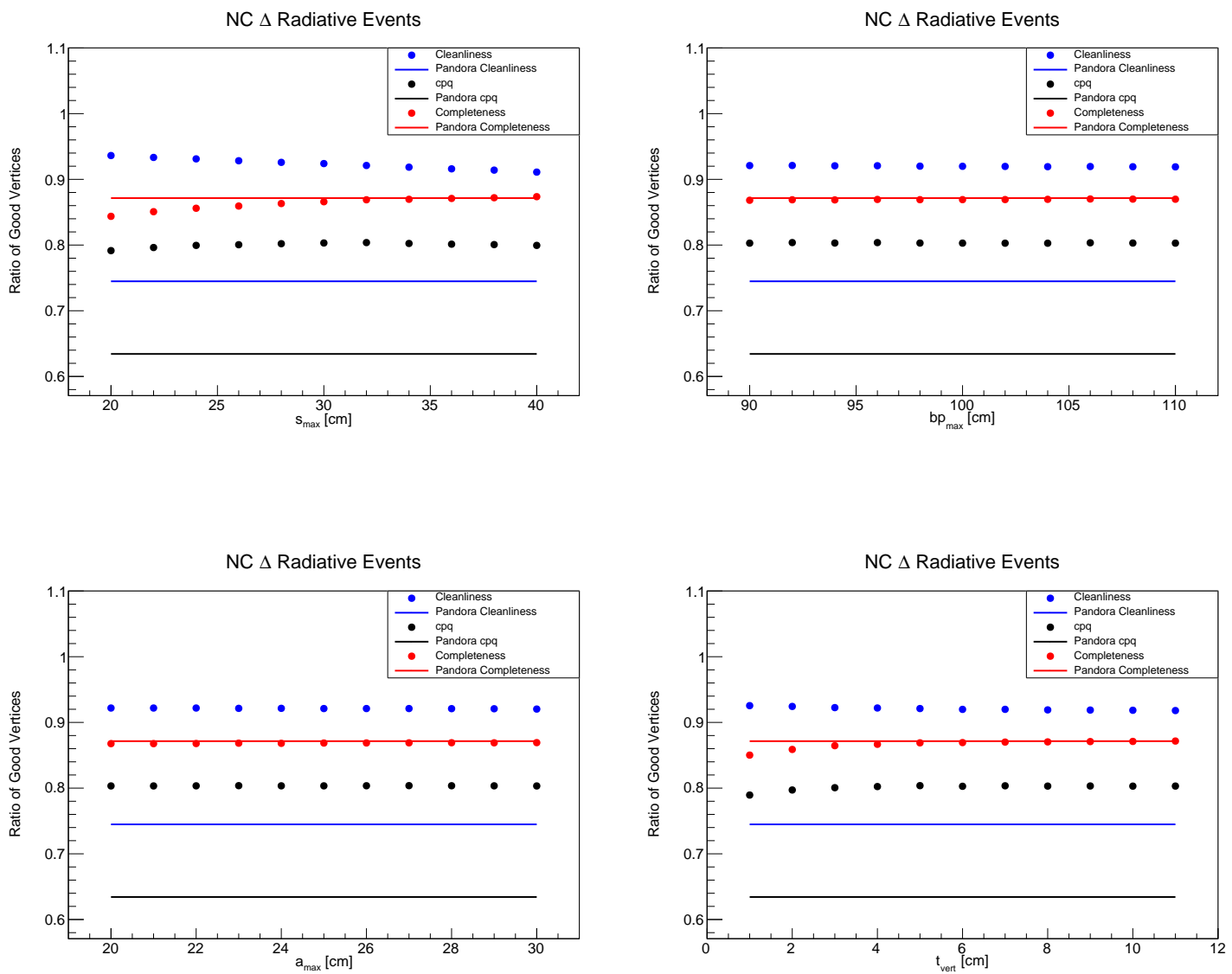

Figure 5.16: Plots for the NC $\Delta$ radiative + cosmics sample showing the change in vertex completeness, cleanliness and $c p q$ as each of the input vertex reconstruction algorithm parameters is varied. Displayed in the same manner is Figure 5.14 . but over much shorter ranges centered around the best parameters from the initial NCDR optimized results shown in Table 5.1.

The higher resolution parameter ranges for the BNB + cosmics sample were:

- $\boldsymbol{s}_{\text {max }}: 60-80 \mathrm{~cm}$ in $2 \mathrm{~cm}$ steps

- $\boldsymbol{b}_{\text {max }}: 60-80 \mathrm{~cm}$ in $2 \mathrm{~cm}$ steps

- $\boldsymbol{a}_{\text {max }}: 25-35 \mathrm{~cm}$ in $1 \mathrm{~cm}$ steps

- $\boldsymbol{t}_{\text {vert }}: 25-35 \mathrm{~cm}$ in $1 \mathrm{~cm}$ steps

These ranges were centered around the BNB optimized parameters shown in Table 5.1 ( $\left.s_{\max }: 70 \mathrm{~cm}, b p_{\max }: 70 \mathrm{~cm}, a_{\max }: 30 \mathrm{~cm}, t_{\text {vert }}: 30 \mathrm{~cm}\right)$. Figure 5.17 shows the performance of the vertex reconstruction algorithm for the BNB + cosmics sample in the same manner as Figure 5.15, but over the higher resolution BNB + 
cosmics parameter ranges listed above. Similarly to the higher resolution multiparameter scan for $\mathrm{NC} \Delta$ radiative + cosmics sample, the change in ratio of vertices with $c p q=1$ as each of the parameters is varied over the higher resolution ranges is extremely small. This indicates that further study into more precise optimized parameters for the BNB + cosmics sample would also be redundant.
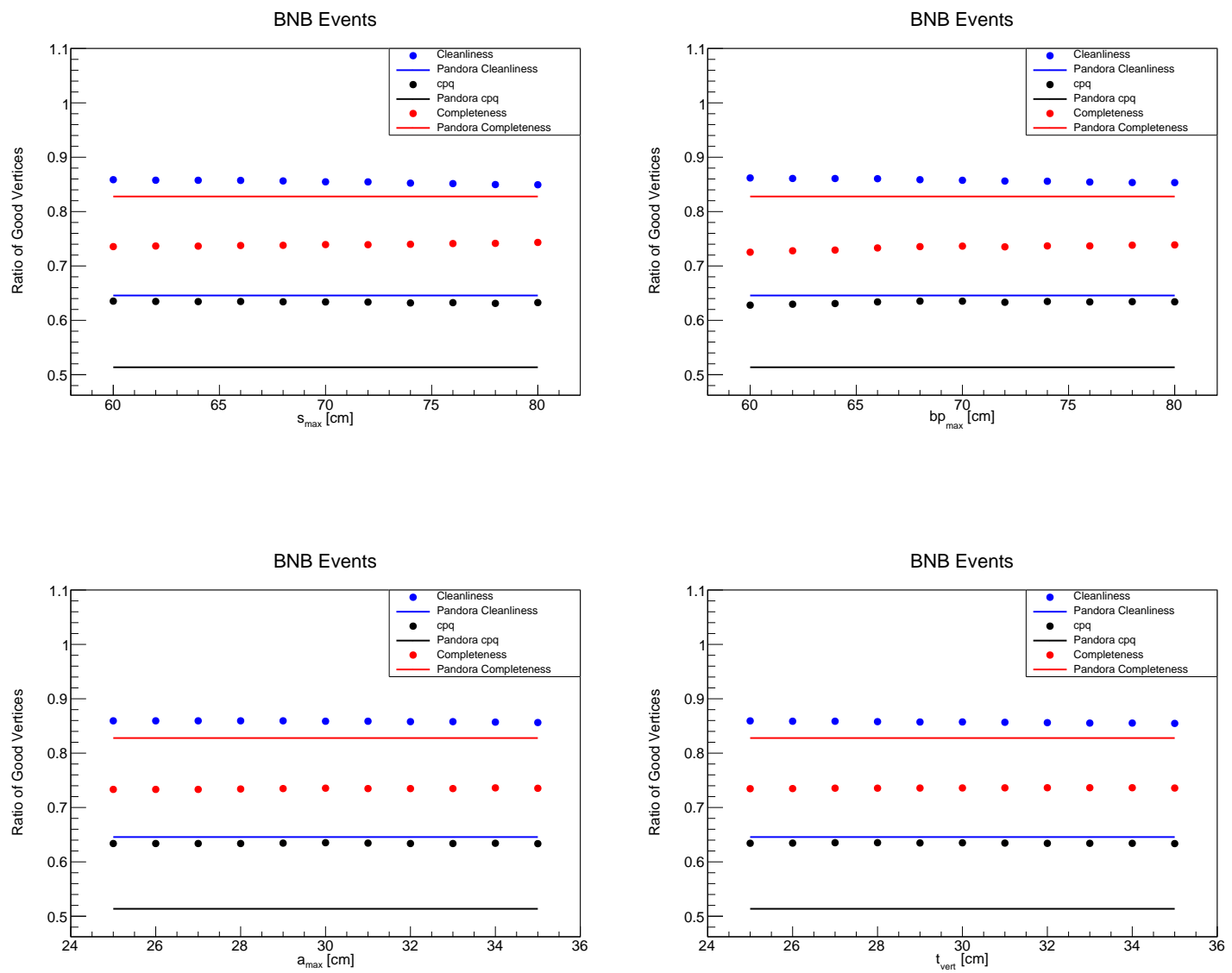

Figure 5.17: Plots for the BNB + cosmics sample showing the change in vertex completeness, cleanliness and $c p q$ as each of the input vertex reconstruction algorithm parameters is varied. Displayed in the same manner is Figure 5.14 , but over much shorter ranges centered around the best parameters from the initial BNB optimized results shown in Table 5.1 .

Additional multi-parameter scans were also performed over extended parameter ranges to ensure the appropriate region of parameter phase-space was covered for each sample. For these scans, the range of each parameter was doubled compared to the initial parameter ranges, while the number of steps remained the same:

- $\boldsymbol{s}_{\max }: 20-200 \mathrm{~cm}$ in $10 \mathrm{~cm}$ steps

- $\boldsymbol{b} \boldsymbol{p}_{\max }: 20-200 \mathrm{~cm}$ in $10 \mathrm{~cm}$ steps

- $\boldsymbol{a}_{\text {max }}: 10-100 \mathrm{~cm}$ in $5 \mathrm{~cm}$ steps 
- $\boldsymbol{t}_{\text {vert }}: 10-100 \mathrm{~cm}$ in $5 \mathrm{~cm}$ steps
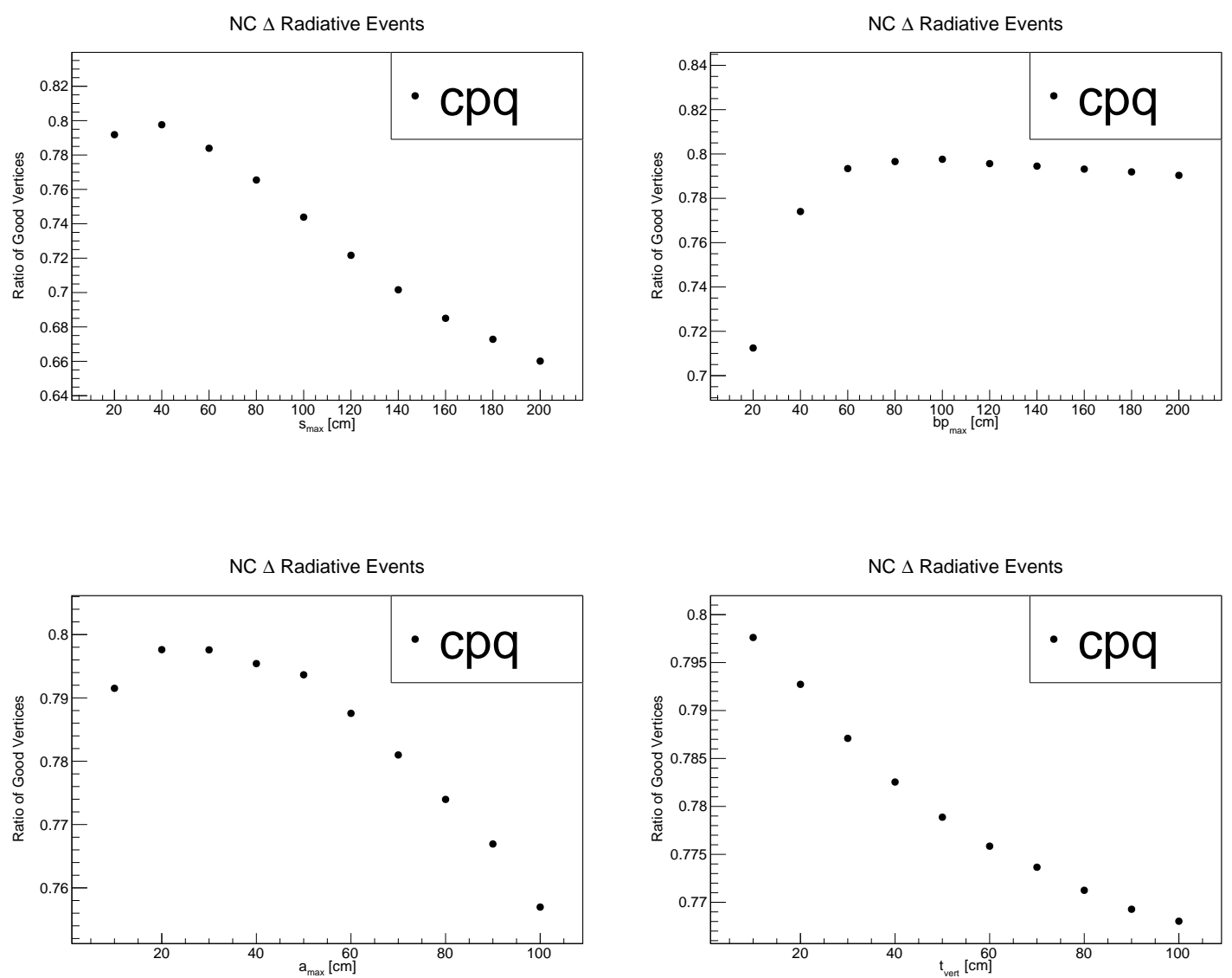

Figure 5.18: Plots for the NC $\Delta$ radiative + cosmics sample showing the change in vertex $c p q$ for each parameter over extended parameter ranges.

Figure 5.18 shows the performance of the vertex reconstruction algorithm for the NC $\Delta$ radiative + cosmics sample for the extended parameter ranges listed above. In order to better display the changes in $c p q$ over the extended parameter ranges, Figure 5.18 includes only the ratio of vertices with $c p q=1$ and the $y$-axis of each parameter plot is magnified. For each parameter, a peak can be seen in the same regions as the optimized parameters selected by the initial $\mathrm{NC} \Delta$ radiative + cosmics multi-parameter scan (the NCDR optimized parameters in Table 5.1). This indicates that the initial multi-parameter scan ranges were performed over the correct parameter ranges for the $\mathrm{NC} \Delta$ radiative + cosmics sample.

Figure 5.19 shows the performance of the vertex reconstruction algorithm for the $\mathrm{BNB}+$ cosmics sample for the same extended parameter ranges that were used for the NC $\Delta$ radiative + cosmics sample. For each parameter, a peak can be seen in the same regions as the optimized parameters selected by the initial BNB + cosmics multi-parameter scan (the BNB optimized parameters in Table 5.1). As for the NC $\Delta$ radiative + cosmics sample, this indicates that the initial multi-parameter scans 

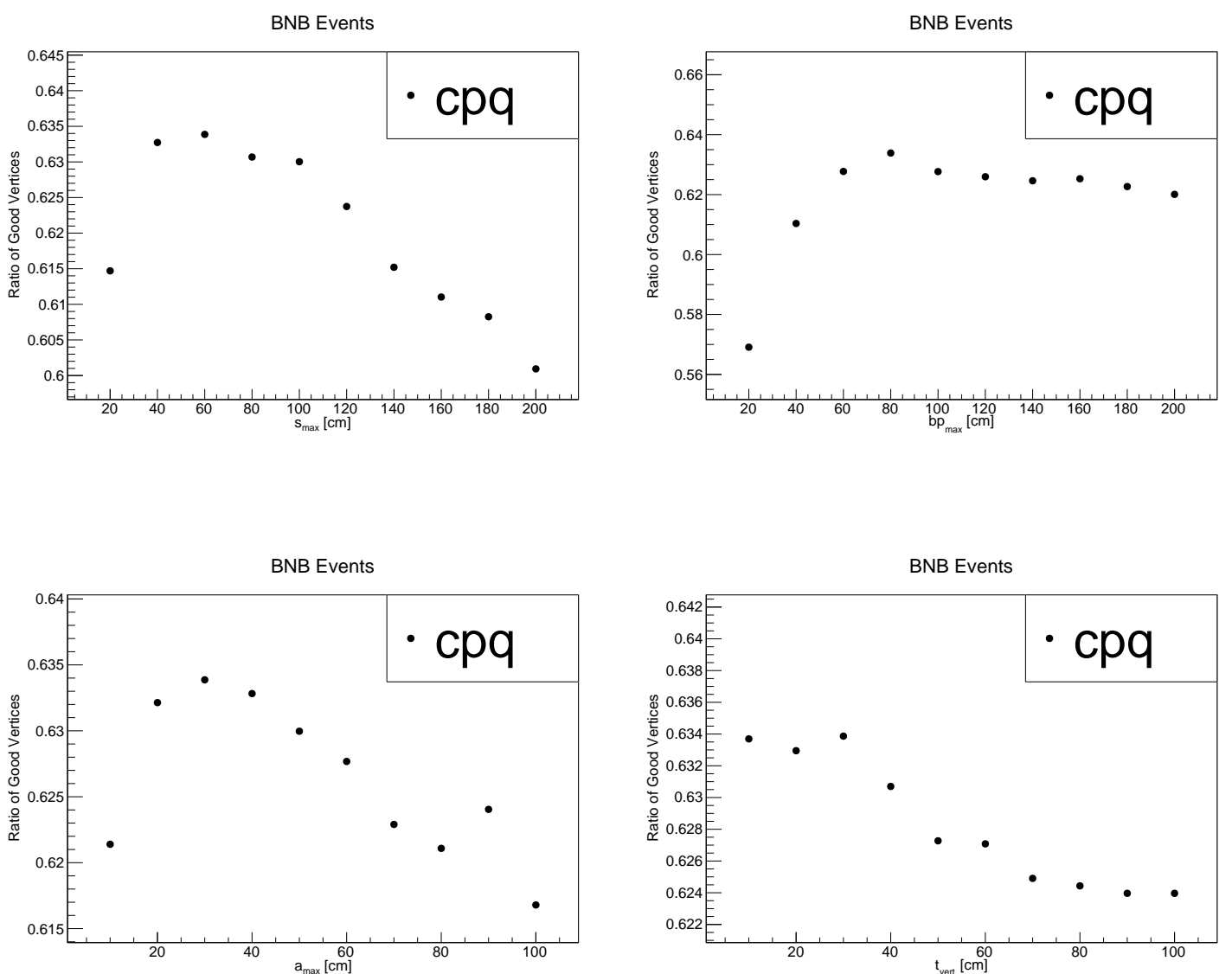

Figure 5.19: Plots for the BNB + cosmics sample showing the change in vertex $c p q$ for each parameter over extended parameter ranges.

were performed over the correct parameter ranges for the BNB + cosmics sample. The extended parameter plots for the BNB + cosmics sample are more irregular than their NC $\Delta$ radiative + cosmics counterparts. This can be attributed to the fact that the NC $\Delta$ radiative + cosmics sample contains only $\mathrm{NC} \Delta \rightarrow N+\gamma$, whereas the BNB + cosmics sample contains all of the neutrino interactions expected in MicroBooNE. As such, changes to the parameters will potentially affect the vertex algorithm performance of different interactions in different ways, resulting in more irregular changes for the overall performance of the BNB + cosmics sample.

As the choice was made to use NCDR optimized parameters for the analysis, the behaviour of this parameter choice on the BNB + cosmics sample is also of interest. Figure 5.20 shows the same performance plots as Figure 5.14 but for the BNB + cosmics sample using NCDR optimized parameters. Comparing each of the parameter plots to the BNB + cosmics sample parameter plots using BNB optimized parameters (Figure 5.15) shows that there is very little difference in performance in using the NCDR or BNB optimized parameters on the BNB + cosmics sample. This is to be expected given the relatively small difference in $c p q$ performance between 
the two parameter choices as shown in Table 5.1 (62.3\% of vertices with $c p q=1$ for NCDR optimized vs $62.3 \%$ of vertices with $c p q=1$ for BNB optimized). The lower completeness and higher cleanliness of the vertex reconstruction algorithm compared to Pandora seen in Figure 5.15 is also seen here.
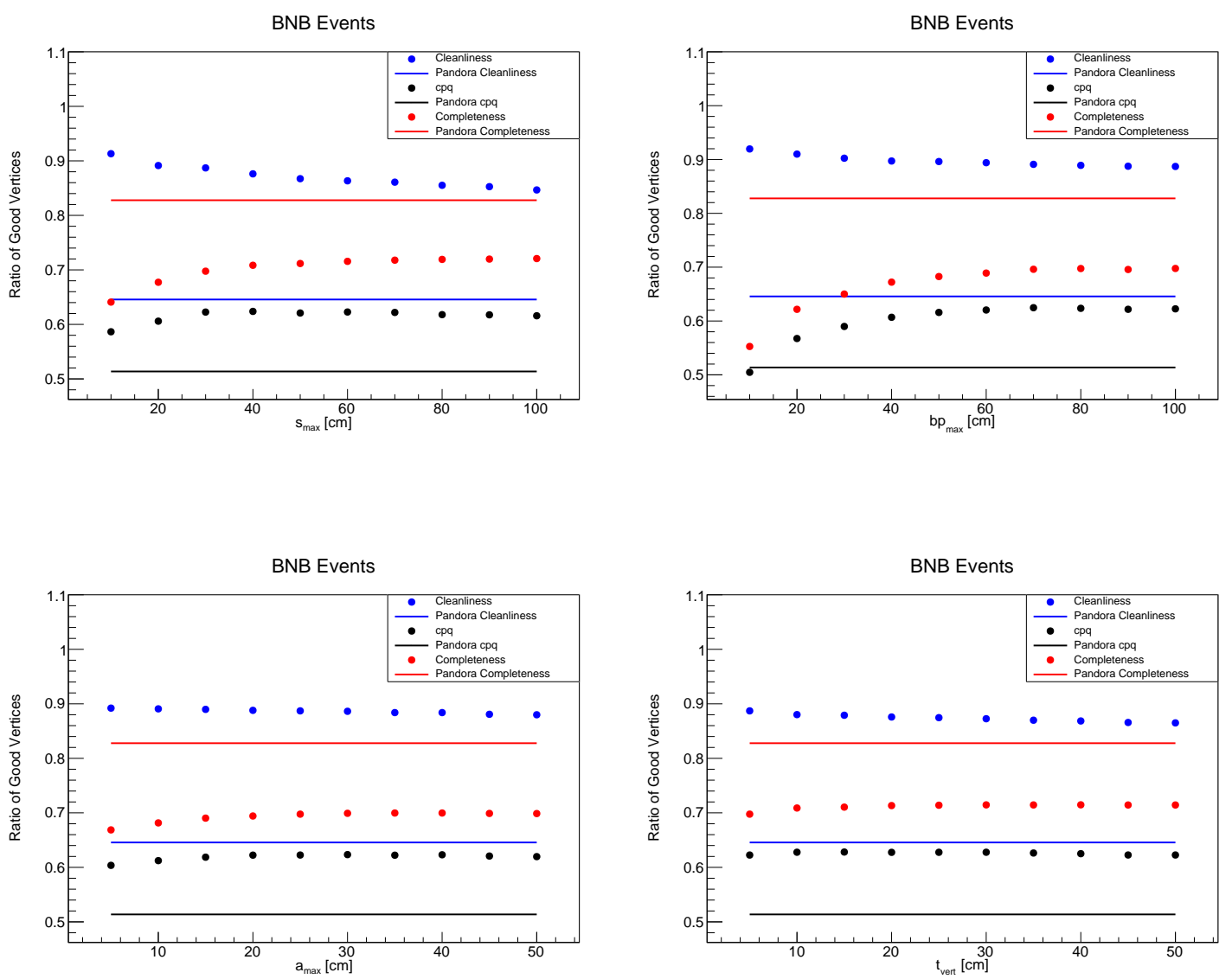

Figure 5.20: Plots for the BNB + cosmics sample, displayed in the same manner is Figure 5.14 but for the NCDR optimized parameters shown in Table 5.1.

Figure 5.21 shows the vertex position resolution of the $\mathrm{NC} \Delta$ radiative + cosmics sample using NCDR optimized parameters for the vertex reconstruction algorithm. Considering the 3-d distance between the true and reconstructed vertices (top left plot), both methods appear to perform similarly at lower distances but the mean values for each method show that Pandora has, on average, a larger number of failures at larger distances. There is a noticeable offset for the reco-true vertex distance in the $x$-axis for both methods (top right plot). This is a result of space-charge effects altering the drift electric field, drift time of ionisation electrons and thus the reconstructed positions of tracks and showers in the $x$-axis.

Figure 5.22 shows the vertex position resolution of the vertex reconstruction algorithm for the BNB + cosmics sample using NCDR optimized parameters. The 

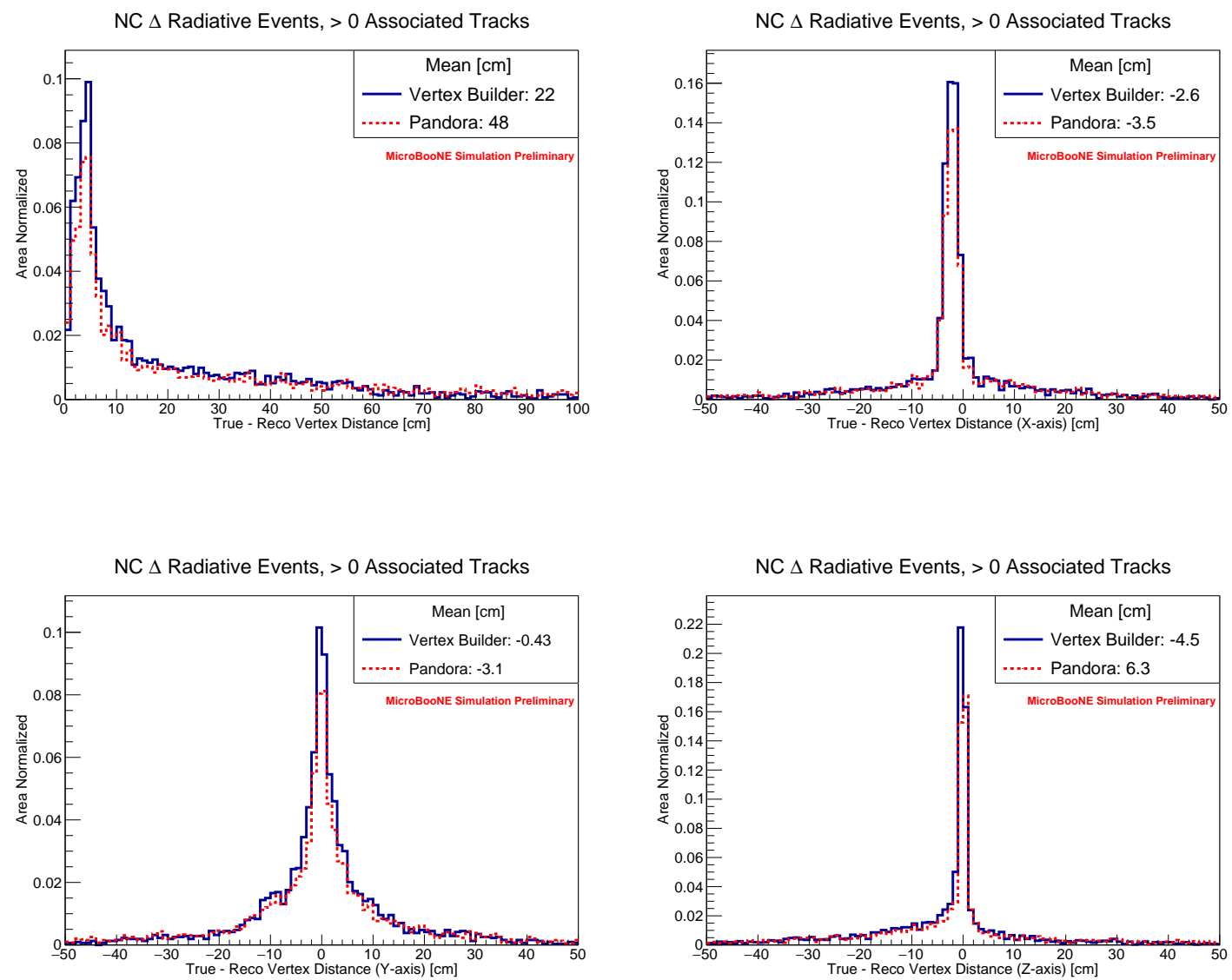

Figure 5.21: Plots for the NC $\Delta$ radiative + cosmics sample using NCDR optimized parameters showing the vertex position resolution. Includes only vertices with at least one associated track. Top left: 3-d true-neutrino vertex - reconstructed vertex distance, top right: $x$-axis distance, bottom left: $y$-axis distance, bottom right: $z$ axis distance. Plotted for the vertex reconstruction algorithm and standard Pandora vertex reconstruction, both area normalized.

performance of the vertex reconstruction algorithm and Pandora is very similar to the NC $\Delta$ radiative + cosmics sample. The offset in $x$ seen in the NC $\Delta$ radiative + cosmics vertex position resolution is also present here. 

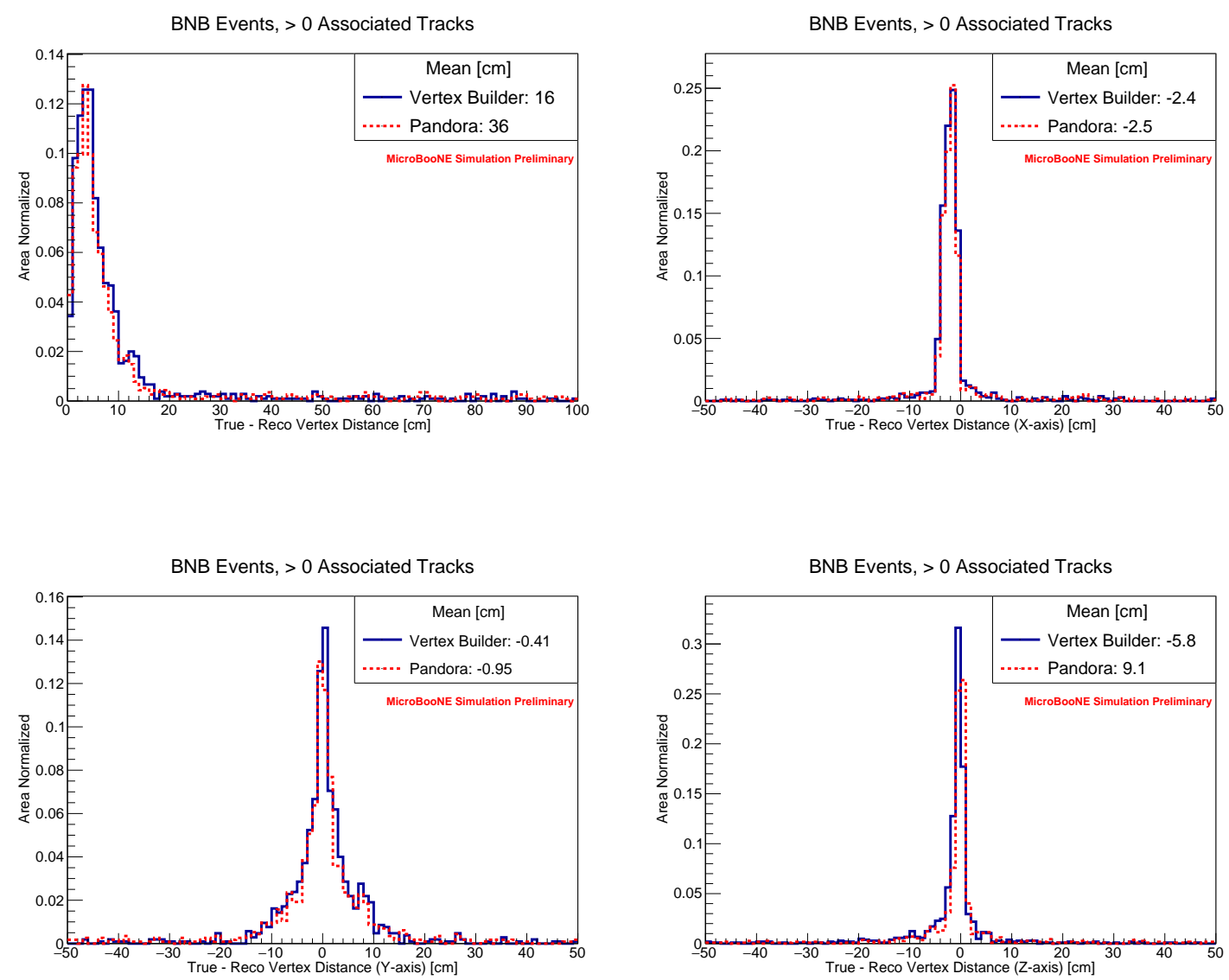

Figure 5.22: Same as the plots in Figure 5.21 but for the BNB + cosmics sample (using NCDR optimized parameters). 


\subsection{Neutral Current $\Delta$ Radiative Topologies}

The ultimate goal of the analysis is to search for a photon-based LEE in MicroBooNE operating under the assumption that the LEE in MiniBooNE was the result of a mis-estimation of the MiniBooNE LEE NC photon background. Specifically the analysis assumes the source of the MiniBooNE LEE is an enhanced rate of NC $\Delta$ radiative decay, one of the most significant photon backgrounds to the MiniBooNE LEE. The signature of the MiniBooNE LEE (and also the NC $\Delta$ radiative LEE background) is a single shower, thus this analysis focuses on reco-vertices produced by the vertex reconstruction algorithm with a single associated shower. Two NC $\Delta$ radiative topologies are considered in the analysis:

- $1 \gamma \mathbf{0 p}$ : Produced by $\Delta^{0} \rightarrow n+\gamma$ decay, where the photon will produce a single shower and the neutron will remain undetected.

- $1 \gamma \mathbf{1} p$ : Produced by $\Delta^{+} \rightarrow p+\gamma$ decay, where the photon will produce a single shower. While the proton produced in this interaction would typically not be energetic enough to breach the Cherenkov threshold in MiniBooNE, it will have enough energy to ionise the liquid argon in MicroBooNE and will therefore be observable as a short track.

FSIs make it is possible for extra tracks to be produced in a NC $\Delta$ radiative interaction that would constitute a $1 \gamma N p$ sample where $N>1$ (a vertex with a single shower and multiple tracks). This topology accounts for less than $10 \%$ of $\Delta$ radiative events and is typically rejected as background by the event selection process. For this reason this topology is not considered in the analysis.

\subsection{Monte Carlo and Data Samples}

The following describes all reconstructed vertex samples used for the analysis. These are samples produced by running the vertex reconstruction algorithm on simulated and data events in MicroBooNE. All MC BNB neutrino interactions are generated using GENIE and all MC cosmics are generated using CORSIKA. The first three samples are the signal and background prediction samples used for the analysis:

- NC $\Delta$ radiative + cosmics: vertices reconstructed from MC BNB NC $\Delta$ radiative events. To classify as signal the radiatively produced photon must exit the nucleus. Any number of protons or neutrons produced by the interaction may exit the nucleus but no other particles are permitted. This sample was produced by generating BNB NC resonant events and selecting the events that fit the signal definition. CORSIKA cosmics are overlaid on 
each NC $\Delta$ radiative event. The true neutrino interactions were generated in the TPC volume only. This sample contains reconstructed signal vertices and reconstructed cosmic background vertices from the overlaid simulated cosmic activity. This is the signal prediction for the analysis (plus background from cosmic activity in the same BNB signal event).

- BNB + cosmics: vertices reconstructed from MC BNB inclusive neutrinoargon interactions with overlaid CORSIKA cosmics. The true neutrino interactions were generated over the entire liquid argon volume contained in the cryostat. This sample contains reconstructed BNB background vertices and reconstructed cosmic background vertices from the simulated overlaid cosmic activity. This is the BNB related background prediction sample for the analysis (plus background from cosmic activity in the same BNB background event).

- BNB external cosmic data: vertices reconstructed from cosmic data recorded outside of the BNB beam spills. As was mentioned in Subsection 3.2 .2 , the probability that a BNB beam-spill producing a neutrino interaction in the MicroBooNE detector is approximately 1/600. If cosmic activity produces light sufficient to activate the PMT trigger (see Subsection 3.2.2) in-time with a beam spill where no neutrino interactions occurs (i.e. 599/600 of the time a beam-spill is present) then the BNB hardware trigger (see Subsection 3.2.2 will also activate and this cosmic activity with no neutrino interaction will be erroneously recorded as a BNB event. Such "BNB mimicking cosmic events" occur extremely frequently and are a large background to any BNB-based analysis. BNB external cosmic data is taken using the BNB external hardware trigger which guarantees a $\mathrm{BNB}$ beam-spill is not present in the detector (i.e. the opposite of the BNB hardware trigger). When the BNB external hardware trigger and the PMT trigger are activated any cosmic activity in the detector will be recorded in a BNB external cosmic data event. These events should perfectly emulate the BNB mimicking cosmic background taken with the BNB hardware trigger, making them a powerful tool for characterising this background. The BNB external cosmic data sample is used as the prediction for the BNB mimicking cosmic background in the analysis.

While there are three different cosmic contributions from each of the three samples used in the analysis, this is not a case of double-counting. The overlaid cosmics of the BNB signal and background samples are mutually exclusive from the BNB mimicking cosmics, as the former are only present with a BNB neutrino interaction whereas the latter are present when no BNB neutrino interaction is produced by the beam-spill. The remaining samples are used either to train event selection or for data-MC validation: 
- $\mathrm{NC} \Delta$ radiative: vertices filtered from the NC $\Delta$ radiative + cosmics sample. Only reco-vertices whose associated reco-objects are all associated with the true NC $\Delta$ radiative interaction vertex are selected for this sample. This removes any reco-vertices contaminated by cosmics. This is a pure signal sample used to train the event selection.

- BNB: vertices filtered from the BNB + cosmics sample. Only reco-vertices whose associated reco-objects are all associated with the true neutrino interaction are accepted for this sample. This is a pure BNB background sample used to train the event selection.

- In-time cosmics: vertices reconstructed from a sample of CORSIKA cosmics that are in time with the beam (with no BNB interactions). The in-time cosmics sample is essentially a simulated sample of BNB mimicking cosmic background and consists only of simulated cosmics that produce light in-time with the beam. While this sample could be used to characterise the BNB mimicking cosmic background, the BNB external cosmic data sample is a much more reliable choice as it does not depend on simulation, thus the intime cosmics sample is only used to train the event selection.

- Unblinded data: vertices reconstructed from the $4.8 \times 10^{19}$ POT unblinded MicroBooNE BNB data taken using the BNB hardware trigger. Assuming the MicroBooNE simulations are correct this data can be characterised by combining and appropriately scaling the three prediction samples: $\mathrm{NC} \Delta$ radiative + cosmics, BNB + cosmics and BNB external cosmic data. The unblinded data sample is used for cross-checks and data-MC comparisons and is a smaller version of the $6.6 \times 10^{20}$ POT data sample the estimates for the analysis in this thesis are based on.

Table 5.2 contains the event, POT and reconstructed vertex numbers for each of the samples.

\begin{tabular}{|l|l|l|l|}
\hline Sample & Events & POT & Vertices \\
\hline NC $\Delta$ radiative + cosmics & 350,000 & $1.7 \times 10^{24}$ & 480,000 \\
\hline BNB + cosmics & $2,100,000$ & $2.2 \times 10^{21}$ & $4,400,000$ \\
\hline In-time cosmics & 990,000 & - & $1,200,000$ \\
\hline BNB external cosmic data & 850,000 & - & 850,000 \\
\hline Unblinded data: & 190,000 & $4.8 \times 10^{19}$ & 180,000 \\
\hline
\end{tabular}

Table 5.2: A table of generated event samples and data used to produce the recovertex samples. Number of generated events, corresponding POT and the number of reco-vertices produced by the vertex reconstruction algorithm are listed. 


\subsection{Pre-selection}

Following vertex reconstruction, a series of pre-selection cuts are applied. Before these cuts are applied, the vertices produced by the vertex reconstruction algorithm are divided into the $1 \gamma 0 p$ and $1 \gamma 1 p$ topologies. The purpose of the pre-selection cuts is to reject reco-vertices with poorly reconstructed showers and/or tracks and to reduce the substantial cosmic background. At this stage multiple reco-vertices can be present in each event. Values for the pre-selection cuts were chosen for physicsbased reasons or because the the subsequent BDT background rejection stages would always choose to place those cuts. The following list of pre-selection cuts are applied to both the $1 \gamma 0 p$ and $1 \gamma 1 p$ topologies:

1. Fiducial cut: the distance between reco-vertices and each TPC wall $>10$ $\mathrm{cm}$ to improve containment of reco-vertices. Ensures uniformity of the drift electric field for the reco-vertices in the analysis while also reducing recovertices reconstructed from tracks and showers that enter from outside the TPC. Such tracks and showers can be produced by BNB neutrino interactions that occur in the cryostat but outside the TPC, or cosmic activity.

2. Shower energy: reconstructed shower energy $>30 \mathrm{MeV}$. This improves the quality of the reconstructed showers in the analysis and reduces the number of reco-vertices with associated Michel electron showers. Shower energy is determined using the method described in Chapter 4. Cut value based on BDT output.

3. Light in beam-gate: total photo-electrons (PE) detected by the PMTs inside the $3.2-4.8 \mu$ s beam-gate $>20$. BNB neutrino-argon interactions will typically produce a light in time with the in the beam-gate. Low total PE in the beam-gate indicates of a lack of ionizing particles produced in-time with the beam-spill, meaning either a neutrino from the spill produced only neutral non-photon particles or there were no neutrino interactions. Cut value based on BDT output.

The remaining pre-selection cuts are for the $1 \gamma 1 p$ topology only and utilize information from the associated track:

4. Track length maximum: associated track length $<100 \mathrm{~cm}$. A cut on long muon tracks which are not part of any signal topology in the analysis. This reduces background from $\nu_{\mu} \mathrm{CC}$ interactions and cosmics. Cut value based on studying signal and background distributions of the longest associated track length (see the left plot of Figure 5.23). 
5. Correct direction of track: ratio of the mean $\mathrm{dE} / \mathrm{dx}$ of the second half of the track to the first half must be $>1$. Introduced to remove tracks whose direction has been flipped by reconstruction. This flipping does not affect vertex reconstruction which treats both ends of a shower equally but variables such as track direction and angle will be incorrect. Cut value based on BDT output.

6. Back-to-back tracks and showers: the angle between the reconstructed track and shower must be $\cos \theta_{\gamma p}>-0.95$ and $\cos \theta_{\gamma p}<0.95$. This cut primarily targets tracks that have been erroneously split into two parts by reconstruction and one part has been mis-reconstructed as a shower. Cut value based on the fact that virtually all $1 \gamma 1 p$ vertices that did not pass this cut were incorrectly reconstructed split tracks.

7. Shower-vertex gap: distance between shower start and reco-vertex > $1 \mathrm{~cm}$ : Targets electron showers by requiring at least a small gap between the shower start and the reco-vertex. Photon showers will be mostly unaffected due to the $14 \mathrm{~cm}$ photon conversion length in liquid argon. This cut is not possible for the $1 \gamma 0 p$ topology as the vertex position is not known. Cut value chosen to ensure a small but visible gap between reco-vertex and shower start, increasing likelihood of selecting photon showers (see the right plot of Figure 5.23).
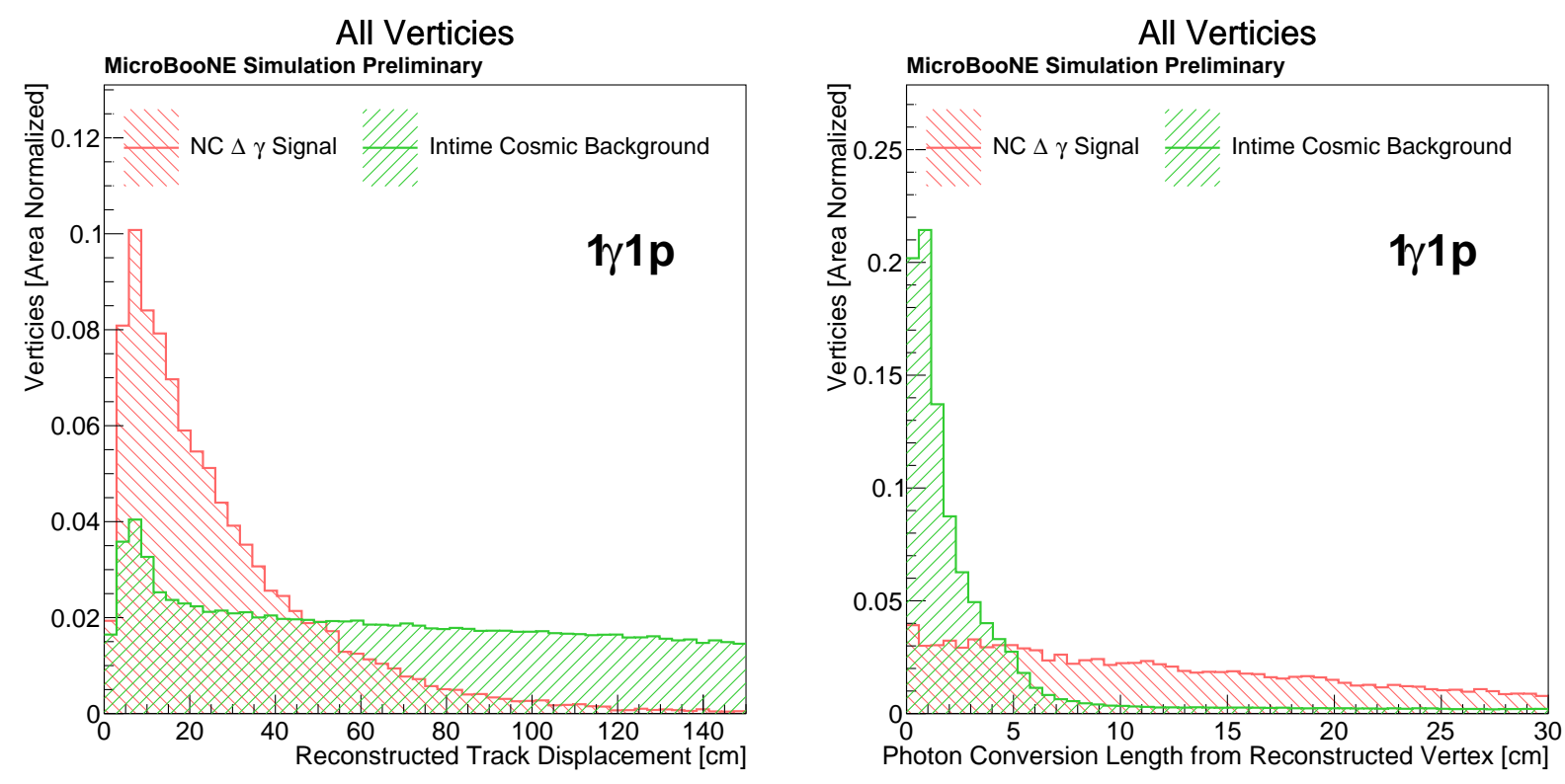

Figure 5.23: Distributions for the $1 \gamma 1 p$ NC $\Delta$ radiative signal and in-time cosmic background reco-vertices. Left: length of track associated with the vertex, right: gap between vertex and shower start. 


\subsection{Boosted Decision Tree Background Rejection}

After pre-selection the remaining reco-vertices are passed to the BDT background rejection stage. Prior to the use of BDTs as part of the analysis event selection, a simple series of one-dimensional cuts (similar to the pre-selection cuts described in the previous section) was used to isolate signal from background. This simple cut-based approach was optimized in terms of statistical significance $\left(\frac{s}{\sqrt{b}}\right)$ by optimizing each of the cuts in the order they were applied to the signal and background samples. This approach proved moderately effective at isolating signal and rejecting background, however given the rarity of $\mathrm{NC} \Delta$ radiative decay and the prevalence of background for the low energy photon search, the approach did not give strong enough signal-background separation to make it a viable choice for the analysis. BDTs are a far more powerful form of event classification than simple cut basedapproaches. While experience with BDTs in particle physics is limited, they tend to perform well in an event classification context compared to other event selection methods, such as machine learning, when little tuning has been applied. BDTs are also typically unaffected by the inclusion of variables that do not discriminate between signal and background [2] and have been shown to have potential for signal identification in the context of the MiniBooNE LEE [81]. For these reasons a BDTbased approach to event selection was chosen for the analysis.

This analysis uses the Toolkit for Multivariate Analysis (TMVA) [2] BDT implementation found in ROOT [82]. BDTs use multiple different decision trees to separate signal and background events. Decision trees can be used for event classification by giving them a set of variables associated with signal and background events. Decision trees in TMVA have a binary structure with each node in the tree being a cut on an event variable as shown in Figure 5.24. When a cut is placed on a set of events at a specific node, rather than reject the events that fail to pass the cut, the input event set is split into two and passed to two new decision nodes in the tree. This allows a decision tree to isolate multiple regions of event variable space as being either signal or background-like, as opposed to a traditional box-cut approach which can only isolate one region.

Single decision trees can be vulnerable to statistical fluctuations in their training samples. A statistical fluctuation in just one variable can cause a chain reaction of changes to cut variables and values further down the tree. A boosted decision tree counteracts this by constructing a "forest" of multiple decision trees and considers the output of all of them to identify an event as signal or background. Events used to train the decision tree forest undergo a process called "boosting" where the 


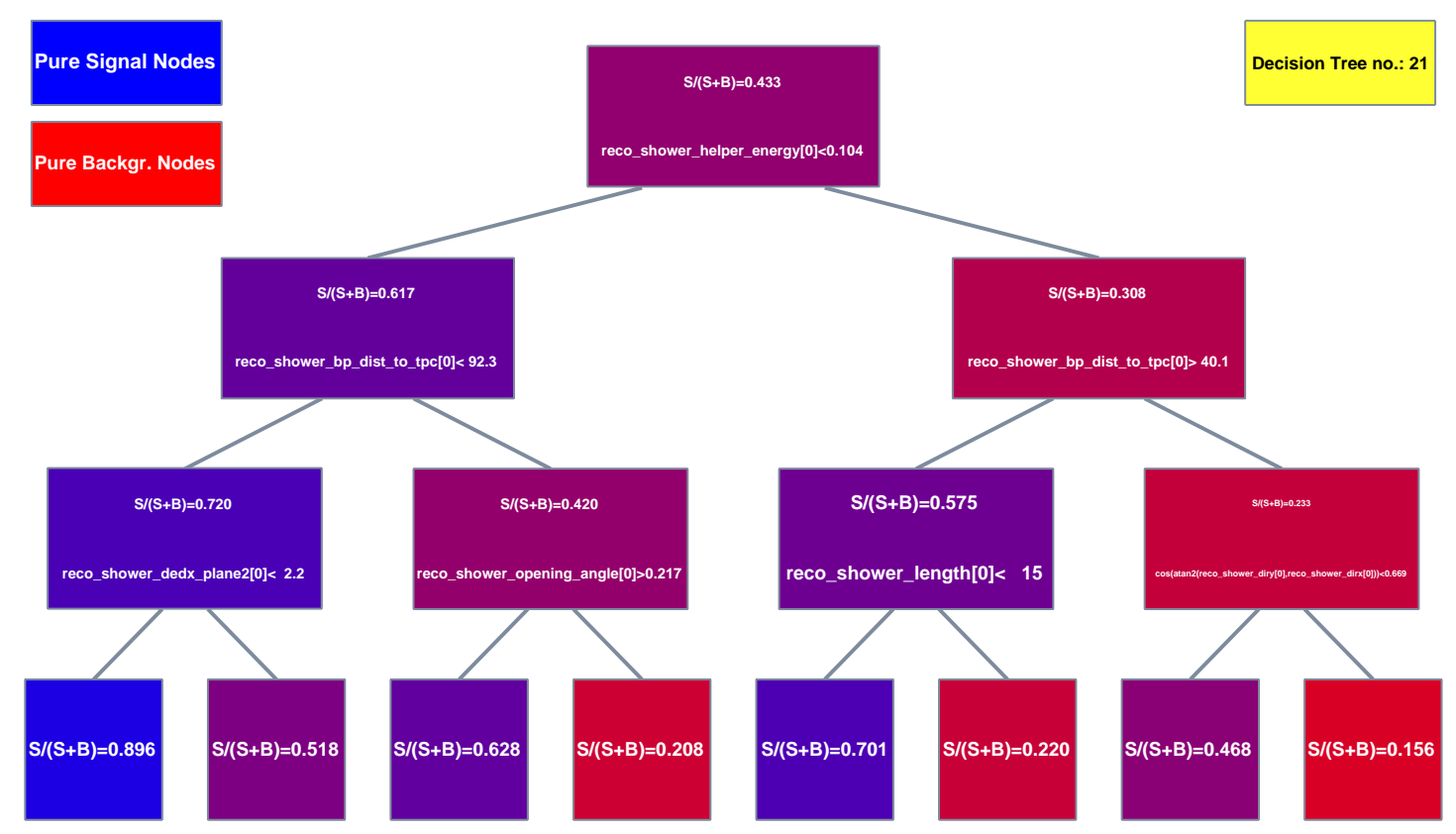

Figure 5.24: An example decision tree built using the $\mathrm{NC} \Delta$ radiative sample as signal and the in-time cosmic sample as background. Each node has a selection of signal (S) and background (B) events associated with it. Each node shows the signal purity of the associated events $(\mathrm{S} /(\mathrm{S}+\mathrm{B}))$ and the optimal cut variable and value for the node. The nodes are coloured based on signal purity: nodes with a greater fraction of signal events will tend towards a blue and nodes with a greater fraction of background events will tend towards a red. This example decision tree has a depth of 3 instead of the standard 4 used in the analysis to improve readability of the tree node cuts.

events will have a weight applied to them based on the results of the previous decision tree. Events that were mis-classified by the previous decision tree (background events classified as signal or signal events classified as background) will have a higher weight assigned to them focusing the tree being trained on events the previous tree had difficulty classifying.

To construct a decision tree, TMVA is supplied with a set of variables (such as shower energy and track length) and a set of input signal and background training event samples. The variable used for the cut and the cut value for the first node of the tree is chosen by determining the variable-value combination that maximizes the signal-background separation of the input events. The cut is placed, the events that pass the cut are sent to one new decision node and the events that fail are passed to another. The process for determining the variable-value cut combination for the first node is then repeated for the two new nodes using the corresponding (passed or failed) events received from the previous node. This process can be repeated until there is only one signal or background event in each leaf-node (the nodes at the end 
of the tree), however doing so would result in a decision tree that is highly overtrained on the training samples. To prevent overtraining in this manner, the number of times a pair of new nodes is created down the tree structure (tree-depth) is typically capped. Furthermore, in order to place a cut on the events passed to a given decision node, the ratio of events to the total number of input training events must be above a certain threshold. For this analysis the maximum tree-depth is four and the minimum number of events in a node in order to place a cut is $2.5 \%$ of the total.

Pruning is a process by which statistically insignificant nodes in a decision tree are removed to reduce the likelihood of overtraining the tree. For normal, deep decision trees this can be a valuable tool to improve reliability, however boosted decision trees use a typically use a forest of limited-depth decision trees for classification (a depth of four for this analysis). This limited depth makes pruning unnecessary as the manually dictated depth of the decision trees will be far stronger than the use of a pruning algorithm [2]. For this reason pruning was not performed on the BDTs used in this analysis.

Correlations between BDT variables can cause a loss in performance in the ability of the BDT to classify signal and background events. TMVA can apply a selection a transformations to the input training variables such as transforming the variables to a normalized Gaussian shape and a linear transformation into a non-correlated variable space. Attempting to de-correlate non-Gaussian distributed variables and variables with non-linear correlations will result in little performance gain and performance can be worsened if variables are highly non-linearly correlated. Both the Gaussian and linear de-correlation transformations are used in the analysis to decorrelate training variables and increase the classification performance for each BDT.

The BDT background rejection stage of event selection is divided into two main sections: cosmic background BDT rejection and BNB background BDT rejection. These two sections are further subdivided into the $1 \gamma 0 p$ and $1 \gamma 1 p$ topologies resulting in a total of four BDTs used in the analysis. The $1 \gamma 0 p$ topology cosmic and BNB BDTs are both trained on a set of shower related variables. The $1 \gamma 1 p$ topology cosmic and BNB BDTs have access to the same shower-related variables as the $1 \gamma 0 p$ topology as well as additional track-related variables.

\subsubsection{Training}

Some of the variables listed below are in terms of the following: $\vec{s}_{\text {start }}$ is the shower start position, $s_{\text {start }[\mathrm{x}, \mathrm{y}, \mathrm{z}]}$ denotes the $\mathrm{x}, \mathrm{y}$ and $\mathrm{z}$ components of the shower start, $\vec{s}_{\text {end }}$ 
is the shower end position, $\hat{s}_{\mathrm{dir}}$ is the shower direction unit vector, $s_{\operatorname{dir}[\mathrm{x}, \mathrm{y}, \mathrm{z}]}$ denotes the $\mathrm{x}, \mathrm{y}$ and $\mathrm{z}$ components of the shower direction unit vector, $\hat{t}_{\mathrm{dir}}$ is the track direction unit vector and $t_{\operatorname{dir}[\mathrm{x}, \mathrm{y}, \mathrm{z}]}$ denotes the $\mathrm{x}, \mathrm{y}$ and $\mathrm{z}$ components of the track direction unit vector. The reconstructed shower related variables used to train all four BDTs are:

1. Shower energy $(\mathrm{GeV})$ : Described in pre-selection. Calculated from the plane on which the shower has the largest number of associated hits.

2. Shower $\mathrm{dE} / \mathrm{dx}$ in the collection plane $(\mathrm{MeV} / \mathrm{cm})$ : Calculated using the method described in Chapter 4.

3. Shower length $(\mathbf{c m})$ : The length of the shower described in terms of a 3 -d cone.

4. Shower opening angle (radians): The opening angle of the shower described in terms of a 3 -d cone (see Section 4.3).

5. Backwards-projected distance from shower start to TPC wall $(\mathrm{cm})$ : The shower is backwards-projected and the point at which the backwardsprojection intersects with a TPC wall is found. This variable is the distance between this intersection and the shower start.

6. Distance from shower to closest flash in $z(\mathbf{c m})$ : Shortest z-axis distance between an in-beam-gate reconstructed flash and the z-axis projection of the shower associated with the reco-vertex. The shower z-axis projection is defined as a range from $s_{\text {startz }}$ to $s_{\text {startz }}+s_{\text {length }} \cdot s_{\text {dirz }}$. Value is set to zero if the flash resides within the closest shower projection.

7. Shower $\operatorname{cosine} \boldsymbol{\theta}_{\boldsymbol{y z}}$ : Cosine of the angle of the shower direction on the $\mathrm{y}-\mathrm{z}$ plane. $\theta_{y z}=\arctan \left(s_{\text {diry }} / s_{\text {dirz }}\right)$.

8. Shower $\operatorname{cosine} \boldsymbol{\theta}_{y x}$ : Cosine of the angle of the shower direction on the $\mathrm{y}-\mathrm{x}$ plane. $\theta_{y x}=\arctan \left(s_{\text {diry }} / s_{\text {dirx }}\right)$.

9. Shower end point in $\boldsymbol{x}, \boldsymbol{y}, \boldsymbol{z}(\mathbf{c m})$ : Estimated using shower start point, direction and length. $\vec{s}_{\text {end }}=\vec{s}_{\text {start }}+s_{\text {length }} \cdot \hat{s}_{\text {dir }}$.

10. Reco-vertex $\boldsymbol{x}, \boldsymbol{y}, \boldsymbol{z}$ position (cm): Reco vertex position determined by the vertex reconstruction stage of selection described in Section 5.1 .

\section{Distance from reco-vertex to nearest TPC wall $(\mathrm{cm})$.}


12. Number of showers with start points within $10 \mathrm{~cm}$ of the vertex: The primary focus of this variable is $\pi^{0} \rightarrow 2 \gamma$ decay from BNB interactions where only one photon shower was associated with the reco-vertex.

In absence of a known neutrino reco-vertex position, the $1 \gamma 0 p$ sample uses the start of the shower for reco-vertex $x, y, z$ position and to calculate the distance from recovertex to nearest the TPC wall. The track related variables used to train the cosmic and BNB background rejection BDTs for the $1 \gamma 1 p$ topology only are:

13. Track energy (GeV). Calculated using calorimetric information.

14. Track length $(\mathbf{c m})$ : Distance between the start and end of the track.

15. Shower-start-reco-vertex gap $(\mathbf{c m})$ : Distance between shower start and reco-vertex. The primary focus of this variable is separating photon and electron showers.

16. Invariant mass squared of track-shower pair assuming a photon shower and proton track $\left(\mathbf{G e V}^{\mathbf{2}}\right): W_{\Delta}^{2}=m_{p}^{2}+2\left(E_{p} E_{\gamma}-\left|p_{p}\right|\left|p_{\gamma}\right| \cos \theta_{p \gamma}\right)$, where $W_{\Delta}$ is the reconstructed invariant mass, $m_{p}$ is the mass of a proton, $E_{p}$ is the total energy of the "proton" track (track energy $+m_{p}$ ), $E_{\gamma}$ is the energy of the "photon" (shower energy), $\left|p_{p}\right|$ is the magnitude of momentum of the "proton" track $\left(\sqrt{E_{p}^{2}-m_{p}^{2}}\right),\left|p_{\gamma}\right|$ is the magnitude of momentum of the "photon" (shower energy) and $\theta_{p \gamma}$ is the track-shower opening angle (see below).

17. Cosine of the track-shower opening angle: $\cos \theta_{t s}=\hat{t}_{\mathrm{dir}} \cdot \hat{s}_{\mathrm{dir}}$

18. Track mean $\mathrm{dE} / \mathrm{dx}(\mathrm{MeV} / \mathrm{cm})$ : Mean $\mathrm{dE} / \mathrm{dx}$ over the whole length of the track.

19. Ratio of the mean $\mathrm{dE} / \mathrm{dx}$ of the second half of the track to the first half: A measure of track direction flipped by reconstruction.

20. Track proton PIDA (particle identification A): PIDA of a proton candidate is given by the average of the $A$ values of all hits in a track track assuming a proton Bragg peak formula, $\langle A\rangle=(\mathrm{dE} / \mathrm{dx}) R^{-0.42}$, where $R$ is the residual range of the track [83].

21. Track Bragg-fit parameter $\boldsymbol{A}$ : a fit of $A$ is performed on the Bragg-peak formula with the $\mathrm{dE} / \mathrm{dx}$ and residual range of the track.

22. Track $\operatorname{cosine} \boldsymbol{\theta}_{\boldsymbol{y} \boldsymbol{z}}$ : Cosine of the angle of the track direction on the Y-Z plane. $\theta_{y z}=\arctan \left(t_{\text {diry }} / t_{\text {dirz }}\right)$. 
23. Track $\operatorname{cosine} \boldsymbol{\theta}_{\boldsymbol{y} \boldsymbol{x}}$ : Cosine of the angle of the track direction on the Y-X plane. $\theta_{y x}=\arctan \left(t_{\text {diry }} / t_{\text {dirx }}\right)$.

\section{Track end point in $\boldsymbol{x}, \boldsymbol{y}, \boldsymbol{z}(\mathrm{cm})$.}

This list of training variables was arrived at after multiple previous training variable list iterations. The first iteration of the list contained a smaller number of variables, chosen for physics based reasons primarily focused on cosmic rejection, electron shower rejection and muon track rejection. These include shower energy: chosen primarily to differentiate between $\mathrm{NC} \Delta$ showers and low-energy cosmic showers, shower $\mathrm{dE} / \mathrm{dx}$ : to differentiate between photon and electron showers, distance from shower to closest flash in $z$ : to reject cosmic showers out of time with the beam, shower cosine $\theta_{y z}$ : to reject downward facing cosmic showers, reco vertex $x, y, z$ : to reject cosmic vertices closer to the edges of the TPC, track length: to reject long muon tracks both cosmic and from $\nu_{\mu}$ CC interactions, shower-start-reco-vertex distance: to reject electron showers with no gap between the reco-vertex and shower start and track cosine $\theta_{y z}$ : to reject downward facing cosmic tracks. From this base the remaining variables were gradually added in trial-and-error by studying signal and background distributions of potentially impactful variables and the effect of adding those variables on BDT performance. Variables considered but not used in the final BDT variable list include shower $\mathrm{dE} / \mathrm{dx}$ values from all planes (as opposed to just the collection plane), the total PE sum before the beam-gate: an attempt to reject cosmics that produced light before the beam-spill arrived, the number of showers with start points within 20 and $30 \mathrm{~cm}$ of the vertex: less effective variations on a variable that is used and the number of tracks with start points within 10, 20 and $30 \mathrm{~cm}$ of the vertex: an attempt to correct for instances were a track-shower association was missed by the vertex reconstruction algorithm.

\subsubsection{Cosmic Rejection Boosted Decision Tree}

The cosmic rejection BDTs for the $1 \gamma 0 p$ and $1 \gamma 1 p$ topologies are trained using the $\mathrm{NC} \Delta$ radiative sample as signal and the in-time cosmic sample as background. Figures 5.25 to 5.29 show the shower and track related variable distributions used to train the $1 \gamma 1 p$ cosmic rejection BDT. Figures 5.30 and 5.31 show the BDT response of the $1 \gamma 0 p$ and $1 \gamma 1 p$ cosmic rejection BDTs and the efficiency of placing a cut on the response for the signal and background samples. The $1 \gamma 1 p$ response shows a far greater level of separation between signal and background than the $1 \gamma 0 p$ response highlighting, the impact of the additional track information available in the $1 \gamma 1 p$ topology. 
When training a BDT it can be informative to study the importance of each training variable on the BDT separation between signal and background. The TMVA implementation of BDTs determines variable importance based on the number of times a training variable is used to place the optimal cut in the node of a decision tree belonging to the BDT forest, weighted by the separation gained by placing the cut and the number of events in the node. Figure 5.32 shows the variable importance ranking for the $1 \gamma 0 p$ cosmic BDT. Shower energy is ranked as one of the most important variables for this BDT, as showers produced from cosmic activity are significantly lower in energy than photon showers produced by $\mathrm{NC} \Delta$ radiative decay (see the top left plot of Figure 5.25).

Distance from shower to closest in-beam-gate flash on the z-axis is another important variable for the $1 \gamma 0 p$ cosmic BDT, as it is a powerful discriminator between cosmic showers and BNB produced showers. BNB showers will almost always produce scintillation light in the beam-gate, as they are produced by the BNB neutrinos in the beam-spill. This light will be reconstructed into a flash as described in Section 4.4. As the closest in-beam-gate flash to the BNB shower will be produced by the shower itself, the distance between a BNB shower and the closest in-beam-gate flash will be small (see the bottom right plot of Figure 5.25). Cosmic showers will also produce light (albeit in smaller amounts due to the lower energy of cosmic showers), however the majority of cosmic showers will not arrive and produce light in the TPC during the beam-gate. Therefore the closest in-beam-gate flash to a cosmic shower will typically be a flash produced by BNB activity some distance away from the cosmic shower.

Figure 5.33 show the variable importance ranking for the $1 \gamma 1 p$ cosmic BDT. Track cosine $\theta_{y z}$ the most important variable for the $1 \gamma 1 p$ cosmic BDT as it is a powerful discriminator between cosmic tracks and BNB produced tracks. BNB produced tracks will tend to be aligned with the BNB beam-direction, while cosmic tracks will be downward facing as they are produced in the atmosphere and will enter the TPC from above. The shower-vertex gap is another strong variable for the $1 \gamma 1 p$ cosmic BDT. Showers produced by NC $\Delta$ radiative decay are photon showers and therefore have a $14 \mathrm{~cm}$ photon conversion length in liquid argon. Barring mis-reconstruction, all cosmic showers in the $1 \gamma 1 p$ will be Michel electron showers associated with cosmic muon tracks. As such there will be no gap between the shower-start and reco-vertex resulting in strong separation between the NC $\Delta$ radiative and in-time cosmic vertices for this variable.

Track mean $\mathrm{dE} / \mathrm{dx}$ and track mean $\mathrm{dE} / \mathrm{dx}$ ratio are particularly powerful vari- 
ables for the $1 \gamma 1 p$ cosmic BDT as they allow for the differentiation between stopping particles such as protons from NC $\Delta$ radiative decay and MIP tracks such as cosmic muons. Track mean $\mathrm{dE} / \mathrm{dx}$ will be approximately $2 \mathrm{MeV} / \mathrm{cm}$ for cosmic muon tracks and much higher for protons, which have a high rate of energy deposition as they come to a stop in the TPC. Similarly the mean $\mathrm{dE} / \mathrm{dx}$ ratio for a muon will be close to one as MIP $\mathrm{dE} / \mathrm{dx}$ is relatively constant. Protons are stopping particles and so have a changing rate of energy deposition as they travel, shifting the mean $\mathrm{dE} / \mathrm{dx}$ (end/start) ratio of the track they produce to values greater than one. 

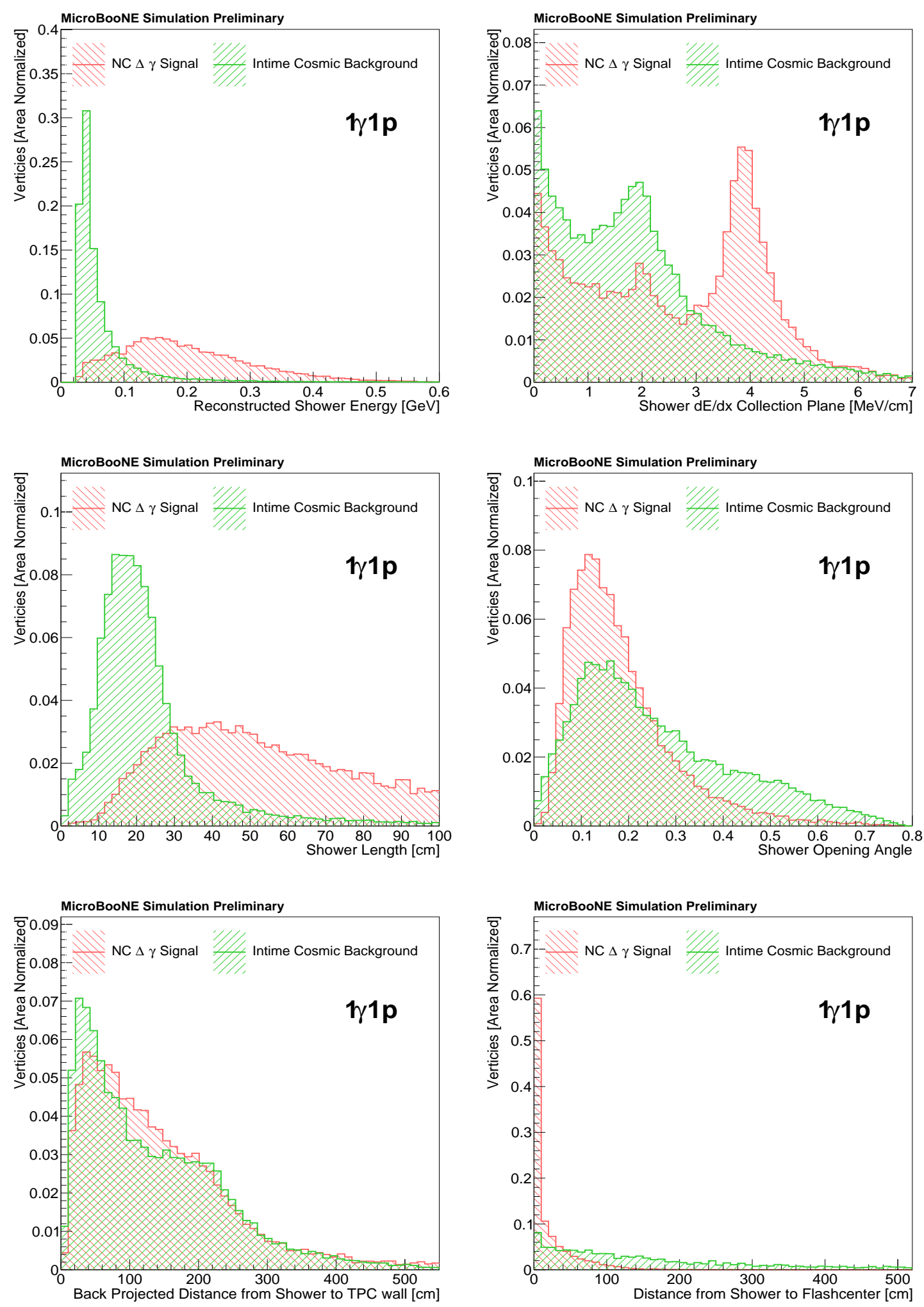

Figure 5.25: Training variables for the NC $\Delta$ radiative sample (red) and in-time cosmic sample (green) used to train the $1 \gamma 1 p$ cosmic rejection BDT. From top left to bottom right: shower energy, shower $\mathrm{dE} / \mathrm{dx}$, shower length, shower opening angle, backwards-projected shower distance from TPC wall and distance between shower and closest flash ( $z$-axis). 

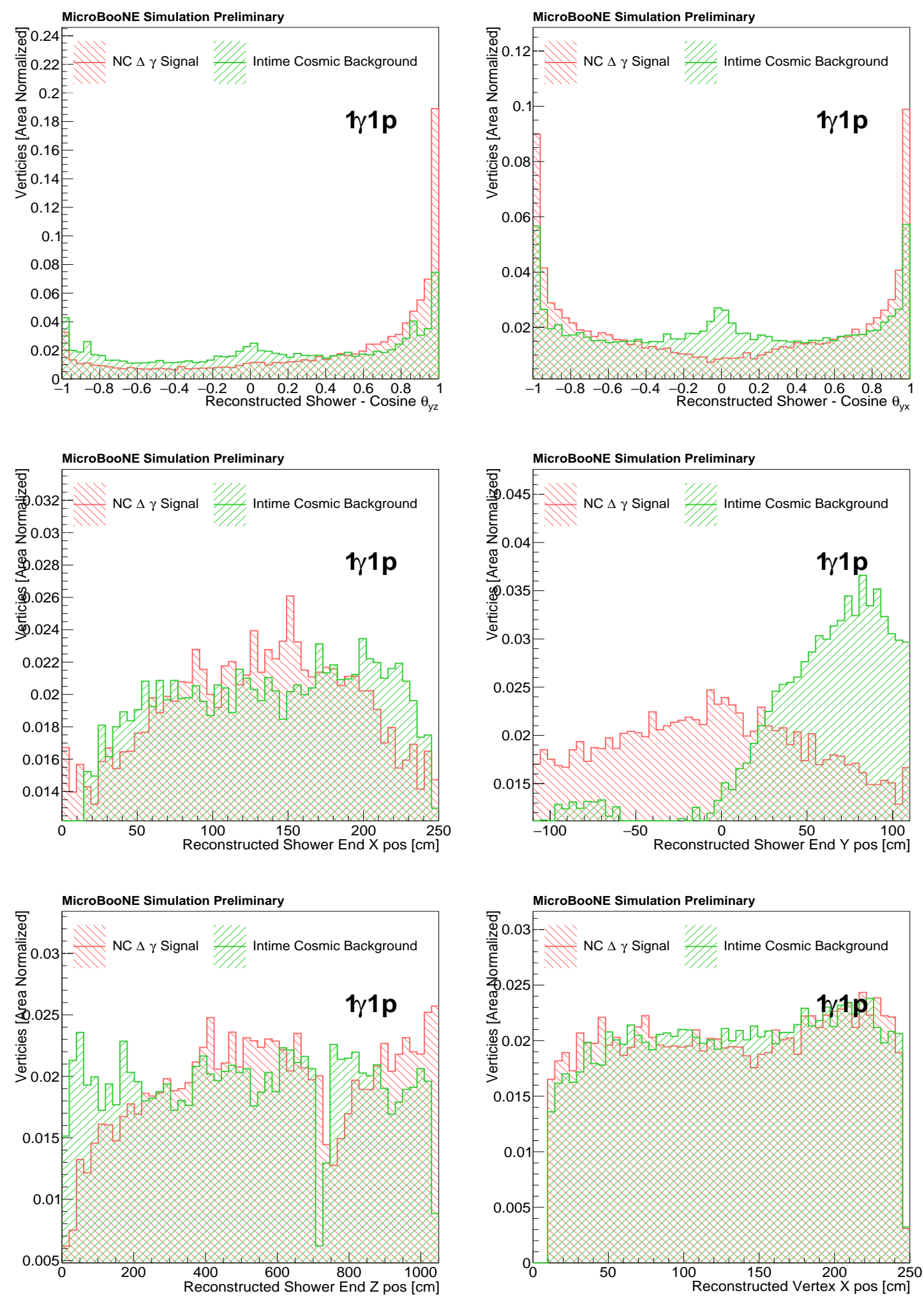

Figure 5.26: Training variables for the NC $\Delta$ radiative sample (red) and in-time cosmic sample (green) used to train the $1 \gamma 1 p$ cosmic rejection BDT. From top left to bottom right: shower $\cos \theta_{y z}$, shower $\cos \theta_{y x}$, shower end $x$-position, shower end $y$-position, shower end $z$-position and vertex $x$-position. 

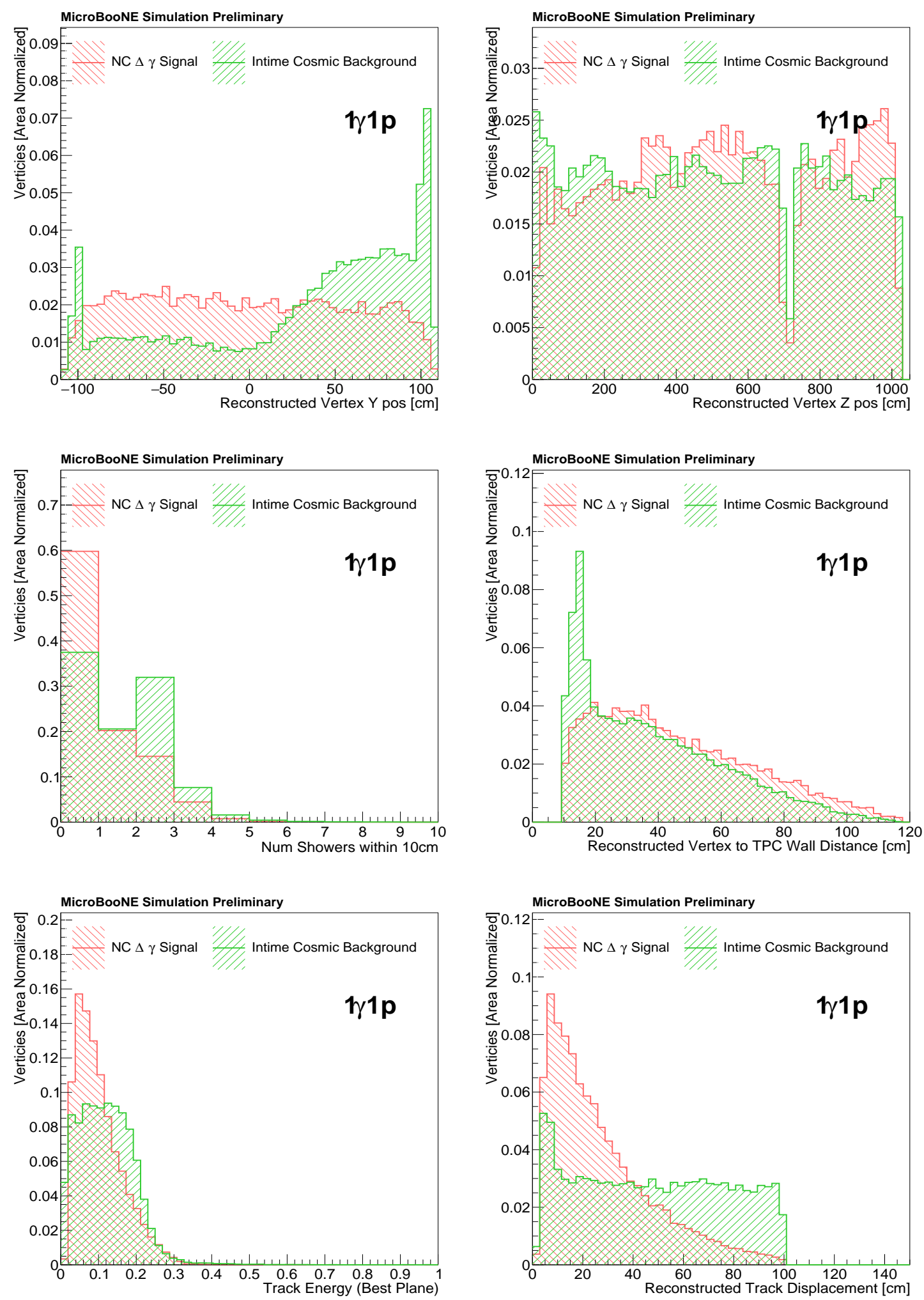

Figure 5.27: Training variables for the NC $\Delta$ radiative sample (red) and in-time cosmic sample (green) used to train the $1 \gamma 1 p$ cosmic rejection BDT. From top left to bottom right: vertex $y$-position, vertex $z$-position, number of showers within 10 $\mathrm{cm}$ of vertex, vertex-closest TPC wall distance, track energy and track length. 

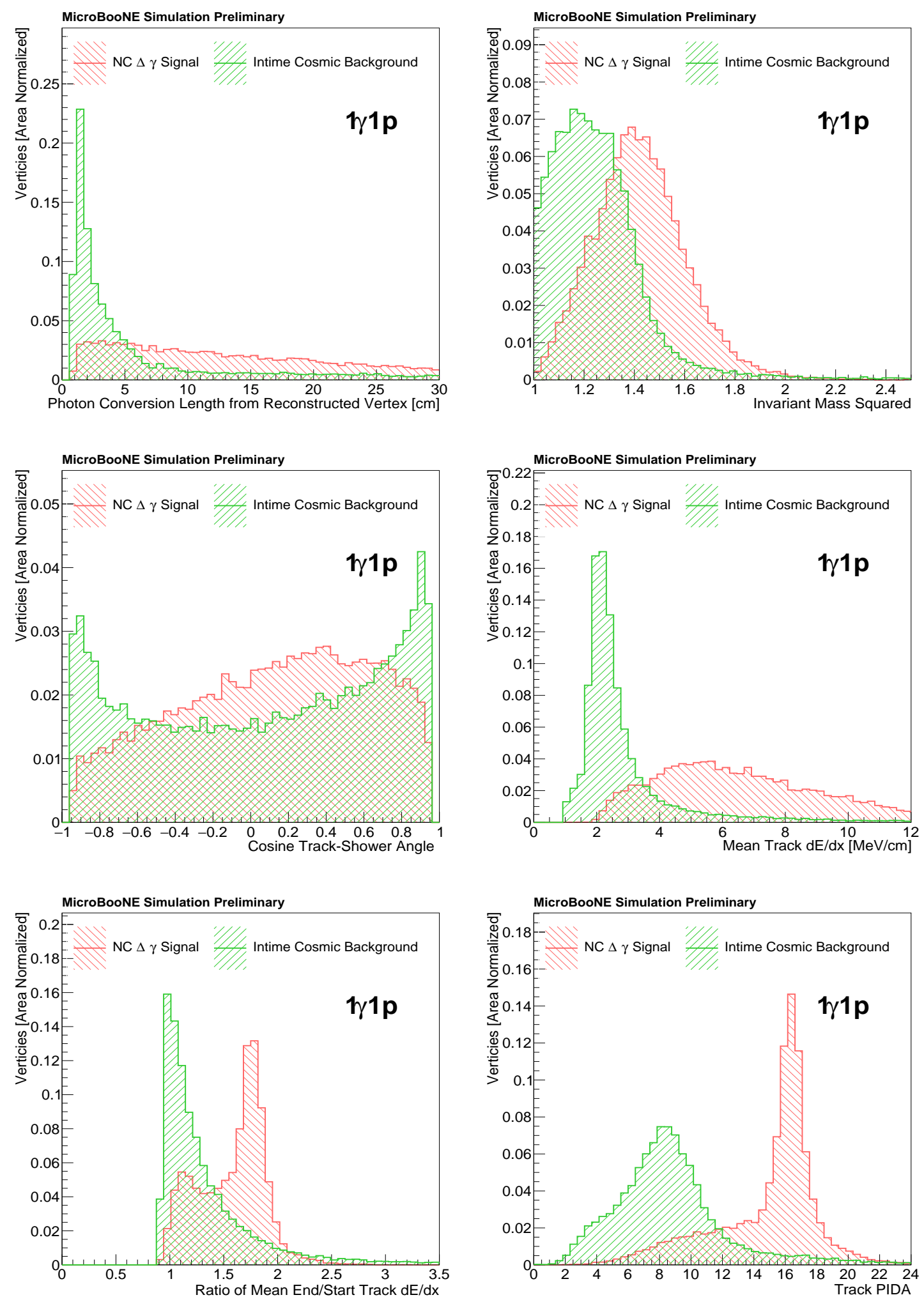

Figure 5.28: Training variables for the NC $\Delta$ radiative sample (red) and in-time cosmic sample (green) used to train the $1 \gamma 1 p$ cosmic rejection BDT. From top left to bottom right: shower start-vertex distance, track-shower invariant mass squared, cosine track-shower angle, mean track $\mathrm{dE} / \mathrm{dx}$, ratio of end-start mean track $\mathrm{dE} / \mathrm{dx}$ and track PIDA. 

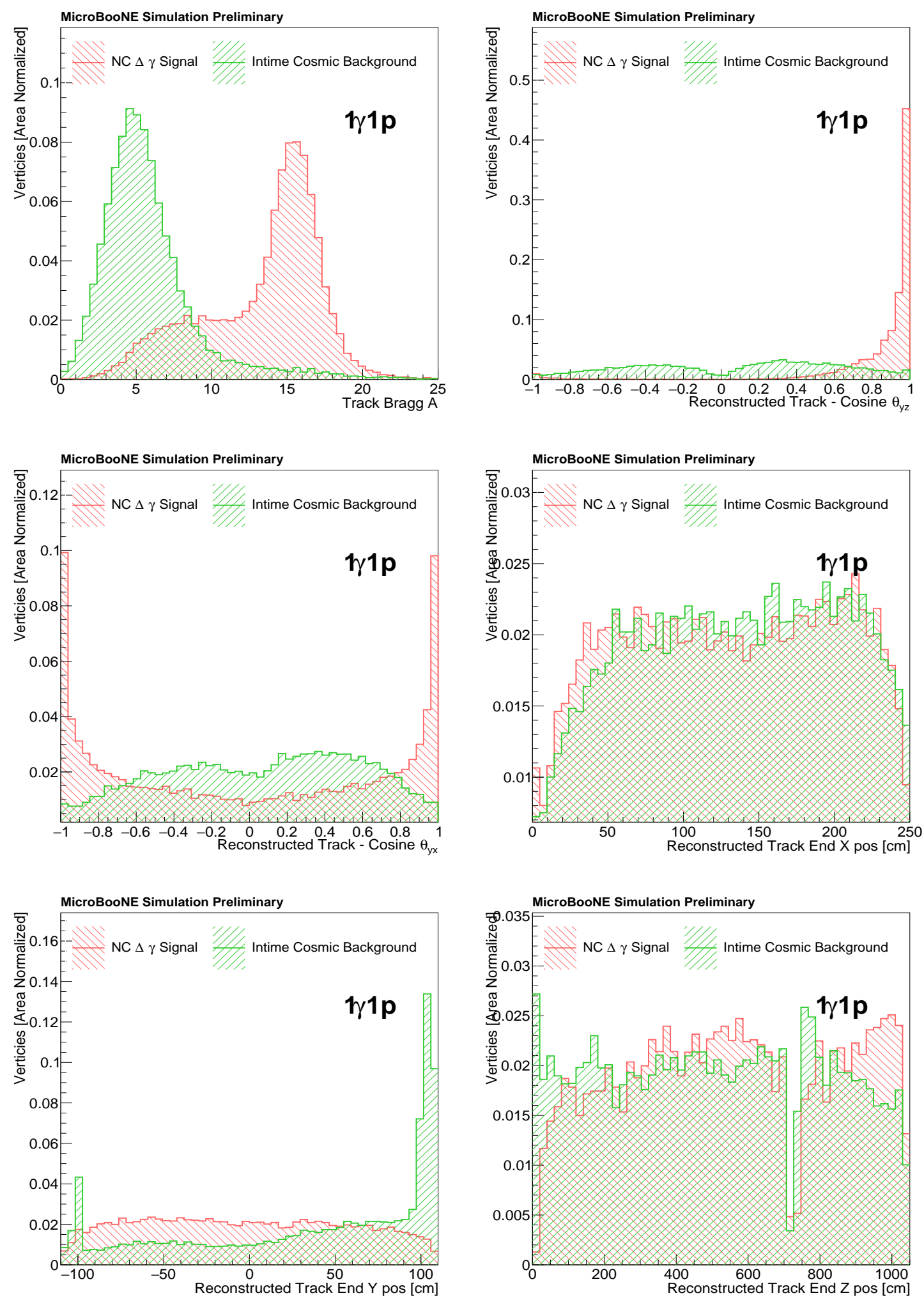

Figure 5.29: Training variables for the NC $\Delta$ radiative sample (red) and in-time cosmic sample (green) used to train the $1 \gamma 1 p$ cosmic rejection BDT. From top left to bottom right: track Bragg A value, track $\cos \theta_{y z}$, track $\cos \theta_{y x}$, track end $x$-position, track end $y$-position and track end $z$-position. 


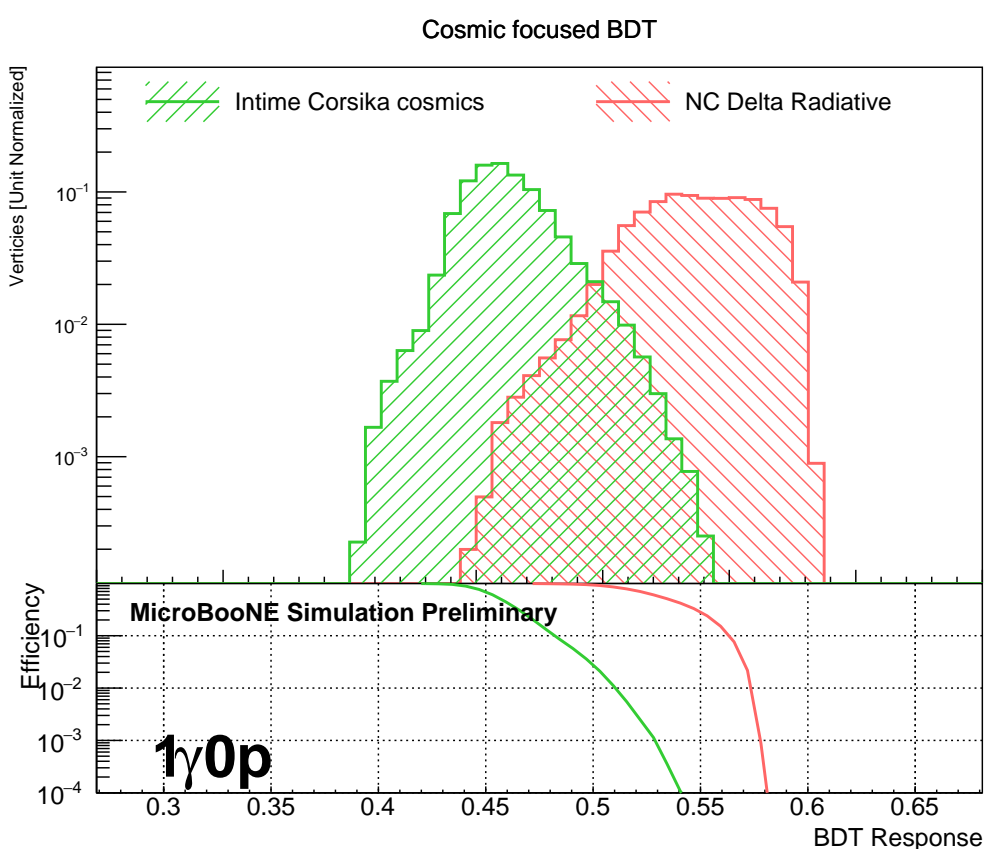

Figure 5.30: Cosmic rejection BDT response for the $1 \gamma 0 p$ topology. The top panel shows the area-normalized responses of the BDT to the NC $\Delta$ radiative reco-vertices (red) and in-time cosmic reco-vertices (green). The more signal-like a reco-vertex is considered to be the higher the response value will be. This is can be seen in the background and signal response distributions which are shifted to the left and right respectively. The bottom panel shows the effect of placing a BDT response cut on the efficiency of each sample.

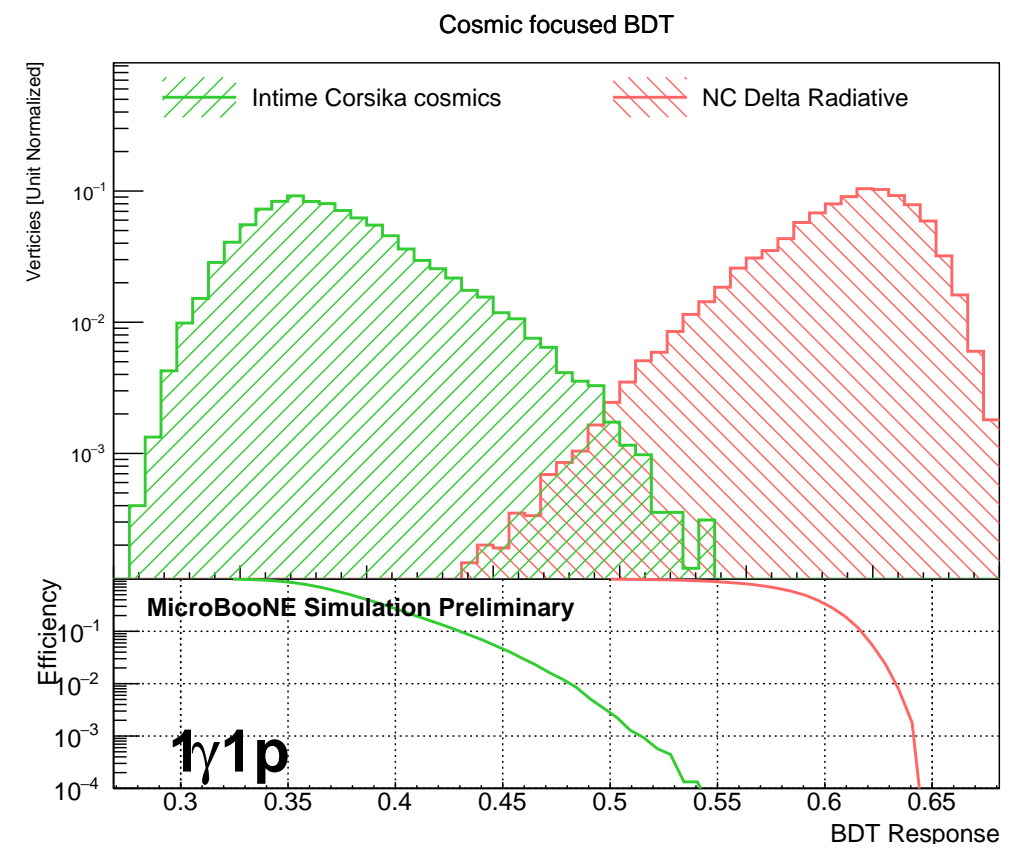

Figure 5.31: Cosmic rejection BDT response for the $1 \gamma 1 p$ topology. Displayed in the same manner as Figure 5.30. 


\section{1y0p Cosmic BDT}

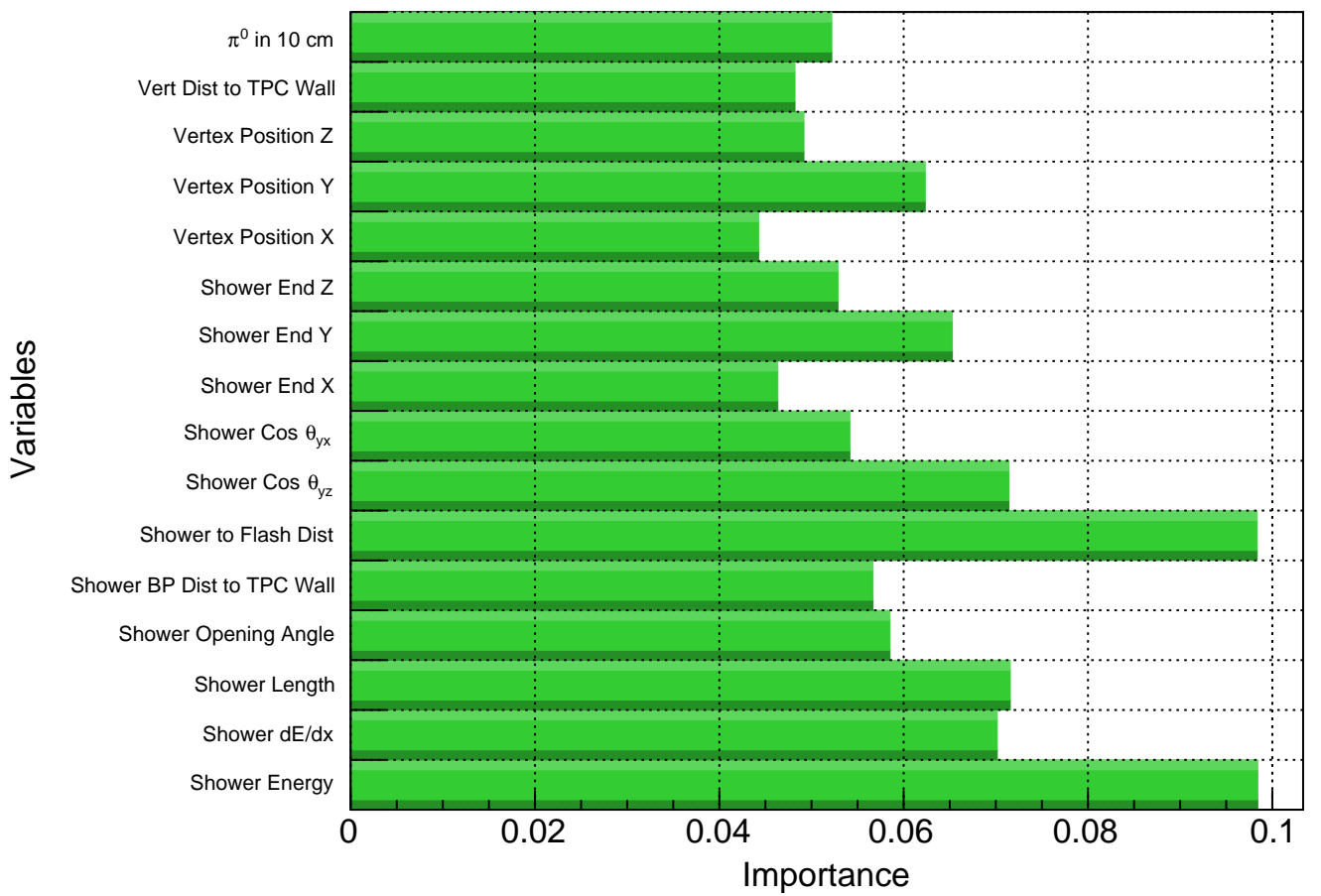

Figure 5.32: Variable importance ranking for the $1 \gamma 0 p$ cosmic rejection BDT.

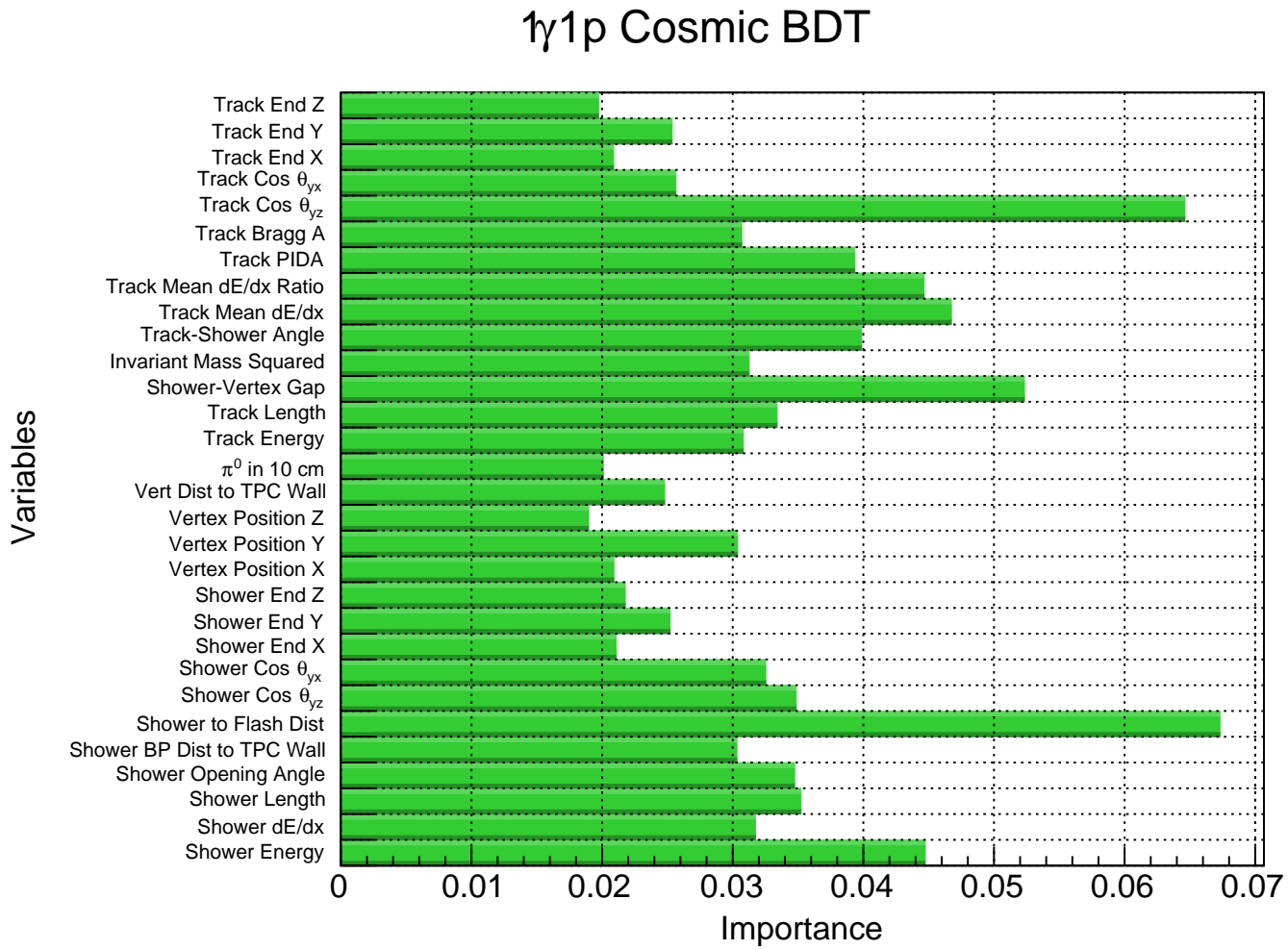

Figure 5.33: Variable importance ranking for the $1 \gamma 1 p$ cosmic rejection BDT. 


\subsubsection{BNB Rejection Boosted Decision Tree}

The BNB rejection BDTs for the $1 \gamma 0 p$ and $1 \gamma 1 p$ topologies are trained using the NC $\Delta$ radiative sample as signal and the BNB sample as background. Figures 5.34 to 5.38 show the shower and track related variable distributions used to train the $1 \gamma 1 p$ BNB rejection BDT. Figures 5.39 and 5.40 show the BDT response of the $1 \gamma 0 p$ and $1 \gamma 1 p$ BNB rejection BDTs and the efficiency of placing a cut on the response for the signal and background samples. As with the cosmic rejection BDT, the $1 \gamma 1 p$ topology performs better than the $1 \gamma 0 p$ topology.

Figure 5.41 shows the variable importance ranking for the $1 \gamma 0 p$ BNB rejection BDT. Shower energy is the most important variable for the BNB BDT as a large number of showers produced by BNB interactions are Michel electrons from $\nu_{\mu}$ CCQE interactions, much lower in energy than NC $\Delta$ radiative signal showers. Photon showers produced by $\pi^{0} \rightarrow 2 \gamma$ decay are also prevalent in background and in the case of a symmetric decay will be less energetic than signal showers (though in the case of an asymmetric $\pi^{0} \rightarrow 2 \gamma$ decay, these showers can look very similar to signal). Distance from shower to closest in-beam-gate flash on the $\mathrm{z}$-axis is a much less important variable for the BNB BDT compared to the cosmic BDT as, unlike the cosmic showers, background showers produced by the BNB will almost always produce light in the beam-gate.

Figure 5.42 shows the variable importance ranking for the $1 \gamma 1 p$ BNB rejection BDT. As for the $1 \gamma 1 p$ cosmic BDT, track cosine $\theta_{y z}$ is ranked as a very important variable for the $1 \gamma 1 p$ BNB BDT. As the $1 \gamma 1 p$ signal definition requires a $\Delta \rightarrow p+\gamma$ decay with only the photon and proton from that decay exiting the nucleus in truth, the tracks associated with signal vertices will tend to be forward-facing. The $1 \gamma 1 p$ BNB background vertices have contributions from any neutrino interaction simulated by GENIE with no constraint on the number of particles exiting the interaction nucleus. Many of these vertices will have multiple track producing particles in truth which are not properly reconstructed (the tracks are missed by reconstruction or merged into a single track). As a result the associated track of a BNB reco-vertex will not be as forward facing as the signal tracks on average. As a large number of tracks in the BNB background are muon tracks produced by $\nu_{\mu}$ CCQE interactions or charged pions, track mean $\mathrm{dE} / \mathrm{dx}$ and track mean $\mathrm{dE} / \mathrm{dx}$ ratio are powerful variables. This is for the same reason as for the $1 \gamma 1 p$ cosmic BDT: these variables are good at differentiating between stopping particles such as protons and MIPs such as muons. The shower-vertex gap is an important variable for the same reason as it was for the cosmic BDT: electron-photon differentiation via the expected $14 \mathrm{~cm}$ 
photon gap. 
Pre-Selection Cuts

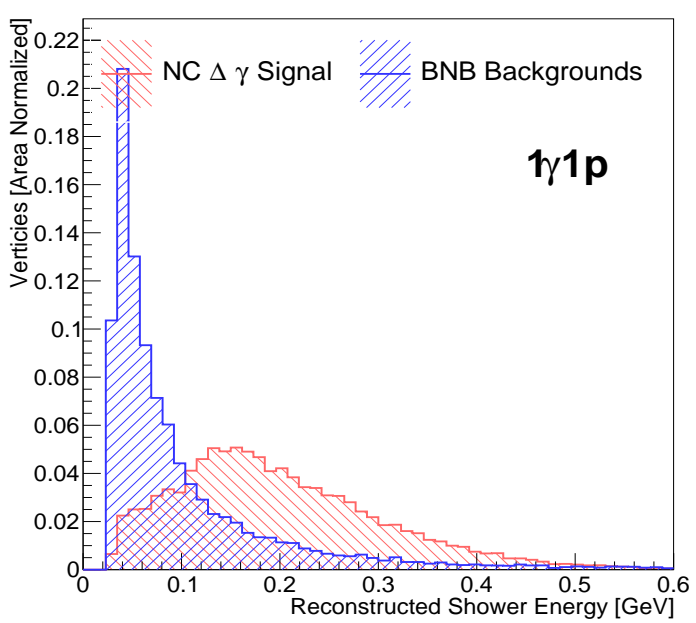

Pre-Selection Cuts

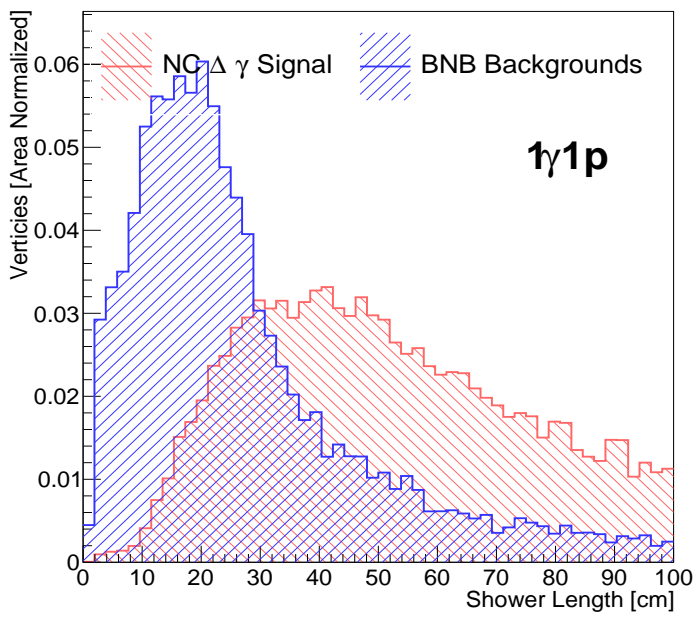

Pre-Selection Cuts

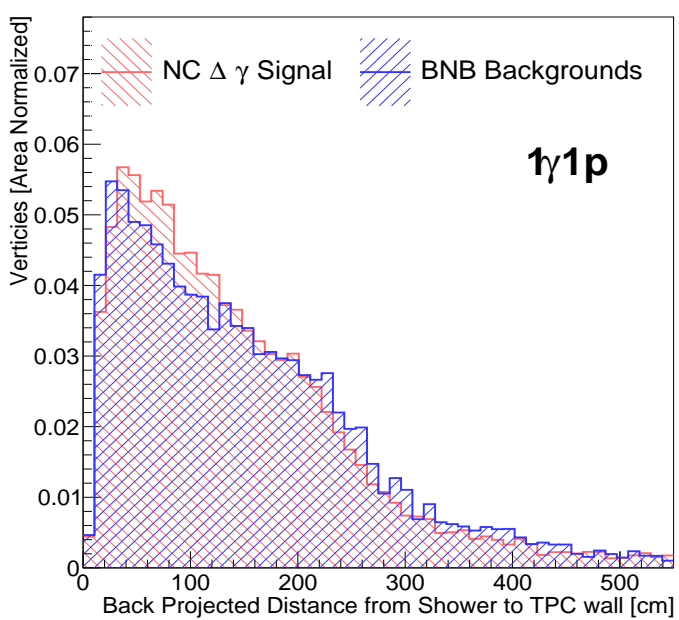

Pre-Selection Cuts

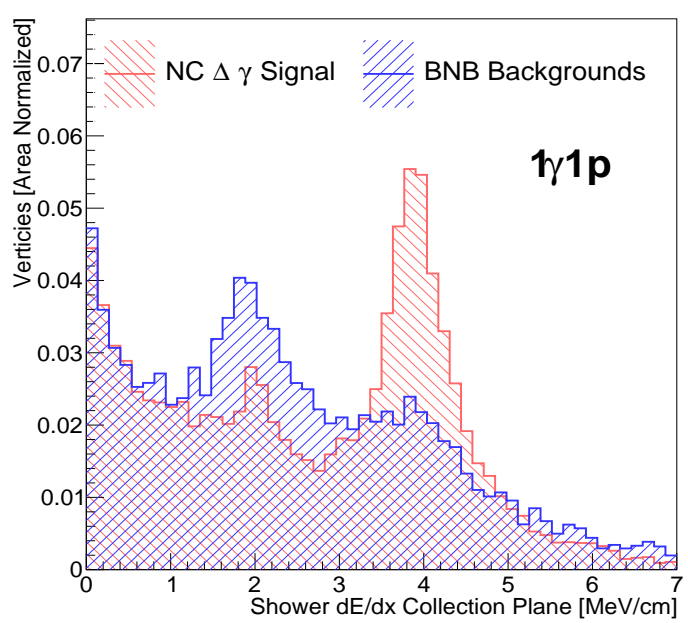

Pre-Selection Cuts

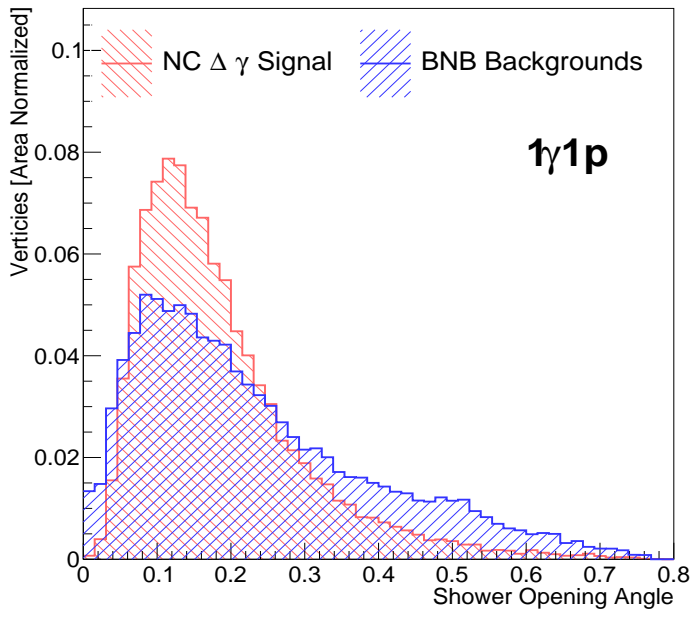

Pre-Selection Cuts

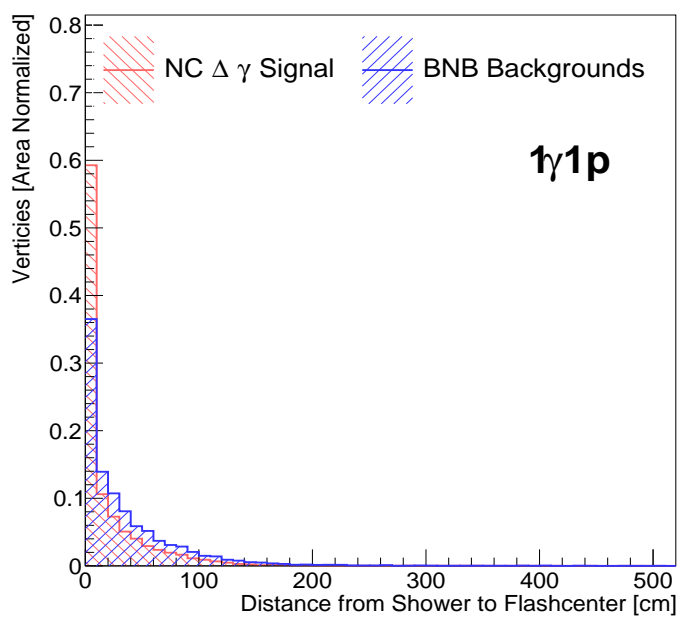

Figure 5.34: Training variables for the NC $\Delta$ radiative sample (red) and BNB sample (blue) used to train the $1 \gamma 1 p$ BNB rejection BDT. From top left to bottom right: shower energy, shower $\mathrm{dE} / \mathrm{dx}$, shower length, shower opening angle, backwardsprojected shower distance from TPC wall and distance between shower and closest flash ( $z$-axis). 
Pre-Selection Cuts

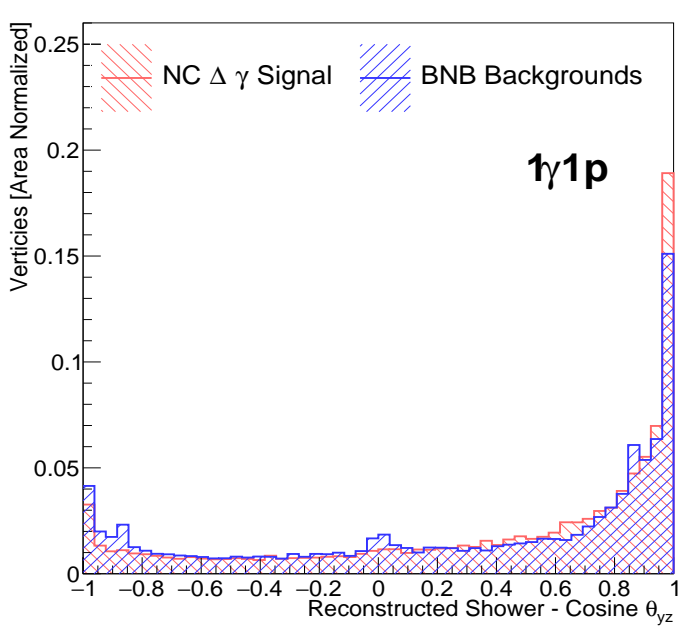

Pre-Selection Cuts

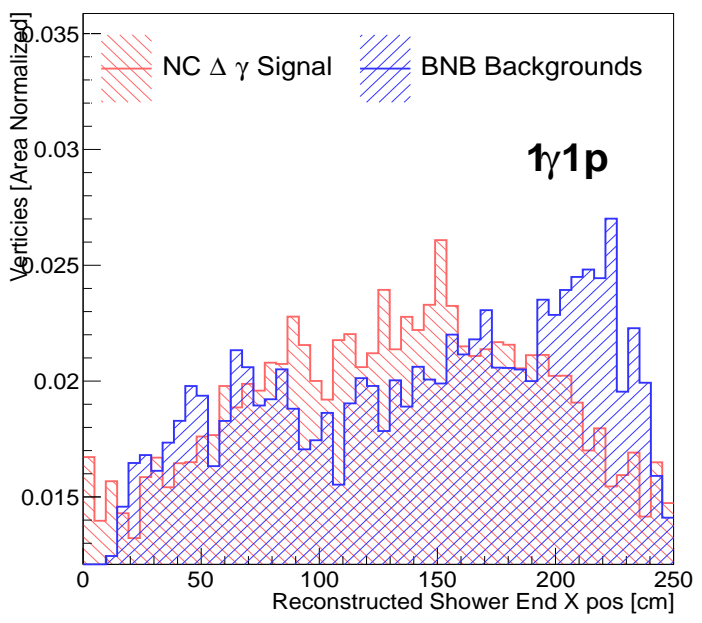

Pre-Selection Cuts

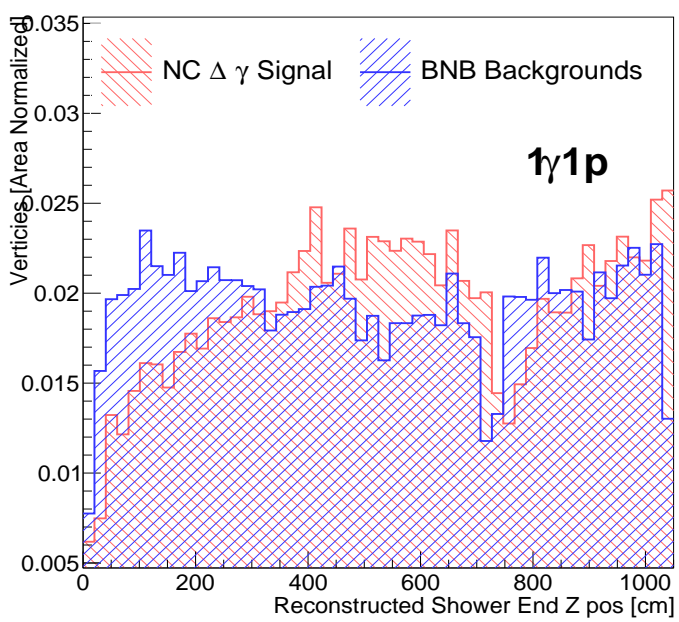

Pre-Selection Cuts

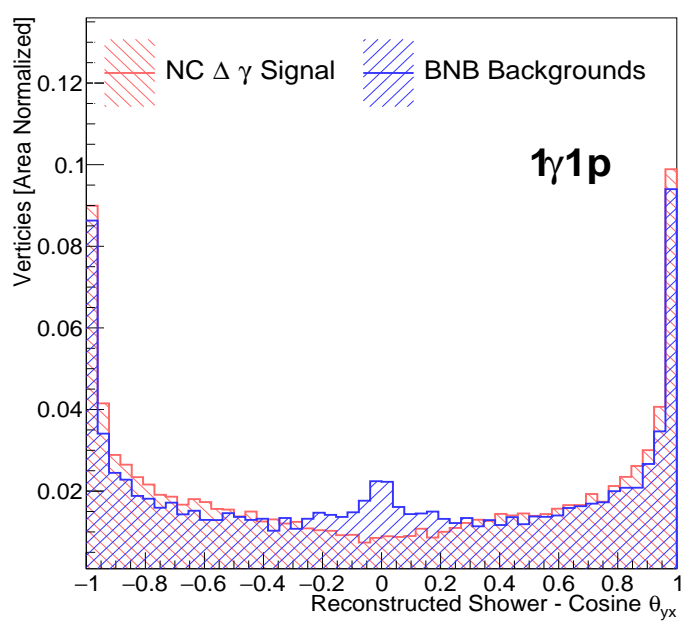

Pre-Selection Cuts

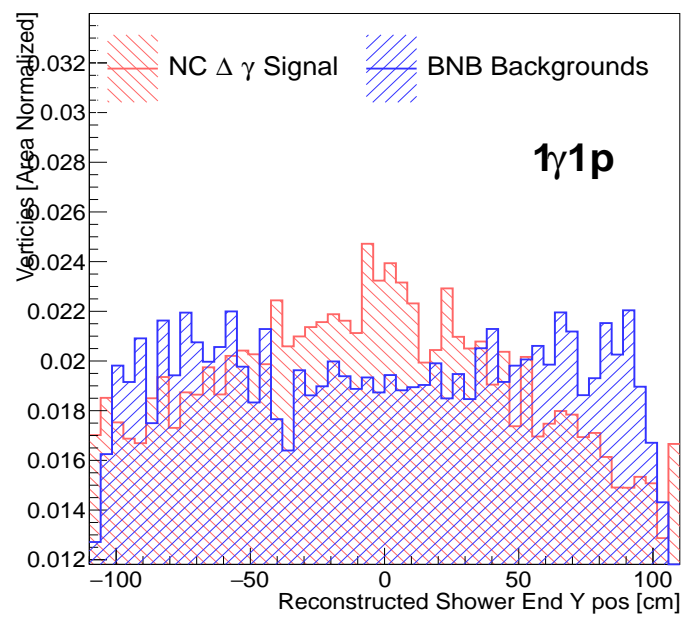

Pre-Selection Cuts

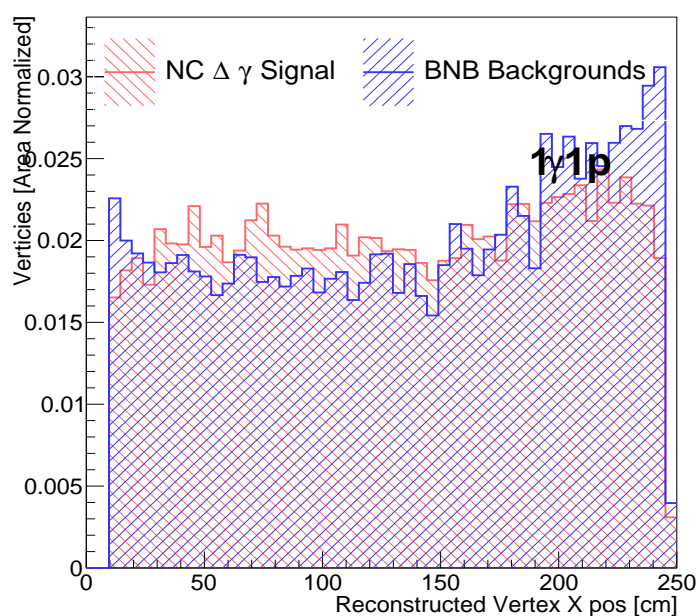

Figure 5.35: Training variables for the NC $\Delta$ radiative sample (red) and BNB sample (blue) used to train the $1 \gamma 1 p$ BNB rejection BDT. From top left to bottom right: shower $\cos \theta_{y z}$, shower $\cos \theta_{y x}$, shower end $x$-position, shower end $y$-position, shower end $z$-position and vertex $x$-position. 
Pre-Selection Cuts

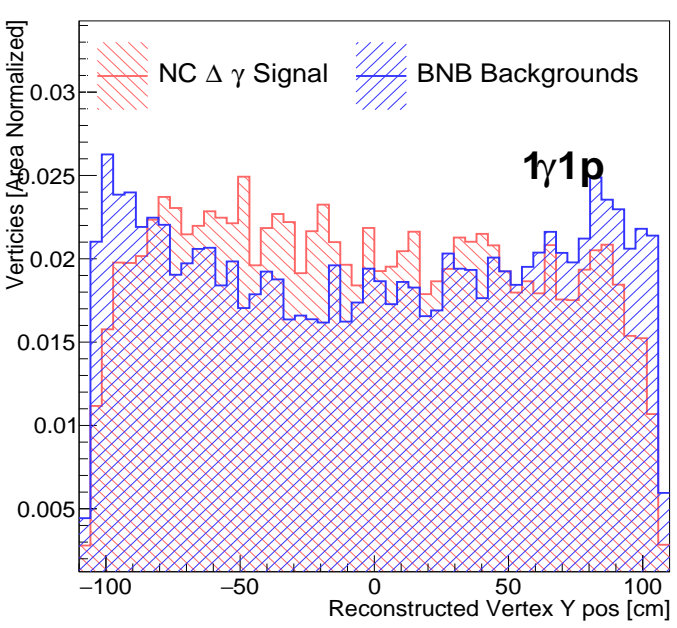

Pre-Selection Cuts

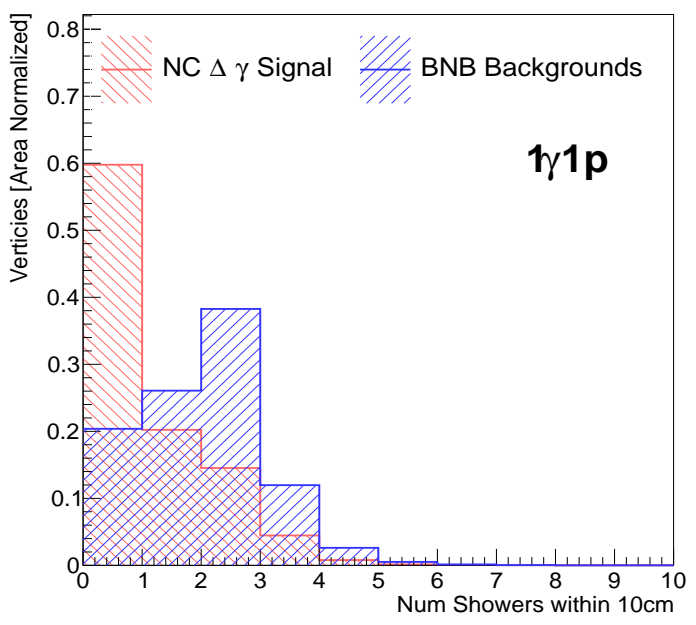

Pre-Selection Cuts

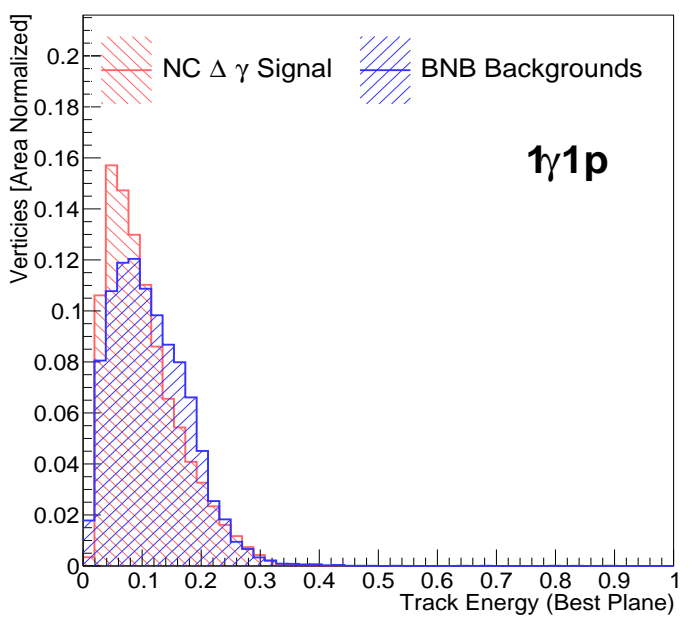

Pre-Selection Cuts

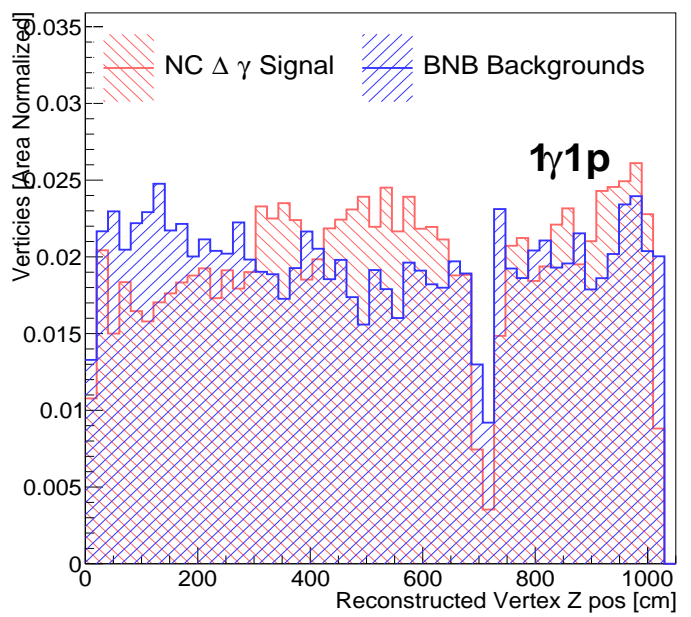

Pre-Selection Cuts

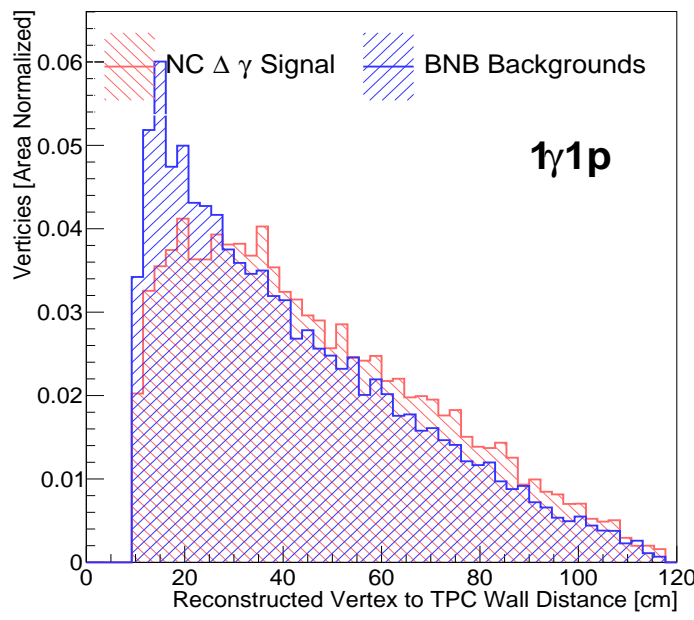

Pre-Selection Cuts

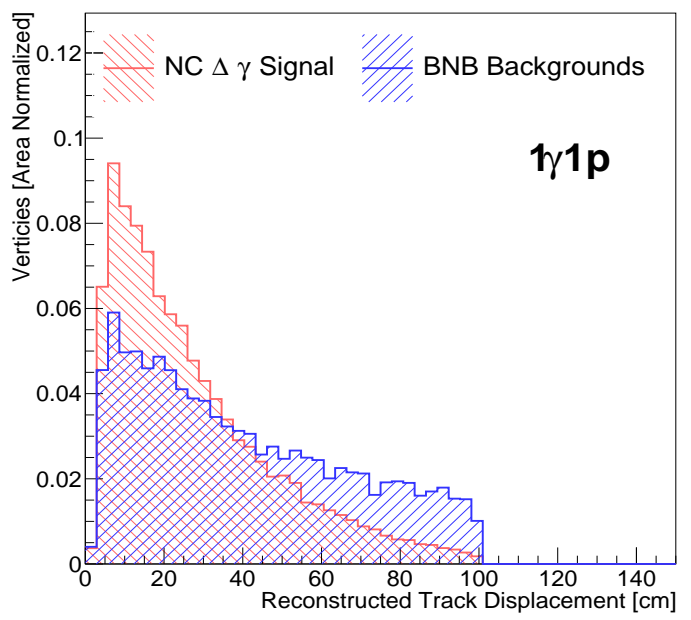

Figure 5.36: Training variables for the NC $\Delta$ radiative sample (red) and BNB sample (blue) used to train the $1 \gamma 1 p$ BNB rejection BDT. From top left to bottom right: vertex $y$-position, vertex $z$-position, number of showers within $10 \mathrm{~cm}$ of vertex, vertex-closest TPC wall distance, track energy and track length. 
Pre-Selection Cuts

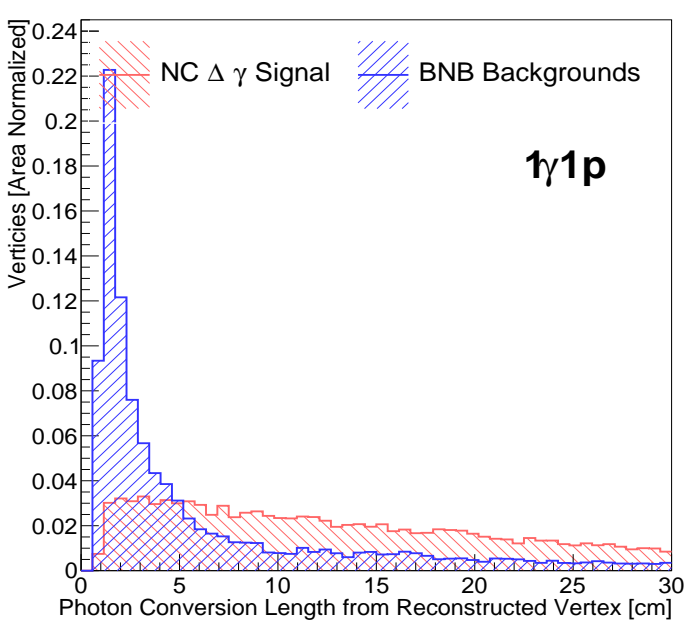

Pre-Selection Cuts

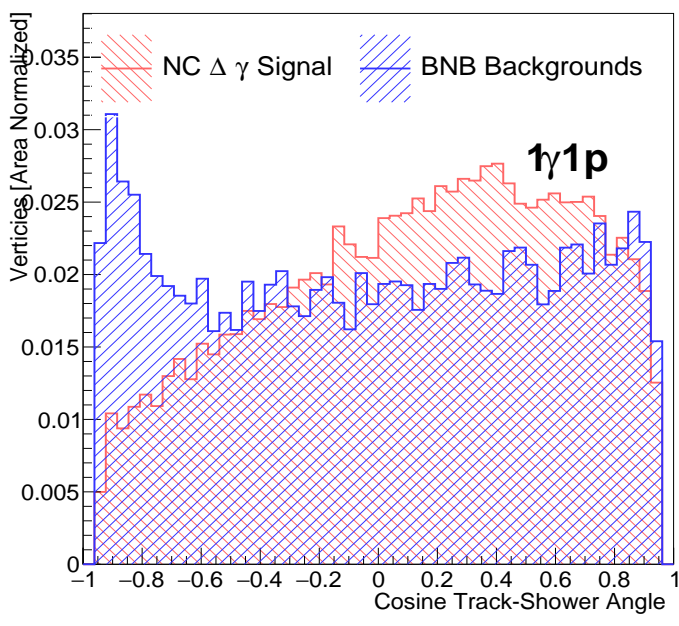

Pre-Selection Cuts

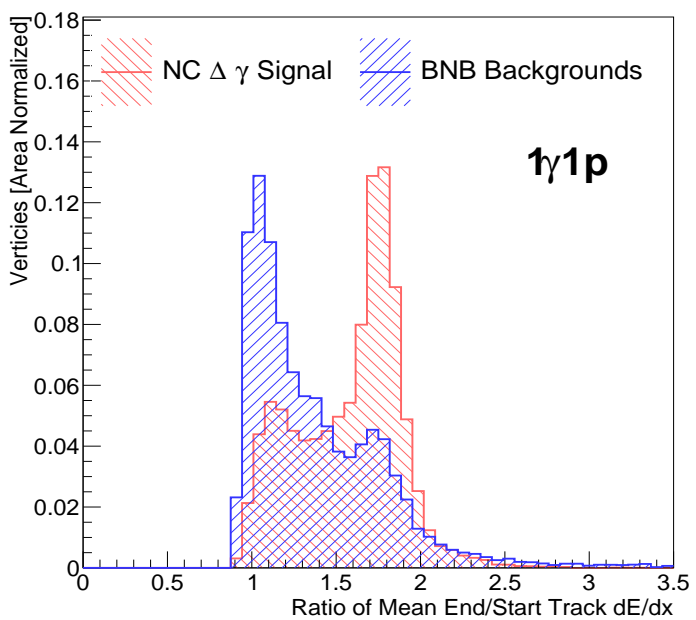

Pre-Selection Cuts

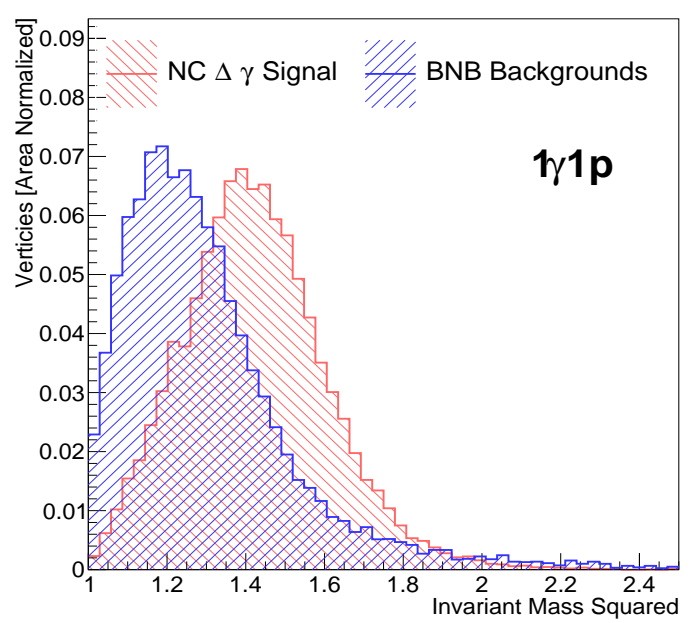

Pre-Selection Cuts

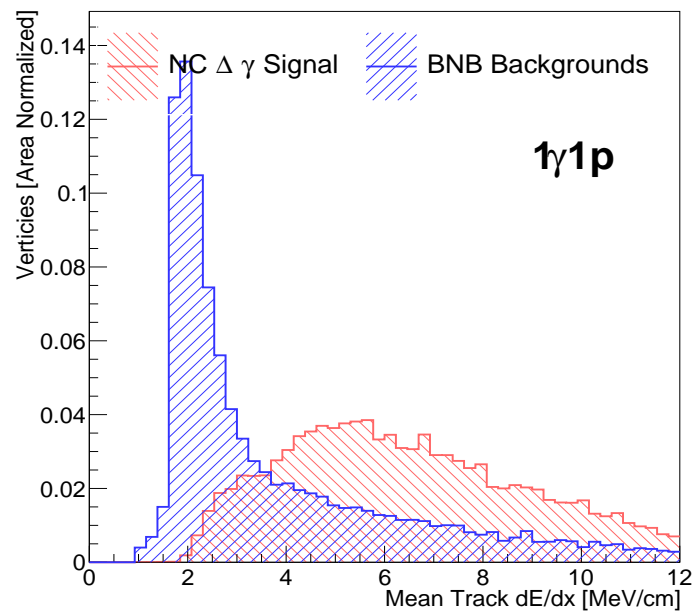

Pre-Selection Cuts

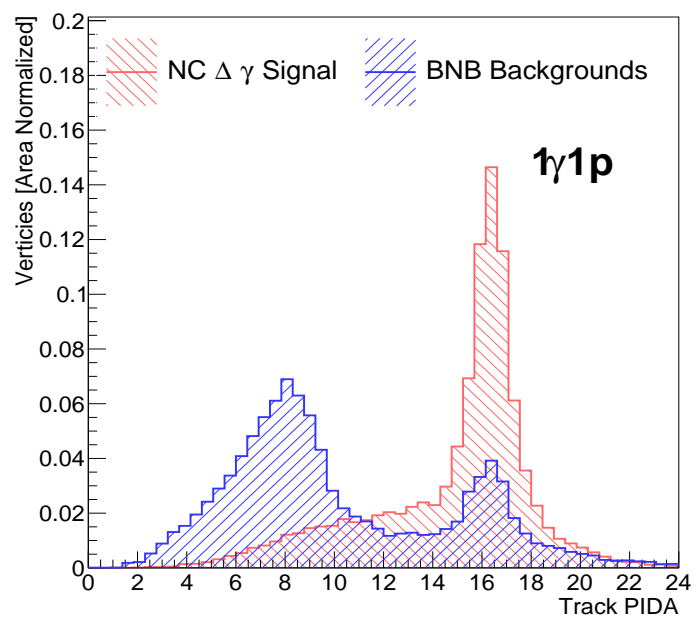

Figure 5.37: Training variables for the NC $\Delta$ radiative sample (red) and BNB sample (blue) used to train the $1 \gamma 1 p$ BNB rejection BDT. From top left to bottom right: shower start-vertex distance, track-shower invariant mass squared, cosine track-shower angle, mean track $\mathrm{dE} / \mathrm{dx}$, ratio of end-start mean track $\mathrm{dE} / \mathrm{dx}$ and track PIDA. 
Pre-Selection Cuts

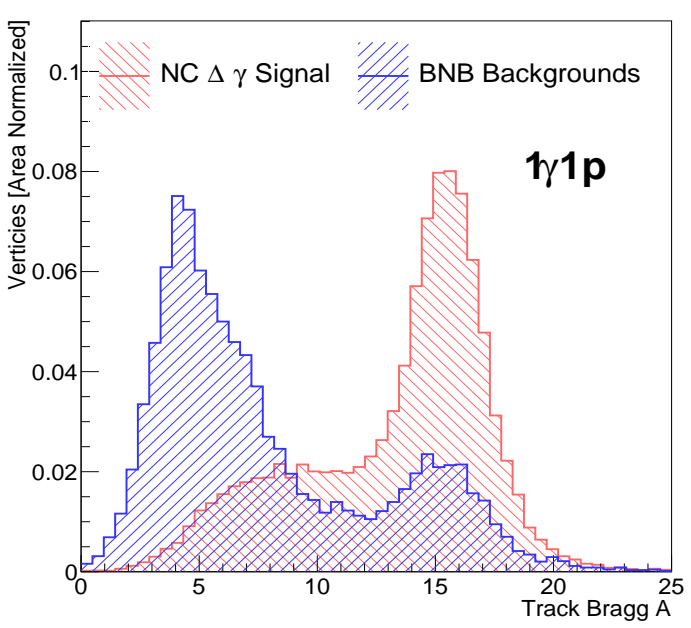

Pre-Selection Cuts

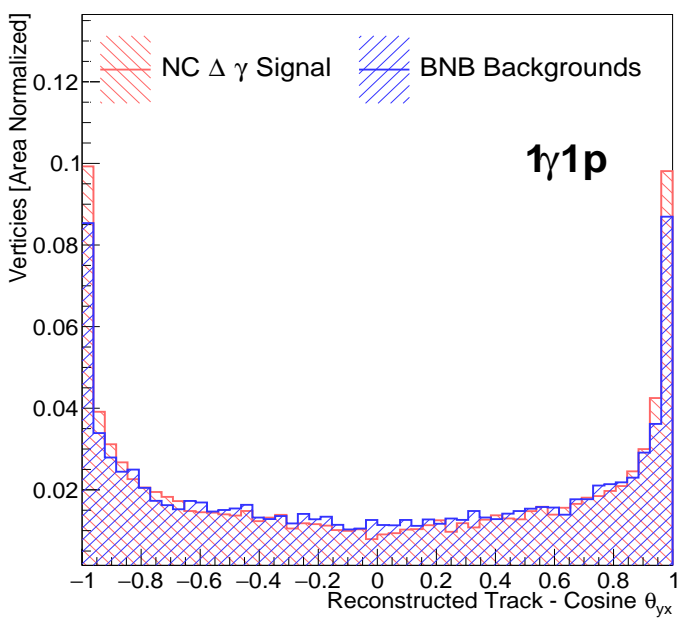

Pre-Selection Cuts

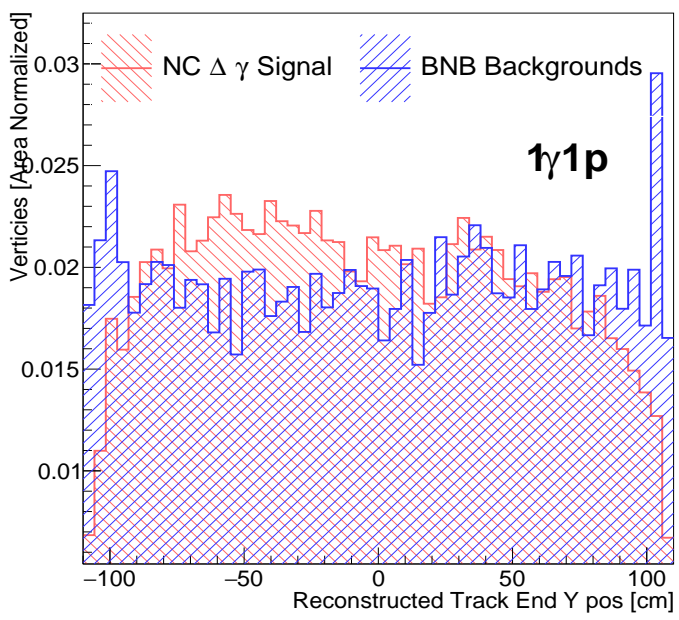

Pre-Selection Cuts

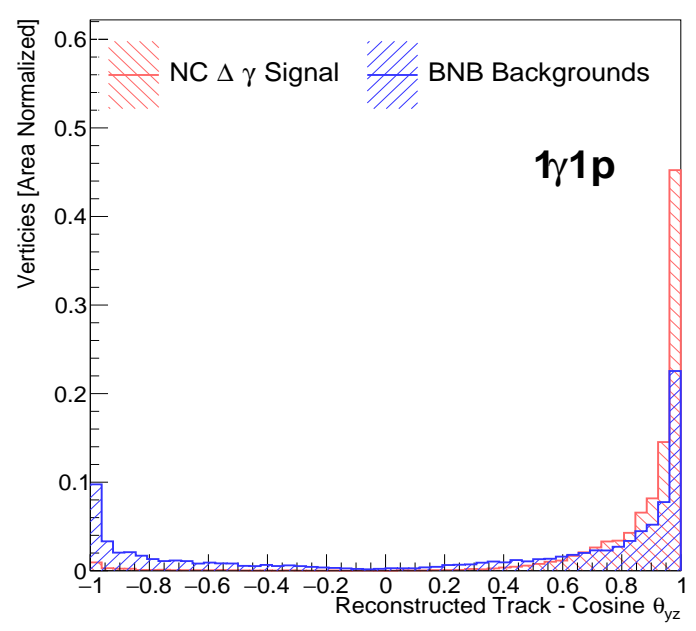

Pre-Selection Cuts

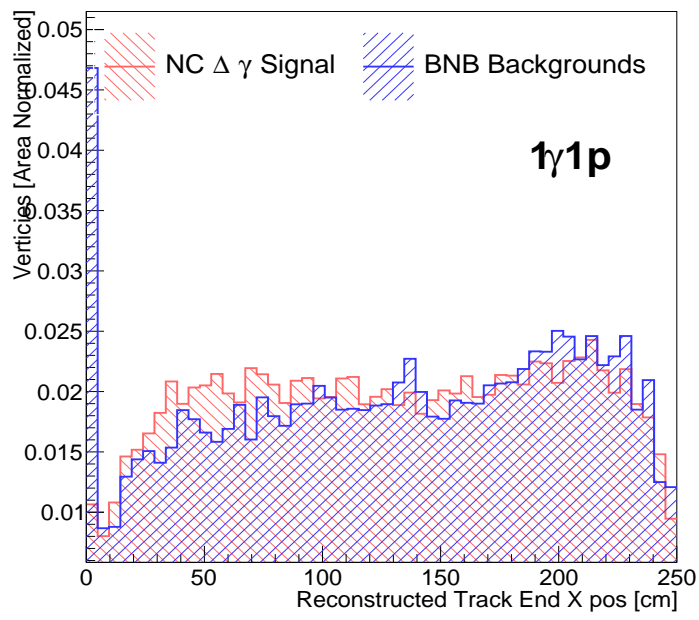

Pre-Selection Cuts

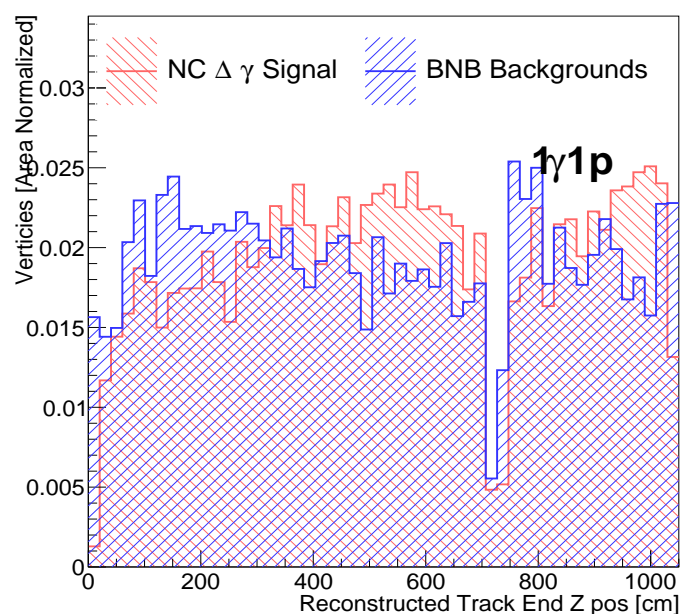

Figure 5.38: Training variables for the NC $\Delta$ radiative sample (red) and BNB sample (blue) used to train the $1 \gamma 1 p$ BNB rejection BDT. From top left to bottom right: track Bragg A value, track $\cos \theta_{y z}$, track $\cos \theta_{y x}$, track end $x$-position, track end $y$-position and track end $z$-position. 


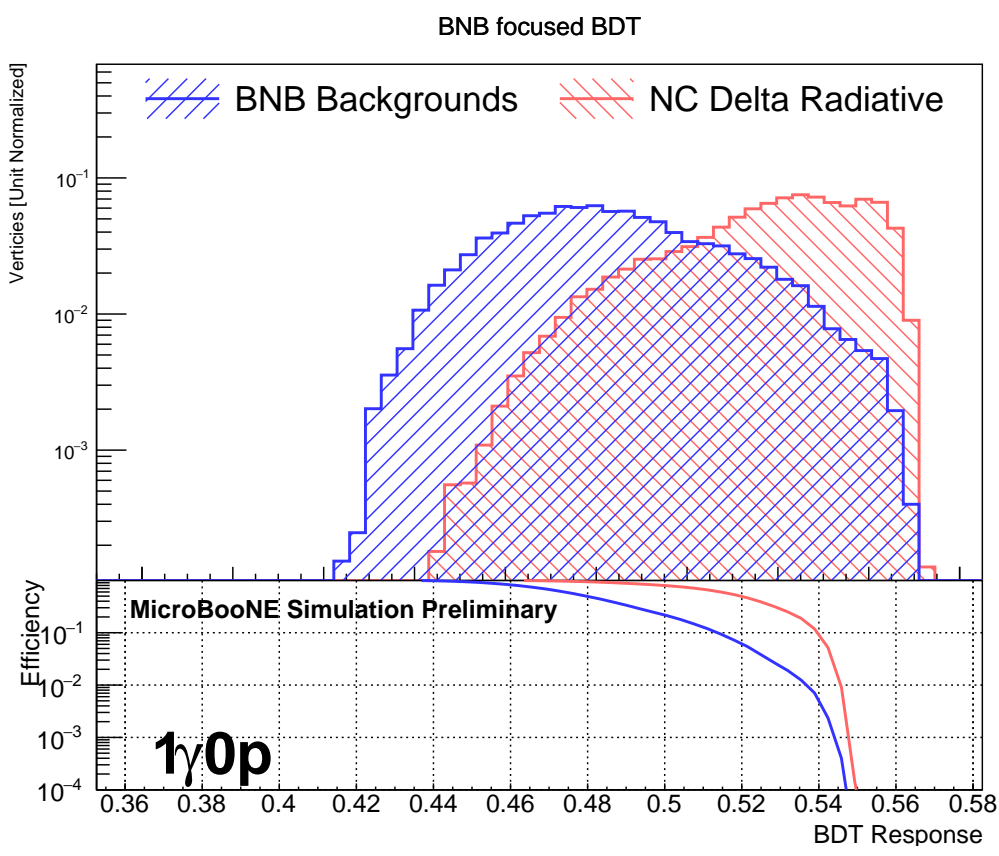

Figure 5.39: BNB rejection BDT response for the $1 \gamma 0 p$ topology. The top panel shows the area-normalized responses of the BDT to the NC $\Delta$ radiative reco-vertices (red) and BNB background vertices (blue). The more signal-like a reco-vertex is considered to be the higher the response value will be. This is can be seen in the background and signal response distributions which are shifted to the left and right respectively. The bottom panel shows the effect of placing a BDT response cut on the efficiency of each sample.

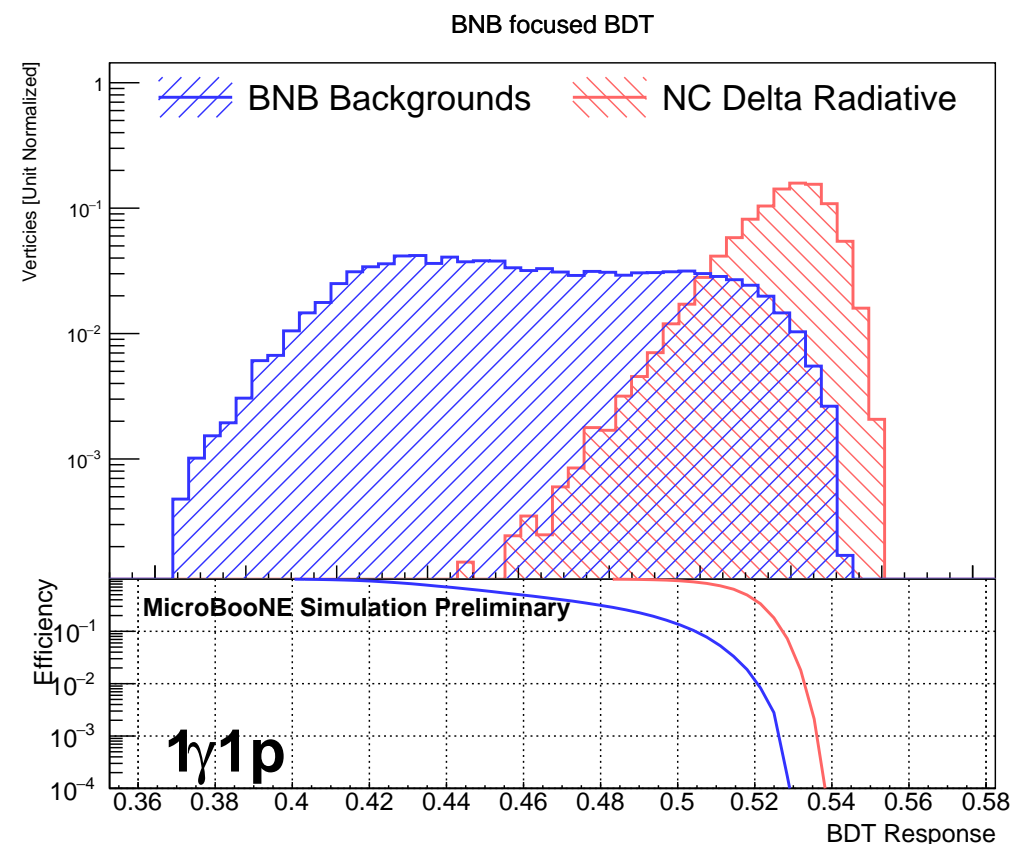

Figure 5.40: BNB rejection BDT response for the $1 \gamma 1 p$ topology. Displayed in the same manner as Figure 5.39. 


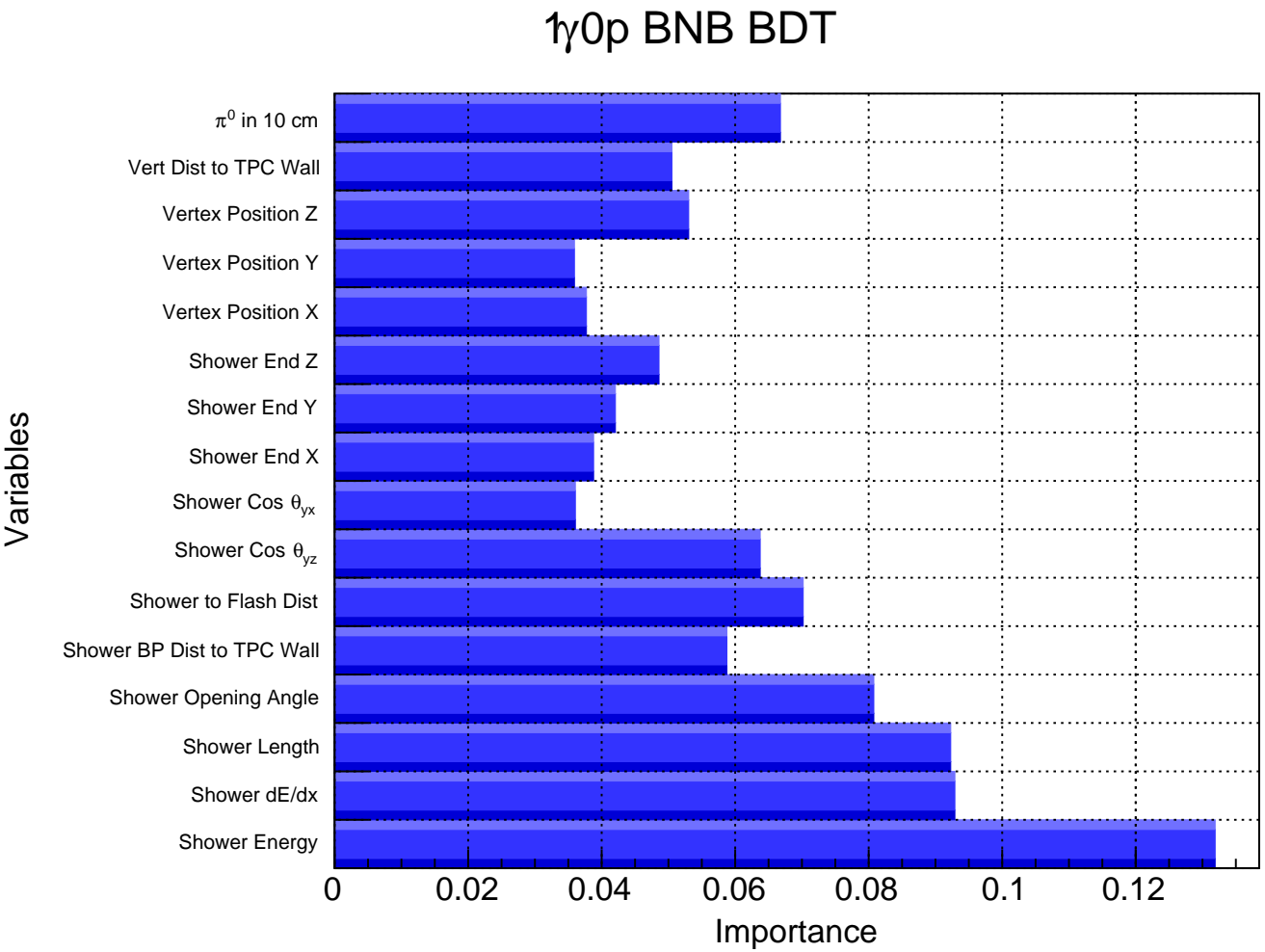

Figure 5.41: Variable importance ranking for the $1 \gamma 0 p$ BNB rejection BDT.

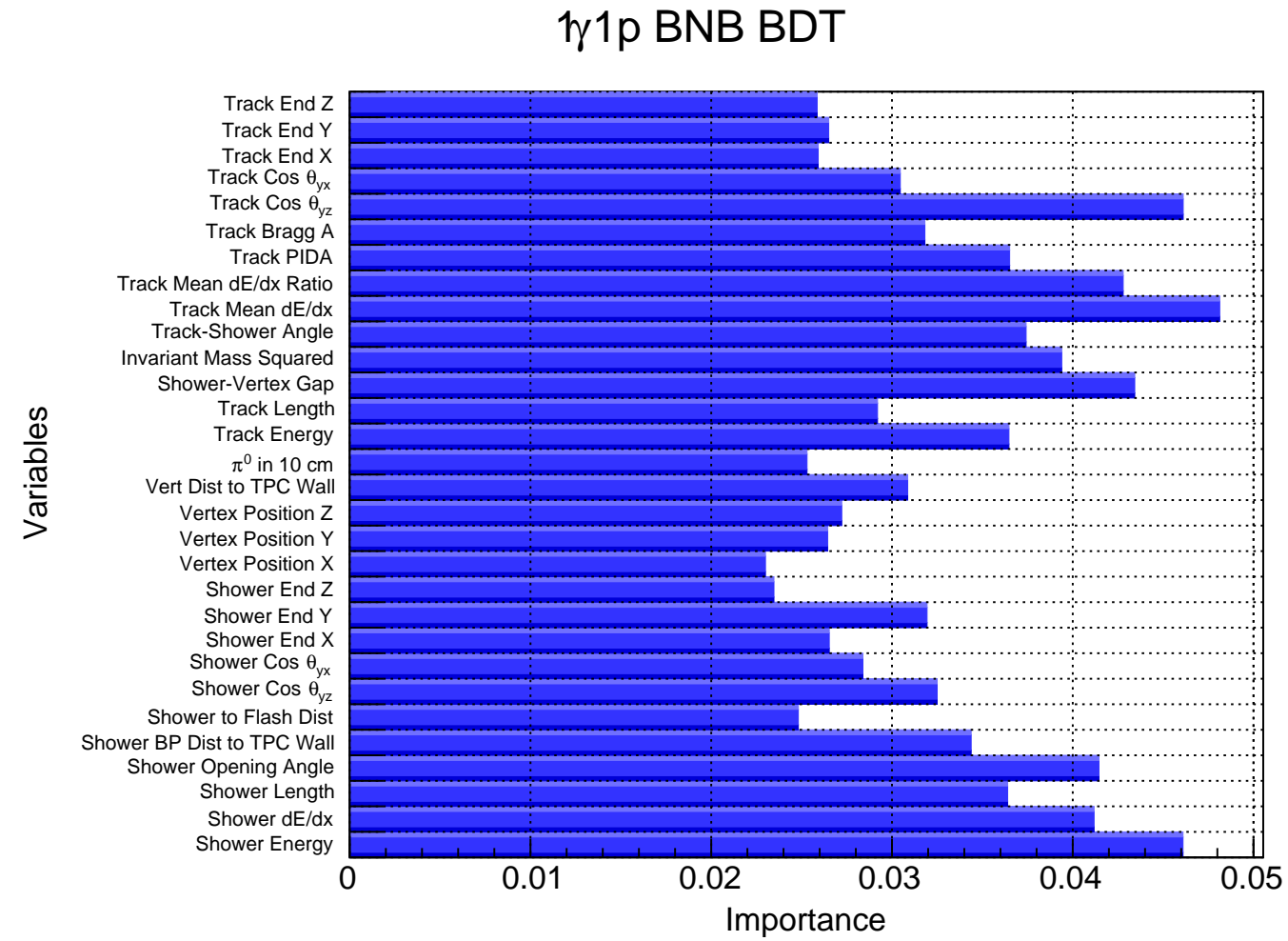

Figure 5.42: Variable importance ranking for the $1 \gamma 1 p$ BNB rejection BDT. 


\subsection{Training Variable Correlations}

Figure 5.43 shows the NC $\Delta$ radiative linear correlation coefficients of the variables used to train the $1 \gamma 1 p$ cosmic and BNB rejection BDTs. These correlation plots also effectively include the correlations for the $1 \gamma 0 p$ topology in the first sixteen shower variables on each axis. There are a large number of correlations between variables, the most significant correlations seen between variables in the $\mathrm{NC} \Delta$ radiative sample are as discussed here:

- Track energy and track length: The energy of a track will dictate the distance it is able to travel through the TPC whilst ionising liquid argon, thus a strong correlation is seen between track energy and length.

- Shower energy and length: The amount of argon an incident shower is able to ionise is dependent on the energy of that particle.

- Vertex positions $x, y$ and $z$, shower end $x, y$ and $z$ and track end $\boldsymbol{x}, \boldsymbol{y}$ and $\boldsymbol{z}$ : Vertex position, shower end and track end are all significantly correlated with each other on each axis. This is to be expected as the end of a shower or track is constrained by its start and thus the position of the vertex.

- Invariant mass, track and shower energy: The track and shower energy variables are used to directly calculate the invariant mass for the $1 \gamma 1 p$ thus the invariant mass is correlated with both.

- Track Bragg $A$, track PIDA, mean track $\mathrm{dE} / \mathrm{dx}$ ratio and mean track dE/dx: These variables are correlated with each other as $\mathrm{dE} / \mathrm{dx}$ track information is used to calculate them. In particular track bragg $A$ and track PIDA, the two variable that determine proton likelihood are highly correlated for the NC $\Delta$ radiative sample as the vast majority of tracks are true protons.

- Mean track $\mathbf{d E} / \mathbf{d x}$ and track length: Mean track $\mathrm{dE} / \mathrm{dx}$ is determined by summing $\mathrm{dE} / \mathrm{dx}$ values along the length of the track and then dividing that sum by the length to find the average. As a result, mean track $d E / d x$ and length are be anti-correlated. Strong correlation between track length and track energy also result in correlation between mean track $\mathrm{dE} / \mathrm{dx}$ and track energy.

Similar correlations can be seen for the in-time cosmic and BNB background samples shown in Figures 5.44 and 5.45, though some of the correlations between variables in the NC $\Delta$ radiative sample are less pronounced for the background samples. In particular there is significantly less correlation between the track Bragg $A$, track 
PIDA, mean track $\mathrm{dE} / \mathrm{dx}$ ratio and mean track $\mathrm{dE} / \mathrm{dx}$ variables for the background samples, due to the lower number of proton tracks.

\section{$1 \gamma 1 \mathrm{p}$ NC $\Delta$ Radiative}

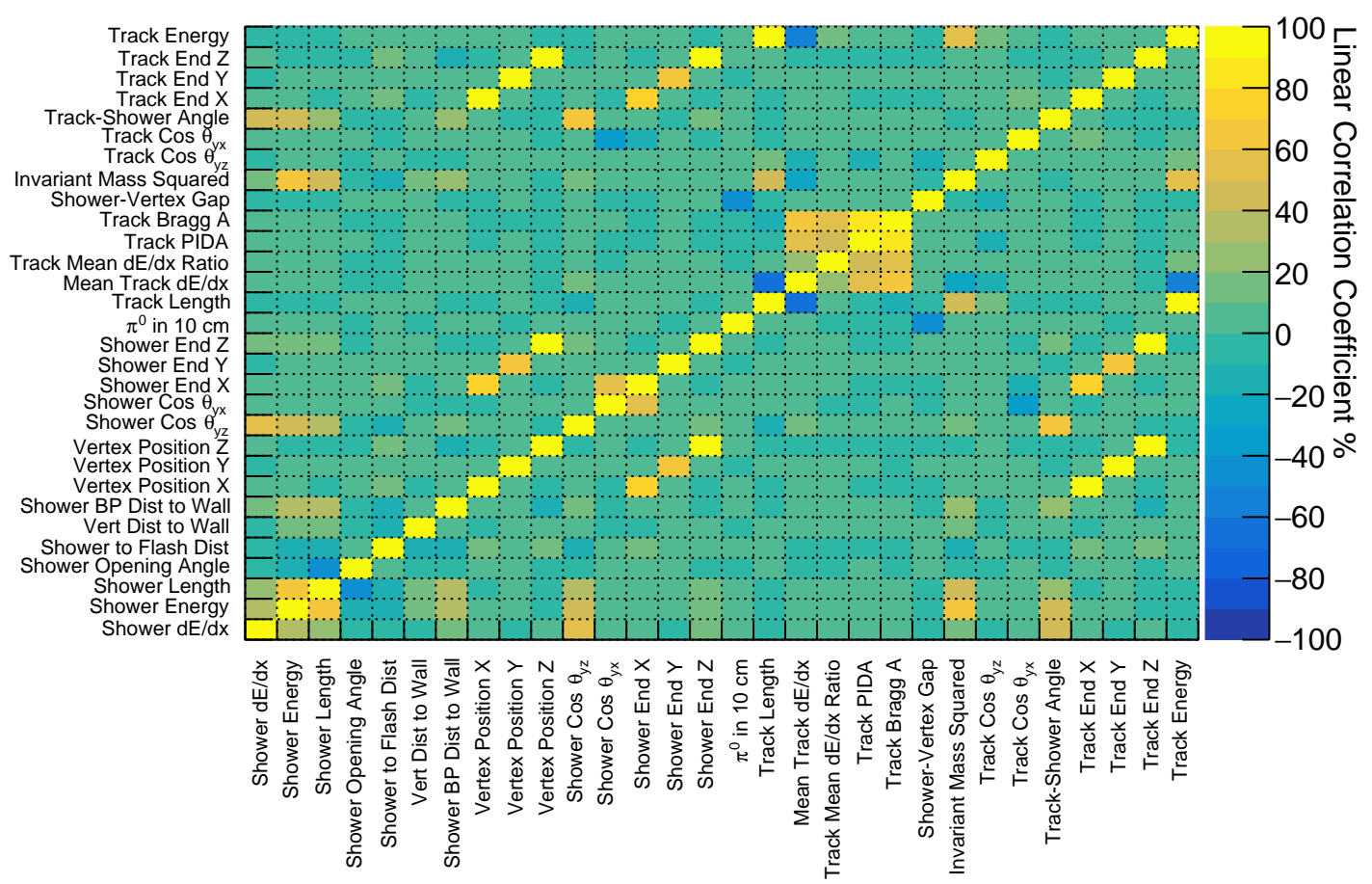

Figure 5.43: $1 \gamma 1 p$ NC $\Delta$ radiative training variable linear correlation coefficients. 


\section{$1 \gamma 1 \mathrm{p}$ In-time cosmic}
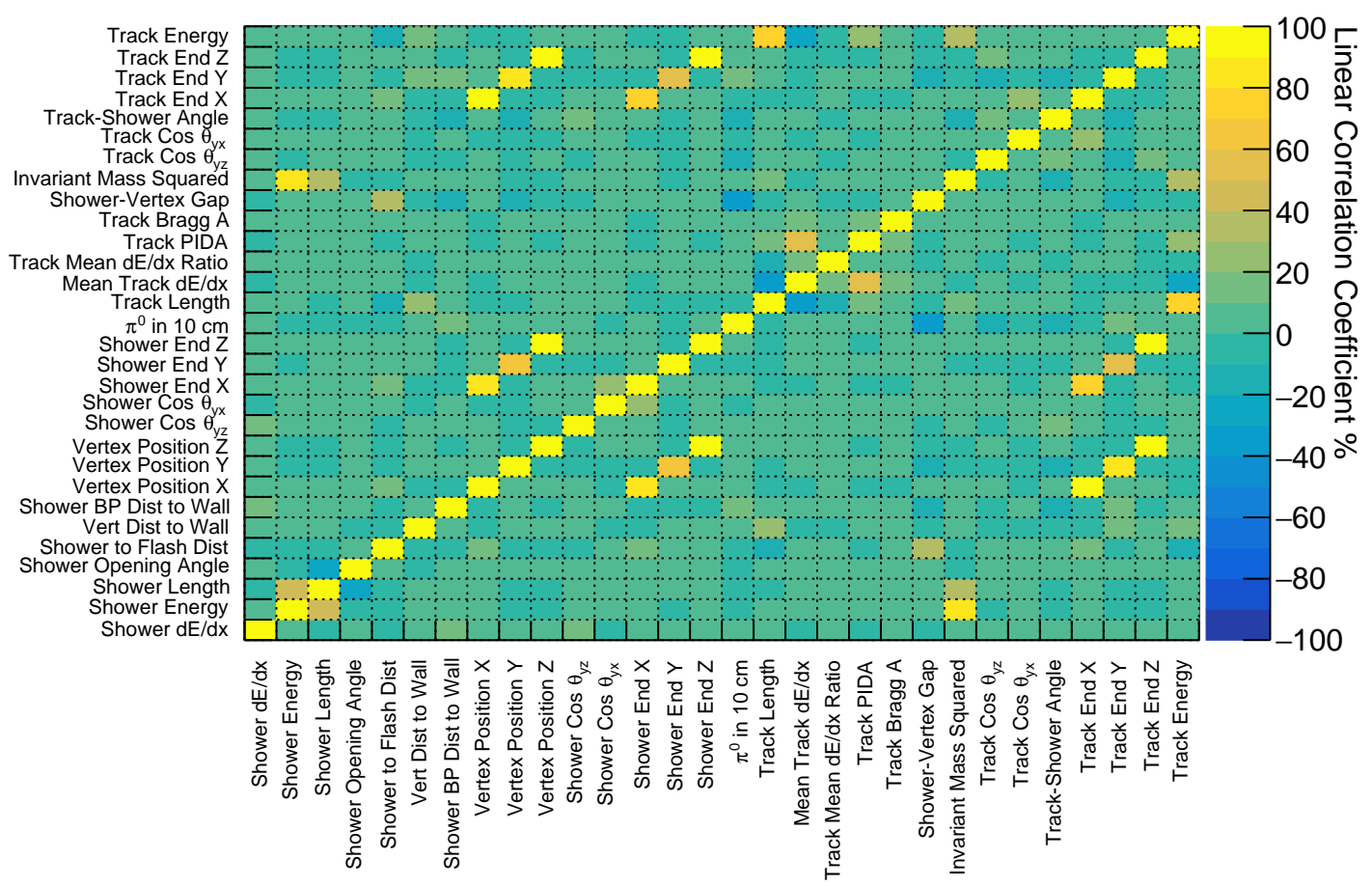

Figure 5.44: $1 \gamma 1 p$ in-time cosmic training variable linear correlation coefficients.

\section{$1 \gamma 1 p$ BNB Background}

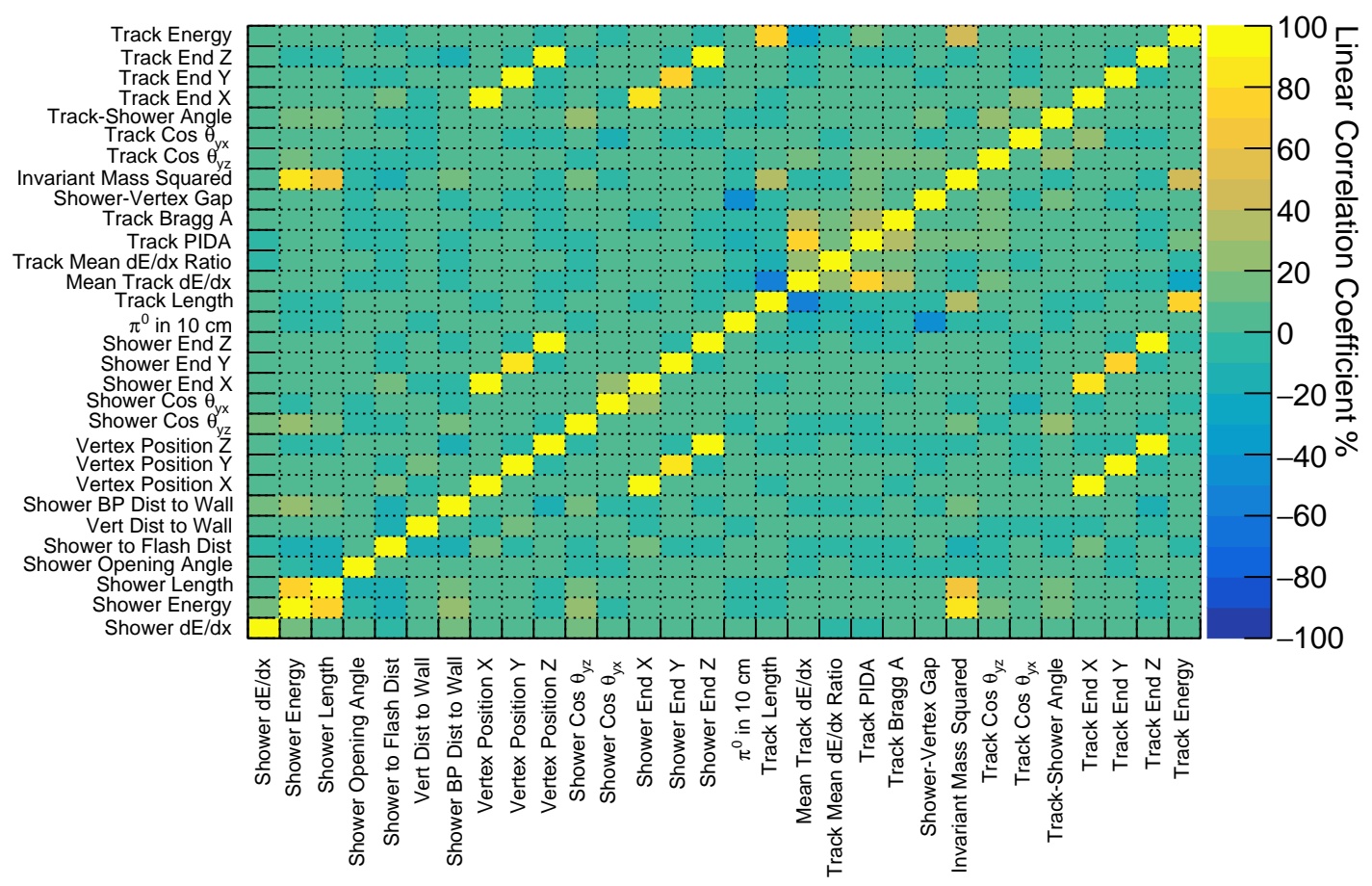

Figure 5.45: $1 \gamma 1 p$ BNB training variable linear correlation coefficients. 


\subsubsection{Removal of Correlated Variables}

In order to test the impact of the correlated variables on the performance of the cosmic and BNB background rejection BDTs, significantly correlated variables were removed and the BDTs were re-trained on the remaining variables. The following variables were removed:

- Shower length $(\mathrm{cm})$

- Shower end point in $x, y, z(\mathrm{~cm})$

- Shower cosine $\theta_{y z}$

- Invariant mass squared of track-shower pair assuming a photon shower and proton track $\left(\mathrm{GeV}^{2}\right)$

- Cosine of the track-shower opening angle

- Track energy (GeV)

- Track end point in $x, y, z(\mathrm{~cm})$

- Track mean $d E / d x(\mathrm{MeV} / \mathrm{cm})$

- Ratio of the mean $d E / d x$ of the first half of the track to the second half

- Track proton PIDA (particle identification A)

- Track Bragg-fit parameter $A$

Figures 5.46 and 5.47 show the cosmic BDT performance for both $1 \gamma 0 p$ and $1 \gamma 1 p$ topologies resulting from the training on the reduced-correlations variable list. Cosmic BDT separation for both topologies is marginally worse with the reducedcorrelations variable list, compared to the full list. Figures 5.48 and 5.49 show the cosmic BDT variable importance rankings for both $1 \gamma 0 p$ and $1 \gamma 1 p$ topologies. The addition or removal of BDT training variables can cause the importance ranking of pre-existing variables to change. For example the importance of a variable already used in a BDT may increase due to the introduction of a new variable which results in the formation of additional decision nodes in the BDT, allowing it to place more powerful cuts using the pre-existing variable. In the case of the $1 \gamma 0 p$ and $1 \gamma 1 p$ cosmic BDTs, the variable importance rankings using the full and reduced-correlations variable list remain mostly the same.

Figures 5.50 and 5.51 show the BNB BDT performance for both $1 \gamma 0 p$ and $1 \gamma 1 p$ topologies resulting from the training on the reduced-correlations variable list. 
As was with the cosmic BDTs, the BNB BDT separation for both topologies is marginally worse using reduced-correlations variable list. Figures 5.52 and 5.53 show the BNB BDT variable importance rankings for both $1 \gamma 0 p$ and $1 \gamma 1 p$ topologies. Once again the variable importance rankings remain roughly the same. Given the prevalence of background and the small signal size for the analysis, the marginal decrease in performance for the cosmic and BNB BDTs trained using the reducedcorrelations variable list results in a significant decrease in sensitivity to NC $\Delta$ radiative decay for both topologies compared to the BDTs trained using the full variable list. A comparison of $\mathrm{NC} \Delta$ radiative statistical significance using the full variable list BDTs and the reduced-correlations variable list BDTs can be found in Chapter 6. Due to the decrease in sensitivity of the search using the reducedcorrelations variable list, the full variable list is used in spite of the correlations. Figures 5.54, 5.55 and 5.56 show the linear correlation coefficients of the new set of variables for the $1 \gamma 1 p$ NC $\Delta$ radiative, in-time cosmic and BNB background samples. The plots show that correlations between the variables in the reduced-correlations variable list is minimal. 


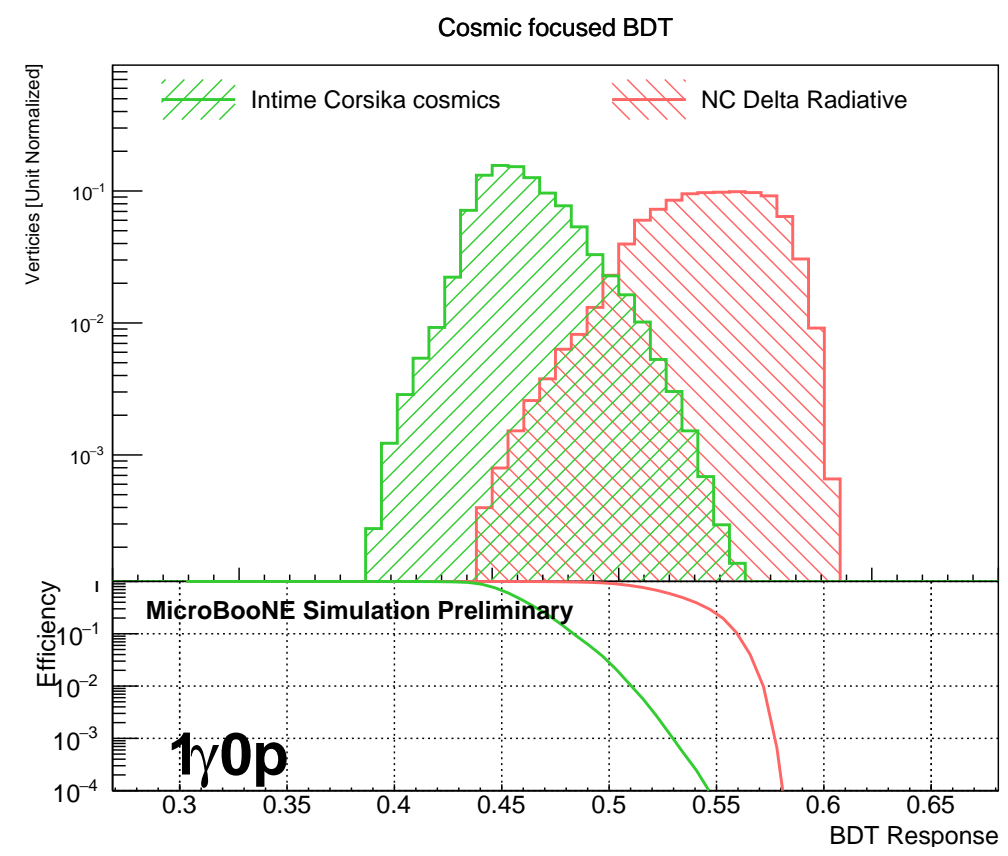

Figure 5.46: Cosmic rejection BDT response for the $1 \gamma 0 p$ topology, trained using the reduced-correlations variable list.

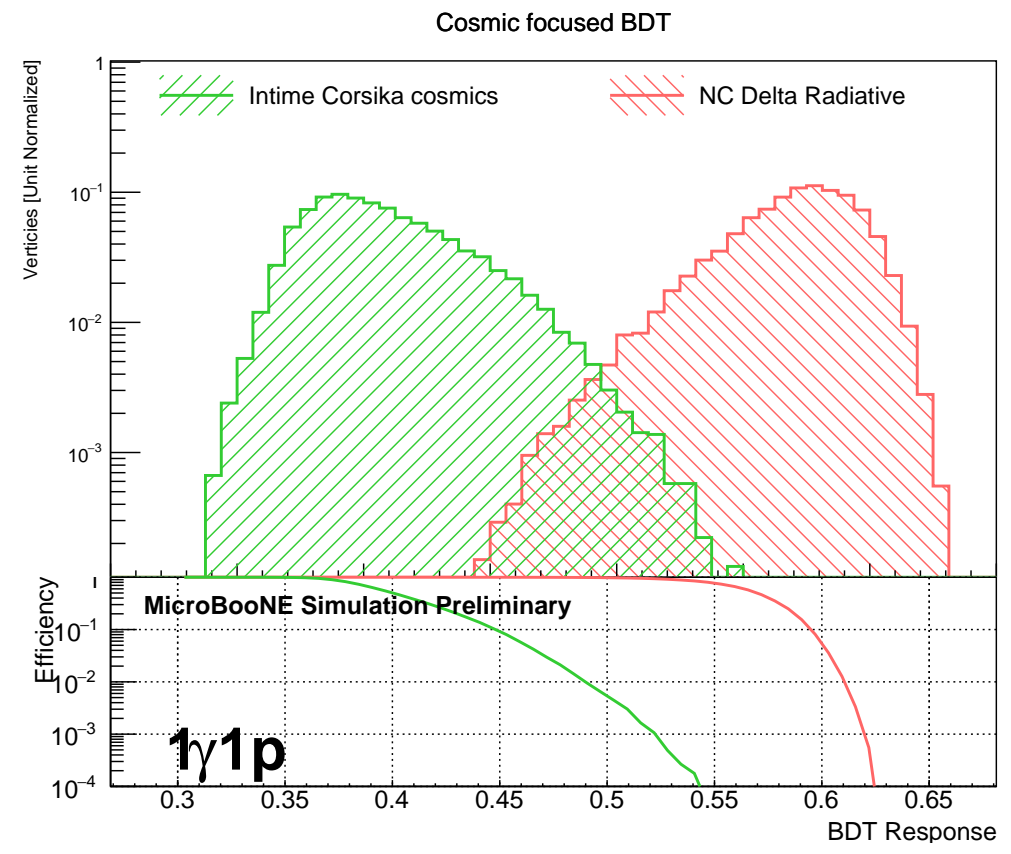

Figure 5.47: Cosmic rejection BDT response for the $1 \gamma 1 p$ topology, trained using the reduced-correlations variable list. 


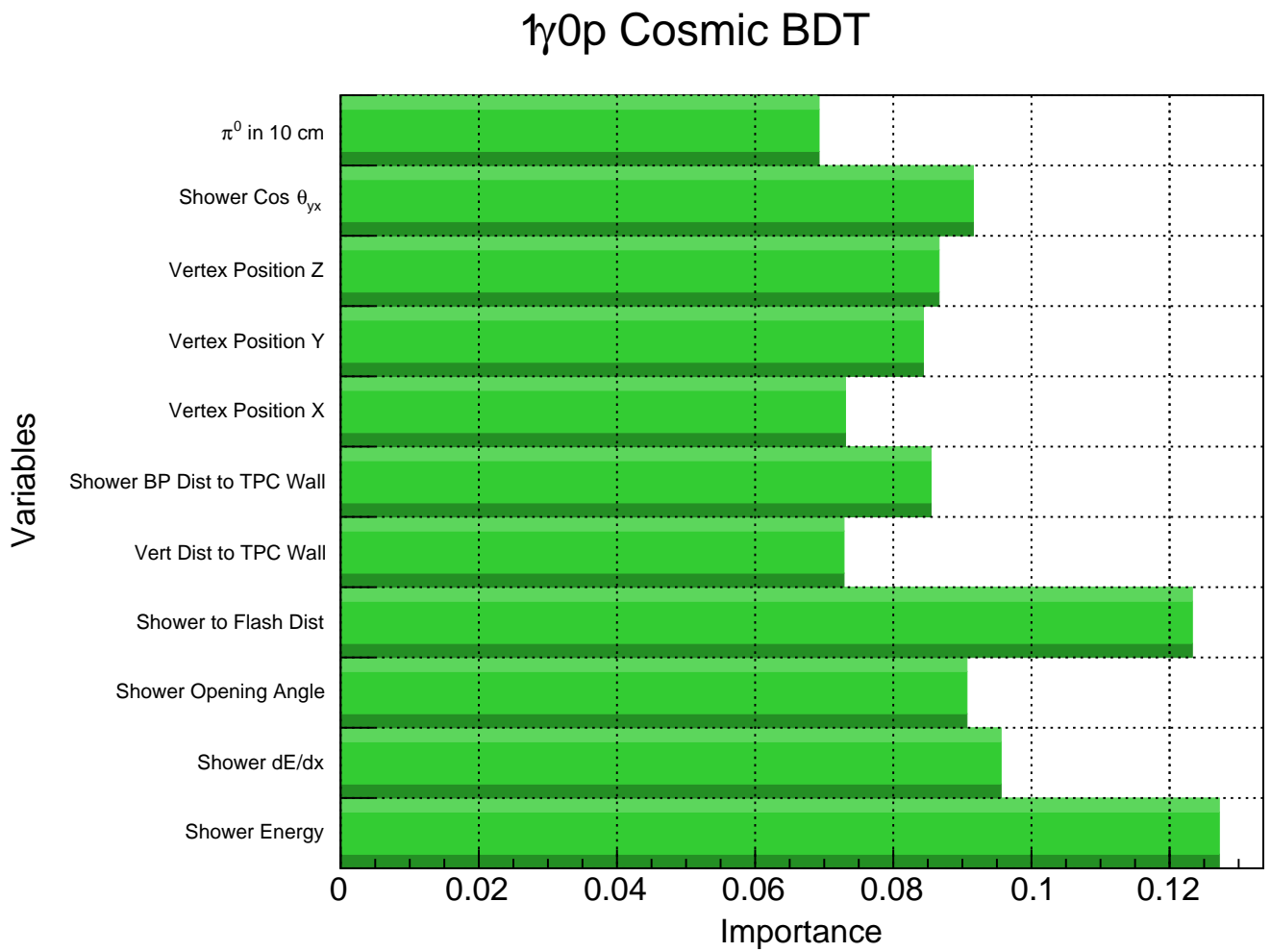

Figure 5.48: Variable importance ranking for the $1 \gamma 0 p$ cosmic rejection BDT, trained using the reduced-correlations variable list.

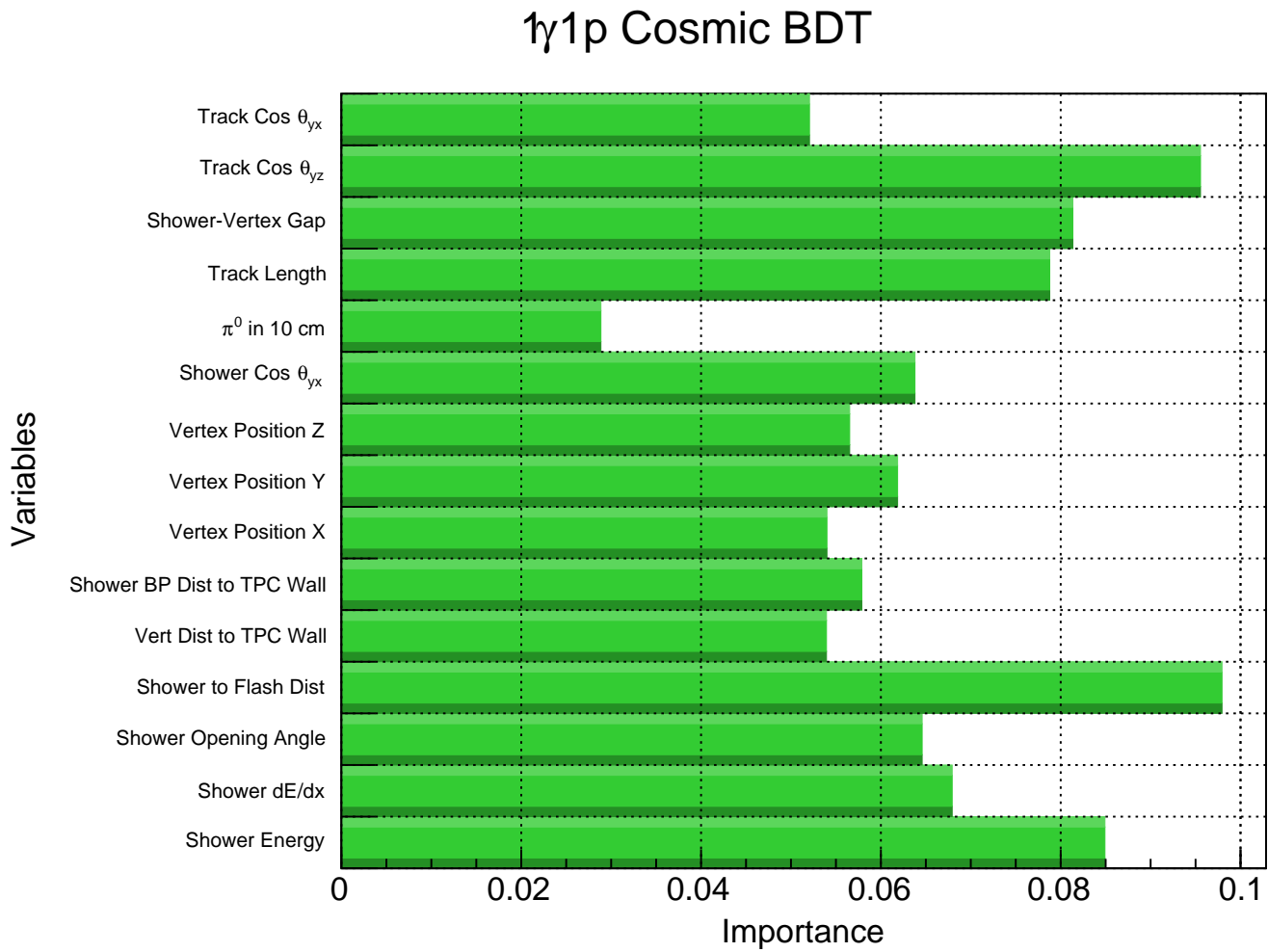

Figure 5.49: Variable importance ranking for the $1 \gamma 1 p$ cosmic rejection BDT, trained using the reduced-correlations variable list. 


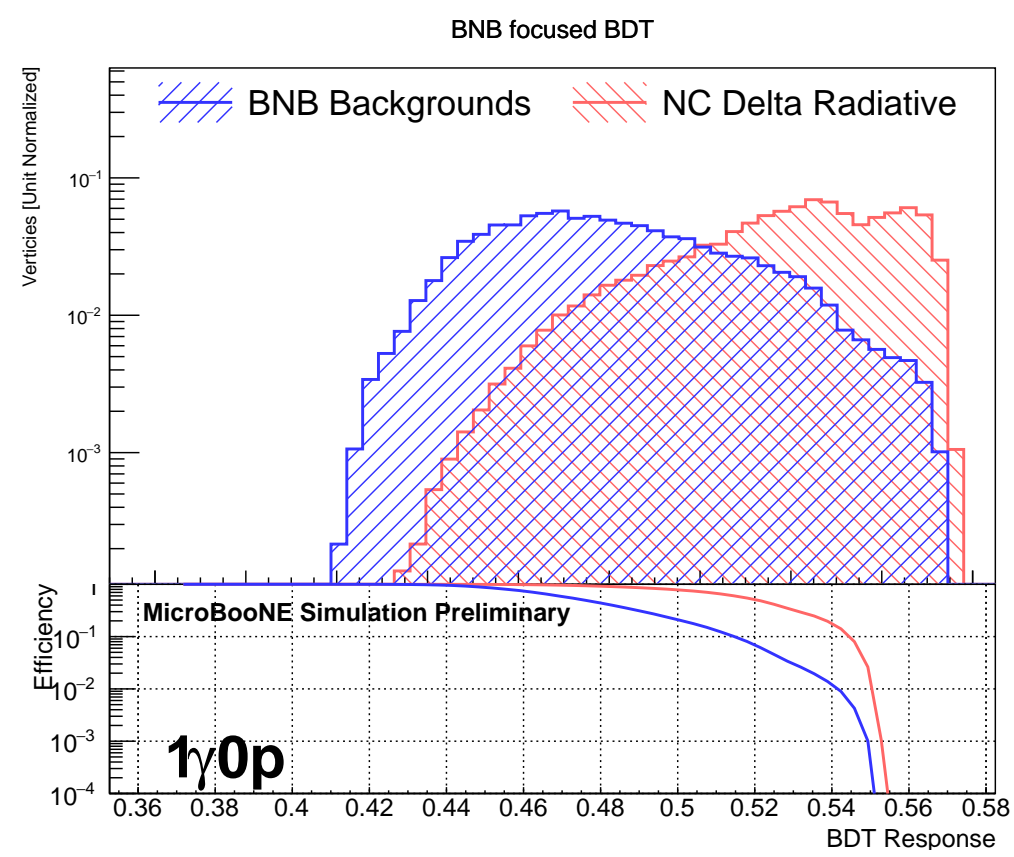

Figure 5.50: BNB rejection BDT response for the $1 \gamma 0 p$ topology, trained using the reduced-correlations variable list.

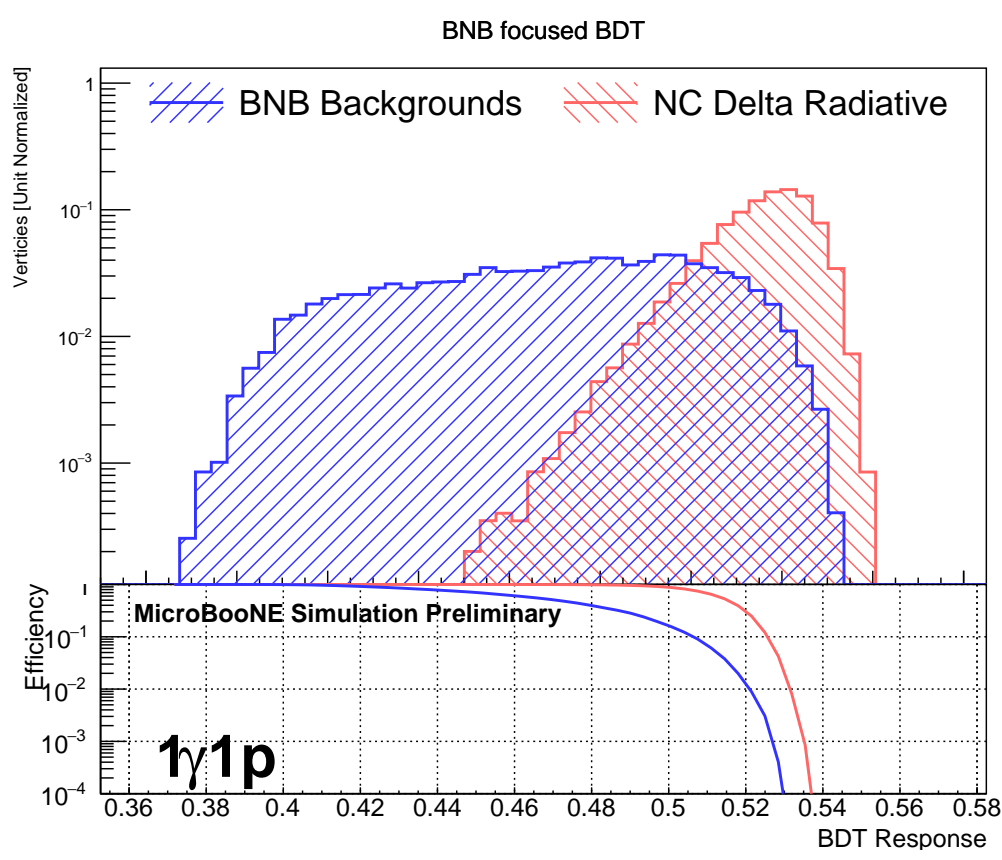

Figure 5.51: BNB rejection BDT response for the $1 \gamma 1 p$ topology, trained using the reduced-correlations variable list. 


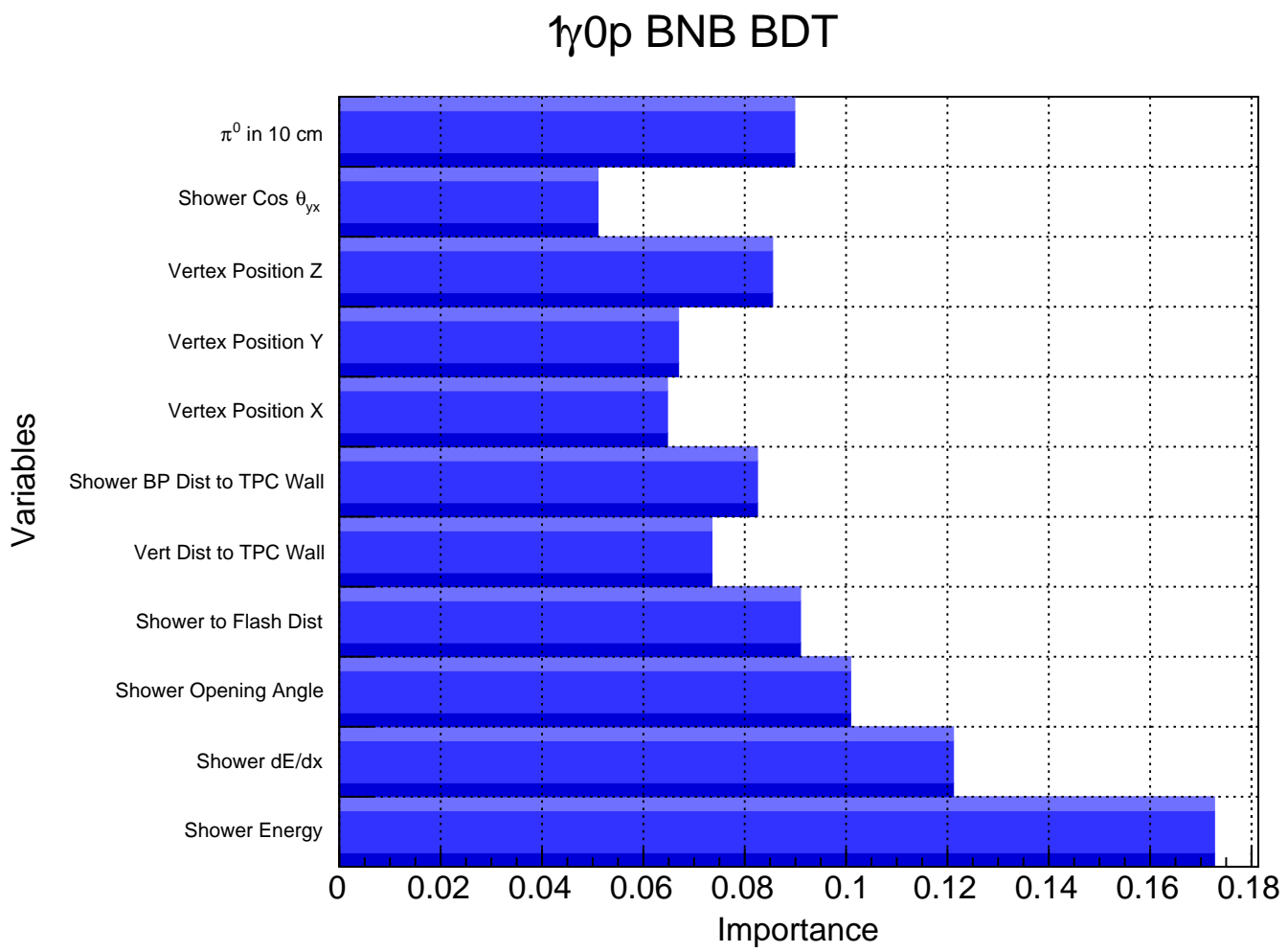

Figure 5.52: Variable importance ranking for the $1 \gamma 0 p$ BNB rejection BDT, trained using the reduced-correlations variable list.

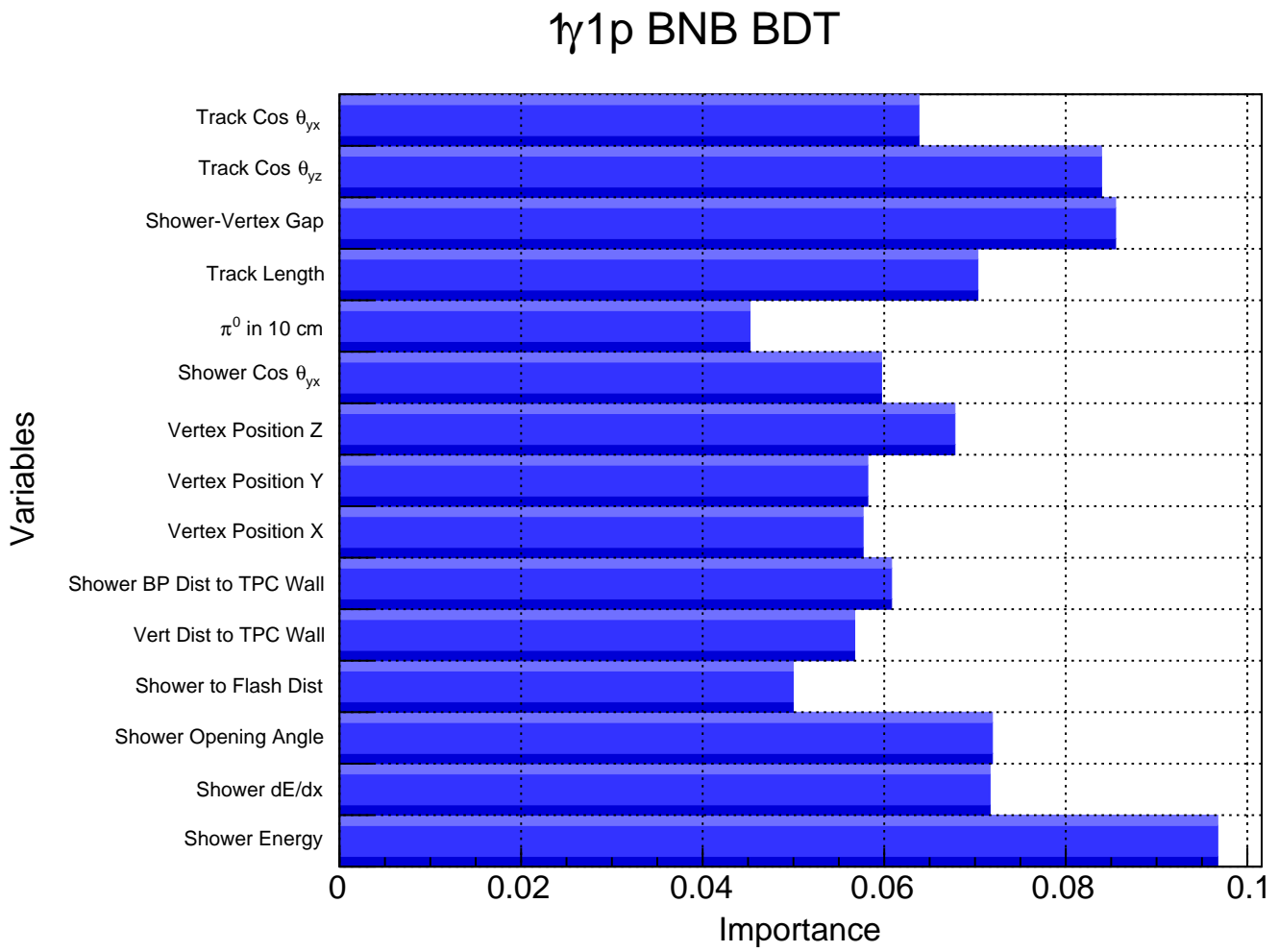

Figure 5.53: Variable importance ranking for the $1 \gamma 1 p$ BNB rejection BDT, trained using the reduced-correlations variable list. 


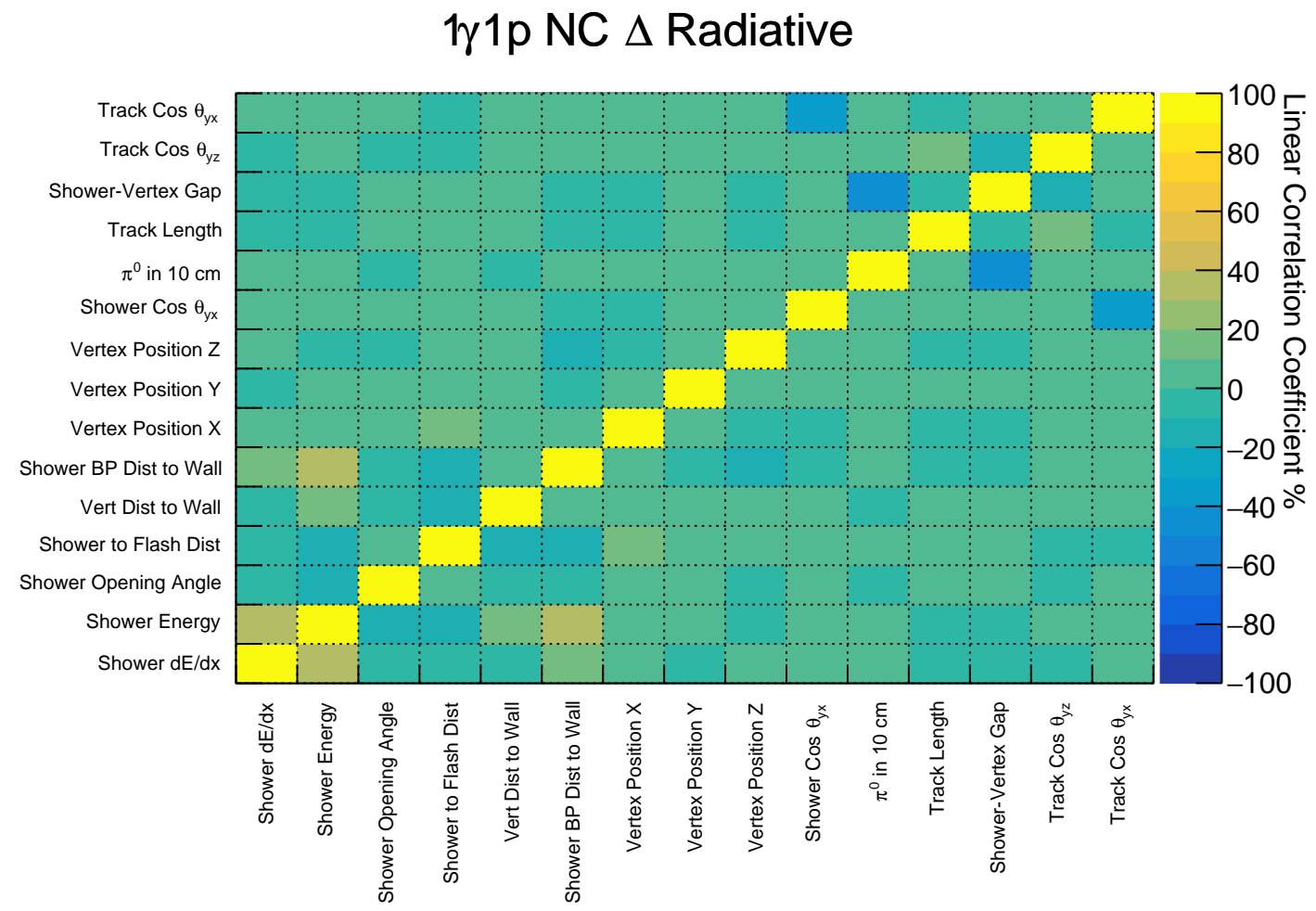

Figure 5.54: $1 \gamma 1 p$ NC $\Delta$ radiative training variable linear correlation coefficients, using the reduced-correlations variable list.

\section{$1 \gamma 1 p$ In-time cosmic}

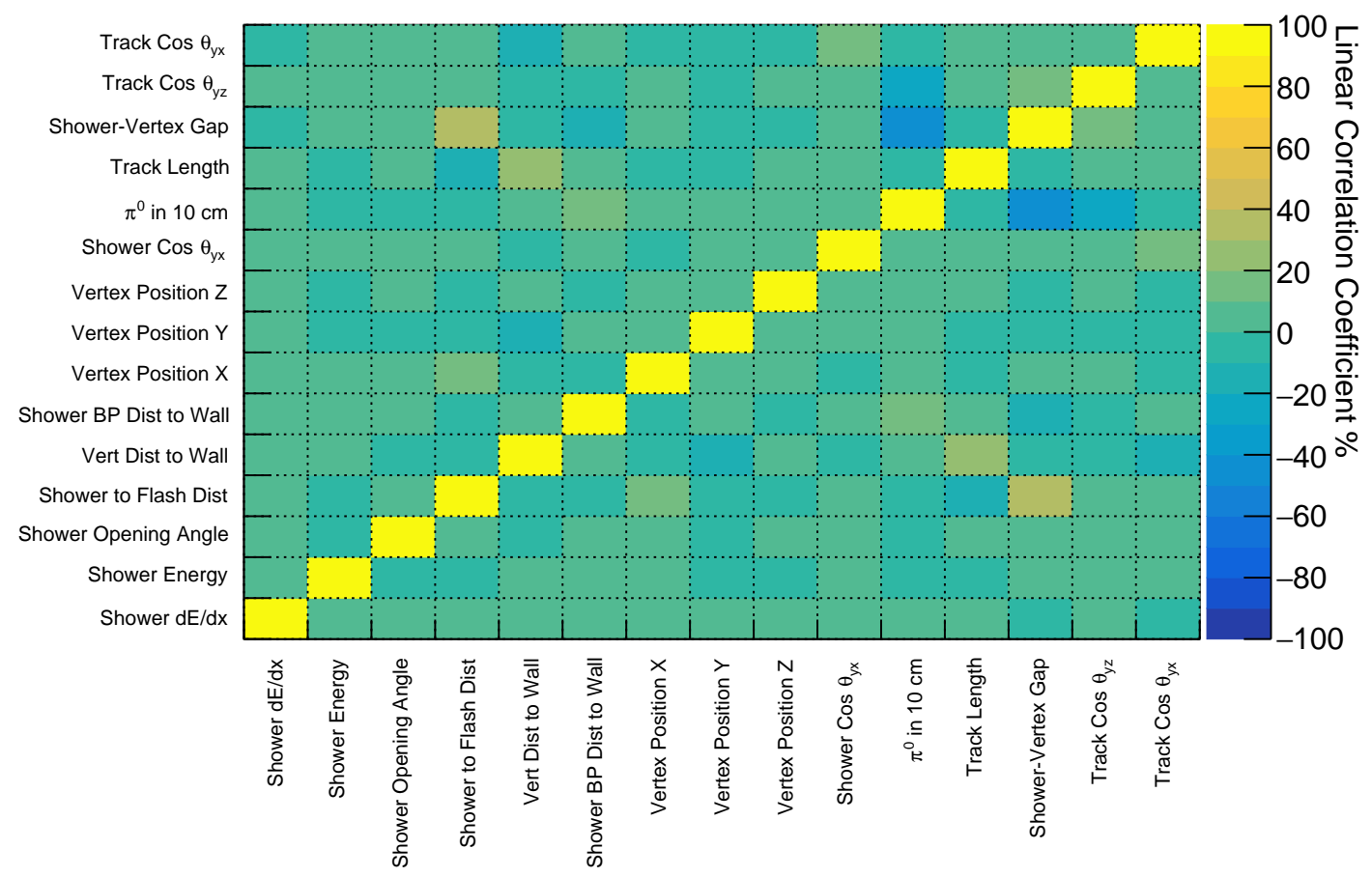

Figure 5.55: $1 \gamma 1 p$ in-time cosmic training variable linear correlation coefficients, using the reduced-correlations variable list. 


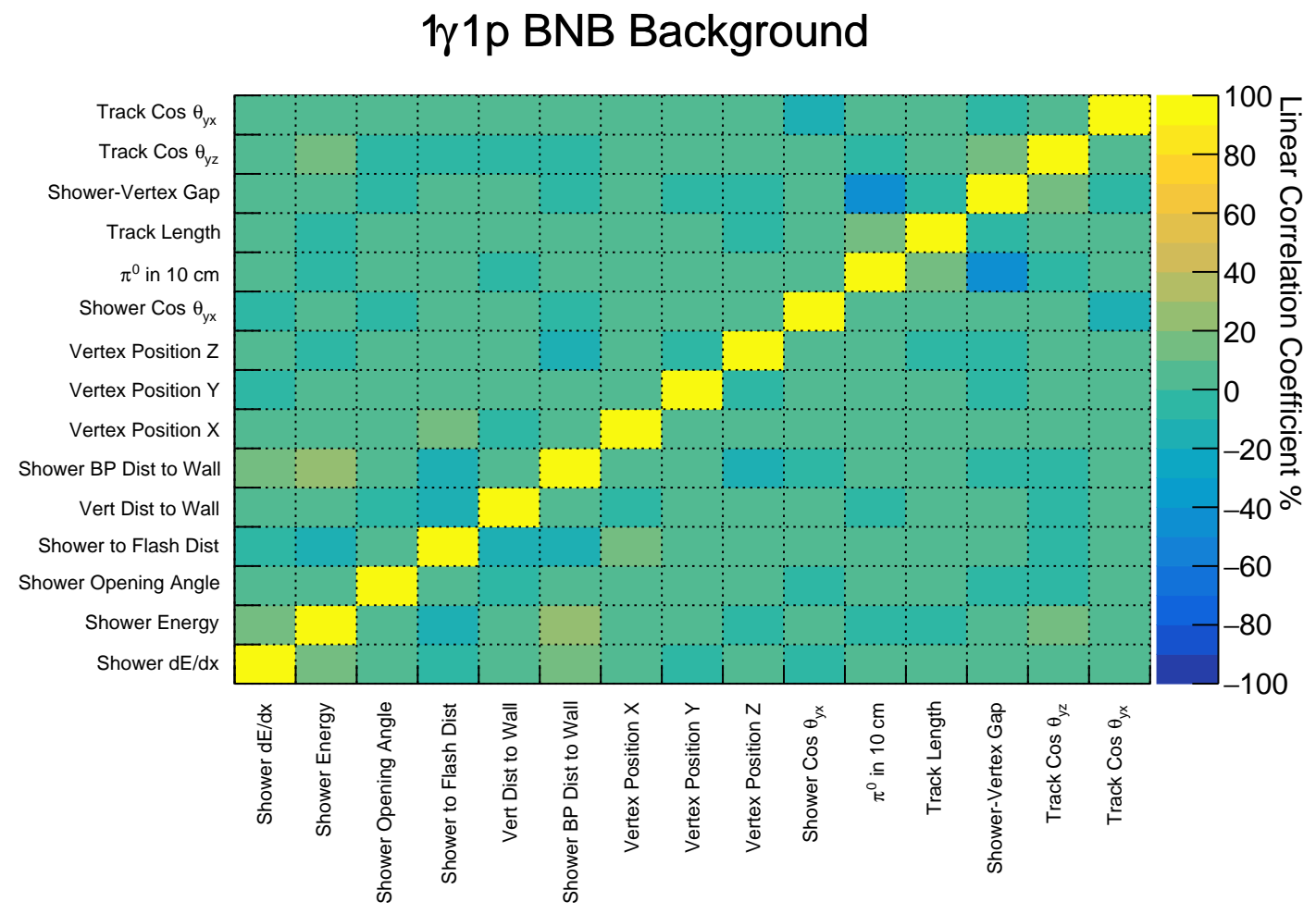

Figure 5.56: $1 \gamma 1 p$ BNB training variable linear correlation coefficients, using the reduced-correlations variable list. 


\subsection{Boosted Decision Tree Response}

To ensure acceptable training statistics for the BNB BDTs, the cosmic and BNB BDTs were trained simultaneously rather than training the cosmic BDTs first, applying response cuts and then training the BNB BDTs. BDT response cuts were selected independently for the $1 \gamma 1 p$ and $1 \gamma 0 p$ topologies. The position of the response cuts for the cosmic and BNB BDTs in each topology were chosen by simultaneously varying the cosmic and BNB BDT response cuts and selecting the combination of response cuts that maximized the statistical significance $\left(\frac{s}{\sqrt{b}}\right)$ of the NC $\Delta$ radiative signal for $6.6 \times 10^{20}$ POT. Table 5.3 shows the selected response cut value for each BDT.

\begin{tabular}{|c|c|c|}
\hline & Cosmic BDT & BNB BDT \\
\hline $1 \gamma 0 p$ & 0.541 & 0.527 \\
$1 \gamma 1 p$ & 0.547 & 0.518 \\
\hline
\end{tabular}

Table 5.3: Cosmic and BNB BDT response cut values for the $1 \gamma 0 p$ and $1 \gamma 1 p$ topologies chosen to maximize $\frac{s}{\sqrt{b}}$.

Given the response of a BDT is dependent solely on the signal and background distributions of the training variables supplied to the BDT, any systematic uncertainties that can affect these variables can potentially have an impact on the response of the BDT. This is also true for the variables used to place pre-selection cuts on the initial signal and background events before the BDT is trained, as these cuts will dictate which events the BDT is allowed to train on. The BNB flux uncertainties discussed in Section 3.1 are a source of systematic uncertainty that can affect the $\mathrm{NC} \Delta$ radiative and BNB background BDT responses. Changes in the shape of the neutrino flux distributions with respect to variables such as neutrino energy will impact the neutrino interactions, final state particles their kinematics observed in MicroBooNE. Consequently the pre-selection cuts and variables used to train the BNB based BDTs will be affected. BDTs are trained on the shape of training variable distributions and so only shape changes in the neutrino flux will impact the BNB based BDT training and responses.

The detector systematics discussed in Subsection 3.2 .4 will impact observed PMT PE, the reconstruction of particles and reconstructed particle kinematics. The amount of light produced by particles in the TPC, liquid argon purity and PMT noise will all affect observed PMT PE and therefore the light in beam-gate preselection cut and the distance from shower to closest flash training variable. Liquid argon purity and the remaining detector systematics: ionisation electron drift time, 
recombination effects, drift field uniformity, space charge effects, transverse and longitudinal diffusion of drifting electron charge, induced wire charge and wire noise all impact the observation and reconstruction of ionisation electrons into tracks and showers. As the majority of the BDT training variables are based on the position, direction and energy deposition of reconstructed tracks and showers, these uncertainties will have a significant impact on the BDT responses.

The theoretical modeling uncertainties described in Subsection 4.1.2 can also impact the BDT responses. The choice of $\mathrm{NC} \Delta$ radiative decay model and modeling of FSIs will impact the final state photon and nucleon kinematics. FSIs can also result in the production of additional particles that exit the nucleus or the reabsorption of particles into the nucleus. In terms of $\mathrm{NC} \Delta$ radiative decay, a $1 \gamma 0 p$ vertex could appear as a $1 \gamma 1 p$ vertex if an additional proton-like particle exits the nucleus. Such an instance would impact the reconstructed invariant $\Delta$ mass and thus the BDT responses as the proton kinematics would not be that of a radiatively produced proton. The opposite scenario where proton re-absorption results in a $1 \gamma 1 p$ vertex appearing as a $1 \gamma 0 p$ vertex could also occur, however the impact of this scenario is likely to be small given track information is not used in the $1 \gamma 0 p$ topology.

As for the BNB flux uncertainties, shape changes in the cross-section distributions used by GENIE to simulate neutrino interactions (resulting from changes in GENIE model parameters or choice of model) can impact neutrino interactions, final state particles and their kinematics. As the BNB background rejection BDT is trained on inclusive BNB background events, changes to cross-section parameters that alter the relative rates of different BNB interactions can change the vertex topologies the BDT will focus on at the training stage and thus impact the BDT response. Changes to the pion charge exchange probability, $x_{c e x}^{\pi}$, are an example of this. This particular parameter will affect the fraction of $\pi^{0} \mathrm{~s}$ in the BNB inclusive background, how much the BNB background rejection BDT focuses on such a background and consequently the BDT response. Changes in the GENIE branching ratio for radiative resonance decays, $x_{B R}^{R \rightarrow X+1 \gamma}$, will only impact the expected rate of $\mathrm{NC} \Delta$ radiative decay and thus will not impact the BNB based BDT responses. 


\section{Results}

This chapter contains the results of the single photon analysis using the event selection described in the previous chapter. These results include event selection efficiencies, remaining background breakdown and MicroBooNE's expected sensitivity to a single photon LEE for $6.6 \times 10^{20} \mathrm{POT}[78]$. In order to test the $\mathrm{NC} \Delta$ radiative low energy excess hypothesis, the three prediction samples (as described in Section 5.3): $\mathrm{NC} \Delta$ radiative + cosmics, $\mathrm{BNB}+$ cosmics and $\mathrm{BNB}$ external cosmic data were used. The NC $\Delta$ radiative + cosmic sample wass used as the signal prediction plus overlaid cosmic background. The BNB + cosmic sample was used as the BNB related background prediction plus overlaid cosmic background. The BNB external cosmic data sample was used as the BNB mimicking cosmic background prediction (see Subsection 5.3). Each sample was appropriately scaled to match the expected $6.6 \times 10^{20}$ POT of data. For the BNB + cosmic sample this meant the application of a flat POT scaling to the number of reconstructed vertices in the sample. The NC $\Delta$ radiative + cosmic sample was scaled by $\mathrm{POT}$ in the same way as the $\mathrm{BNB}+$ cosmic sample and then re-scaled up by a flat factor of 3 to match the predicted rate of the MiniBooNE LEE (see Chapter 2.3.1). In order to scale the BNB external cosmic data sample the number of events in the sample had to be related to POT. This was done by normalizing the number of BNB external hardware triggers in the BNB external cosmic data sample to the number of beam spills recorded in the $4.8 \times 10^{19}$ POT unblinded dataset, based on Ref [84]. Once normalized to the POT of the unblinded dataset, the BNB external cosmic data sample could be re-normalized to any POT by a flat scaling factor as was done for the other two samples.

\subsection{Event-selection Efficiency}

Tables 6.1 and 6.2 show the number of vertices, total efficiency and relative efficiency at each stage of selection for the prediction samples, separated into the $1 \gamma 0 p$ and $1 \gamma 1 p$ topologies. Note that these efficiencies are per reconstructed vertex not per generated event. The total vertices entry in each table is the number of vertices with at least one associated shower output by the vertex reconstruction algorithm (before separation by topology). Total efficiency is defined as the number of vertices at a given stage divided by the total number of vertices. Relative efficiency is defined as the number of vertices at a given stage divided by the number of vertices in the previous stage. Each table contains numbers for the for the LEE (GENIE standard- 
model rate of approximately 125 events per $6.6 \times 10^{20}$ POT $\left.\times 3\right) \mathrm{NC} \Delta$ radiative + cosmic, BNB + cosmic and BNB external cosmic data samples. The total number of reco-vertices (the second row of each table) is the number of reco-vertices output by the vertex reconstruction algorithm with exactly one associated shower. The initial selection of reco-vertices is heavily dominated by the BNB external cosmic data sample, which comprises approximately $73 \%$ of the total number of reco-vertices. Figures 6.1 and 6.2 show the signal + background stacked reconstructed shower energy prediction at each stage of the analysis after being split into the $1 \gamma 0 p$ and $1 \gamma 1 p$ topologies.

Applying pre-selection cuts reduces the BNB external cosmic data background by more than $95 \%$ for both topologies but in spite of this it remains the dominant background. Applying pre-selection cuts has a much larger impact on the $1 \gamma 1 p$ sample, which has half the total efficiency of the $1 \gamma 0 p$ sample after pre-selection. This is due to the additional track-related cuts that are placed on the $1 \gamma 1 p$ recovertices. Following pre-selection cuts, the cosmic BDT response cut is applied which almost completely removes the remaining BNB external cosmic data background for both topologies. Finally the BNB BDT response cut is applied which further reduces the remaining $\mathrm{BNB}+$ cosmic background.

\begin{tabular}{|l||c|c|c||c|c|c||c|c|c|}
\hline \multicolumn{1}{|l||}{$1 \gamma 0 p$} & \multicolumn{3}{|c||}{ LEE NC $\Delta$ Rad+Cosmic } & \multicolumn{3}{c||}{ BNB+Cosmic } & \multicolumn{3}{c|}{ BNB External Cosmic Data } \\
\hline Stage & Vertices & Tot Eff & Rel Eff & Vertices & Tot Eff & Rel Eff & Vertices & Tot Eff & Rel Eff \\
\hline Total & 599 & $100 \%$ & - & 710,000 & $100 \%$ & - & $1,900,000$ & $100 \%$ & - \\
0 track cut & 147 & $24.5 \%$ & $24.5 \%$ & 147,000 & $20.7 \%$ & $20.7 \%$ & 419,000 & $22.1 \%$ & $22.1 \%$ \\
\hline Total PE $>20$ & 132 & $22.0 \%$ & $89.8 \%$ & 97,600 & $13.7 \%$ & $66.4 \%$ & 218,000 & $11.5 \%$ & $52.0 \%$ \\
Fiducial cut & 113 & $18.9 \%$ & $85.6 \%$ & 78,900 & $11.1 \%$ & $80.8 \%$ & 156,000 & $8.2 \%$ & $71.6 \%$ \\
Reco $E_{\gamma}>30 \mathrm{MeV}$ & 91.1 & $15.2 \%$ & $80.6 \%$ & 48,900 & $6.9 \%$ & $62.0 \%$ & 85,600 & $4.5 \%$ & $54.9 \%$ \\
\hline All Precuts & 91.1 & $15.2 \%$ & - & 48,900 & $6.9 \%$ & $62.0 \%$ & 85,600 & $4.5 \%$ & - \\
Cosmic BDT & 29.6 & $4.9 \%$ & $32.5 \%$ & 952 & $0.1 \%$ & $1.9 \%$ & 313 & $0.2 \%$ & $0.4 \%$ \\
BNB BDT & 20.0 & $3.3 \%$ & $67.6 \%$ & 320 & $0.05 \%$ & $33.6 \%$ & 82.8 & $4 \mathrm{e}-3 \%$ & $26.5 \%$ \\
\hline
\end{tabular}

Table 6.1: $1 \gamma 0 p$ topology at each stage of the selection for the LEE NC $\Delta$ radiative + cosmic, BNB + cosmic and BNB external cosmic data samples. Shown are the number of vertices scaled to $6.6 \times 10^{20} \mathrm{POT}$, total efficiency and relative efficiency for each sample at each stage of selection.

Table 6.3 contains the number of generated NC $\Delta$ radiative signal events scaled by the LEE $\times 3$ enhancement factor, efficiencies and statistical significances after full selection for the $1 \gamma 0 p, 1 \gamma 1 p$ and combined topologies. The numbers shown are scaled to $6.6 \times 10^{20} \mathrm{POT}$. In spite of the rarity of NC $\Delta$ radiative decay in MicroBooNE and the substantial backgrounds, the final selection maintains a $9.3 \%$ total signal efficiency with a combined statistical significance of $1.87 \sigma$. The superior signal-background separation of the $1 \gamma 1 p$ topology in the cosmic and BNB rejection BDTs is reflected here in the statistical significances. The number of events selected for the $1 \gamma 0 p$ and $1 \gamma 1 p$ topologies is equal to the final number of selected vertices in 


\begin{tabular}{|l||c|c|c||c|c|c||c|c|c|}
\hline \multicolumn{1}{|l|}{$1 \gamma 1 p$} & \multicolumn{3}{|c||}{ LEE NC $\Delta$ Rad+Cosmic } & \multicolumn{3}{c||}{ BNB+Cosmic } & \multicolumn{3}{c|}{ BNB External Cosmic Data } \\
\hline Stage & Vertices & Tot Eff & Rel Eff & Vertices & Tot Eff & Rel Eff & Vertices & Tot Eff & Rel Eff \\
\hline Total & 599 & $100 \%$ & - & 710,000 & $100 \%$ & - & $1,900,000$ & $100 \%$ & - \\
1 track cut & 206 & $34.4 \%$ & $34.4 \%$ & 291,000 & $41.0 \%$ & $41.0 \%$ & 775,000 & $40.8 \%$ & $40.8 \%$ \\
\hline Total PE $>20$ & 181 & $30.2 \%$ & $87.8 \%$ & 191,000 & $41.0 \%$ & $65.6 \%$ & 380,000 & $20.0 \%$ & $49.0 \%$ \\
Fiducial cut & 141 & $23.6 \%$ & $77.9 \%$ & 140,000 & $19.7 \%$ & $73.2 \%$ & 244,000 & $12.8 \%$ & $64.2 \%$ \\
Track $<100 \mathrm{~cm}$ & 108 & $18.1 \%$ & $76.6 \%$ & 87,200 & $12.3 \%$ & $62.3 \%$ & 152,000 & $8.0 \%$ & $62.3 \%$ \\
Reco E $E_{\gamma}>30 \mathrm{MeV}$ & 70.6 & $11.8 \%$ & $65.3 \%$ & 37500 & $5.3 \%$ & $42.9 \%$ & 52,500 & $2.8 \%$ & $34.5 \%$ \\
Shower gap $>1 \mathrm{~cm}$ & 57.8 & $9.7 \%$ & $81.9 \%$ & 25,500 & $3.6 \%$ & $68.1 \%$ & 37,700 & $2.0 \%$ & $71.8 \%$ \\
Good calo cut & 50.2 & $8.4 \%$ & $86.9 \%$ & 20,600 & $2.9 \%$ & $80.6 \%$ & 28,600 & $1.5 \%$ & $75.9 \%$ \\
Flipped track cut & 40.4 & $6.7 \%$ & $80.4 \%$ & 12,800 & $1.8 \%$ & $62.5 \%$ & 16,400 & $0.9 \%$ & $57.3 \%$ \\
Back-to-back cut & 37.3 & $6.2 \%$ & $92.4 \%$ & 11,200 & $1.6 \%$ & $87.5 \%$ & 13,800 & $0.7 \%$ & $84.1 \%$ \\
\hline All Precuts & 37.3 & $6.2 \%$ & - & 11,200 & $1.6 \%$ & - & 13,800 & $0.7 \%$ & - \\
Cosmic BDT & 26.1 & $4.4 \%$ & $70.0 \%$ & 830 & $0.1 \%$ & $7.4 \%$ & 49.1 & $3 \mathrm{e}-3 \%$ & $0.4 \%$ \\
BNB BDT & 14.6 & $2.4 \%$ & $55.9 \%$ & 82.1 & $0.01 \%$ & $9.9 \%$ & 3.1 & $2 \mathrm{e}-4 \%$ & $6.3 \%$ \\
\hline
\end{tabular}

Table 6.2: As for Table 6.1 but for the $1 \gamma 1 p$ topology.
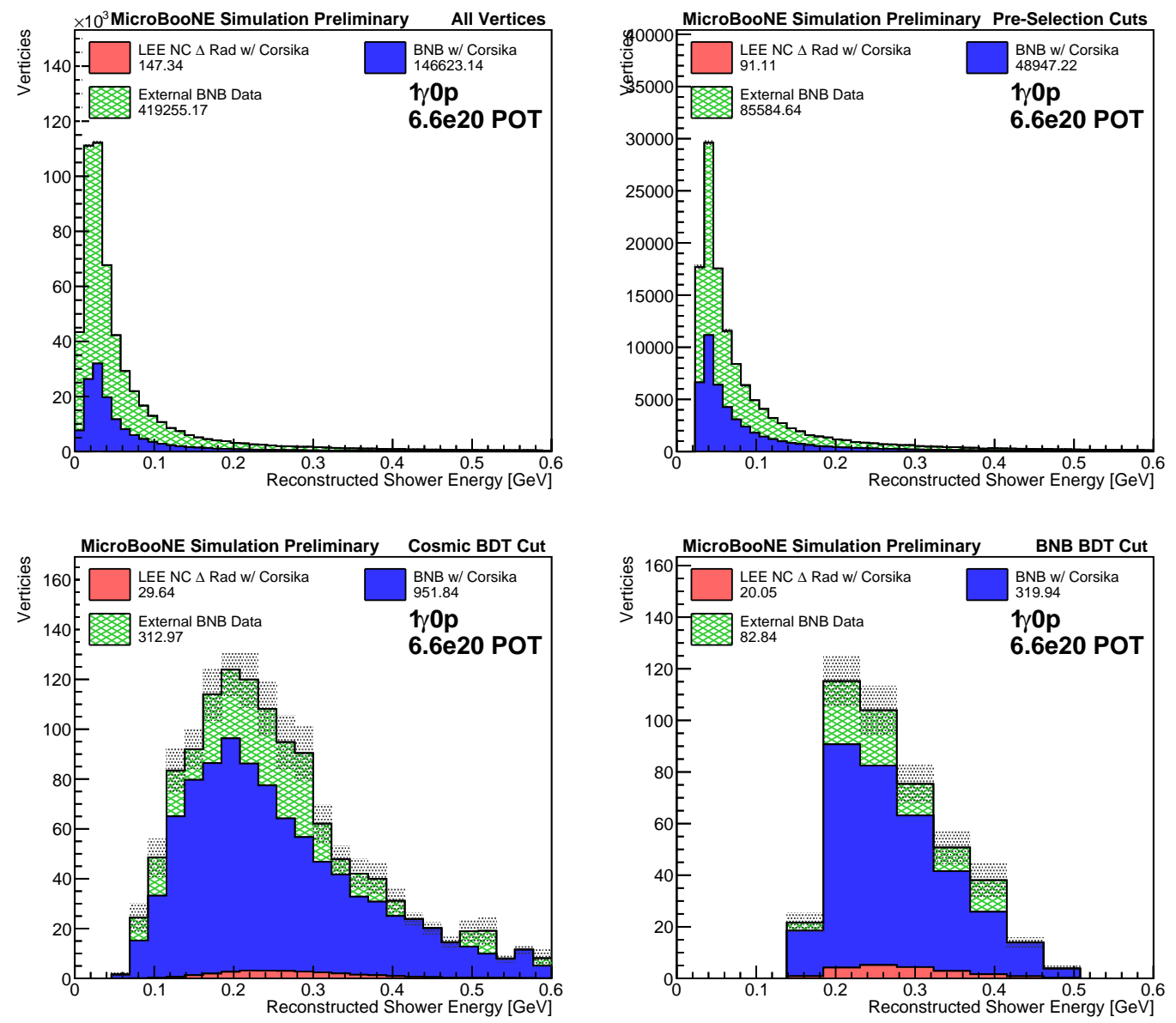

Figure 6.1: $1 \gamma 0 p$ reconstructed shower energy prediction using the stacked LEE scaled NC $\Delta$ radiative + cosmic, $\mathrm{BNB}+$ cosmic and BNB external cosmic data samples normalized to $6.6 \times 10^{20}$ POT at each stage of selection. Top left: total one-shower vertices, top right: pre-selection cuts applied, bottom left: cosmic BDT applied, bottom right: BNB BDT applied. The gray bars show the statistical uncertainty of each bin. 

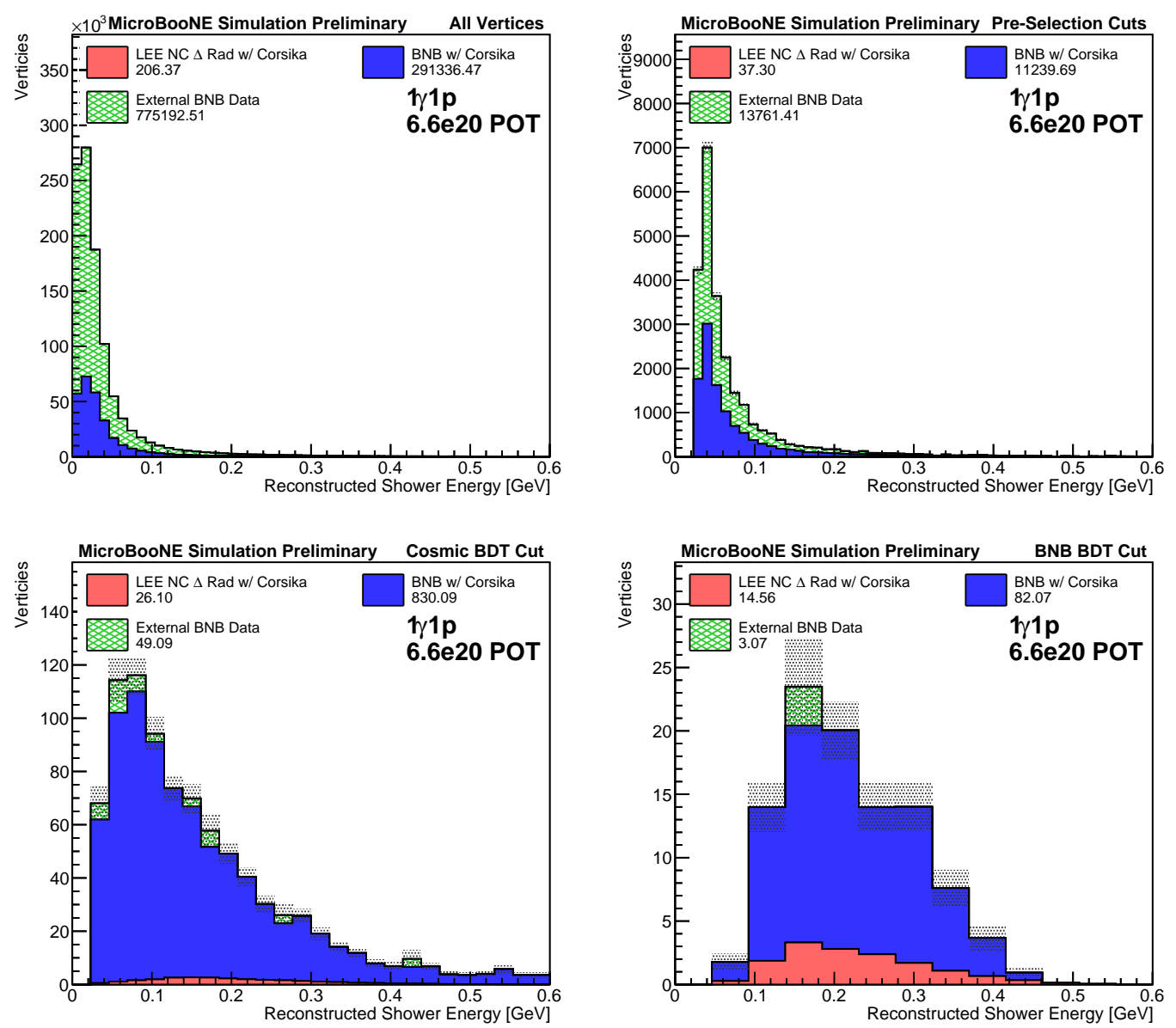

Figure 6.2: As for Figure 6.1 but for the $1 \gamma 1 p$ topology.

Tables 6.1 and 6.2 respectively, as by the end of selection only one vertex remains per event. This is also true for the remaining backgrounds. In the instance of multiple selected vertices per event, the vertex with the highest BNB response value would be chosen.

\begin{tabular}{|l|c|c|c|c|}
\hline & Generated Events & Selected Events & Efficiency \% & Significance $\left(\frac{s}{\sqrt{b}}\right)$ \\
\hline $1 \gamma 0 p$ & 213 & 20.0 & 9.4 & $1.00 \sigma$ \\
$1 \gamma 1 p$ & 159 & 14.6 & 9.2 & $1.58 \sigma$ \\
Combined & 372 & 34.6 & 9.3 & $1.87 \sigma$ \\
\hline
\end{tabular}

Table 6.3: LEE NC $\Delta$ radiative signal events (in-TPC), number of events after full selection, full selection efficiency and statistical significance for the $1 \gamma 0 p, 1 \gamma 1 p$ and combined topologies. Numbers include the LEE $\times 3$ factor and are scaled to $6.6 \times 10^{20}$ POT. Combined statistical significance is calculated by adding-in-quadrature the $1 \gamma 0 p$ and $1 \gamma 1 p$ statistical significances.

As was mentioned in Subsection 5.6.1. use of the reduced-correlations variable list resulted in marginally worse signal-background separation in all BDT responses 
compared to using the full variable list. This marginal difference results in a noticeable loss in statistical significance for the LEE NC $\Delta$ radiative signal. Table 6.4 displays the same information as Table 6.3 but for the selection using the BDTs trained with the reduced-correlations variable list. The $1 \gamma 0 p, 1 \gamma 1 p$ and combined topologies all show lower statistical significance after the full selection has been applied compared to Table 6.3, motivating the use of the full variable list for BDT training.

\begin{tabular}{|l|c|c|c|c|}
\hline & Generated Events & Selected Events & Efficiency $\%$ & Significance $\left(\frac{s}{\sqrt{b}}\right)$ \\
\hline $1 \gamma 0 p$ & 213 & 20.2 & 9.5 & $0.9 \sigma$ \\
$1 \gamma 1 p$ & 159 & 11.8 & 7.4 & $1.16 \sigma$ \\
Combined & 372 & 32.0 & 9.3 & $1.47 \sigma$ \\
\hline
\end{tabular}

Table 6.4: As for Table 6.3, but using the BDTs trained with the reducedcorrelations variable list described in Subsection 5.6.1.

\subsection{Background Composition}

Figures 6.3 and 6.4 show the true composition of the BNB + cosmic background prediction for the $1 \gamma 1 p$ and $1 \gamma 0 p$ topologies at each stage of event selection. Initially cosmic activity dominates the background but it is drastically reduced as the various event selection stages (particularly the pre-selection cuts and the cosmic BDT) are applied. This is the cosmic activity simulated by CORSIKA that is present in the TPC at the same time as BNB background neutrino interactions. The BNB mimicking cosmic background predicted by the BNB external cosmic data sample is not included. For both topologies the most significant remaining background post-selection is NC $\pi^{0} \rightarrow 2 \gamma$ decay, where one of the two photons is not observed. This results in a single photon shower in the TPC that looks kinematically very similar to a single photon from $\mathrm{NC} \Delta$ radiative decay. The $1 \gamma 0 p$ topology also has moderate contributions from $\mathrm{CC} \pi^{0} \rightarrow 2 \gamma$ decay where the muon track has been not been correctly reconstructed, making these vertices look identical to their NC counterparts.

There are multiple ways that one of the two photons in $\pi^{0} \rightarrow 2 \gamma$ decay can be missed by the event-selection:

- Escaping photon: a $\pi^{0}$ decays into two photons inside the TPC. One photon produces a shower inside the TPC while the other exits. This background could potentially be reduced with a more aggressive fiducial cut but the position of 

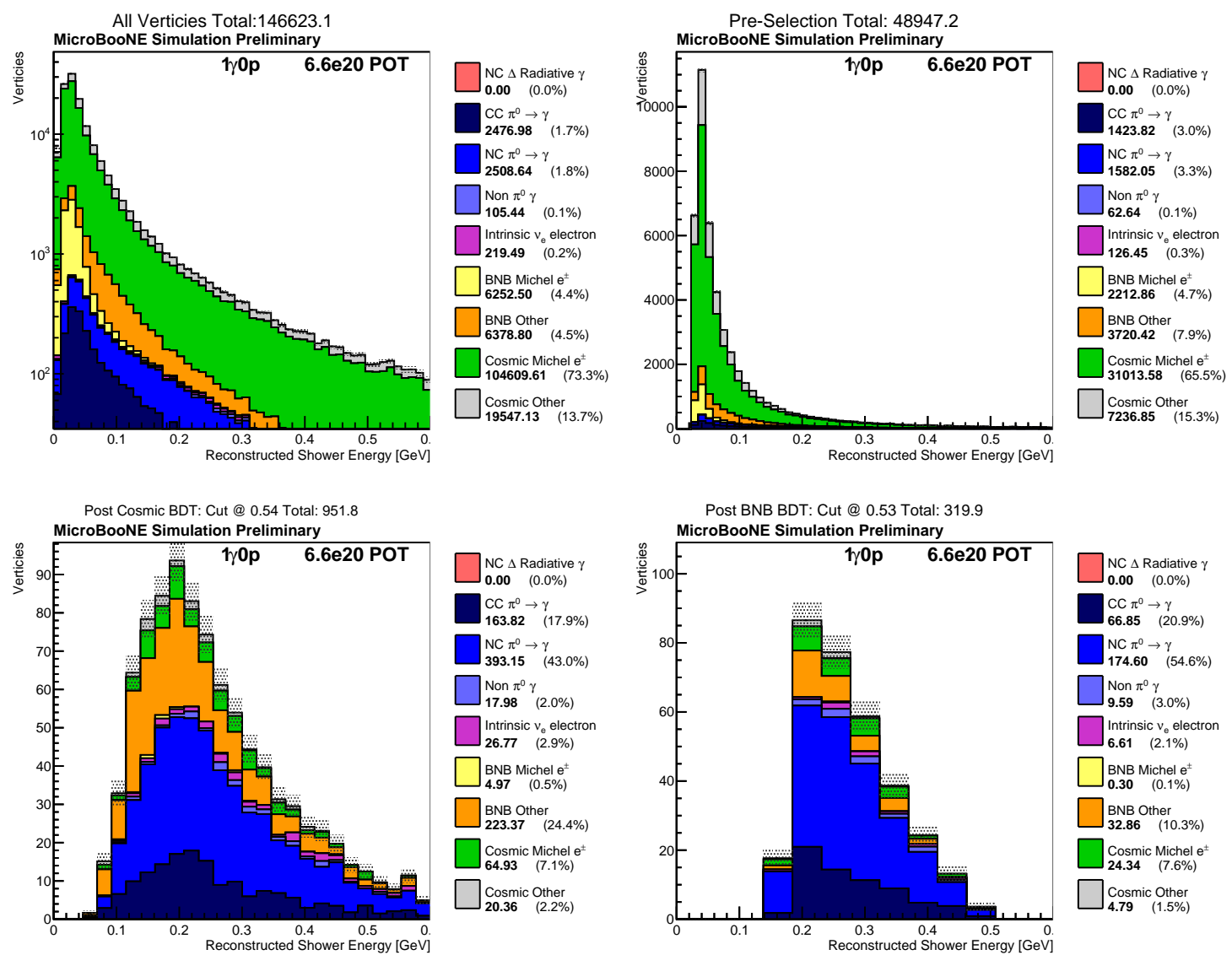

Figure 6.3: Predicted $1 \gamma 0 p$ BNB + cosmic reconstructed shower energy distribution for each stage of the analysis, broken down by particle and interaction type. Top left: vertices output by the vertex reconstruction algorithm (log scale y-axis), top right vertices remaining after pre-selection cuts, bottom left: vertices remaining after the cosmic BDT cut, bottom right: vertices remaining after the BNB BDT cut.

the reco-vertex in the TPC is already one of the variables used to train the background rejection BDTs so it would likely have little effect.

- Entering photon: opposite to the previous case, a $\pi^{0}$ decays into two photons in the argon outside the TPC. One photon enters and produces a shower in the TPC while the second photon does not enter the TPC. Could be reduced by a more aggressive fiducial cut.

- Asymmetric decay: one of the photons produced takes the majority of the energy of the decay and is properly reconstructed. The second photon has very little energy and is reconstructed poorly.

- Tagged as cosmic: one of the two photons is erroneously tagged as cosmic activity.

- Unresponsive wire region: one of the two photons deposits its energy 

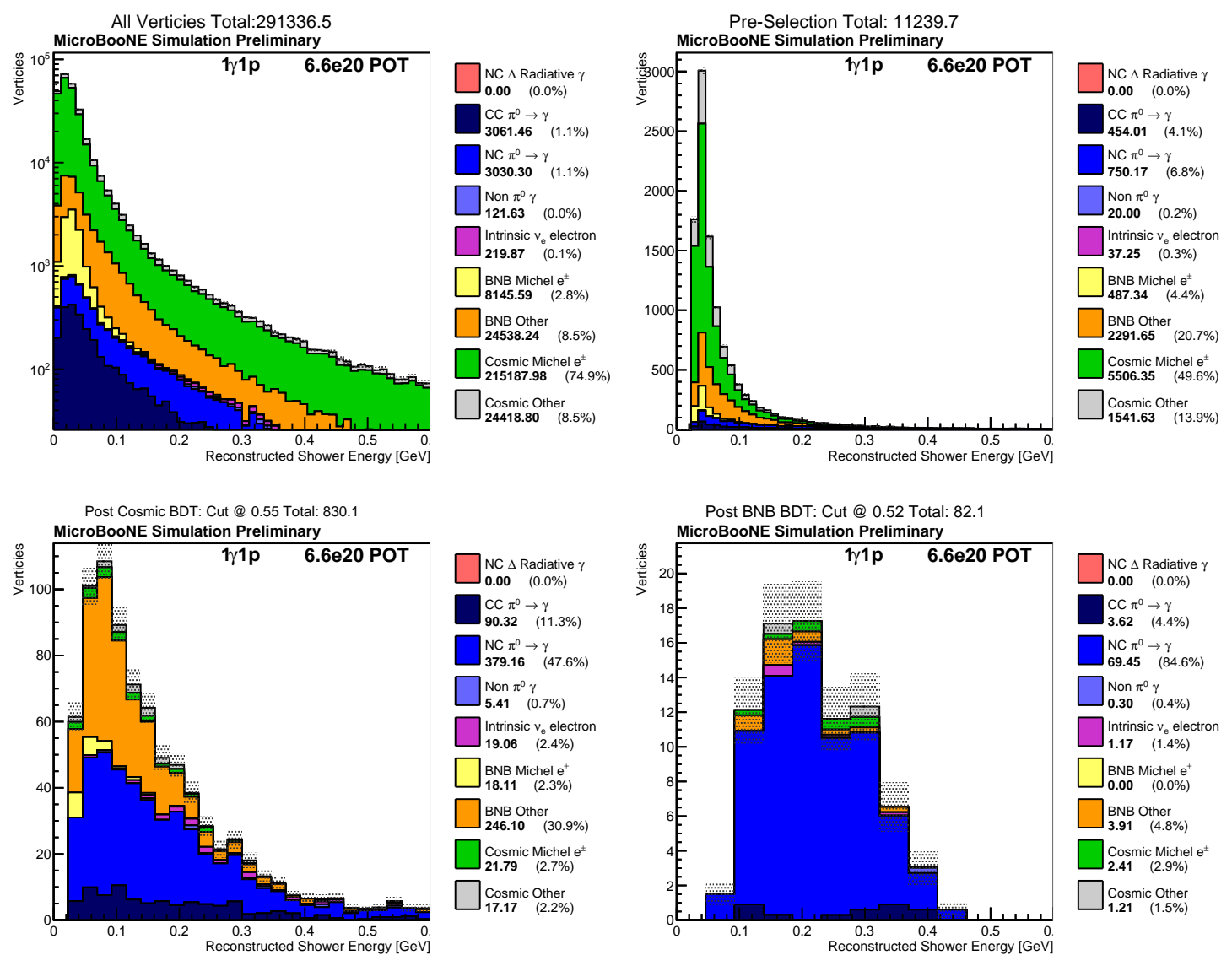

Figure 6.4: As for 6.4 but for the $1 \gamma 1 p$ topology.

in a region of the TPC where the wires are unresponsive, resulting in the reconstruction of only one shower.

- Shower merging: the two photons are mostly forward facing with a small opening angle and are mis-reconstructed into a single shower or one of the showers overlaps with a track resulting in a merged track or shower.

- Reconstructed as a track: one of the photons is a relatively straight shower with few branches and is mis-reconstructed as a track.

- Vertex reconstruction failure: the vertex reconstruction algorithm fails to correctly associated two correctly reconstructed showers together, potentially resulting in two signal vertex candidates in the TPC.

Table 6.5 shows the contributions made by the categories above to the total $1 \gamma 1 p$ $\mathrm{NC} \pi^{0}$ background. As the most powerful differentiator for the NC $\pi^{0}$ background is the second shower, looking for additional photon-like activity that was missed by reconstruction could yield additonal background reduction. A deep learning approach such as a semantic segmentation network (SSN) could be trained to identify 
the activity of the missed second shower and allow for significant additional $\mathrm{NC} \pi^{0}$ background reduction. SSNs have been effectively applied in MicroBooNE analyses before [85] and this approach is currently being studied for the single photon analysis. Additional background reduction by identification of the missing second shower activity is applicable to two of the categories listed in Table 6.5. "asymmetric decay" and "reconstructed as a track". Combined these two categories represent $53 \%$ of the total NC $\pi^{0}$ background, thus reduction in these categories could have a significant impact on the sensitivity of the analysis.

\begin{tabular}{|l|c|}
\hline Reason for only one shower & \% of $\pi^{0}$ background \\
\hline Asymmetric decay & 40.1 \\
Tagged as cosmic & 10.4 \\
Unresponsive wire region & 8.6 \\
Merged showers & 15.1 \\
Reconstructed as a track & 12.9 \\
Other & 12.8 \\
\hline
\end{tabular}

Table 6.5: Contributions to the $1 \gamma 1 p$ NC $\pi^{0}$ background.

A potential source of $\pi^{0} \rightarrow 2 \gamma$ not considered in this analysis are "dirt events". These are events in which BNB neutrinos interact with earth and other material surrounding the detector. Photons produced by neutrino $\pi^{0} \rightarrow 2 \gamma$ decay can subsequently find their way into the MicroBooNE TPC and so are a potential background to the analysis. Dirt events were considered in MiniBooNE and were an irreducible background to the LEE. The most recent LEE results released by the MiniBooNE collaboration predict 75.2 dirt events per $12.48 \times 10^{20}$ POT [23]. A simple scaling from MiniBooNE to MicroBooNE can be performed to estimate the number of expected photon dirt events in the MicroBooNE TPC:

$$
\frac{N_{\mu \mathrm{B}}}{N_{\mathrm{MB}}}=\frac{V_{\mu \mathrm{B}}}{V_{\mathrm{MB}}} \frac{\phi_{\mu \mathrm{B}}}{\phi_{\mathrm{MB}}} \frac{P_{\mu \mathrm{B}}}{P_{\mathrm{MB}}} \frac{\epsilon_{\mu \mathrm{B}}}{\epsilon_{\mathrm{MB}}},
$$

where $\mu \mathrm{B}$ and $\mathrm{MB}$ refer to MicroBooNE and MiniBooNE respectively, $N$ is the number of expected dirt events, $V$ is the volume of the detector where particles are visible (i.e. the MicroBooNE TPC), $\phi$ is the BNB neutrino flux at the detector, $P$ is the expected POT and $\epsilon$ is the fraction of remaining dirt events post-selection.

$V_{\mu \mathrm{B}}$ is calculated using the dimensions of the TPC: $2.33 \times 2.56 \times 10.37 \mathrm{~m}^{3} 43$ and $V_{\mathrm{MB}}$ is calculated using the radius of the inner-sphere of MiniBooNE: $\frac{4}{3} \pi \times 6.1^{3}$ $\mathrm{m}^{3}[86]$. Both $\phi_{\mu \mathrm{B}}$ and $\phi_{\mathrm{MB}}$ are proportional to $\frac{1}{r^{2}}$, where $r$ is the distance between the beam target and the detector, due to the divergence of the BNB [53]. Hence $\phi_{\mu \mathrm{B}}=\frac{1}{470^{2}} \mathrm{~m}^{-2}$ and $\phi_{\mathrm{MB}}=\frac{1}{541^{2}} \mathrm{~m}^{-2}$. For this analysis $P_{\mu \mathrm{B}}=6.6 \times 10^{20} \mathrm{POT}$ while for the latest MiniBooNE LEE result $P_{\mathrm{MB}}=12.48 \times 10^{20}$ POT. Finally $\epsilon_{\mu \mathrm{B}}$ 
is assumed to be equal to the fraction of remaining $1 \gamma 0 p \mathrm{NC} \pi^{0}$ background postselection (in reality the inward facing direction of the dirt showers would likely lower this value) which is equivalent to $7 \%$ and $\epsilon_{\mathrm{MB}}=17 \%$ [87]. Using these values and the MiniBooNE predicted 75.2 dirt events per $12.48 \times 10^{20}$ POT, the expected dirt background for this analysis is approximately 1.4 events per $6.6 \times 10^{20}$ POT, making it an insignificant background compared to the dominant BNB NC $\pi^{0}$ background.
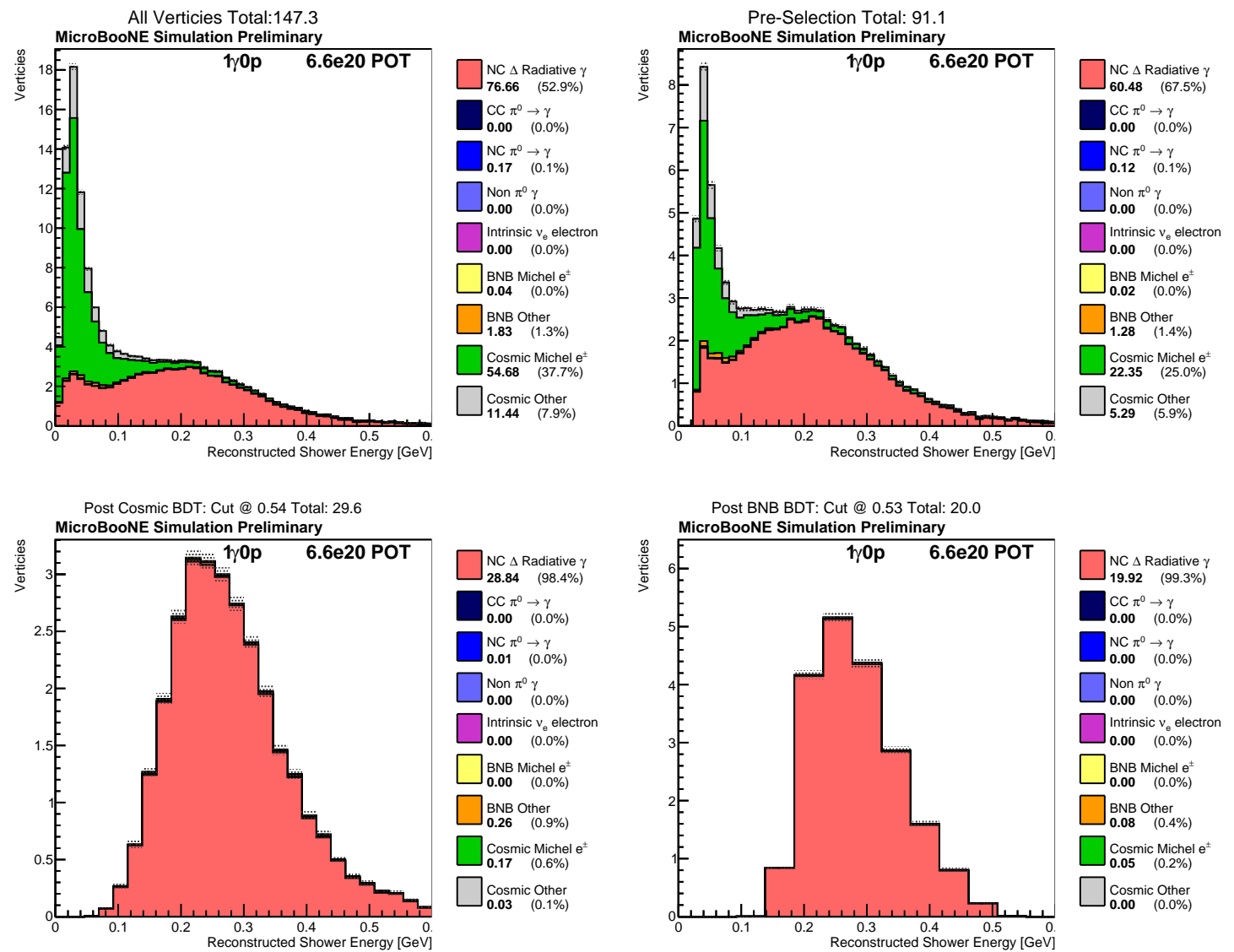

Figure 6.5: Predicted $1 \gamma 0 p$ NC $\Delta$ radiative + cosmic reconstructed shower energy distribution for each stage of the analysis, broken down by particle and interaction type. Top left: vertices output by the vertex reconstruction algorithm, top right vertices remaining after pre-selection cuts, bottom left: vertices remaining after cosmic BDT cut, bottom right: vertices remaining after BNB BDT cut.

Figures 6.5 and 6.6 shows the breakdown of the $\mathrm{NC} \Delta$ radiative + cosmic prediction sample for the $1 \gamma 0 p$ and $1 \gamma 1 p$ topologies through each stage of selection. Top left: vertices output by the vertex reconstruction algorithm, top right vertices remaining after pre-selection cuts, bottom left: vertices remaining after cosmic BDT cut, bottom right: vertices remaining after BNB BDT cut. As for the BNB + cosmic breakdown, only the cosmic activity simulated by CORSIKA that is present in the TPC at the same time as the BNB neutrino induced NC $\Delta$ radiative events is 

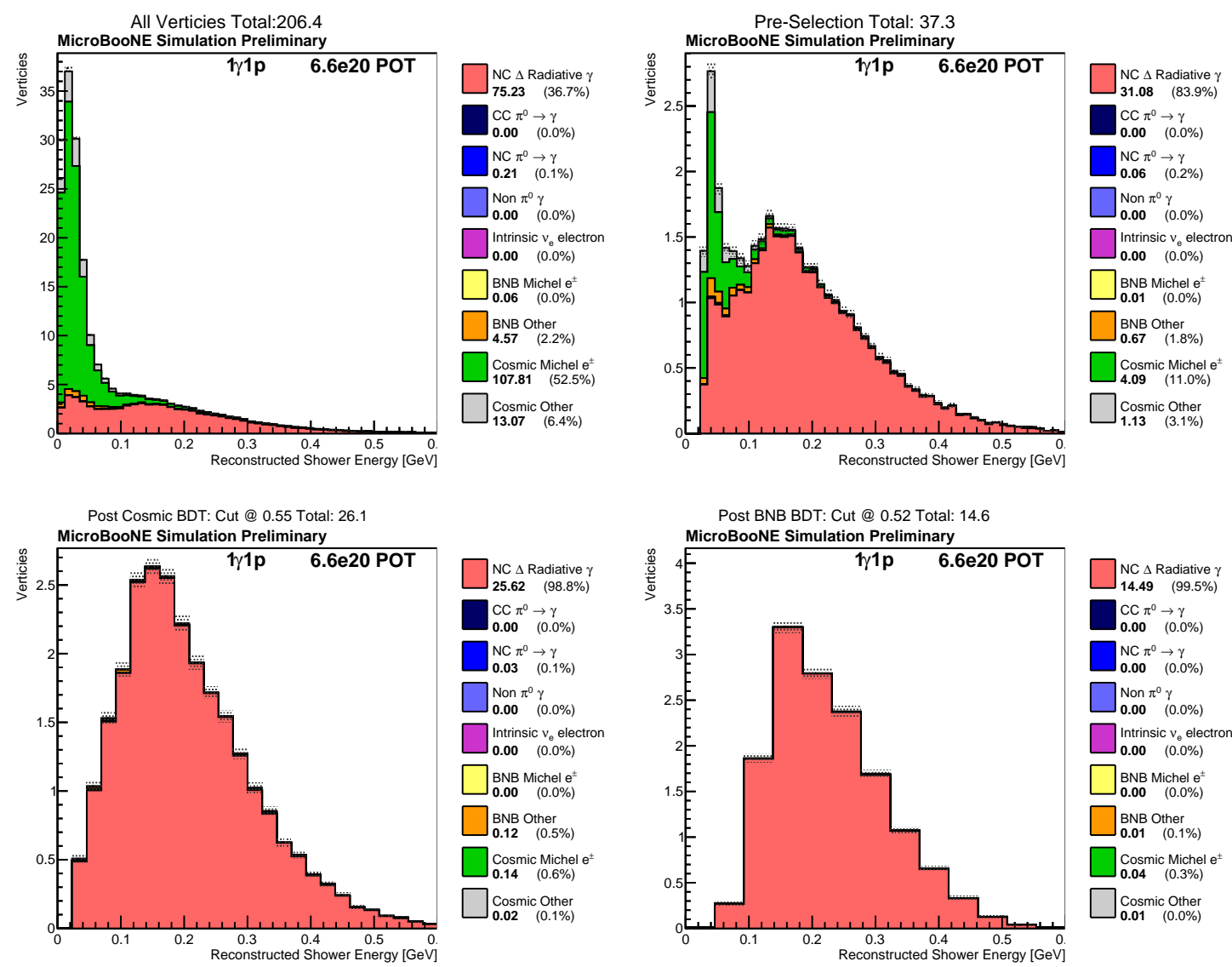

Figure 6.6: As for 6.5 but for the $1 \gamma 1 p$ topology.

included. Once again cosmics initially dominate, before being substantially reduced by the event selection. Post selection the remaining vertices from the NC $\Delta$ radiative + cosmic sample are almost entirely $\mathrm{NC} \Delta$ radiative signal vertices. There is no real improvement between the cosmic BDT cut and the BNB BDT cut for this sample, however application of the BNB BDT cut is important in reducing BNB related backgrounds as can be seen in the bottom plots of Figures 6.3 and 6.4 .

\subsection{Invariant $\Delta$ Mass}

One variable of particular interest to the analysis that is used to train the $1 \gamma 1 p$ cosmic and BNB rejection BDTs is the reconstructed invariant $\Delta$ mass $\left(W_{\Delta}\right)$. $W_{\Delta}$ is calculated under the assumption that a $\Delta^{+} \rightarrow p+\gamma$ radiative decay occurred, the $p$ and $\gamma$ did not undergo any further interactions before exiting the nucleus and no other particles were produced in FSIs that exited the argon nucleus, using

$$
W_{\Delta}^{2}=m_{p}^{2}+2 E_{\gamma}\left(E_{p}-\left|\vec{p}_{p}\right| \cos \theta_{p \gamma}\right)
$$


where $m_{p}$ is the mass of a proton, $E_{\gamma}$ is the energy of the photon, $E_{p}$ is the energy of the proton, $\left|\vec{p}_{p}\right|$ is the magnitude of momentum of the proton and $\theta_{p \gamma}$ is the opening angle between the proton and photon. The non-visibility of neutrons in MicroBooNE precludes the calculation of a $\Delta^{0} \rightarrow n+\gamma$ invariant mass. For this reason the reconstructed $W_{\Delta}^{2}$ is calculated for the $1 \gamma 1 p$ topology vertices only, using the associated reconstructed shower energy as $E_{\gamma}$, associated reconstructed track energy $+m_{p}$ as $E_{p}, \sqrt{E_{p}^{2}-m_{p}^{2}}$ as $\left|\vec{p}_{p}\right|$ and the opening angle between the initial directions of the track and shower as $\theta_{p \gamma}$.

\section{Pre-Selection Cuts}

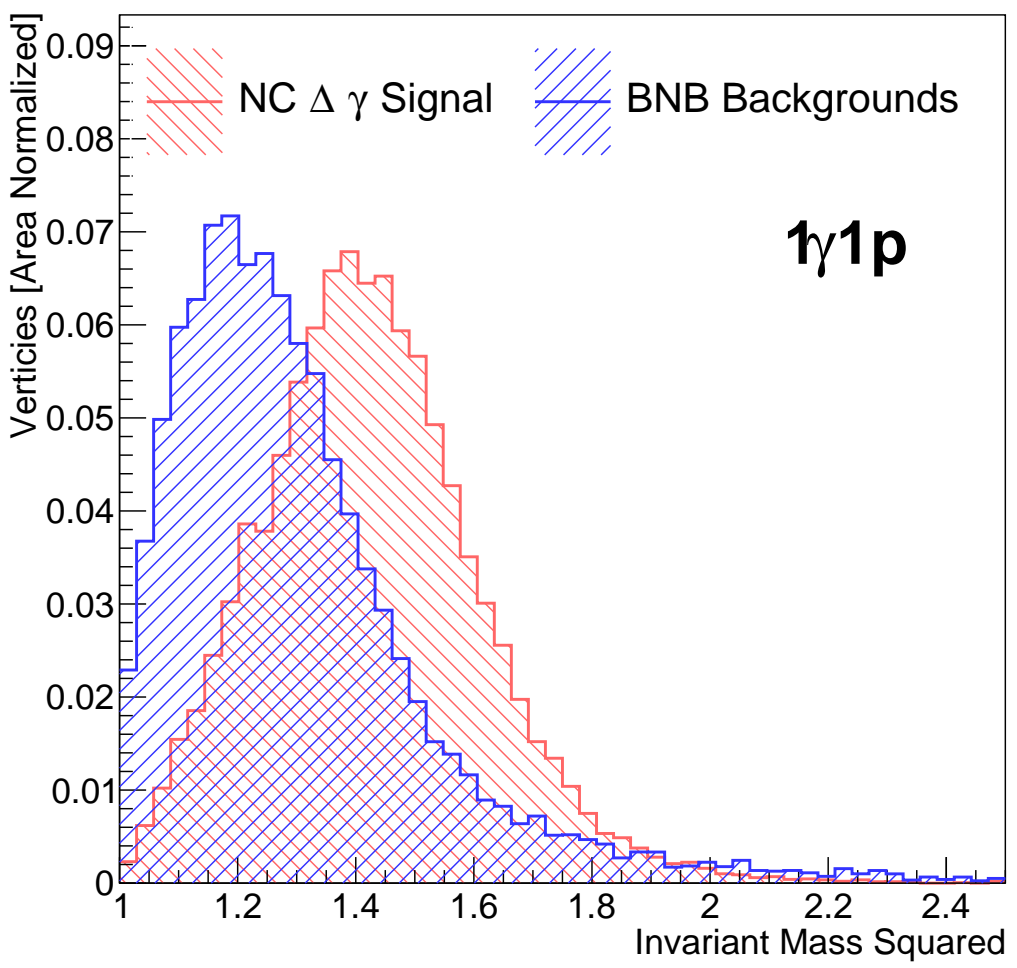

Figure 6.7: Reconstructed invariant mass squared for the $\mathrm{NC} \Delta$ radiative sample (red) and BNB sample (blue) used to train the $1 \gamma 1 p$ BNB rejection BDT.

Figure 6.7 shows the reconstructed $W_{\Delta}^{2}$ distributions for the NC $\Delta$ radiative and $\mathrm{BNB}$ vertices used to train the $1 \gamma 1 p$ BNB rejection BDT. The $W_{\Delta}^{2}$ distribution for the NC $\Delta$ radiative sample peaks at approximately $1.4 \mathrm{GeV}^{2}$ which falls slightly short of the true $W_{\Delta}^{2}=1.232^{2}=1.518 \mathrm{GeV}^{2}$ value. This is due to typically lower reconstructed track and shower energy values compared to the true energy of the incident particles that produced them. Attempts to mitigate factors that contribute to this effect such as fewer electrons reconstructed on the TPC wires than were on the wires in truth and recombination have been made at the energy reconstruction 


\section{NC $\Delta$ Radiative Photon Showers}

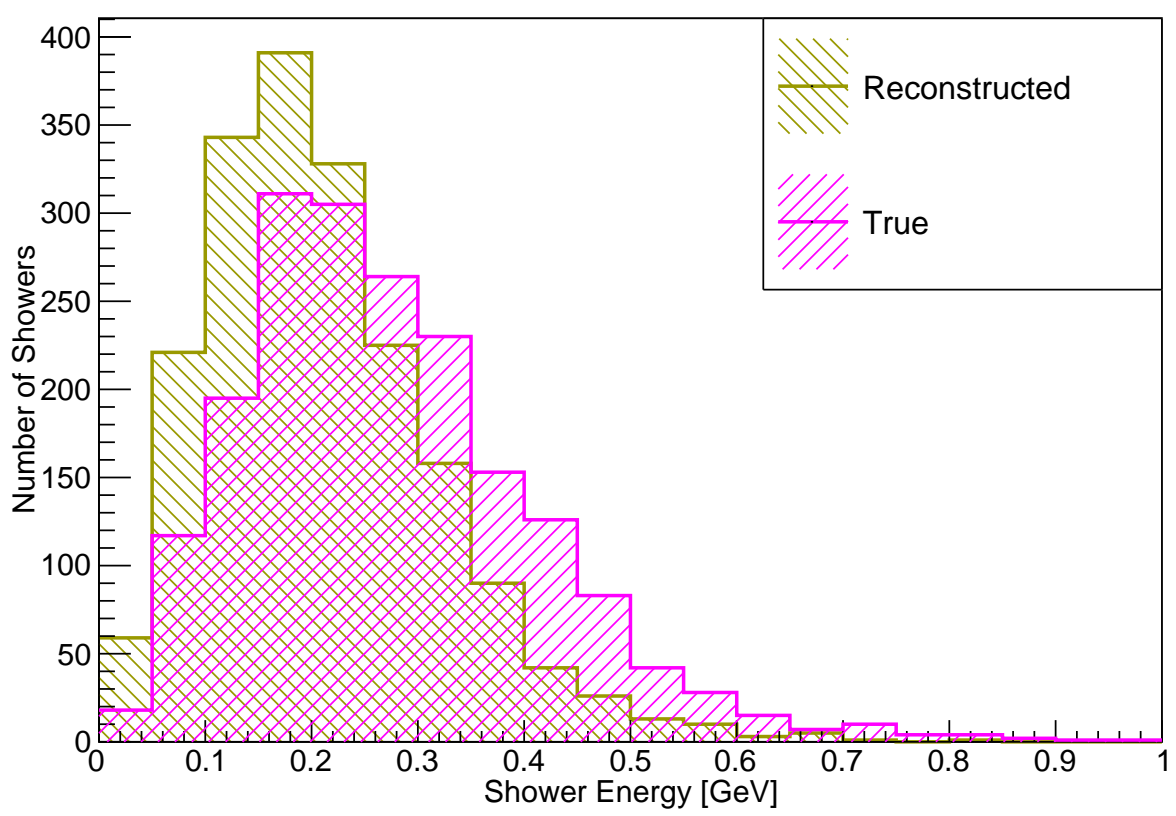

Figure 6.8: Reconstructed and associated true NC $\Delta$ radiative photon shower energies after pre-selection cuts.

stage (see Chapter 4), however an energy deficit remains as can be seen for showers in Figure 6.8 .

Figure 6.9 shows the signal + background stacked $W^{2}$ prediction at each stage of event selection. Initially the background distributions differ greatly to the expected $W_{\Delta}^{2}$ distribution, however by the final stage of selection only background vertices kinematically similar to signal vertices remain. This results in a reconstructed background $W_{\Delta}^{2}$ distribution very similar to the signal $W_{\Delta}^{2}$ distribution. Further compounding this similarity is the fact that the majority of the remaining background post-selection consists of a single shower produced by $\pi^{0} \rightarrow 2 \gamma$ decay plus a track. Given the most common decay mode for a $\Delta$ resonance is $\Delta \rightarrow N+\pi$, if this decay happens to be $\Delta^{+} \rightarrow p+\pi^{0}$ where the pion decay is highly asymmetric or the two photons from the decay are mis-reconstructed into a single shower, the resulting reconstructed vertex will be have a single associated shower and track both of whose kinematics will greatly resemble the $\Delta^{+} \rightarrow p+\gamma$ shower and track kinematics. This highlights the reason for the strong similarity between signal and remaining $\pi^{0}$ background in terms of the reconstructed $W_{\Delta}^{2}$. 

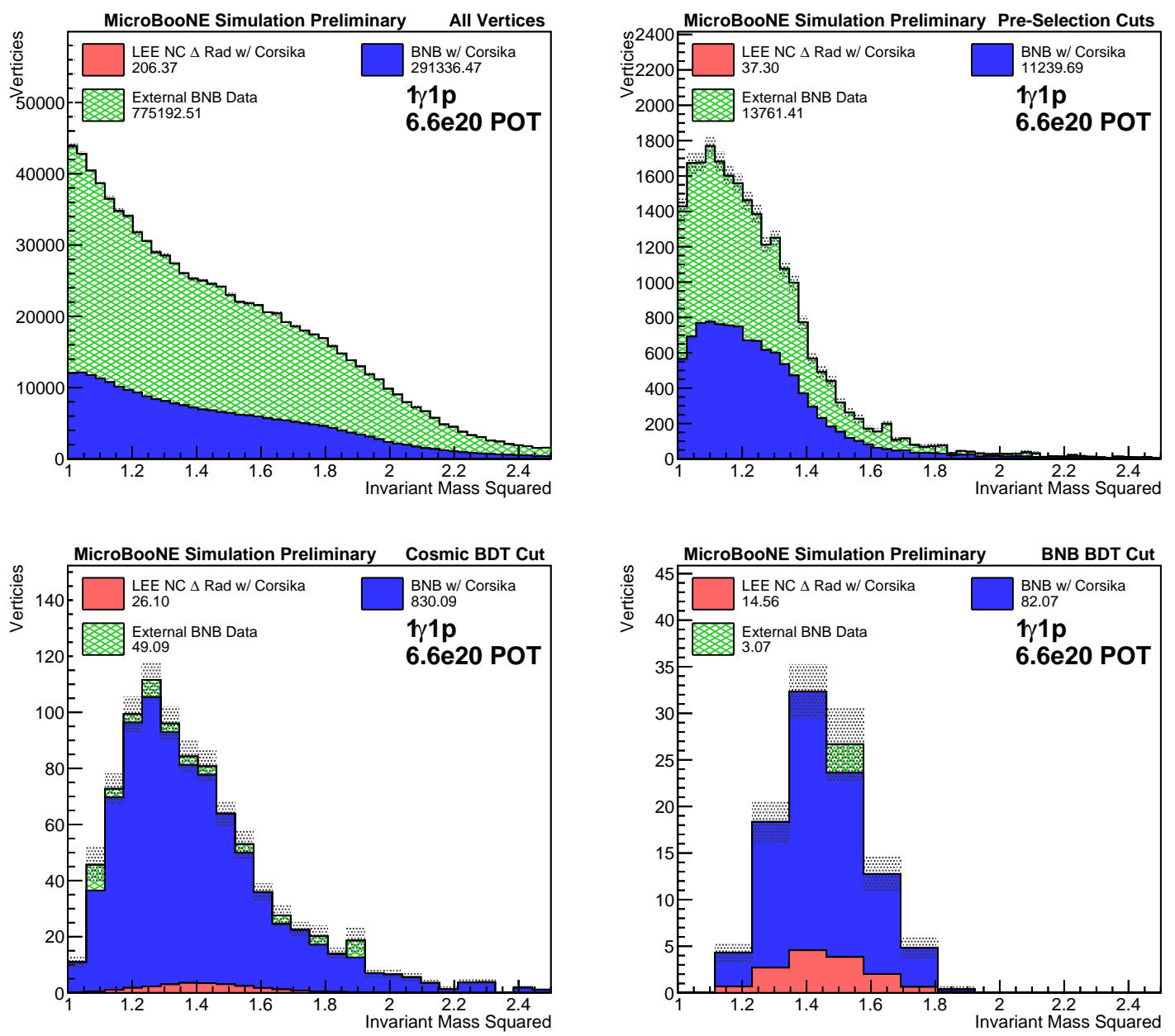

Figure 6.9: $1 \gamma 1 p$ reconstructed $W_{\Delta}^{2}$ for the stacked LEE NC $\Delta$ radiative + cosmic, $\mathrm{BNB}+$ cosmic and BNB external cosmic data samples normalized to $6.6 \times 10^{20}$ POT at each stage of selection. Top left: total one-shower vertices, top right: preselection cuts applied, bottom left: cosmic BDT applied, bottom right: BNB BDT applied. The gray bars show the statistical uncertainty of each bin.

\subsection{Data-Monte Carlo Comparison}

In order to validate the event selection a data-MC comparison was performed using the three prediction samples (as described in Section 5.3) and the MicroBooNE unblinded data sample:

- NC $\Delta$ radiative + cosmic: $\mathrm{MC}$ predictions for $\mathrm{NC} \Delta$ radiative signal events from GENIE and CORSIKA simulated cosmics overlaid in the same signal events.

- BNB + cosmic: MC predictions for BNB background events from GENIE and CORSIKA simulated cosmics overlaid in the same BNB background events. 
- BNB external cosmic data: Data prediction for BNB mimicking cosmic background (recorded BNB events that have no BNB neutrino interaction, only cosmics, see Section 5.3) from MicroBooNE data taken outside the BNB beam-spill window.

- Unblinded data: $4.8 \times 10^{19}$ POT of unblinded MicroBooNE data.

The three prediction samples were scaled to $4.8 \times 10^{19}$ POT (in the manner described at the beginning of the chapter) and stacked together to be compared against the unblinded data sample. As the BNB external cosmic data sample is not a MC sample, the comparison between the stacked prediction samples and the unblinded data sample is not a "true" data-MC comparison. Henceforth the stacked prediction samples will be referred to as $\mathrm{MC}+\mathrm{EXT}$ (MC corresponding to $\mathrm{NC} \Delta$ radiative + cosmic and BNB + cosmic, EXT corresponding to BNB external cosmic data) and the unblinded data sample will be referred to simply as data. Figures 6.10 to 6.14 show the data-MC+EXT comparisons for all $1 \gamma 1 p$ BDT training variables after preselection cuts have been applied. Good shape agreement is seen between MC+EXT and data for the variables shown. There are minor normalization differences of $1 \%$ for the $1 \gamma 1 p$ sample and $4 \%$ for the $1 \gamma 0 p$ sample. This indicates that the MC+EXT prediction well represents the $4.8 \times 10^{19}$ POT unblinded MicroBooNE data. 

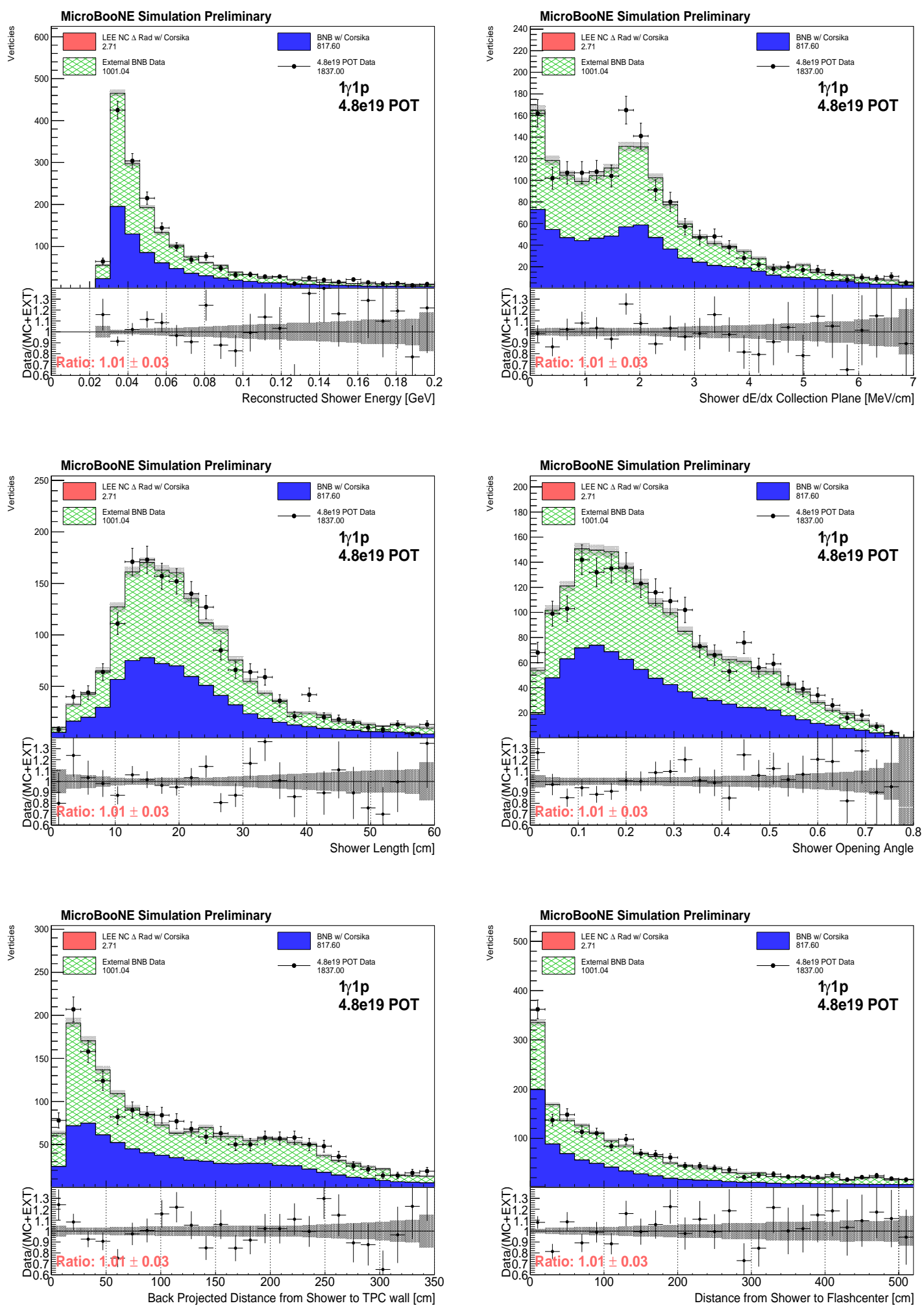

Figure 6.10: $1 \gamma 1 p$ data-MC+EXT comparison for several variables directly after pre-selection. The top panel contains the variable with the data superimposed on the $\mathrm{MC}+\mathrm{EXT}$ samples. The bottom panel contains the ratio of data to $\mathrm{MC}+\mathrm{EXT}$ per bin. Statistical data errors are included on the ratio points, statistical MC+EXT errors are shown in the gray bands. From top left to bottom right: shower energy, shower $\mathrm{dE} / \mathrm{dx}$, shower length, shower opening angle, backwards-projected shower distance from TPC wall and distance between shower and closest flash ( $z$-axis). 

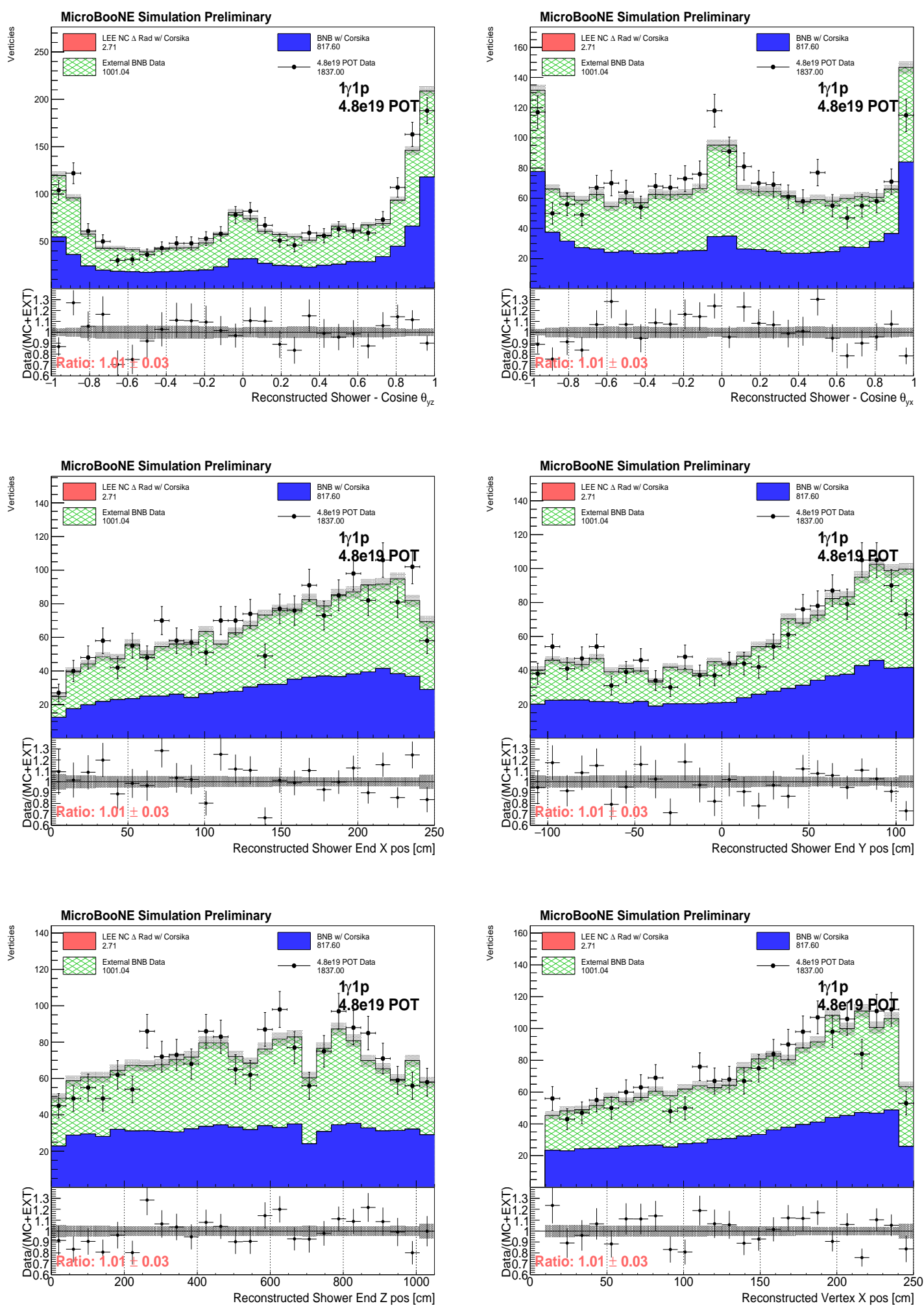

Figure 6.11: $1 \gamma 1 p$ data-MC+EXT comparison for several variables directly after pre-selection. The top panel contains the variable with the data superimposed on the $\mathrm{MC}+\mathrm{EXT}$ samples. The bottom panel contains the ratio of data to $\mathrm{MC}+\mathrm{EXT}$ per bin. Statistical data errors are included on the ratio points, statistical MC+EXT errors are shown in the gray bands. From top left to bottom right: shower $\cos \theta_{y z}$, shower $\cos \theta_{y x}$, shower end $x$-position, shower end $y$-position, shower end $z$-position and vertex $x$-position. 

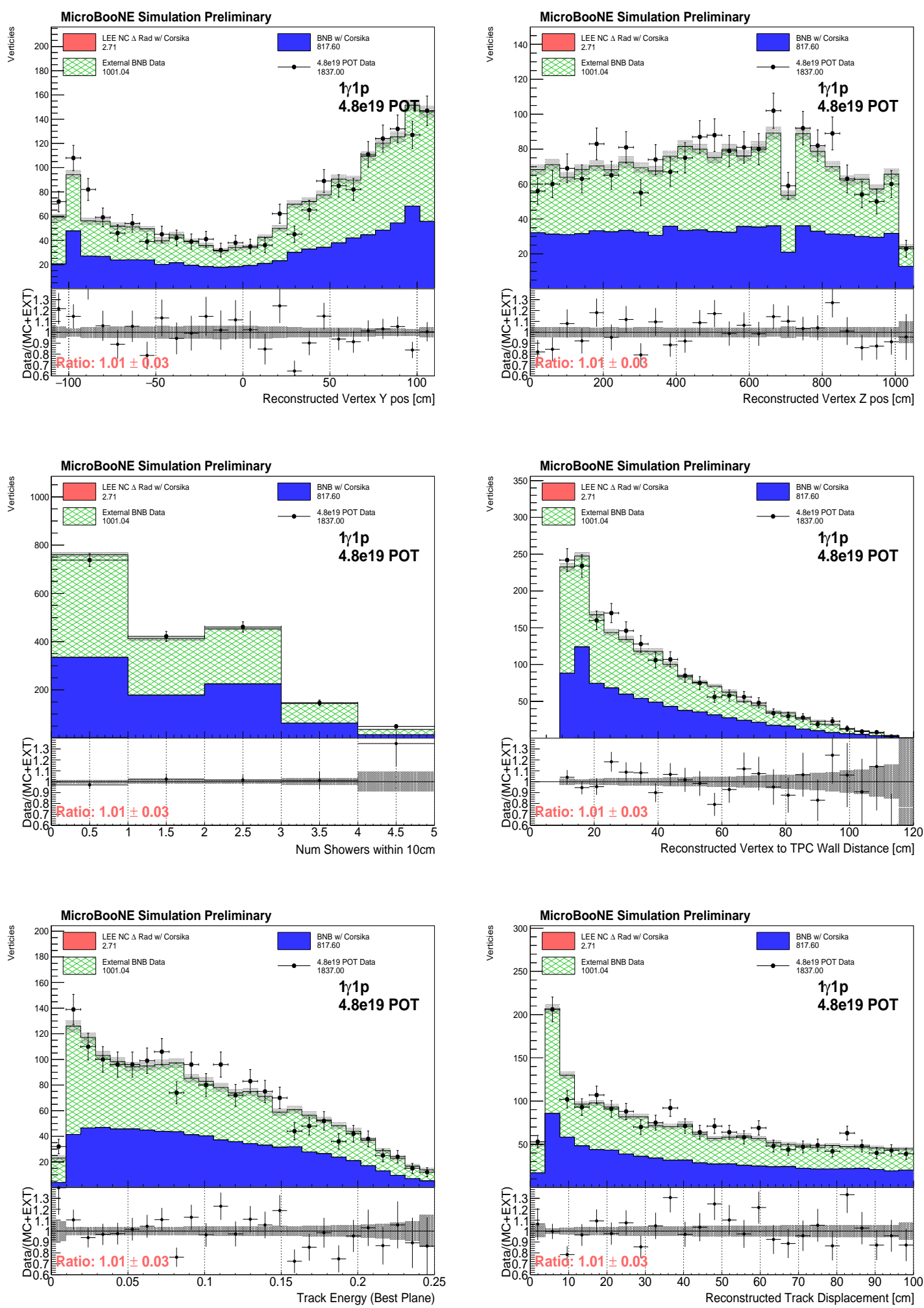

Figure 6.12: $1 \gamma 1 p$ data-MC+EXT comparison for several variables directly after pre-selection. The top panel contains the variable with the data superimposed on the $\mathrm{MC}+\mathrm{EXT}$ samples. The bottom panel contains the ratio of data to $\mathrm{MC}+\mathrm{EXT}$ per bin. Statistical data errors are included on the ratio points, statistical MC+EXT errors are shown in the gray bands. From top left to bottom right: vertex $y$-position, vertex $z$-position, number of showers within $10 \mathrm{~cm}$ of vertex, vertex-closest TPC wall distance, track energy and track length. 

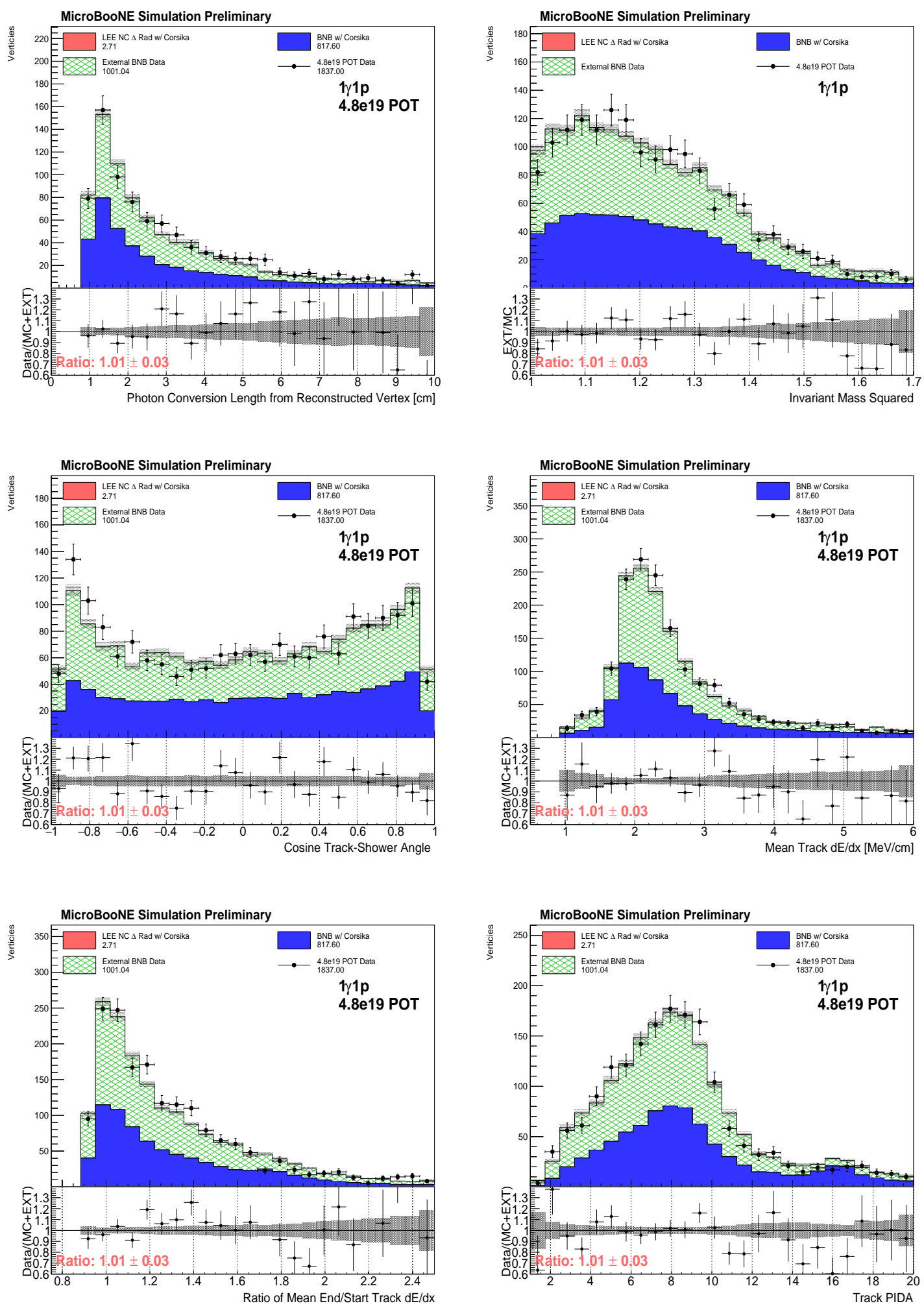

Figure 6.13: $1 \gamma 1 p$ data-MC+EXT comparison for several variables directly after preselection. The top panel contains the variable with the data superimposed on the $\mathrm{MC}+\mathrm{EXT}$ samples. The bottom panel contains the ratio of data to MC+EXT per bin. Statistical data errors are included on the ratio points, statistical MC+EXT errors are shown in the gray bands. From top left to bottom right: shower startvertex distance, track-shower invariant mass squared, cosine track-shower angle, mean track $\mathrm{dE} / \mathrm{dx}$, ratio of end-start mean track $\mathrm{dE} / \mathrm{dx}$ and track PIDA. 

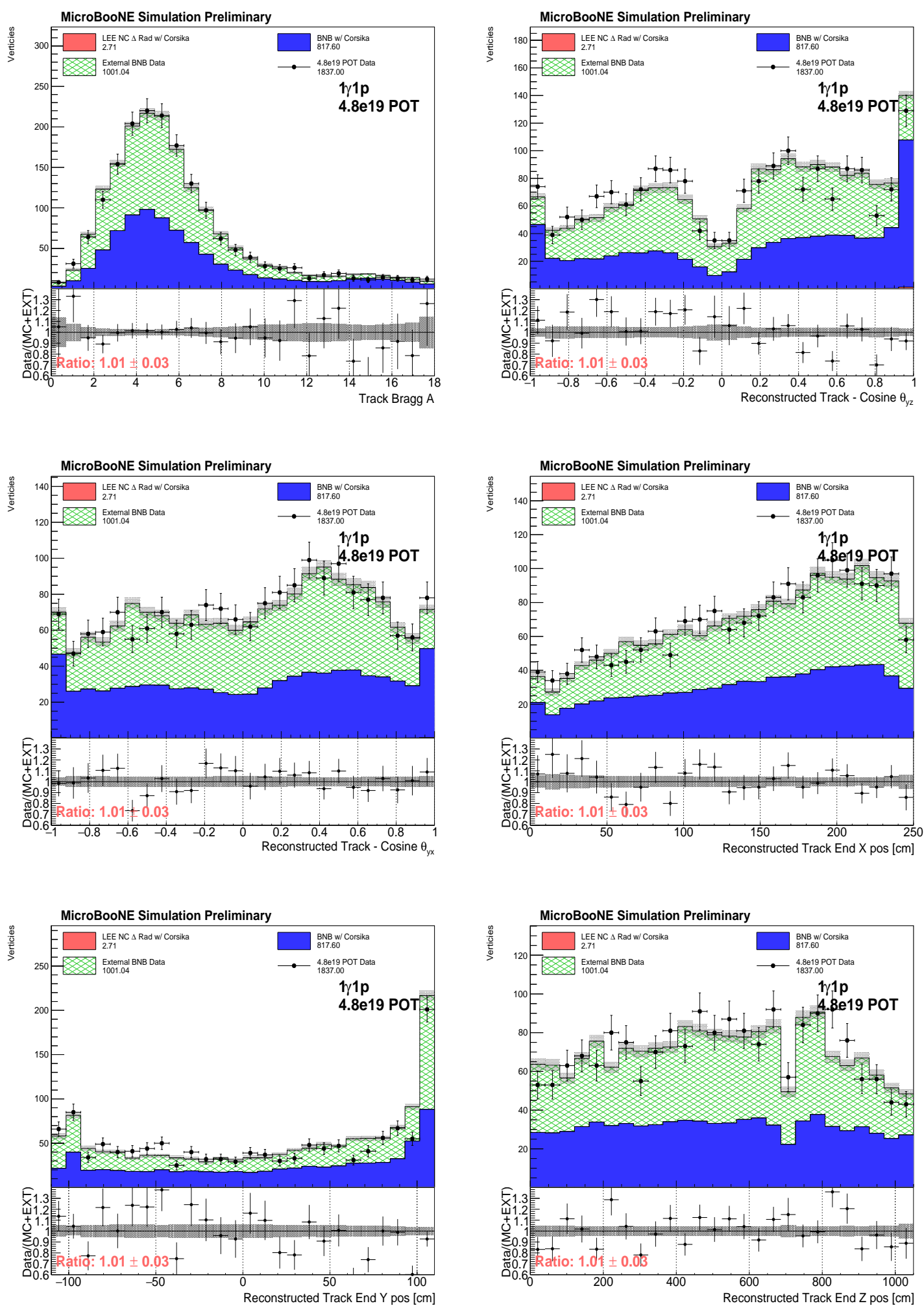

Figure 6.14: $1 \gamma 1 p$ data-MC+EXT comparison for several variables directly after pre-selection. The top panel contains the variable with the data superimposed on the MC+EXT samples. The bottom panel contains the ratio of data to MC+EXT per bin. Statistical data errors are included on the ratio points, statistical MC+EXT errors are shown in the gray bands. From top left to bottom right: track Bragg A value, track $\cos \theta_{y z}$, track $\cos \theta_{y x}$, track end $x$-position, track end $y$-position and track end $z$-position. 

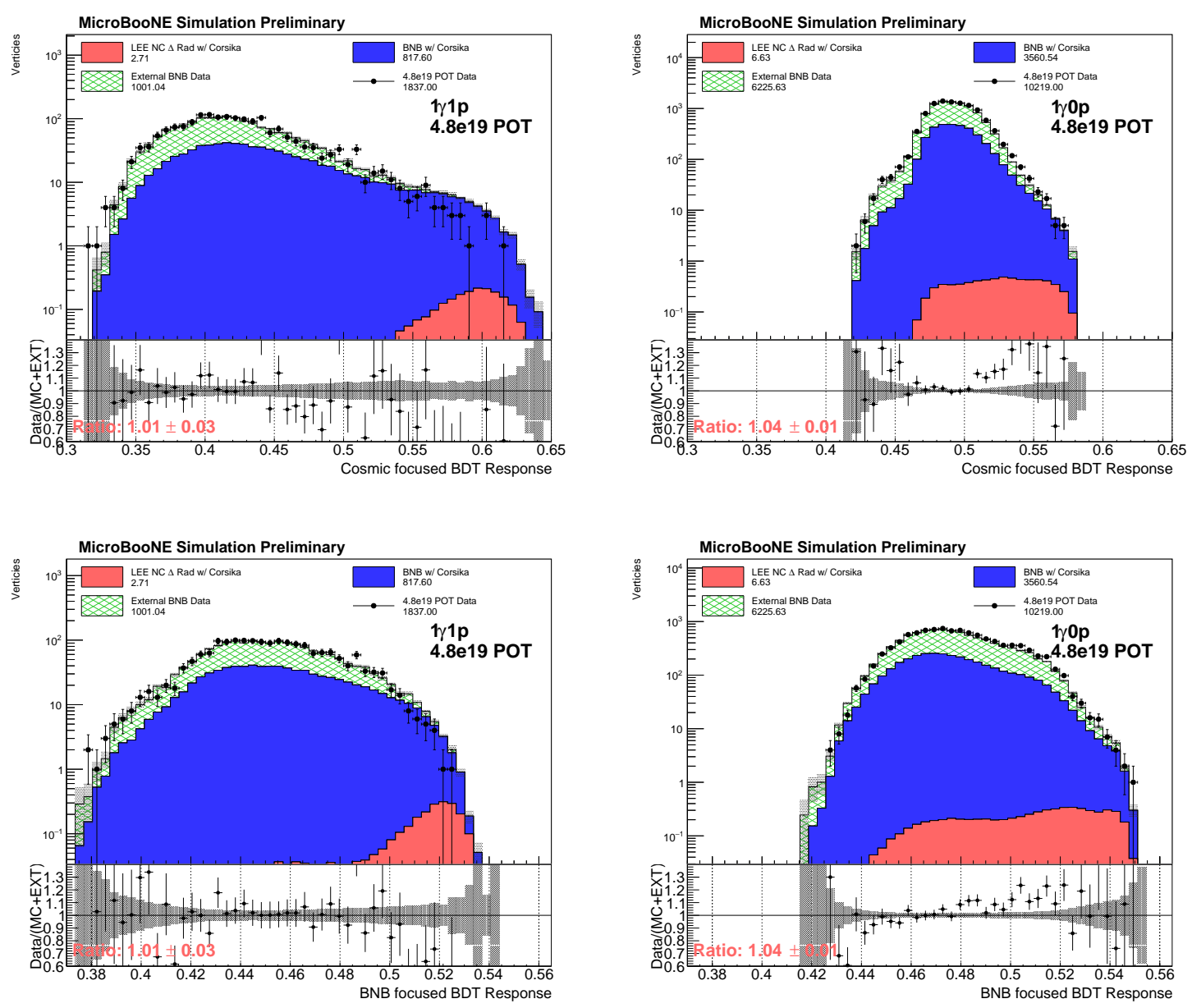

Figure 6.15: Data-MC+EXT comparison for each of the four background rejection BDT responses (after pre-selection). Top left: $1 \gamma 1 p$ cosmic BDT, top right: $1 \gamma 0 p$ cosmic BDT, bottom left: $1 \gamma 1 p$ BNB BDT, bottom right: $1 \gamma 0 p$ BNB BDT. Each plot is subdivided into two panels in the same manner as Figures 6.10 to 6.14 .

Figure 6.15 shows the data-MC+EXT comparison for each of the four background rejection BDTs after pre-selection cuts have been applied. The $1 \gamma 0 p \mathrm{NC} \Delta$ radiative + cosmic BDT response distributions (red in the two right plots of Figure 6.15) are spread over a relatively wide area and a large portion of the vertices in these distributions have low, background-like BDT responses for both the cosmic and BNB BDTs. These vertices are the CORSIKA cosmic component of the NC $\Delta$ radiative + cosmic sample that have not been eliminated by the pre-selection cuts. No cosmic vertices with low BDT responses are seen in the NC $\Delta$ radiative + cosmic sample for the $1 \gamma 1 p$ topology (red in the two left plots of Figure 6.15) as these vertices have already been removed by the additional track pre-selection cuts.

There are data-MC+EXT shape disagreements in both of the $1 \gamma 1 p$ BDTs (left plots in Figure 6.15), in the response area where the majority of the signal lies. Discrepancies in the track $\mathrm{dE} / \mathrm{dx}$ variables may point to a possible source of the 

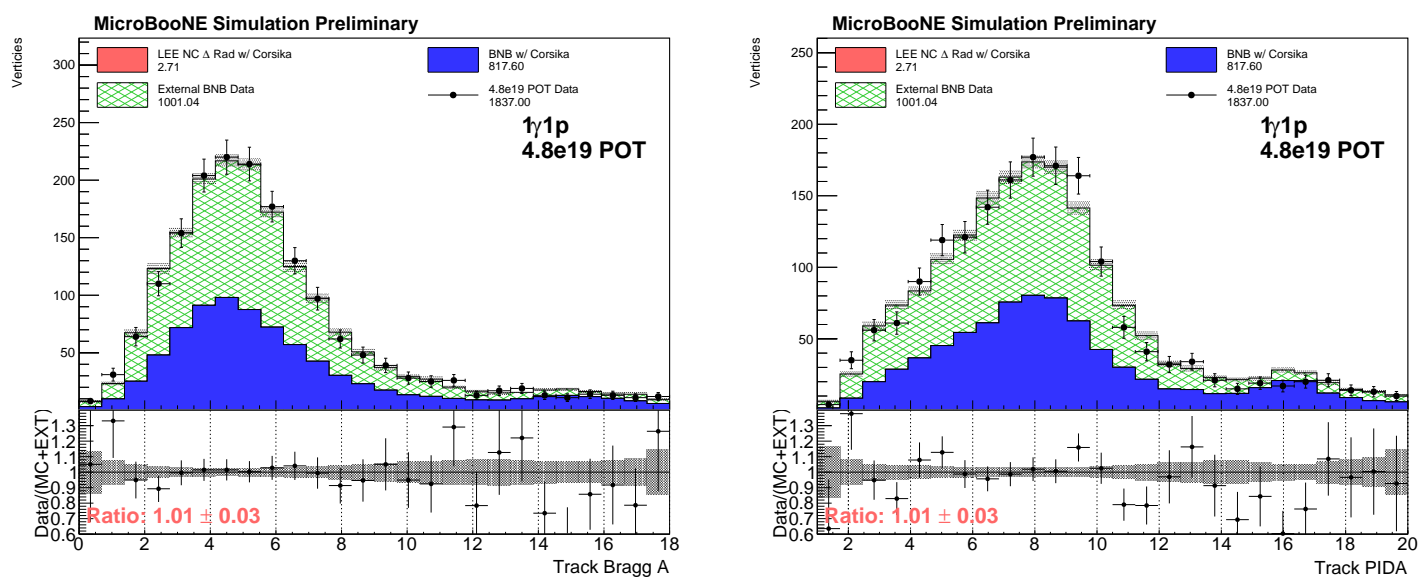

Figure 6.16: $1 \gamma 1 p$ data-MC+EXT comparison for the track Bragg A (left) and track PIDA (right) variables directly after pre-selection.
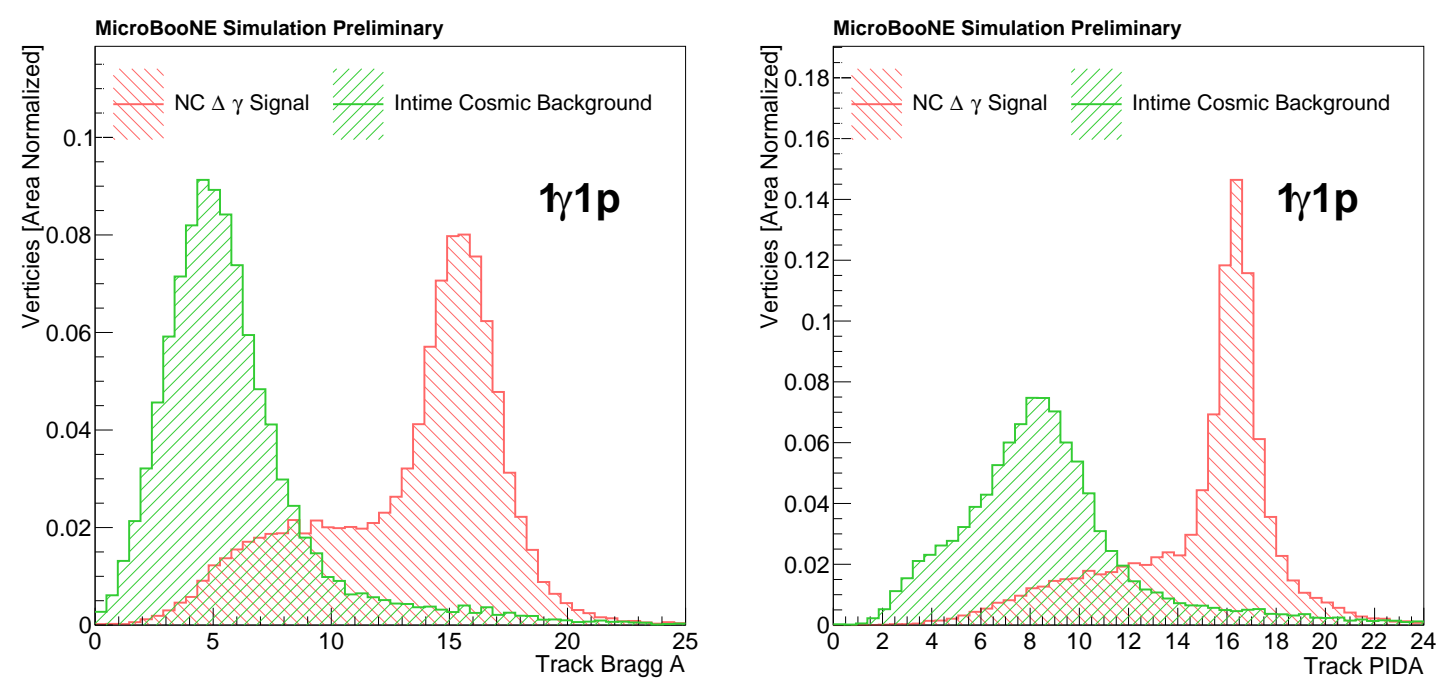

Figure 6.17: Track variable distributions used to train the $1 \gamma 1 p$ cosmic rejection BDT. Left: track Bragg A right: track PIDA.

BDT disagreements. One of these variables is track Bragg $A$, the data-MC+EXT comparison of which is shown in the left plot in Figure 6.16. At first glance the comparison shows relatively good agreement in both shape and normalization. However there is a small shape disagreement situated at $A \approx 15$. This area is exactly where protons are expected to appear and thus where the majority of the $1 \gamma 1 p$ NC $\Delta$ radiative reco-vertices reside, as can be seen in cosmic BDT training distributions for track Bragg $A$ in the red distribution of Figure 6.17. A similar situation is seen for the track PIDA variable, with a small shape disagreement in the right plot of Figure 6.16 at $\mathrm{PIDA} \approx 16$. This is again where the majority of the $1 \gamma 1 p \mathrm{NC} \Delta$ 
radiative reco-vertices are situated as can be seen in the right plot of Figure 6.17. Disagreements for similar track related variables in the regions where protons are expected to reside have been seen in other MicroBooNE studies [88] but the cause is not properly understood.

Data-MC+EXT shape disagreements can also be seen in the $1 \gamma 0 p$ BDTs (right plots in Figure 6.15). There are known differences between CORSIKA simulated cosmics (used in the $\mathrm{NC} \Delta$ radiative + cosmic and $\mathrm{BNB}+$ cosmic samples) and cosmic data in MicroBooNE which are attributed to CORSIKA mis-modeling. A cosmic-only data-MC comparison was performed between the BNB external cosmic data sample (EXT) and the CORSIKA generated in-time cosmic sample (MC, see Section 5.3p for the $1 \gamma 1 p$ training variables and the four BDT responses in the same manner as the data-MC+EXT comparison. This comparison can be found in Appendix A and will henceforth be referred to as the EXT-MC comparison. Substantial normalization differences can be seen between the BNB cosmic external data and the CORSIKA in-time sample for both the $1 \gamma 1 p$ and $1 \gamma 0 p$ topologies as well as shape differenced in some of the training variables (Figures A.1 to A.5) and the BDT responses (Figure A.6). Given the EXT-MC disagreements, the use of CORSIKA cosmics in the $\mathrm{NC} \Delta$ radiative + cosmic and $\mathrm{BNB}+$ cosmic samples is likely the source of the $1 \gamma 0 p$ Data-MC+EXT shape disagreements. Such disagreements can also be seen in the $1 \gamma 1 p$ BDT responses, however these disagreements are not as strongly reflected data-MC+EXT comparison for the $1 \gamma 1 p$ BDT responses. This could be due to the additional constraints placed on the $1 \gamma 1 p$ topology by the track-only pre-selection cuts if they remove vertices contributing to the shape disagreement.

Given the disagreement seen between CORSIKA simulated cosmics and cosmic data, MicroBooNE analyses have switched to the use of BNB external data to predict both BNB mimicking cosmic events (predicted in this analysis by the BNB external cosmic data sample) and the cosmic activity that is coincident with BNB neutrino interactions. Unfortunately, high statistics samples of $\mathrm{NC} \Delta$ radiative and BNB events with overlaid cosmic data were not available for this thesis and so CORSIKA was the only option for BNB coincident cosmic activity. Preliminary lower-statistics studies with cosmic data-only prediction samples have been performed for this analysis and show much better data-MC+EXT agreement compared to the comparison using CORSIKA cosmics [89]. As such, future iterations of this analysis will switch to using data-only cosmics in all of their prediction samples which should alleviate the data-MC+EXT shape disagreements seen in the $1 \gamma 0 p$ BDTs. 
Figures 6.18 and 6.19 shows the data-MC+EXT comparison for the $1 \gamma 0 p$ and $1 \gamma 1 p$ reconstructed shower energy variable after all selection stages have been applied (vertex reconstruction, pre-selection and cosmic and BNB BDT response cuts). The small size of the unblinded data sample means that post-selection there are very few events remaining. This is particularly true for the $1 \gamma 1 p$ topology which has even fewer reco-vertices remaining due to the additional constraints on the track variables placed by the selection. The data and MC+EXT appear to agree for the majority of bins that contain data reco-vertices but the low statistics at this stage make it difficult to draw any conclusions.

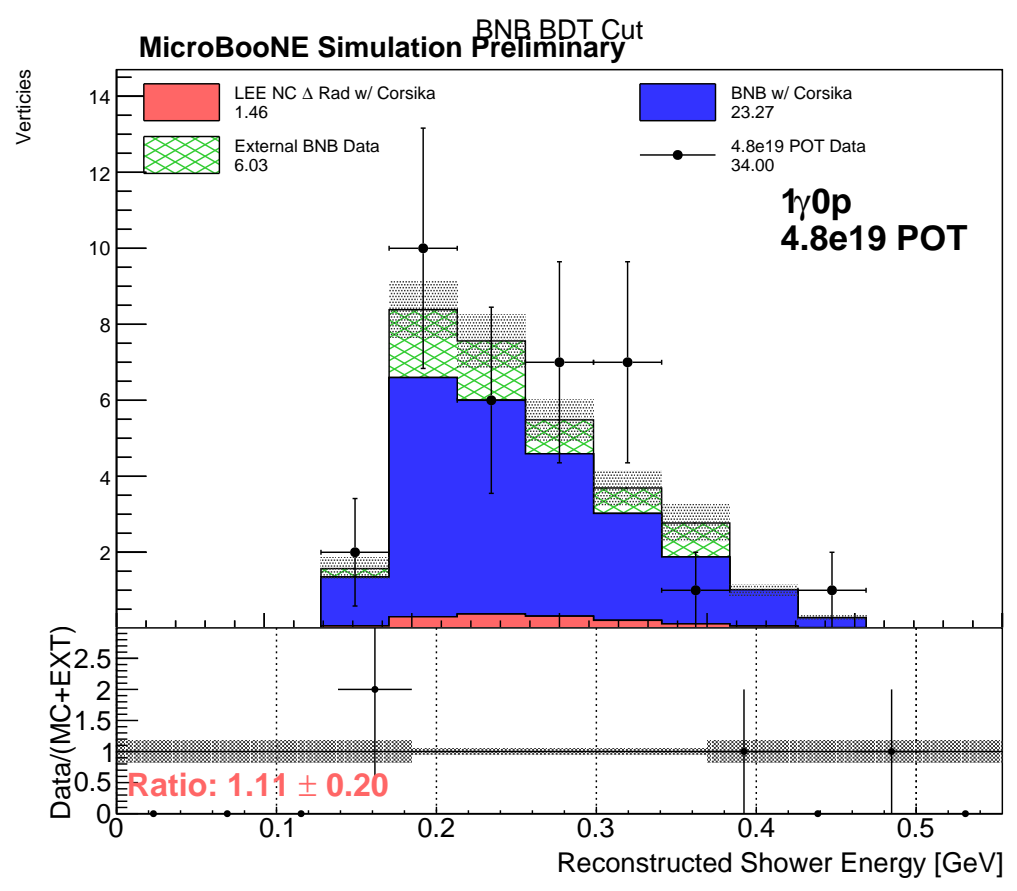

Figure 6.18: Data-MC+EXT comparison for the $1 \gamma 0 p$ reconstructed shower energy variable after all selection stages have been applied. 


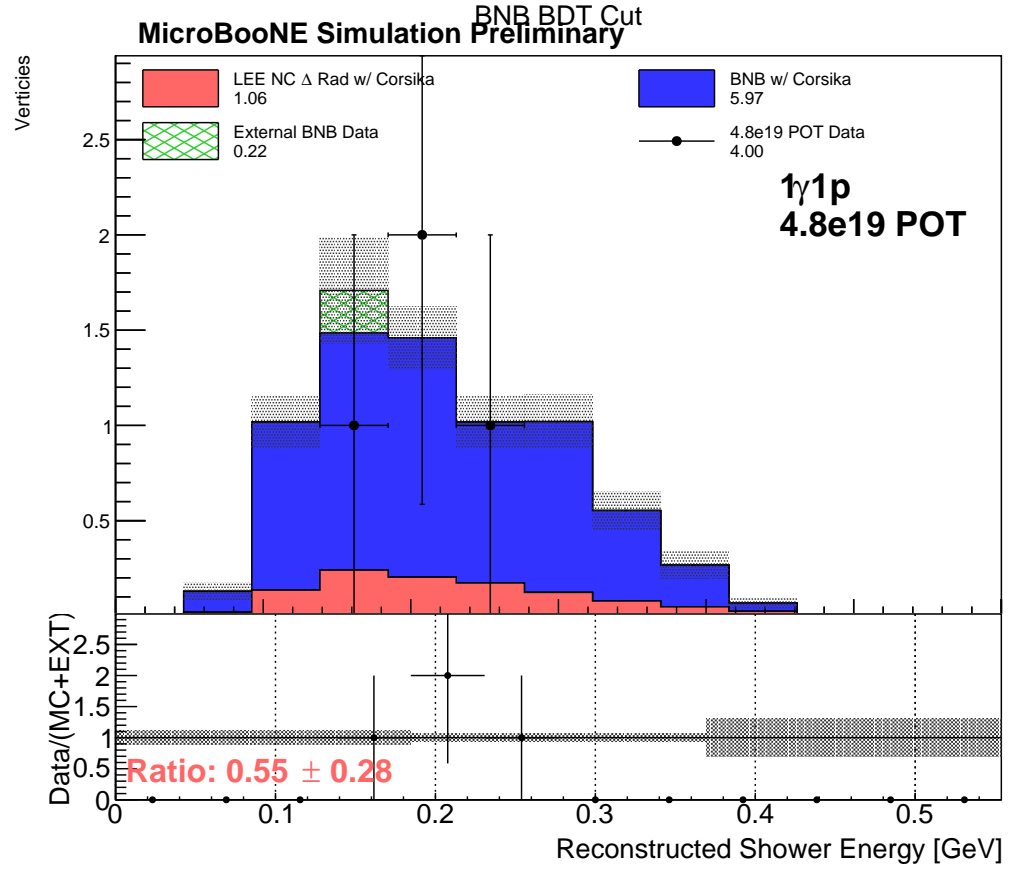

Figure 6.19: Data-MC+EXT comparison for the $1 \gamma 1 p$ reconstructed shower energy variable after all selection stages have been applied. 


\subsection{Systematic Uncertainties}

Proper handling of systematics is crucial to setting a realistic limit on neutrino induced NC $\Delta$ production and subsequent radiative decay in MicroBooNE. Unfortunately systematic uncertainties have not been applied to the results in this thesis. Potential contributing factors to the systematic uncertainty of the analysis will discussed here. The impact of BNB flux, theoretical modeling and detector systematic uncertainties on the BDT responses was discussed in Section 5.7. While overall rate changes do not impact the BDT responses they will impact the sensitivity of the analysis to $\mathrm{NC} \Delta$ radiative decay.

Uncertainties on the expected BNB neutrino flux at MicroBooNE were discussed in Section 3.1. Studies performed to determine the expected BNB neutrino flux at MicroBooNE place estimates of systematic uncertainty of the $\nu_{\mu}$ flux at $12.5 \%$. $\pi^{+} \rightarrow \mu^{+}+\nu+\mu$ decay is the main source of $\nu_{\mu}$ flux in the BNB and as such, the main contribution to the $\nu_{\mu}$ flux uncertainty is the production of $\pi^{+}$s from protonberyllium interactions in the BNB target at $11.7 \%$.

Theoretical modeling uncertainties were discussed in Subsection 4.1.2. The choice of model for NC $\Delta$ radiative decay will factor into the systematic uncertainty of the analysis, however estimates of this uncertainty have not yet been performed. FSIs are another potential source of uncertainty, with initial estimates from the MicroBooNE analyses placing the GENIE FSI systematic uncertainty at approximately $10 \%$. The cross-section studies performed in the SBN proposal estimated the GENIE cross-section uncertainty to be $20 \%$. While the overall GENIE cross-section uncertainties will affect all BNB related backgrounds in the analysis, certain GENIE parameters (and their uncertainties) are particularly relevant to the sensitivity of the analysis and were not included in the SBN studies. The first of these is the branching ratio for radiative resonance decays, $x_{B R}^{R \rightarrow X+1 \gamma}$ which GENIE places a 50 $\%$ uncertainty on. The second parameter is the pion charge exchange probability, $x_{c e x}^{\pi}$. A charged pion produced in the nucleus produced by $\Delta \rightarrow n+\pi$ decay can exchange charge with a nucleon in the nucleus resulting in the production of a $\pi^{0}$ and thus contribute to background. GENIE places the relative uncertainty of $x_{c e x}^{\pi}$ at $50 \%$.

Detector modeling uncertainties were discussed in Subsection 3.2.4. The initial estimates of detector systematics from MicroBooNE analyses place the total detector systematic uncertainty at approximately $20 \%$. While the systematic uncertainties listed for the analysis are relatively high, they can be somewhat constrained by 
performing an in-situ measurement of $\mathrm{NC} \pi^{0} \rightarrow 2 \gamma$ decay as was done in MiniBooNE.

\subsection{Sensitivity to a NC $\Delta$ radiative Low Energy Excess}

Figures 6.20 shows MicroBooNE's expected statistical errors-only exclusion limit for $\mathrm{NC}$ neutrino-induced $\Delta$ radiative photon production as a function of crosssection enhancement. The expected exclusion limit was determined with the CLs method [90], using the post-selection $1 \gamma 0 p$ and $1 \gamma 1 p$ shower energy distribution predictions scaled to $6.6 \times 10^{20} \mathrm{POT}$ in a side-by-side fit. Enhancing the NC $\Delta$ radiative cross-section to match the MiniBooNE LEE (denoted by the black line) shows that, when only considering statistical uncertainty, MicroBooNE is currently insensitive to a NC $\Delta$ radiative produced LEE. The median MicroBooNE experiment is able to set a 99\% CL limit for NC $\Delta$ radiative photon production if the standard GENIE-predicted cross-section is enhanced by $\times 4.6$. This corresponds to a $\times 1.5$ enhancement for the $\mathrm{NC} \Delta$ radiative LEE cross-section.

Figure 6.21 shows MicroBooNE's expected exclusion limit for LEE $(\times 3)$ crosssection enhanced NC neutrino-induced $\Delta$ radiative photon production as a function of additional background rejection. This exclusion limit was determined using the CLs method and the same distributions as the previous limit. If the post-selection background can be reduced by a further factor of 2.2, the median MicroBooNE experiment will be able to place a $99 \% \mathrm{CL}$ limit for a NC $\Delta$ radiative LEE. The dominant background for the analysis is NC $\pi^{0} \rightarrow 2 \gamma$ decay and as was discussed in Section 6.2, a SSN could be trained to identify the activity of a missed second shower and allow for significant reduction in such cases. As these cases account for $53 \%$ of the $1 \gamma 1 p$ NC $\pi^{0}$ background (see Table 6.5) and assuming minimal signal efficiency loss using this approach, a significant reduction of post-selection background is achievable.

In addition to this, improvements to reconstruction in MicroBooNE are ongoing. Improvements to shower energy and $\mathrm{dE} / \mathrm{dx}$ reconstruction could yield further improvements at the BDT stage of event selection. Improved shower energy and $\mathrm{dE} / \mathrm{dx}$ training variables (and other variables in general) could produce more powerful optimum cuts to be placed in earlier decision nodes in the BDT training, allowing for the subsequent placement of more powerful cuts for different variables further down the decision tree and improving BDT separation. Improvements to reconstructed shower direction would reduce the uncertainty on shower backwards-projection and 
association performed by the vertex reconstruction algorithm. Such improvements would improve vertex completeness, an important quality for $\pi^{0} \rightarrow 2 \gamma$ background mitigation and the correct association of $\Delta^{+} \rightarrow p+\gamma$ photon showers with their corresponding proton tracks. Improvements to completeness could also allow for the reduction of the vertex algorithm input parameters, increasing vertex cleanliness and thus reducing the number of signal vertices lost by the erroneous association of additional showers or tracks. Such improvements would likely also be beneficial at the BDT training stage, again improving BDT separation. Considering such potential improvements in addition to the reduction of the NC $\pi^{0}$ background by a SSN, reduction of the post-selection background by a further factor of 2.2 is feasible.

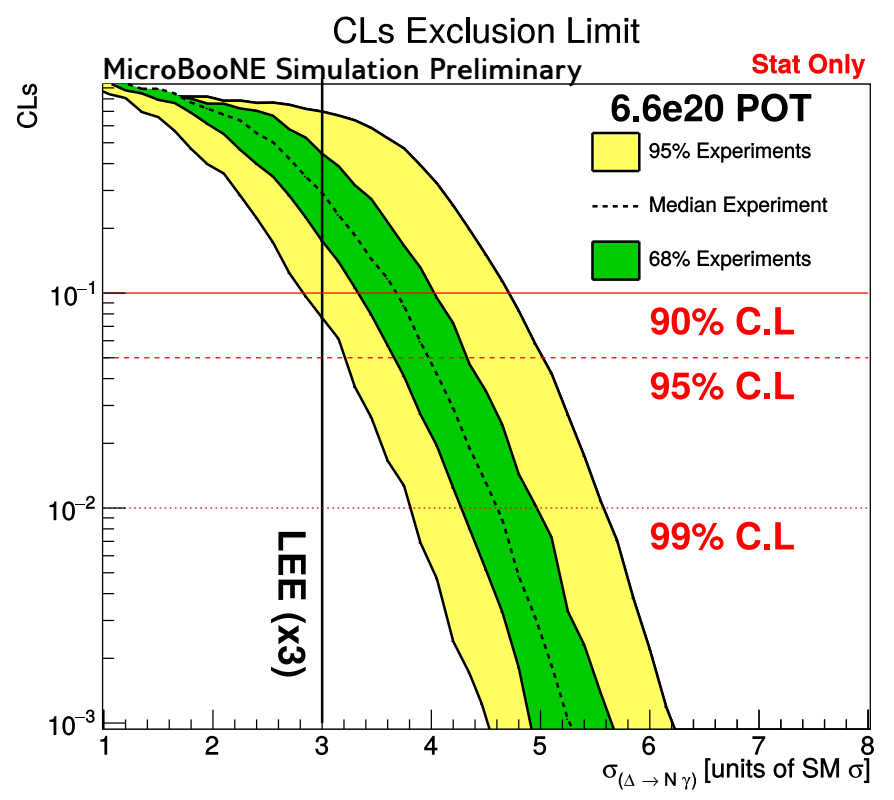

Figure 6.20: MicroBooNE's statistical errors-only expected exclusion limit for NC $\Delta$ radiative photon production as a function of cross-section enhancement for $6.6 \times$ $10^{20}$ POT. The cross-section enhancement required to reach the magnitude of the MiniBooNE LEE is denoted with a black line [78]. 


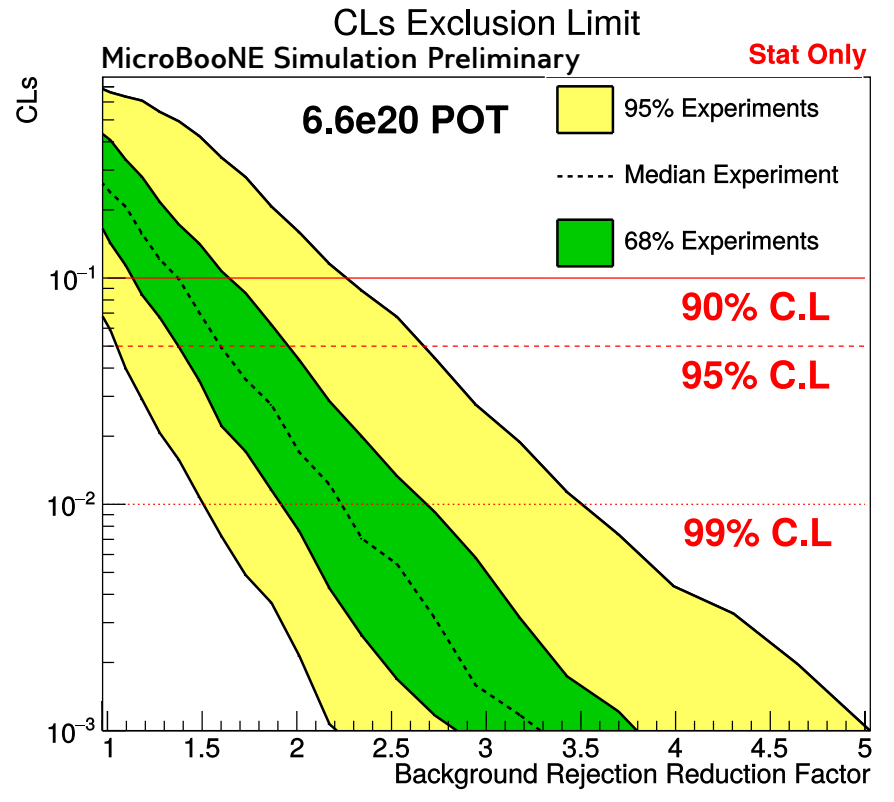

Figure 6.21: MicroBooNE's statistical errors-only expected exclusion limit for LEE $(\times 3)$ cross-section enhanced $\mathrm{NC} \Delta$ radiative photon production as a function of additional background rejection for $6.6 \times 10^{20}$ POT $[78]$. 


\section{Conclusions}

An excess of $\nu_{e}$ CCQE event candidates in the reconstructed $E_{\nu}^{Q E} \approx 200-475 \mathrm{MeV}$ region was observed in the MiniBooNE detector. The inability of the detector to distinguish between photons and electrons means identity of this excess as photon or electron is unknown. The ability to distinguish between photon and electrons and a location on the same beamline and similar baseline as MiniBooNE make MicroBooNE the ideal experiment to perform a Low Energy Excess (LEE) search and subsequently determine the source of the LEE. The analysis in this thesis considers the photon interpretation of the LEE using a $\times 3$ enhanced rate of neutrino-induced $\mathrm{NC} \Delta$ radiative decay as a candidate source of the excess.

A multi-stage neutrino-induced NC $\Delta \rightarrow N+\gamma$ decay selection has been developed in MicroBooNE for the purposes of detecting a photon LEE. This selection takes advantage of the shower $\mathrm{dE} / \mathrm{dx}$ photon-electron differentiation capability in MicroBooNE as well as photon conversion length (with a well defined vertex) to reduce the BNB intrinsic $\nu_{e}$ by $99.5 \%$ and completely remove the BNB Michel electron background. The first stage of this selection is a vertex reconstruction algorithm designed to reconstruct single-photon neutrino interaction vertices. The vertex algorithm input parameters have been optimized to select single photon events giving the vertex algorithm improved performance over the standard Pandora neutrino vertex reconstruction for single photon events in terms of combined completeness and cleanliness. The second stage sees the reconstructed vertices from the first stage separated into two distinct topologies: the lone shower topology $1 \gamma 0 p$ and the one shower-one track topology $1 \gamma 1 p$ before being subject to a series of pre-selection cuts designed primarily to ensure the quality of reconstructed objects associated with the vertex and to mitigate the substantial cosmic background present in the analysis. The third and final stage takes the surviving vertices and applies a distinct cosmic and BNB background rejection BDT for both vertex topologies (a total of four BDTs).

Despite the rarity of neutrino-induced $\mathrm{NC} \Delta$ radiative decay in MicroBooNE (189 in-TPC events per $6.6 \times 10^{20}$ POT for $\times 3$ LEE enhancement) and the prevalence of backgrounds, the selection is able to select events with a total efficiency of $9 \%$, giving a combined expected statistical errors-only significance of $1.87 \sigma$ for $6.6 \times 10^{20} \mathrm{POT}$ of data. In terms of expected statistical errors-only sensitivity Mi- 
croBooNE is currently insensitive to a $\mathrm{NC} \Delta$ radiative LEE, however if the current background post-selection can be reduced by an additional factor of 2.2 then the median MicroBooNE experiment will be able to set a $99 \%$ CL exclusion limit on a NC $\Delta \rightarrow N+\gamma$ LEE. For this reason, improvements to the selection should be primarily focused on further reducing the post-selection background. The fact that the majority of the remaining background is produced by $\mathrm{NC} \pi^{0}$ make this a challenging prospect, however, due to the kinematic similarities between this background and NC $\Delta \rightarrow N+\gamma$. Given the most powerful differentiator for the NC $\pi^{0}$ background is the second shower, the SSN approach of looking for additional photon-like activity that could have been missed by reconstruction shows great potential in reducing background. This approach in addition to potential improvements to MicroBooNE reconstruction make the reduction of the post-selection background by an additional factor of 2.2 a feasible goal.

The comparison between MC+EXT and the $4.8 \times 10^{19}$ POT of unblinded MicroBooNE data for the selection showed mostly good agreement in both shape and overall normalization. The discrepancies seen in the $1 \gamma 0 p$ BDT responses should be alleviated by the switch from CORSIKA simulated cosmics to cosmic data. The discrepancies seen in the signal regions of the $1 \gamma 1 p$ BDT responses require further study.

There are numerous potential contributions to the systematic uncertainty of the search, particularly from parameters relating to $\mathrm{NC} \pi^{0}$ production. While a proper run-through of systematic uncertainties is required, an in-situ measurement of the NC $\pi^{0}$ rate in MicroBooNE will likely help to significantly constrain some of these uncertainties.

In summary the search for a NC $\Delta \rightarrow N+\gamma$ LEE in MicroBooNE is a challenging one due to the low signal event rate, high cosmic rate and the persistent $\mathrm{NC}$ $\pi^{0}$ backgrounds. Significant progress has been made in developing a selection and MicroBooNE's ability to distinguish electrons and photons has been fully taken advantage of to reject the electron shower background. There is potential for significant improvement to analysis sensitivity should background rejection improve. 


\section{Appendix A}

\section{Cosmic-only Data-Monte Carlo Com- parison}

There are known differences between CORSIKA simulated cosmics and cosmic data in MicroBooNE which are attributed to CORSIKA mis-modeling. A cosmic-only data-MC comparison was performed between the BNB external cosmic data sample (EXT) and the CORSIKA generated in-time cosmic sample (MC, see Section 5.3) in order to study these differences. Figures A.1 to A.5 show the EXT-MC comparisons for the $1 \gamma 1 p$ BDT training variables after pre-selection cuts have been applied. Figure 6.15 shows the EXT-MC comparison for each of the four background rejection BDTs after pre-selection cuts have been applied. 

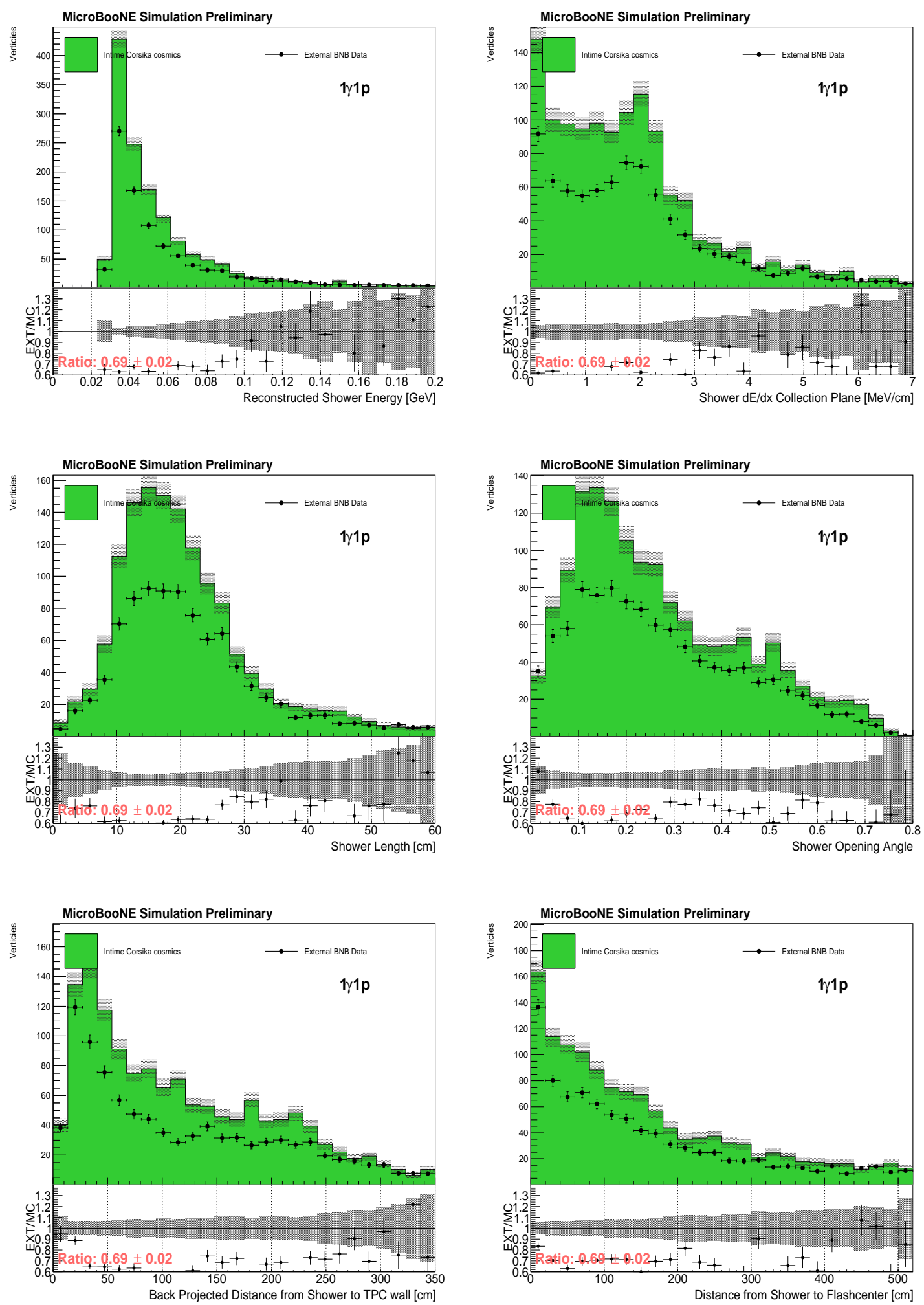

Figure A.1: $1 \gamma 1 p$ cosmic BNB external cosmic data (EXT) versus simulated in-time cosmic (MC) comparison for several variables directly after pre-selection. The top panel contains the variable with EXT superimposed onto MC. The bottom panel contains the ratio of EXT to MC per bin. Statistical EXT errors are included on the ratio points, statistical MC errors are shown in the gray bands. From top left to bottom right: shower energy, shower $\mathrm{dE} / \mathrm{dx}$, shower length, shower opening angle, backwards-projected shower distance from TPC wall and distance between shower and closest flash ( $z$-axis). 

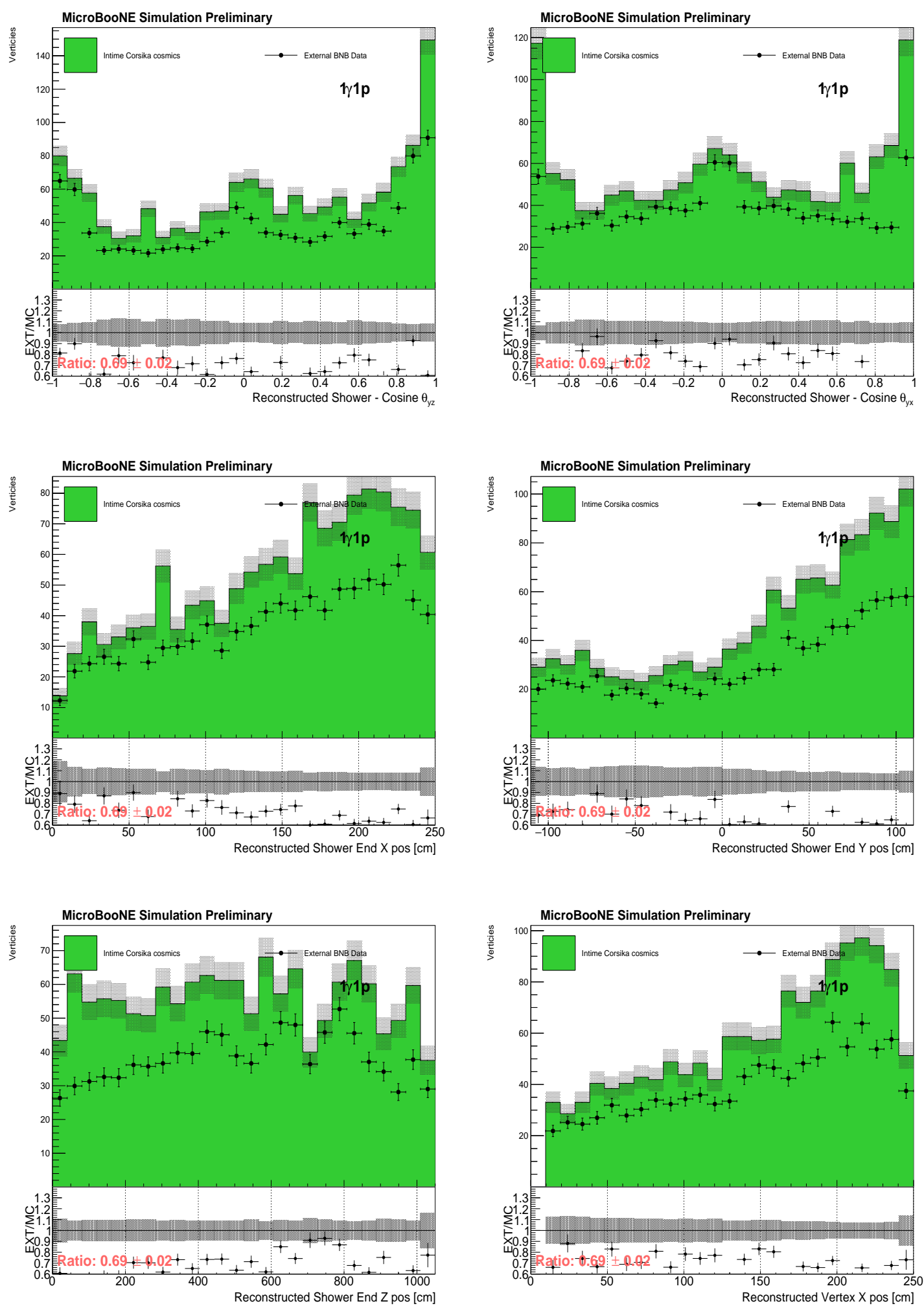

Figure A.2: $1 \gamma 1 p$ cosmic BNB external cosmic data (EXT) versus simulated in-time cosmic (MC) comparison for several variables directly after pre-selection. The top panel contains the variable with EXT superimposed onto MC. The bottom panel contains the ratio of EXT to MC per bin. Statistical EXT errors are included on the ratio points, statistical MC errors are shown in the gray bands. From top left to bottom right: shower $\cos \theta_{y z}$, shower $\cos \theta_{y x}$, shower end $x$-position, shower end $y$-position, shower end $z$-position and vertex $x$-position. 

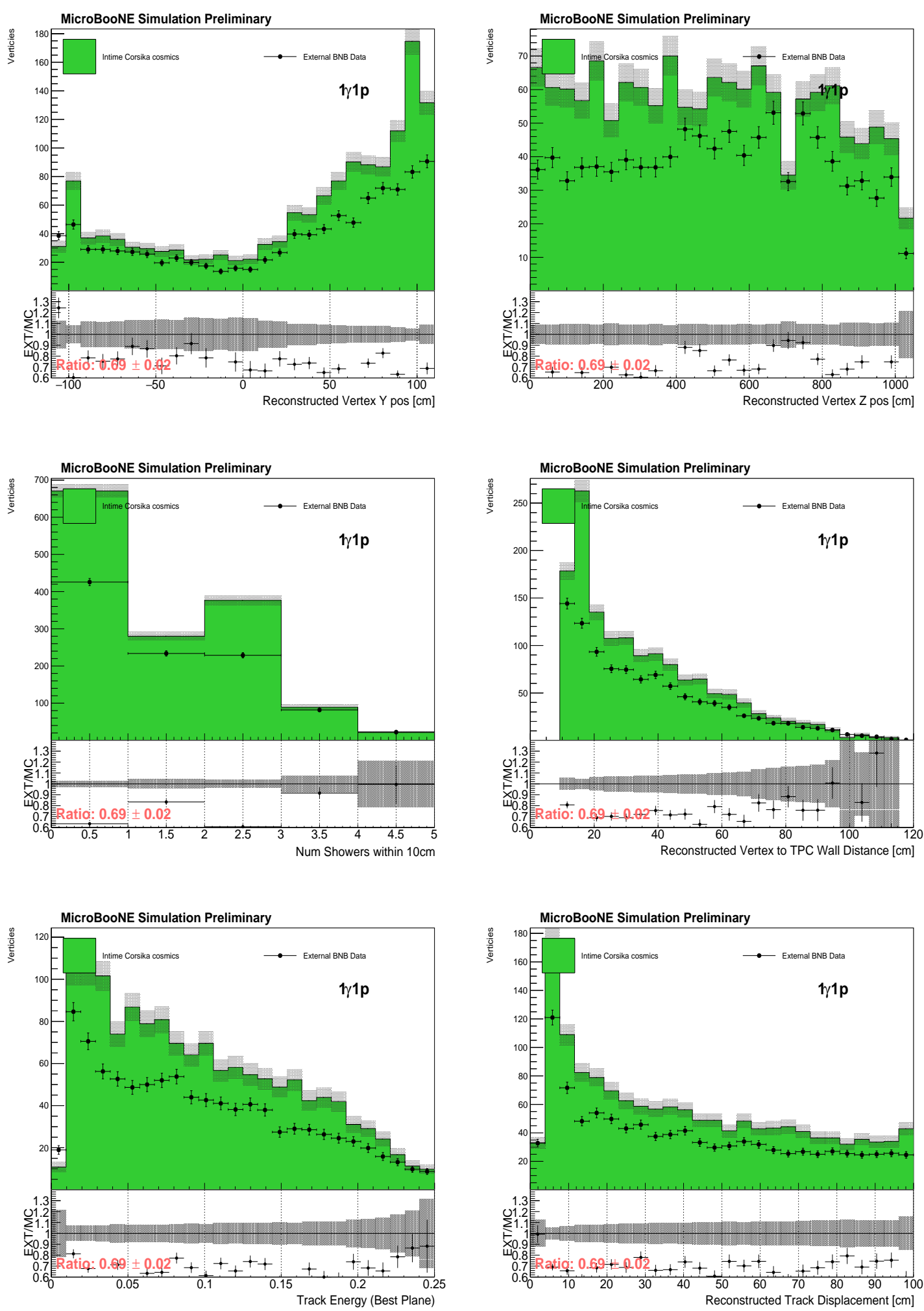

Figure A.3: $1 \gamma 1 p$ cosmic BNB external cosmic data (EXT) versus simulated in-time cosmic (MC) comparison for several variables directly after pre-selection. The top panel contains the variable with EXT superimposed onto MC. The bottom panel contains the ratio of EXT to MC per bin. Statistical EXT errors are included on the ratio points, statistical MC errors are shown in the gray bands. From top left to bottom right: vertex $y$-position, vertex $z$-position, number of showers within $10 \mathrm{~cm}$ of vertex, vertex-closest TPC wall distance, track energy and track length. 

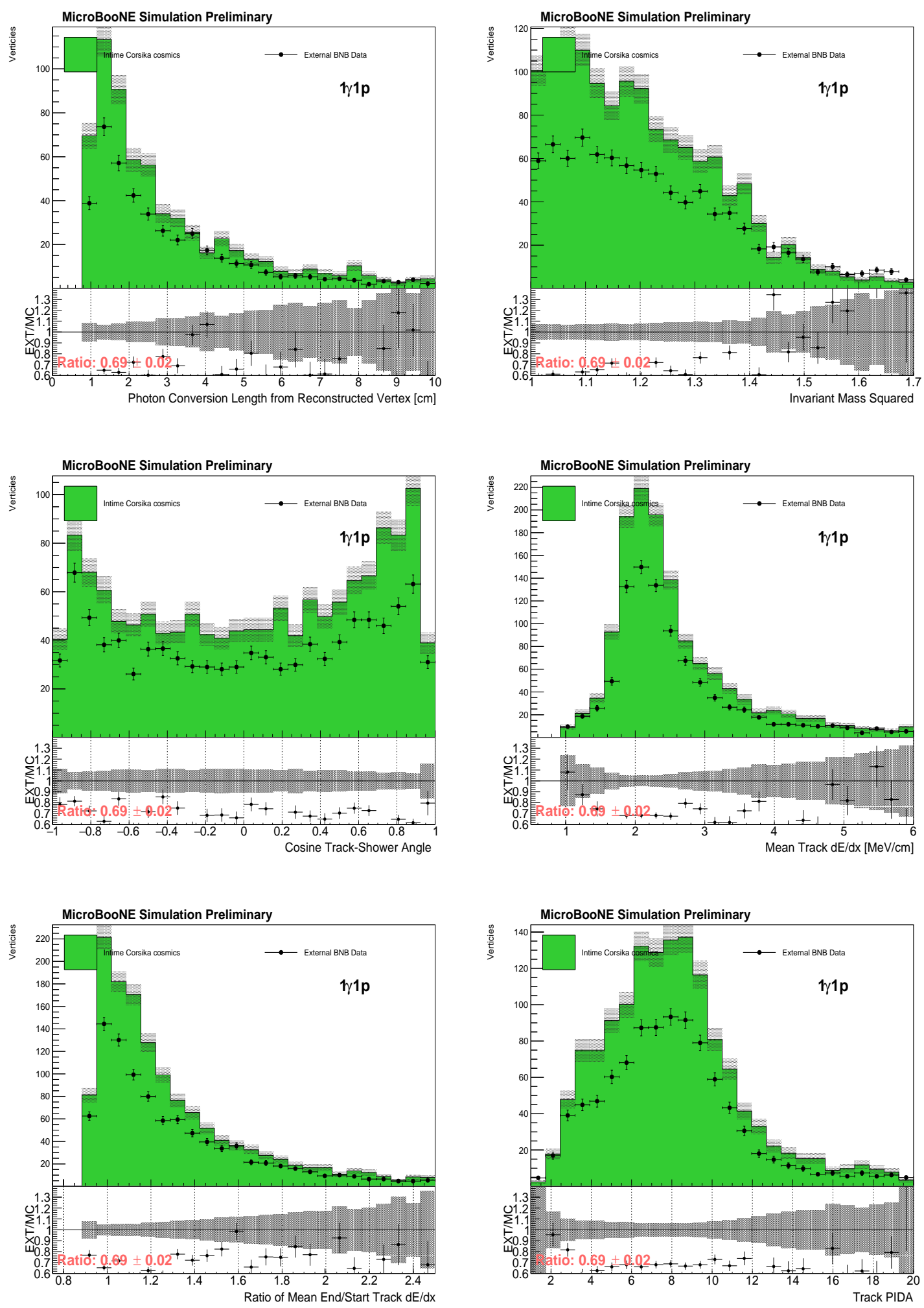

Figure A.4: $1 \gamma 1 p$ cosmic BNB external cosmic data (EXT) versus simulated in-time cosmic (MC) comparison for several variables directly after pre-selection. The top panel contains the variable with EXT superimposed onto MC. The bottom panel contains the ratio of EXT to MC per bin. Statistical EXT errors are included on the ratio points, statistical MC errors are shown in the gray bands. From top left to bottom right: shower start-vertex distance, track-shower invariant mass squared, cosine track-shower angle, mean track $\mathrm{dE} / \mathrm{dx}$, ratio of end-start mean track $\mathrm{dE} / \mathrm{dx}$ and track PIDA. 

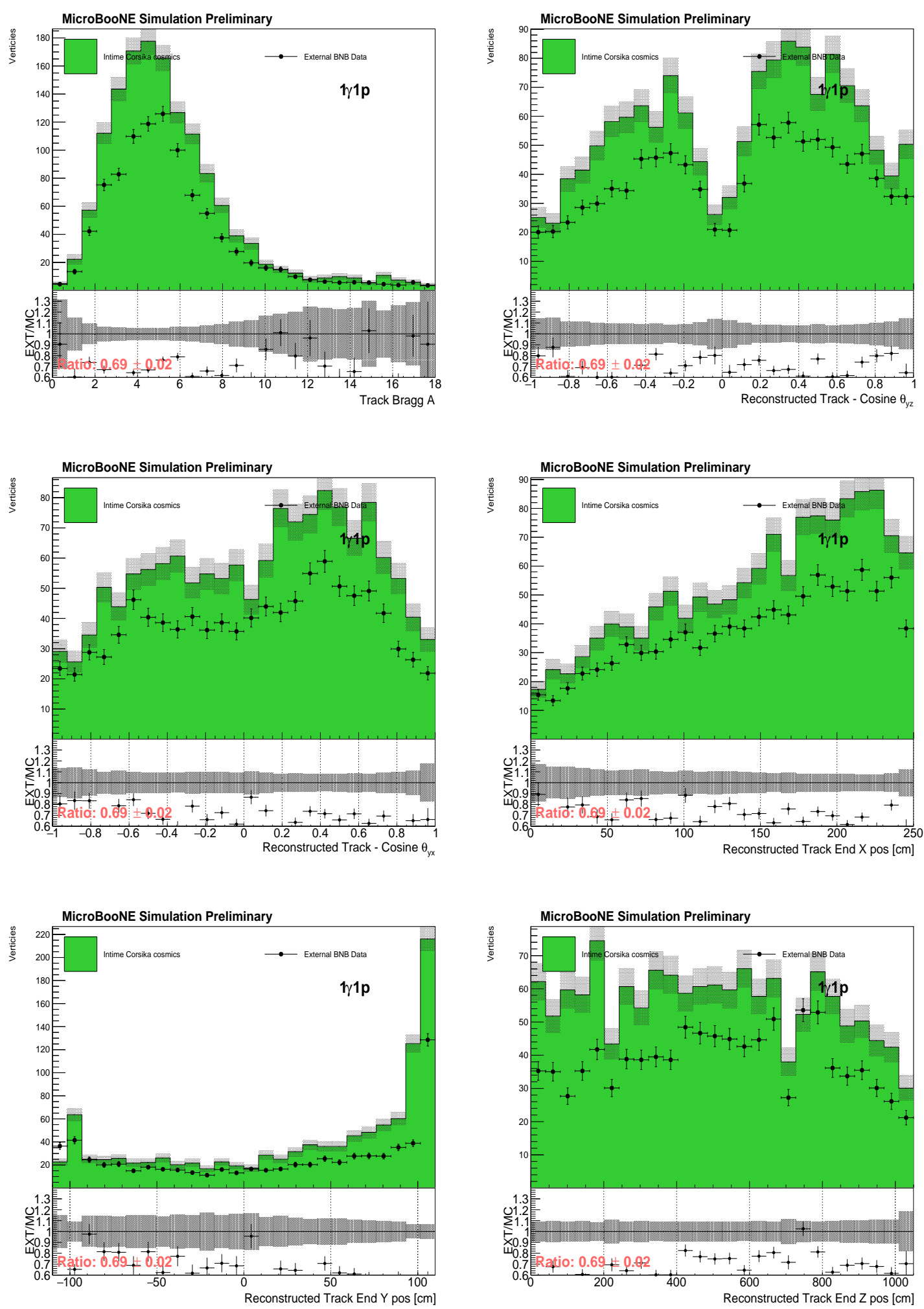

Figure A.5: $1 \gamma 1 p$ cosmic BNB external cosmic data (EXT) versus simulated in-time cosmic (MC) comparison for several variables directly after pre-selection. The top panel contains the variable with EXT superimposed onto MC. The bottom panel contains the ratio of EXT to MC per bin. Statistical EXT errors are included on the ratio points, statistical MC errors are shown in the gray bands. From top left to bottom right: track Bragg A value, track $\cos \theta_{y z}$, track $\cos \theta_{y x}$, track end $x$-position, track end $y$-position and track end $z$-position. 

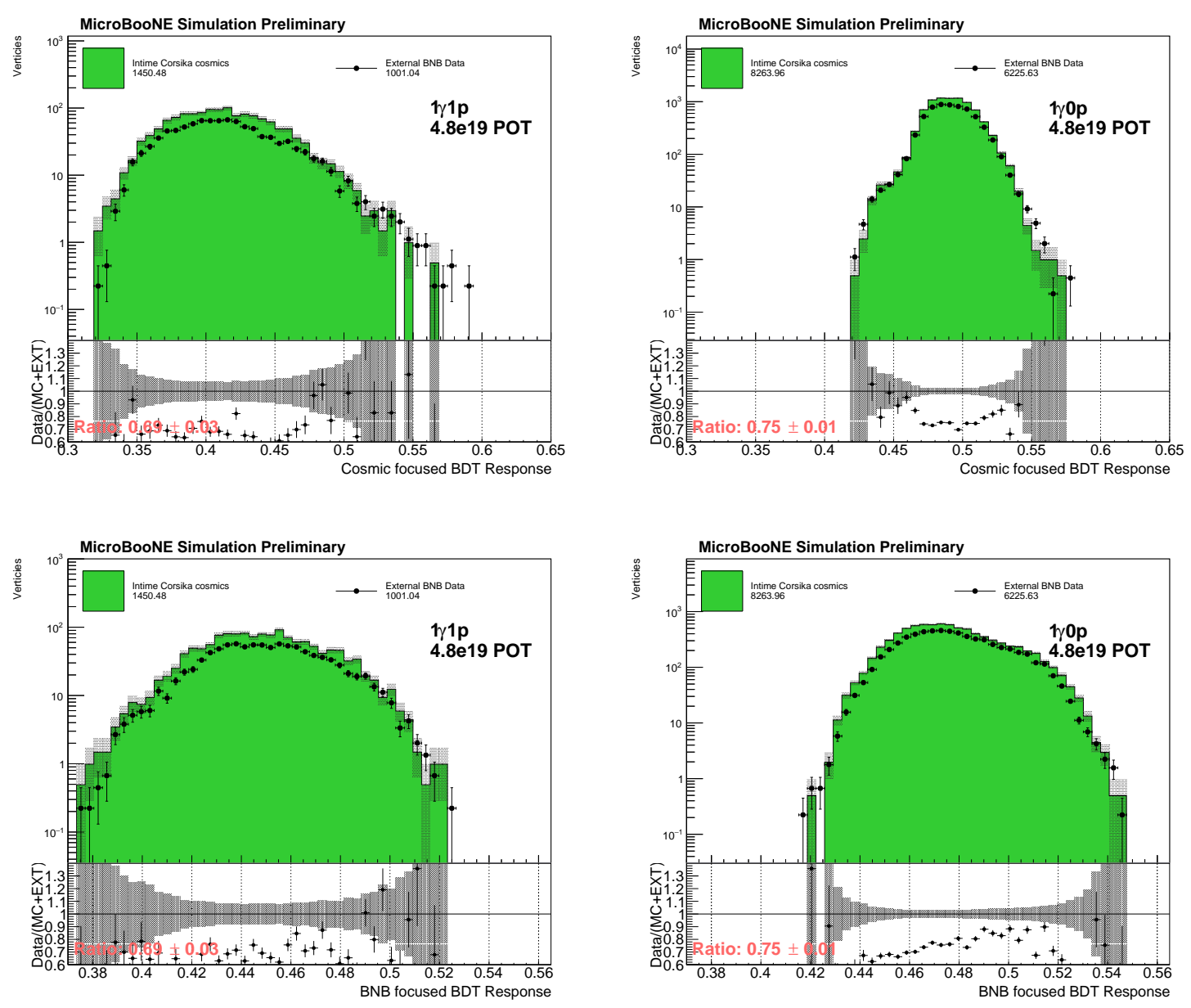

Figure A.6: Data-MC+EXT comparison for each of the four background rejection BDT responses (after pre-selection). Top left: $1 \gamma 1 p$ cosmic BDT, top right: $1 \gamma 0 p$ cosmic BDT, bottom left: $1 \gamma 1 p$ BNB BDT, bottom right: $1 \gamma 0 p$ BNB BDT. Each plot is subdivided into two panels in the same manner as Figures A.1 to A.5. 


\section{Appendix B}

\section{MiniBooNE Flux Cross-checks}

From 2003-2008 MiniBooNE took the data that would be used to observe the LEE. In November 2015 MiniBooNE started to take new data. The reasons for this revival were three-fold. The first was a cross-check to ensure the state of the BNB (and by extension the MiniBooNE detector) was the same as for the previous MiniBooNE run periods by isolating and comparing $\nu_{\mu} \mathrm{CCQE}$ event candidates in MiniBooNE from the old and new data. The second was to use the detector as a BNB rate monitor for MicroBooNE by measuring the number of $\nu_{\mu}$ candidates over the MicroBooNE run periods. The third was to collect more data and build a more robust LEE result (which was recently published [23]). With respect to the LEE searches in MicroBooNE, this is an extremely important cross-check to perform. Changes to the state of the BNB since the observation of the MiniBooNE LEE could impact the observation of a LEE in MicroBooNE. This appendix describes cross-check work performed for MiniBooNE and includes the operating principles of the MiniBooNE detector, the selection cuts used to isolate $\nu_{\mu}$ CCQE event candidates and the crosschecks performed between a 2003-2008 data sample and the first of the new data taken from 2015-2016.

\section{B.1 Detector}

The MiniBooNE detector is a Cherenkov based detector designed to search for $\nu_{\mu} \rightarrow \nu_{e}$ neutrino flavour transitions in the same $\Delta m^{2} \approx 1 \mathrm{eV}^{2}$ region as the LSND anomaly [86]. The detector is comprised of a $12.2 \mathrm{~m}$ diameter sphere containing 818 tons of mineral oil that acts as the neutrino target. The sphere is lined with 1280 inward-facing PMTs (the majority of which were taken from the LSND experiment) for coverage of the signal region of the detector. A $35 \mathrm{~cm}$ thick veto region designed for cosmic rejection surrounds the signal region and is covered by an additional 240 PMTs. The signal and veto regions are separated by an opaque barrier which prevents light from either region contaminating the other. The detector is situated on the BNB, $541 \mathrm{~m}$ downstream of the beryllium target, underneath a $3 \mathrm{~m}$ earth overburden. Figure B.1 shows the detector in its enclosure on the left and a cutaway of the detector revealing its signal and veto regions on the right. 

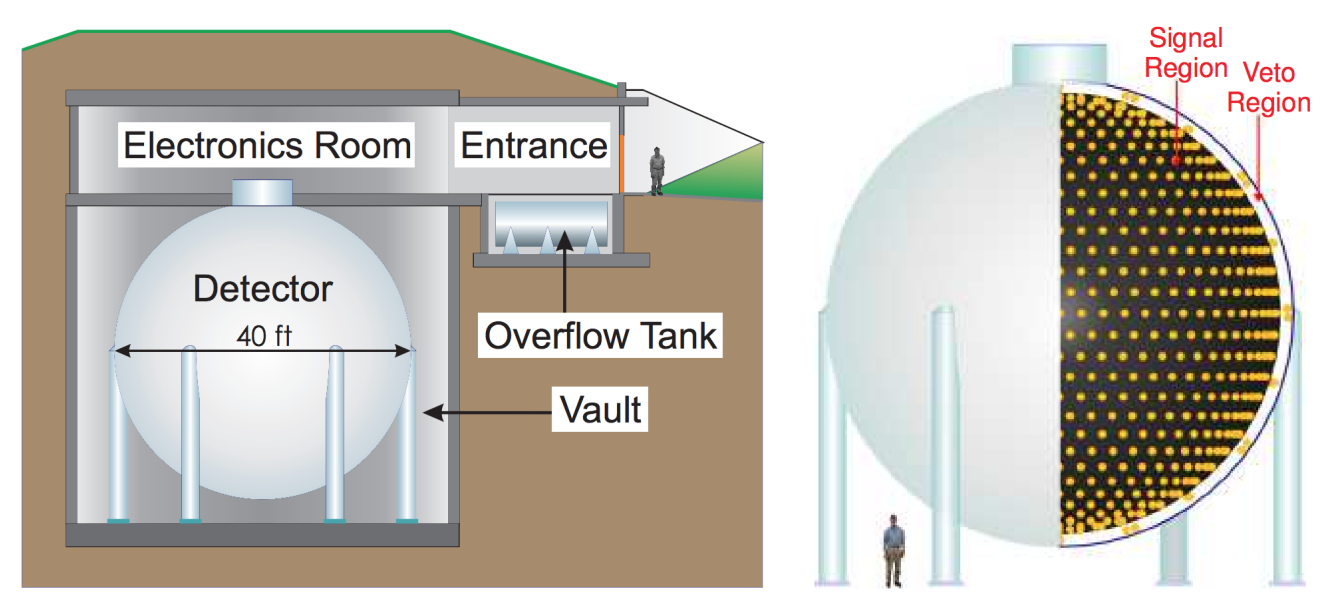

Figure B.1: Left: the MiniBooNE detector in its detector hall on the BNB under a $3 \mathrm{~m}$ earth overburden. Right: cutaway of the detector showing the signal region with 1280 inward facing PMTs and the surrounding $35 \mathrm{~cm}$ veto region containing 240 PMTs 86.

Neutrinos from the BNB are typically high enough in energy that particles produced by a neutrino-target interaction in the signal region will be above Cherenkov threshold. The Cherenkov radiation from these particles (as well as light from other sources such as late scintillation) will be observed by the signal region PMTs and reconstructed into "hits". Different types of particles will produce different signatures in the MiniBooNE detector. BNB neutrino produced muons will typically be produced at energies that make them minimum-ionizing particles in MiniBooNE 25]. As such they will typically traverse the detector without any significant deviation to their path, producing a well-defined Cherenkov ring that is observed by the PMTs. Neutrino produced electrons will repeatedly Bremsstrahlung as they travel through the detector, causing them to deviate from their path and rapidly lose energy. These two factors produce a much less well defined Cherenkov ring than that of a muon (see Figure B.2).

\section{B.2 Neutrino Events}

Every time a beam spill is sent by the BNB, MiniBooNE will receive a corresponding "beam trigger" signal and record all PMT information in a $19.2 \mu$ s time-window starting approximately $5 \mu \mathrm{s}$ before the arrival of the spill. All PMT hits produced in the beam trigger window are recorded. These hits will then be reconstructed into a "subevent": a collection of 10 or more hits with the time-difference for any 
particle track

Muon track

\section{Electron, or photon}

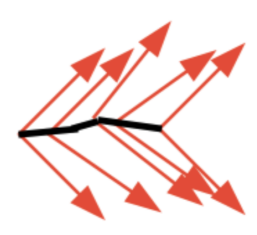

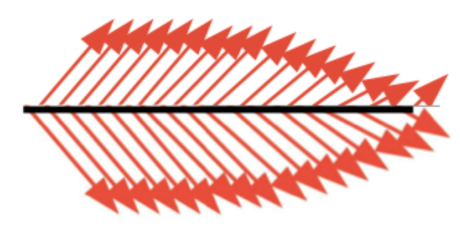

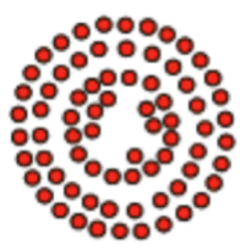

Cherenkov ring

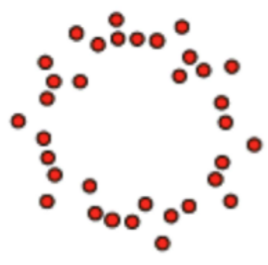

Figure B.2: Particle type and Cherenkov ring signature for muons and electrons 25].

pair of consecutive hits $<10$ ns. An event in MiniBooNE is defined as a beam trigger window that contains at least one subevent [86]. Neutrino event candidates are selected by placing two basic cosmic rejection cuts on events:

- Veto hits < 6: charged particles entering or leaving the detector will travel through the veto region, producing light (reconstructed into a hit) in the region which can be used to reject the event. Rejecting events using the veto region will reject events with cosmic particles as well as detector-external BNB neutrino events and detector-internal BNB neutrino events with charged particles that exit the signal region.

- Number of hits in first subevent $>$ 200: Michel-electrons produced by muon decay are low energy and therefore leave few hits in the detector. Such electrons are in abundance in the MiniBooNE detector due to the large number of cosmic muons. Requiring a hit upper-threshold on the first subevent will reduce the number of background Michel-electron events.

\section{B.3 Event Samples}

Four neutrino event candidate samples were used for the MiniBooNE crosschecks:

- BNB MC: Inclusive BNB neutrino-argon interactions generated using the NUANCE neutrino simulation software [70]. Used to test the selection $\nu_{\mu}$ CCQE event selection cuts used in the cross-checks in terms of the efficiency and purity of the sample. Contains 340,000 events. 
- Old data: Neutrino event candidates from the "old" MiniBooNE data set taken from 2003-2008. Contains 410,000 events corresponding to $6.4 \times 10^{20}$ POT.

- New data: The First neutrino event candidates from the "new" MiniBooNE data set taken from 2015-2016. Contains 64,000 events corresponding to $1.2 \times$ $10^{20}$ POT.

Prior to the start of new data taking, electronics were installed that were not compatible with MiniBooNE's readout. This prevented the BNB toroid data being used for POT measurements in the new data. Alternative POT accounting methods were used which were not necessarily as reliable and so there is a greater degree of uncertainty on the new data POT value.

\section{B.4 $\nu_{\mu}$ CCQE Event Selection}

Figure B.3 shows a $\nu_{\mu}$ CCQE event in MiniBooNE. A muon neutrino interacts with a neutron in a carbon nucleus to produce a proton and muon via the CCQE channel. The muon neutrino travels through the detector producing Cherenkov radiation which is reconstructed into subevent 1 . A short time later the muon decays, producing a Michel electron that leaves more Cherenkov radiation in the detector which is reconstructed into subevent 2. The proton will likely be below Cherenkov threshold and thus only leaves scintillation light in the detector. A well contained $\nu_{\mu} \mathrm{CCQE}$ event in MiniBooNE will therefore leave exactly two subevents in the detector.

For the MiniBooNE cross-checks, cuts from the MiniBooNE $\nu_{\mu}$ and $\bar{\nu}_{\mu} \mathrm{CCQE}$ cross section analyses [4] were used to isolate a $\nu_{\mu}$ CCQE sample from the data. Event selection was performed on neutrino event candidates and thus had the "veto hits $<6$ " and "number of hits in first subevent $>200$ " cuts were already applied. The $\nu_{\mu}$ CCQE selection cuts are:

- First subevent in beam-spill window: subevent time, $t$ (ns), is determined by averaging the PMT hit time of all hits in the subevent. $t$ must be within $4000<t<7000$ ns. This rejects the substantial cosmic background residing in the beam trigger window that is not in-time with the beam spill.

- Kinetic energy of the first subevent $\left(T_{\mu}\right)>200 \mathrm{MeV}$ : well contained muons will typically be above $200 \mathrm{MeV}$ in the detector. This cut rejects particles with typically lower kinetic energies such as charged pions and ensures that a muon will be well contained in the detector. 


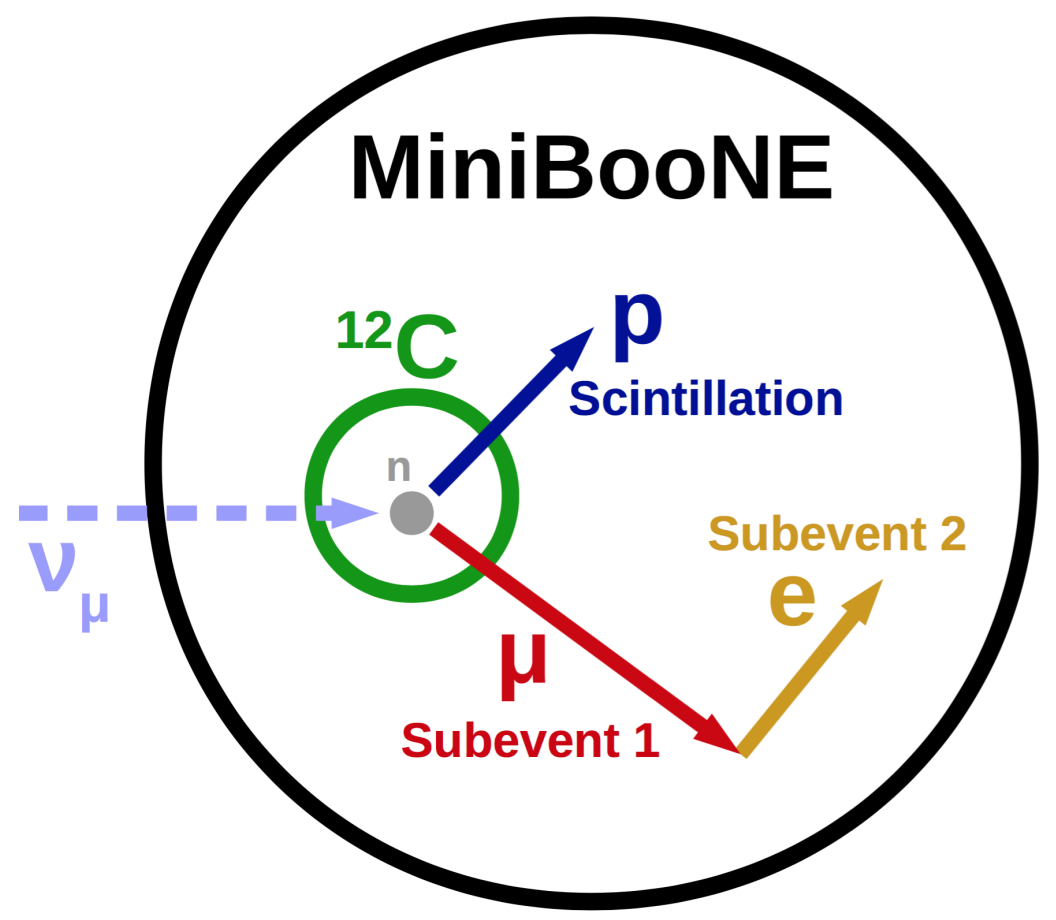

Figure B.3: A well contained $\nu_{\mu}$ CCQE event in MiniBooNE producing two subevents: one for the muon and one for the electron.

- Reconstructed subevent vertex $<500 \mathrm{~cm}$ from the center of the detector: particles on the outer edges of the detector will be very close to the signal region PMTs. This cut rejects certain types of event can be reconstructed poorly due to greater PMT sensitivity at close proximity.

- Distance between first and second subevent vertices $>100 \mathrm{~cm}$ : NC $\pi^{0} \rightarrow 2 \gamma$ decay in MiniBooNE will produce two subevents (photons) whose reconstructed vertices are close together. Muon tracks are typically long and so the muon and Michel electron vertices are typically far apart. Cutting on the 1 st and 2 nd subevent vertex distance can therefore reduce the NC $\pi^{0} \rightarrow 2 \gamma$ background with little impact on $\nu_{\mu}$ CCQE events.

- Distance between first and second subevent vertices $>500 \times T_{\mu}$ $100 \mathrm{~cm}$ : a requirement of the distance between the first and second subevent vertices to be consistent with the production and decay of a muon at the first subevent energy $T_{\mu}$.

- $\ln \left(l_{\mu} / l_{e}\right)>0$ : the likelihood of the first subevent having come from a muon $\left(l_{\mu}\right)$ must be greater than the likelihood it come from an electron $\left(l_{e}\right)$.

An additional cut requiring the number of subevents in the neutrino event candidate to be equal to two (to match the signature of a well contained muon) was part of the $\nu_{\mu}$ and $\bar{\nu}_{\mu}$ CCQE cross section analyses, however this data was not available in the 

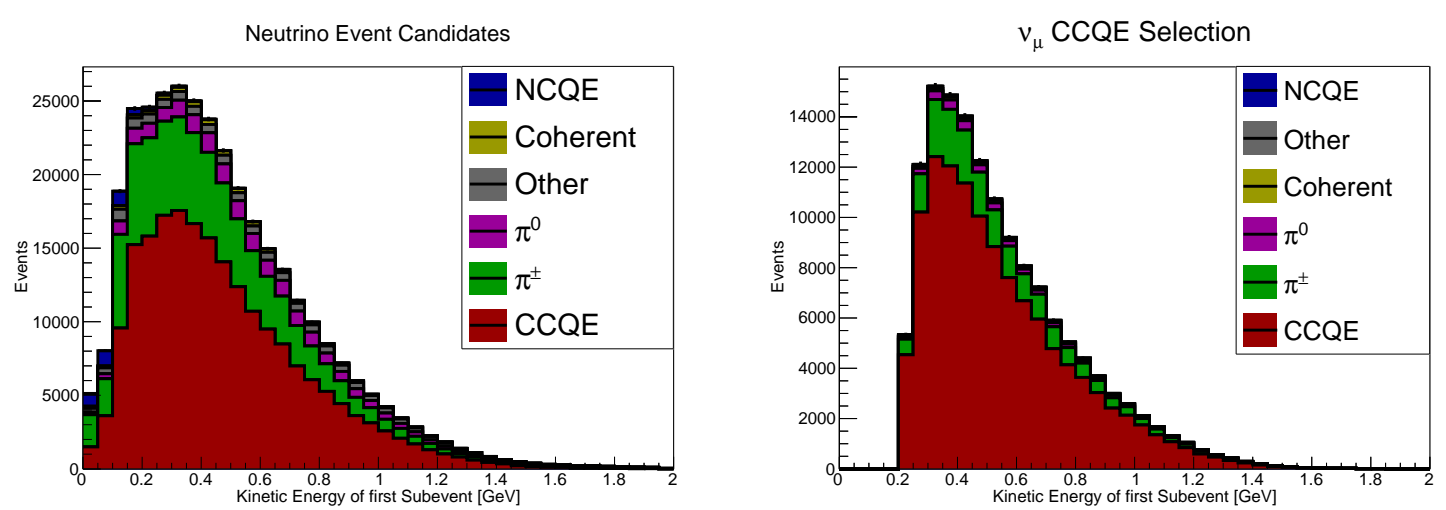

Figure B.4: Kinetic energy of the first subevent for BNB MC sample before and after selection cuts. Categorised by true neutrino interaction process.

samples used for the cross-checks and so was not applied. Figure B.4 shows the effect of applying the $\nu_{\mu}$ CCQE selection cuts on the $T_{\mu}$ variable of the BNB MC sample. The variable is broken down by the true neutrino interaction process that produced the event. The charged pion background (green) is the most substantial background to CCQE events in MiniBooNE and is significantly reduced by the selection cuts. Table B.1 lists each successive cut, the number of events in the BNB MC sample, the total efficiency and the CCQE purity of the BNB MC sample after each successive cut.

\begin{tabular}{|l|l|l|l|}
\hline Cut & Events & Efficiency \% & Purity \% \\
\hline Total & 337,000 & 100.0 & 62.0 \\
\hline First subevent in beam-spill window & 335,000 & 99.4 & 62.3 \\
\hline Kinetic energy of the first subevent $>200 \mathrm{MeV}$ & 281,000 & 83.2 & 63.8 \\
\hline Reconstructed subevent vertex $<500 \mathrm{~cm}$ from the center of the detector & 216,000 & 64.1 & 64.3 \\
\hline Distance between first and second subevent vertices $>100 \mathrm{~cm}$ & 182,000 & 54.0 & 70.0 \\
\hline Distance between first and second subevent vertices $>500 \times T_{\mu}-100 \mathrm{~cm}$ & 169,000 & 50.2 & 74.1 \\
\hline $\ln \left(l_{\mu} / l_{e}\right)>0$ & 142,000 & 42.2 & 82.0 \\
\hline
\end{tabular}

Table B.1: Number of events, efficiency and CCQE purity of BNB MC sample after each successive selection cut. Efficiency is defined as the percentage of remaining events for a given cut out of the starting total number of events. CCQE purity is defined as the percentage of remaining CCQE events out of the total remaining events for a given cut.

\section{B.5 Data Cross-check}

Figure B.5 shows the comparison between old and new data for the reconstructed neutrino vertex position in the $x, y$ and $z$ axes, the kinetic energy of the first subevent, the angle between the first subevent direction and the $z$-axis and the reconstructed neutrino energy assuming a $\nu_{\mu}$ CCQE interaction. There is a $4-5 \%$ discrepancy between the number of old and new $\nu_{\mu}$ CCQE events selected and while reasonably good shape agreement is seen between the two samples for each 
of these variables, there are some small shifts in the variable distributions. A possible cause for the difference in event rate between the old and new data could be the use of different BNB horn configurations to take the data or issues with the POT accounting performed for the new data. The downward shift in both the $T_{\mu}$ and reconstructed neutrino energy variables (middle right and bottom right plots of Figure B.5 respectively) could indicate the loss of function of some small number of PMTs in the MiniBooNE detector in the gap between old and new data taking. The loss of PMTs in the detector could also potentially explain the small shifts in reconstructed vertex position and the first subevent angle with respect to the $z$-axis.

Differences between the old and new $\nu_{\mu}$ CCQE event candidate data were studied using candidate Michel electrons calibration samples taken over the same time periods as the old and new data. Figure B.6 shows Michel electron mean energy periodically over the course of data taking for old (left plot) and new (right plot) data taking. The old data was taken in two parts: from 2003-2005 and from 2007-2008 and a decrease in mean Michel energy can be seen between these two parts. The new data was taken from 2015-2016 and shows a further decrease in mean Michel energy, showing a steady decrease in observed Michel electron energy as MiniBooNE continues to run. Comparing the total mean over the total time periods for old and new data taking shows a $1 \%$ drop for Michel energy from old to new data. A similar pattern can be seen in $T_{\mu}$ by dividing the old data into the 2003-2005 and 2007-2008 periods as shown in Figure B.7. This 1\% drop is minor and will have no significant impact on MiniBooNE's ability to take data.

Potential explanations for this decrease in reconstructed energy include a loss in functionality of approximately 13 PMTs (1\% of total) between the since the old data was taken, degradation of mineral oil purity, or changes in the kinematics of the BNB neutrinos in MiniBooNE due to the use of different horn configurations. Figure B.8 shows a comparison of the average PMT PE observed per event on each PMT channel for the old and new data. A decrease in average PMT PE is seen in the new data compared to the old which is relatively uniform across all active PMT channels. This indicates that the $1 \%$ drop in reconstructed energy is not due to a loss of PMTs. 

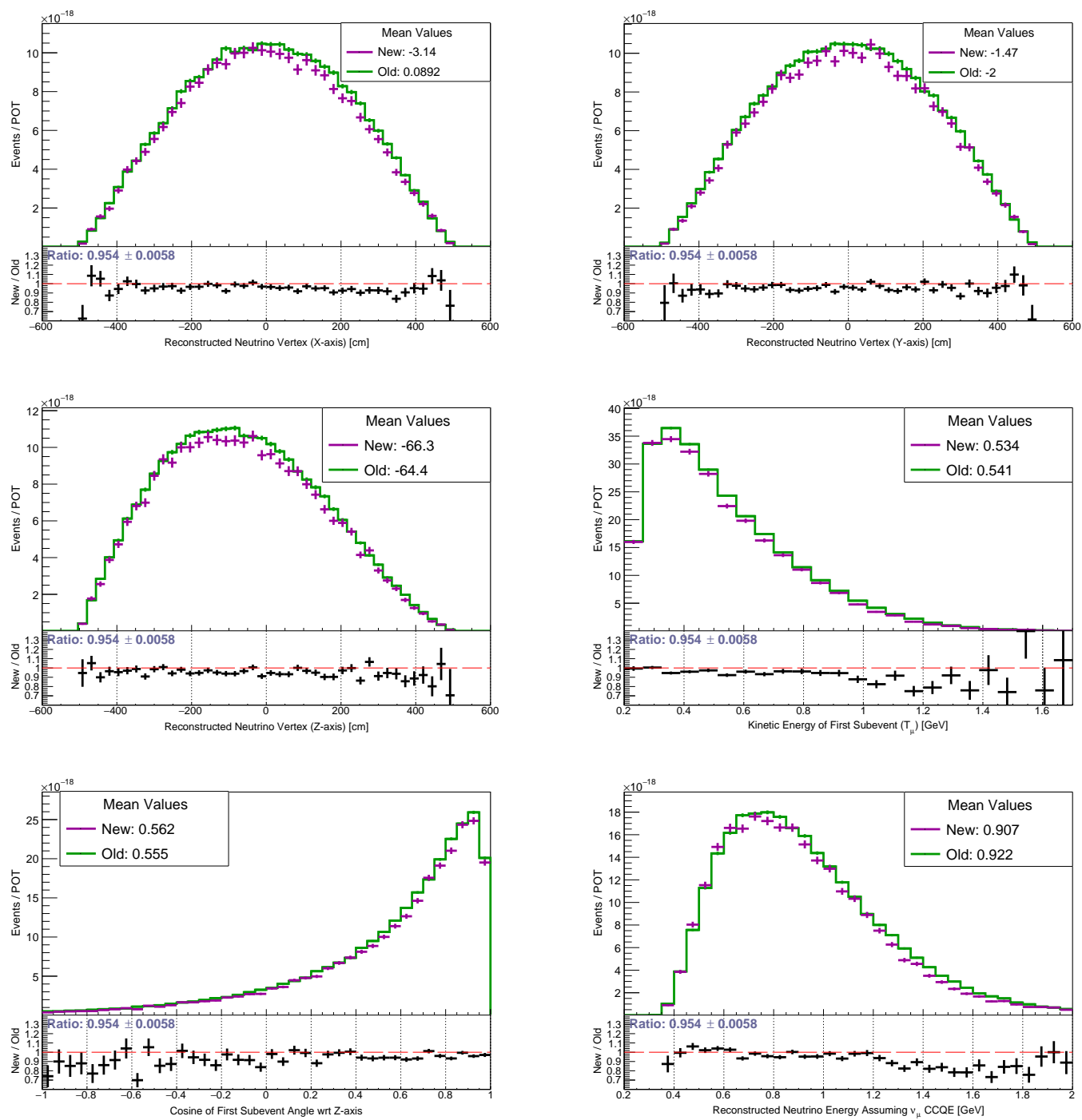

Figure B.5: Comparison between old and new data samples for several reconstructed variables post-selection. Each plot is subdivided into two panels. The top panel contains the superimposed variable distributions of the old and new data. Each sample are normalized by events/POT. The error bars on each distribution show the statistical uncertainty. Legends contain the mean for each distribution plotted. The bottom panel contains the ratio of new to old per bin, error bars show the combined statistical uncertainty for the ratio. The top left, top right and middle left plots show the reconstructed neutrino vertex position in the $\mathrm{x}, \mathrm{y}$ and $\mathrm{z}$ axes, middle right is $T_{\mu}$ (plotted from $0.2 \mathrm{GeV}$ onwards as the $>0.2 \mathrm{GeV}$ cut has already been applied), bottom left is the angle between the first subevent direction and the z-axis and bottom right is the reconstructed neutrino energy assuming a $\nu_{\mu}$ CCQE interaction. 

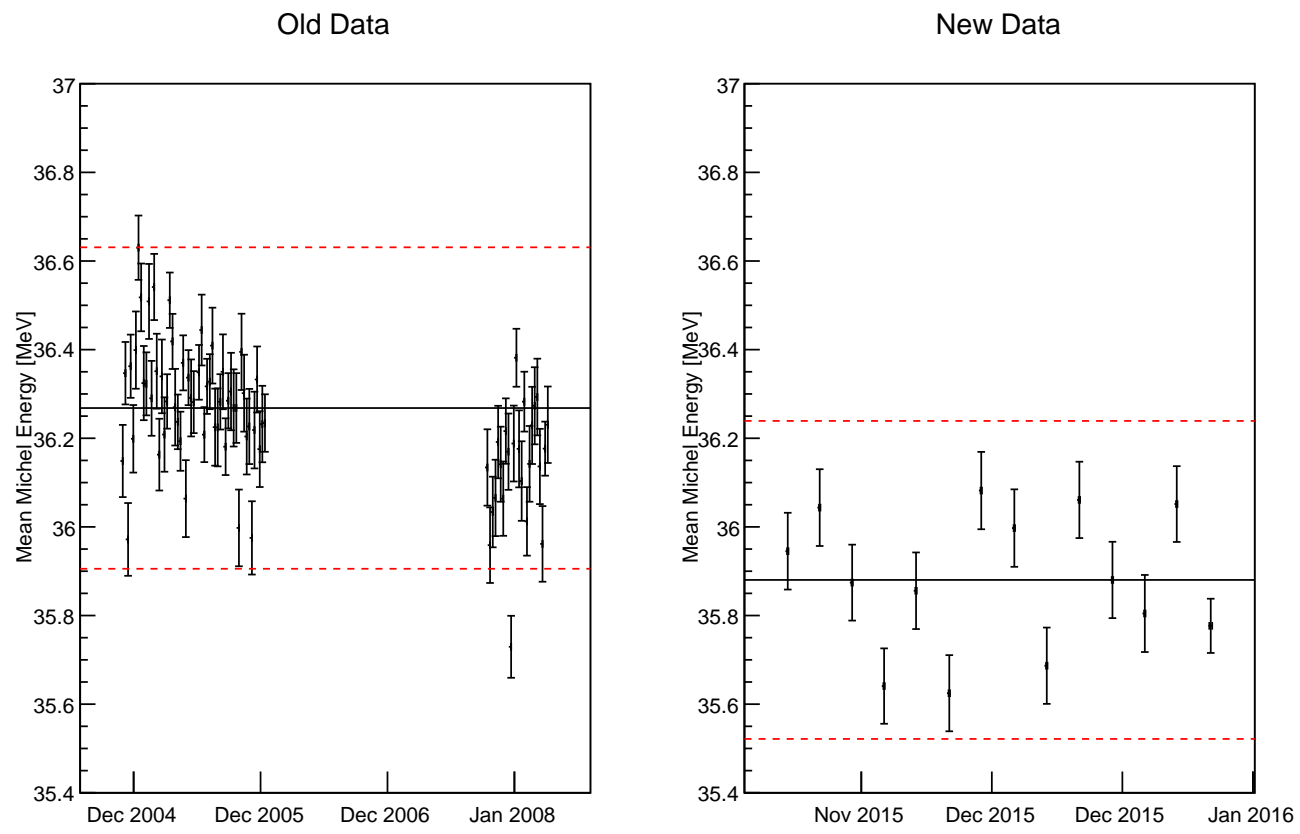

Figure B.6: Mean Michel electron energy at regular intervals over the course of data taking for the old (left plot) and new (right plot) data taking. In both plots the horizontal black line shows the total mean Michel electron energy for the entire time period in the plot. The red dashed lines show the total mean $\pm 1 \%$.

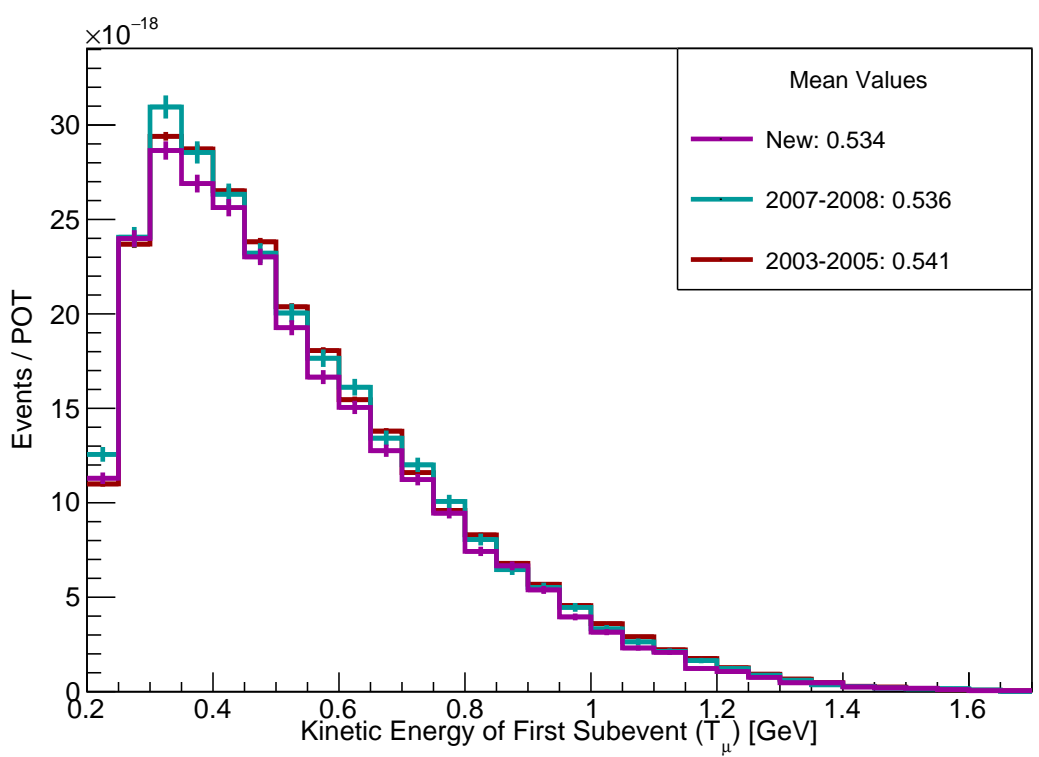

Figure B.7: Kinetic energy of the first subevent for old and new data. Old data is divided into 2003-2005 and 2007-2008 run periods. 


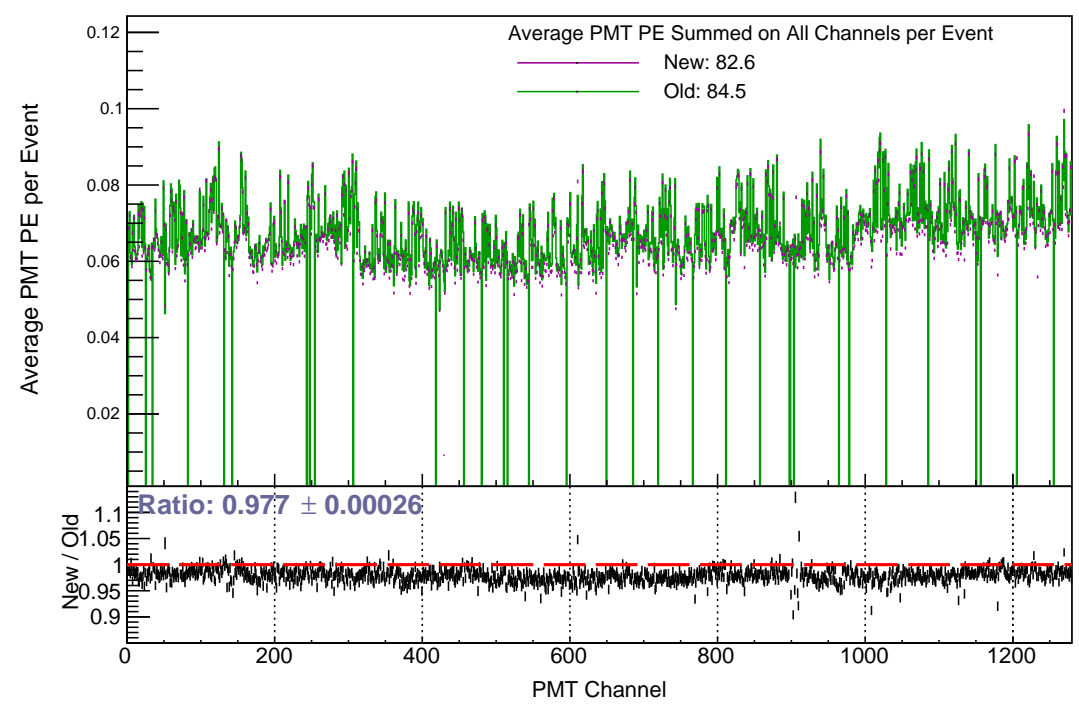

Figure B.8: A comparison of average PMT charge observed per event on each PMT channel for the old and new data.

\section{B.6 Summary}

A comparison was made between data MiniBooNE had taken in its previous run periods between 2003-2008 and the first new data taken between 2015-2016. The comparison was performed by placing a $\nu_{\mu}$ CCQE event selection on neutrino event candidates and comparing position and kinematic variables of the selected events. The new data showed relatively good shape agreement with the old data but small shifts were present in many of the variables. Studying mean Michel electron energies over the same time periods as the old and new data samples also showed a $1 \%$ decrease in Michel energy. Degradation of mineral oil purity or the use of different BNB horn configurations could account for this change. The comparison between old and new data also showed a 4-5\% lower event rate for the new data compared to the old. This could again be explained by the difference in BNB horn configurations between the two run periods or problems with POT accounting for the new data. 


\section{References}

[1] E. D. Church. LArSoft: A Software Package for Liquid Argon Time Projection Drift Chambers. arXiv:1311.6774 [physics.ins-det], 2013.

[2] Andreas Hoecker, Peter Speckmayer, Joerg Stelzer, Jan Therhaag, Eckhard von Toerne, and Helge Voss. TMVA: Toolkit for Multivariate Data Analysis. PoS, ACAT:040, 2007.

[3] Background and Resolution Studies for the MicroBooNE Search for Single Photon Low Energy Excess Search. https://www.nevis.columbia.edu/reu/ 2018/Report_Aileen_Zhai.pdf, accessed 31/12/2018.

[4] J. Grange. Extraction of $\bar{\nu}_{\mu}$ CCQE cross sections. MiniBooNE Technical Note 302, 2012. Available upon request.

[5] M. Sorel et al. A combined analysis of short-baseline neutrino experiments in the $(3+1)$ and $(3+2)$ sterile neutrino oscillation hypotheses. Phys. Rev., D70:073004, 2004.

[6] H. Päs et al. Sterile-active neutrino oscillations and shortcuts in the extra dimension. Phys. Rev., D72:095017, 2005.

[7] T. Goldman et al. Multichannel oscillations and relations between LSND, KARMEN, and MiniBooNE, with and without CP violation. Phys. Rev., D75:091301, 2007.

[8] A. E. Nelson et al. Short Baseline Neutrino Oscillations and a New Light Gauge Boson. Phys. Rev., D77:033001, 2007.

[9] E. Wang, L. Alvarez-Ruso, and J. Nieves. Single photon events from neutral current interactions at MiniBooNE. Phys. Lett., B740:16-22, 2015.

[10] J. L. Rosner. Low-energy photon production in neutrino neutral-current interactions. Phys. Rev., D91(9):093001, 2015.

[11] A. Aguilar et al. The Neutrino Flux Prediction at MiniBooNE. Phys. Rev., D79:072002, 2009.

[12] W. Haxton. The Solar Neutrino Problem. Ann. Rev. Astron. Astrophys, 33:459503, 1995. 
[13] R. Davis et al. Search for Neutrinos from the Sun. Phys. Rev. Lett., 20:1205, 1968.

[14] Q. R. Ahmad et al. Measurement of the Rate of $\nu_{e}+d \rightarrow p+p+e^{-}$Interactions Produced by ${ }^{8} B$ Solar Neutrinos at the Sudbury Neutrino Observatory. Phys. Rev. Lett., 87:071301, 2001.

[15] S. Schael et al. Precision electroweak measurements on the $Z$ resonance. Phys. Rept., 427:257-454, 2006.

[16] Y. Fukuda et al. Evidence for Oscillation of Atmospheric Neutrinos. Phys. Rev. Lett., 81:1562, 1998.

[17] Z. Maki et al. Remarks on the Unified Model of Elementary Particles. Phys. Rev. Lett., 100:100, 2008.

[18] S. Abe. Precision Measurement of Neutrino Oscillation Parameters with KamLAND. Progress of Theoretical Physics, 28:870, 1962.

[19] M. Tanabashi et al. Review of particle physics. Phys. Rev. D, 98:030001, Aug 2018.

[20] H. Ray. The MiniBooNE Experiment : An Overview. arXiv:hep-ex/0701040, 2007.

[21] A. Aguilar-Arevalo et al. Evidence for neutrino oscillations from the observation of $\bar{\nu}_{e}$ appearance in a $\bar{\nu}_{\mu}$ beam. Phys. Rev., D64:112007, 2001.

[22] A. Aguilar-Arevalo et al. Improved Search for $\bar{\nu}_{\nu} \rightarrow \bar{\nu}_{e}$ Oscillations in the MiniBooNE Experiment. Phys. Rev. Lett., 110:161801, 2013.

[23] A. Aguilar-Arevalo et al. Observation of a Significant Excess of Electron-Like Events in the MiniBooNE Short-Baseline Neutrino Experiment. arXiv:1805.12028 [hep-ex], 2018.

[24] A. A. Aguilar-Arevalo et al. First Observation of Coherent $\pi^{0}$ Production in Neutrino Nucleus Interactions with $E_{\nu}<2$ GeV. Phys. Lett., B664:41-46, 2008.

[25] G. Karagiorgi. Searches for new physics at MiniBooNE: Sterile neutrinos and mixing freedom. Ph.D. Thesis, 2010.

[26] Z. Djurcic et al. "Determination of the number of dirt events from the Booster beamline at MiniBooNE". MiniBooNE Technical Note 249, 2008. Available upon request. 
[27] C. T. Kullenberg et al. A search for single photon events in neutrino interactions. Phys. Lett., B706:268-275, 2012.

[28] P. Lasorak. A search for neutrino-induced single photons and measurement of oscillation analysis systematic errors with electron and anti-electron neutrino selections, using the off-axis near detector of the Tokai to Kamioka experiment. Ph.D. Thesis, 2018.

[29] A. Aguilar-Arevalo et al. Measurement of the Antineutrino Neutral-Current Elastic Differential Cross Section. Phys. Rev., D91:012004, 2015.

[30] MicroBooNE Collaboration. MicroBooNE low-energy excess signal prediction from unfolding. MicroBooNE Public Note 1043 http://microboone. fnal.gov/wp-content/uploads/MICROBOONE-NOTE-1043-PUB.pdf, accessed $02 / 11 / 2018$.

[31] J. M. Conard et al. Sterile Neutrino Fits to Short Baseline Neutrino Oscillation Measurements. Adv.High Energy Phys., 163897, 2013.

[32] J. Kopp et al. Sterile Neutrino Oscillations: The Global Picture. JHEP, 1305:50, 2013.

[33] K. N. Abazajian et al. Light Sterile Neutrinos: A White Paper. Fermilab Technical Publications, 2012.

[34] M. Maltoni et al. Sterile neutrino oscillations after first MiniBooNE results. Phys. Rev., D76:093005, 2007.

[35] F. Dydak. A search for $\nu_{\mu}$ oscillations in the $\Delta m^{2}$ range $0.3-90 \mathrm{eV}^{2}$. Phys. Lett. B, 134:281-286, 1984.

[36] P. Adamson et al. Search for Sterile Neutrinos Mixing with Muon Neutrinos in MINOS. Phys. Rev. Lett., 117(15):151803, 2016.

[37] P. Adamson et al. A Study of Muon Neutrino Disappearance Using the Fermilab Main Injector Neutrino Beam. Phys. Rev., D77:072002, 2008.

[38] G. Mention, M. Fechner, Th. Lasserre, Th. A. Mueller, D. Lhuillier, M. Cribier, and A. Letourneau. The Reactor Antineutrino Anomaly. Phys. Rev., D83:073006, 2011.

[39] Y. Declais et al. Search for neutrino oscillations at 15-meters, 40-meters, and 95-meters from a nuclear power reactor at Bugey. Nucl. Phys., B434:503-534, 1995. 
[40] B. Armbruster, I. M. Blair, B. A. Bodmann, N. E. Booth, G. Drexlin, J. A. Edgington, C. Eichner, K. Eitel, E. Finckh, H. Gemmeke, J. Hößl, T. Jannakos, P. Jünger, M. Kleifges, J. Kleinfeller, W. Kretschmer, R. Maschuw, C. Oehler, P. Plischke, J. Reichenbacher, C. Ruf, M. Steidl, J. Wolf, and B. Zeitnitz. Upper limits for neutrino oscillations $\bar{\nu}_{\mu} \rightarrow \bar{\nu}_{e}$ from muon decay at rest. Phys. Rev. D, 65:112001, Jun 2002 .

[41] D. Caratelli. Study of Electromagnetic Interactions in the MicroBooNE Liquid Argon Time Projection Chamber. Ph.D. Thesis, 2018.

[42] MicroBooNE Collaboration. Booster Neutrino Flux Prediction at MicroBooNE. MicroBooNE Public Note 1031 http://microboone.fnal.gov/wp-content/ uploads/MICROBOONE-NOTE-1031-PUB.pdf, accessed 02/11/2018.

[43] R. Acciarri et al. Design and Construction of the MicroBooNE Detector. JINST, 12(02):P02017, 2017.

[44] MicroBooNE Collaboration. A Measurement of the Attenuation of Drifting Electrons in the MicroBooNE LArTPC. MicroBooNE Public Note 1026 http://microboone.fnal.gov/wp-content/uploads/ MICROBOONE-NOTE-1026-PUB.pdf, accessed 02/11/2018.

[45] B. Baller. Liquid Argon TPC Signal Formation, Signal Processing and Hit Reconstruction. JINST, 12(07):P07010, 2017.

[46] R. Acciarri et al. Michel Electron Reconstruction Using Cosmic-Ray Data from the MicroBooNE LArTPC. JINST, 12(09):P09014, 2017.

[47] M. E. Shibamura et al. Drift velocities of electrons, saturation characteristics of ionization and $\mathrm{W}$-values for conversion electrons in liquid argon, liquid argongas mixtures and liquid xenon. Nucl. Instrumentation Meth, 131, 1975.

[48] M. Miyajima et al. Average Energy Expended per Ion Pair in Liquid Argon, Liquid Argon-gas Mixtures and Liquid Xenon. Phys. Rev, A9:1438, 1974.

[49] V. Meddage et al. "Detector Calibration using stopping and through going muons". https://microboone-docdb.fnal.gov/cgi-bin/private/ RetrieveFile?docid=14754, accessed 18/11/2018, 2018. Available upon request.

[50] M. J. Berger et al. Estar, pstar, and astar: Computer programs for calculating stopping-power and range tables for electrons, protons, and helium ions (version 1.2.3). https://physics.nist.gov/PhysRefData/Star/Text/ESTAR.html, accessed 15/09/2018, 2005. 
[51] E. Segre. Nuclei and particles: An introduction to nuclear and subnuclear physics. W.A. Benjamin, 1964.

[52] Y. S. Tsai. Pair production and bremsstrahlung of charged leptons. Rev. Mod. Phys., 46:815, 1974.

[53] M. Antonello et al. A Proposal for a Three Detector Short-Baseline Neutrino Oscillation Program in the Fermilab Booster Neutrino Beam. arXiv:1503.01520 [physics.ins-det], 2015.

[54] MicroBooNE Collaboration. First Muon-Neutrino Charged-Current Inclusive Differential Cross Section Measurement for MicroBooNE Run 1 Data. MicroBooNE Public Note 1045 http://microboone.fnal.gov/wp-content/ uploads/MICROBOONE-NOTE-1045-PUB.pdf, accessed 02/11/2018.

[55] MicroBooNE Collaboration. First Measurement of Muon Neutrino Charged Current Single Neutral Pion Production on Argon with the MicroBooNE LArTPC. MicroBooNE Public Note 1045 http://microboone. fnal.gov/wp-content/uploads/MICROBOONE-NOTE-1032-PUB.pdf, accessed $28 / 12 / 2018$.

[56] C. Andreopoulos et al. The GENIE Neutrino Monte Carlo Generator: Physics and User Manual. arXiv:1510.05494 [hep-ph], 2015.

[57] S. Agostinelli et al. GEANT4: A Simulation toolkit. Nucl. Instrum. Meth., A506:250-303, 2003.

[58] A. Bodek and J. L. Ritchie. Further Studies of Fermi Motion Effects in Lepton Scattering from Nuclear Targets. Phys. Rev., D24:1400, 1981.

[59] C. H. Llewellyn Smith. Neutrino Reactions at Accelerator Energies. Phys. Rept., 3:261-379, 1972.

[60] L. A. Ahrens et al. Measurement of Neutrino - Proton and anti-neutrino Proton Elastic Scattering. Phys. Rev., D35:785, 1987.

[61] D. Rein et al. Neutrino Excitation of Baryon Resonances and Single Pion Production. Annals Phys., 133:79-153, 1981.

[62] R. P. Feynman, M. Kislinger, and F. Ravndal. Current matrix elements from a relativistic quark model. Phys. Rev., D3:2706-2732, 1971.

[63] A Bodek and U. K. Yang. Higher twist, xi(omega) scaling, and effective LO PDFs for lepton scattering in the few GeV region. J. Phys., G29:1899-1906, 2003. 
[64] D. Yu. Bardin et al. Muon energy spectrum in inverse $\mu$-decay. Nucl. Phys., B287:839-851, 1987.

[65] William J. Marciano and Zohreh Parsa. Neutrino electron scattering theory. J. Phys., G29:2629-2645, 2003.

[66] Z. Koba, Holger Bech Nielsen, and P. Olesen. Scaling of multiplicity distributions in high-energy hadron collisions. Nucl. Phys., B40:317-334, 1972.

[67] Torbjorn Sjostrand, Stephen Mrenna, and Peter Z. Skands. PYTHIA 6.4 Physics and Manual. JHEP, 05:026, 2006.

[68] D. Heck, J. Knapp, J. N. Capdevielle, G. Schatz, and T. Thouw. CORSIKA: a Monte Carlo code to simulate extensive air showers. Tech. Rep., FZKA 6019, 1998.

[69] Yoshinari Hayato. A neutrino interaction simulation program library NEUT. Acta Phys. Polon., B40:2477-2489, 2009.

[70] D. Casper. The Nuance neutrino physics simulation, and the future. Nucl. Phys. Proc. Suppl., 112:161-170, 2002.

[71] E. Wang, L. Alvarez-Ruso, Y. Hayato, K. Mahn, and J. Nieves. Photon emission in neutral current interactions at the T2K experiment. Phys. Rev., D92(5):053005, 2015.

[72] Richard J. Hill. Low energy analysis of $\nu n \rightarrow \nu n \gamma$ in the standard model. Phys. Rev. D, 81:013008, Jan 2010.

[73] G. T. Garvey et al. Recent Advances and Open Questions in Neutrino-induced Quasi-elastic Scattering and Single Photon Production. Phys. Rept., 580:1-45, 2015.

[74] E. Wang, L. Alvarez-Ruso, and J. Nieves. Photon emission in neutral current interactions at intermediate energies. Phys. Rev., C89(1):015503, 2014.

[75] Xilin Zhang and Brian D. Serot. Can neutrino-induced photon production explain the low energy excess in MiniBooNE? Phys. Lett., B719:409-414, 2013.

[76] G. T. Garvey. "Photon Decay of the Delta". MiniBooNE Technical Note 192, 2008. Available upon request.

[77] J. S. Marshall and M. A. Thomson. The Pandora Software Development Kit for Pattern Recognition. Eur. Phys. J., C75(9):439, 2015. 
[78] MicroBooNE Collaboration. The MicroBooNE Search for Single Photon Events. MicroBooNE Public Note 1041 http://microboone.fnal.gov/wp-content/ uploads/MICROBOONE-NOTE-1041-PUB.pdf, accessed 02/11/2018.

[79] R. Acciarri et al. First Observation of Low Energy Electron Neutrinos in a Liquid Argon Time Projection Chamber. Phys. Rev., D95:072005, 2017.

[80] M. Mooney. "Misconfigured/Dead Channels for Run 1". https: //microboone-docdb.fnal.gov/cgi-bin/private/RetrieveFile?docid= 7345, accessed 18/12/2018, 2017. Available upon request.

[81] Byron P. Roe, Hai-Jun Yang, Ji Zhu, Yong Liu, Ion Stancu, and Gordon McGregor. Boosted decision trees, an alternative to artificial neural networks. Nucl. Instrum. Meth., A543(2-3):577-584, 2005.

[82] R. Brun et al. ROOT - An Object Oriented Data Analysis Framework. Nucl. Instrum. Meth., A389, 1997.

[83] R. Acciarri et al. A study of electron recombination using highly ionizing particles in the ArgoNeuT Liquid Argon TPC. JINST, 8:P08005, 2013.

[84] A. Schukraft. "Normalization of data and MC events". https: //microboone-docdb.fnal.gov/cgi-bin/private/RetrieveFile?docid= 5640, accessed 15/12/2018, 2016. Available upon request.

[85] C. Adams et al. A Deep Neural Network for Pixel-Level Electromagnetic Particle Identification in the MicroBooNE Liquid Argon Time Projection Chamber. Fermilab Technical Publications, 2018.

[86] MiniBooNE Collaboration. The MiniBooNE Detector. Nucl. Instrum. Meth., A599:28, 2009.

[87] R. G. Van der Water. "Low Energy Dirt Optimization Cuts". MiniBooNE Technical Note 251, 2008. Available upon request.

[88] K. Duffy et al. "Particle Identification in the MicroBooNE Time Projection Chamber". https://microboone-docdb.fnal.gov/cgi-bin/private/ RetrieveFile?docid=16175, accessed 14/12/2019, 2018. Available upon request.

[89] M. Ross-Lonergan. "Appendix files for SinglePhoton collaboration Meeting: data-mc and training variables". https://microboone-docdb.fnal.gov/ cgi-bin/private/RetrieveFile?docid=20788, accessed 26/02/2019, 2019. Available upon request. 
[90] Alexander L. Read. Presentation of search results: The CL(s) technique. J. Phys., G28:2693-2704, 2002. 